


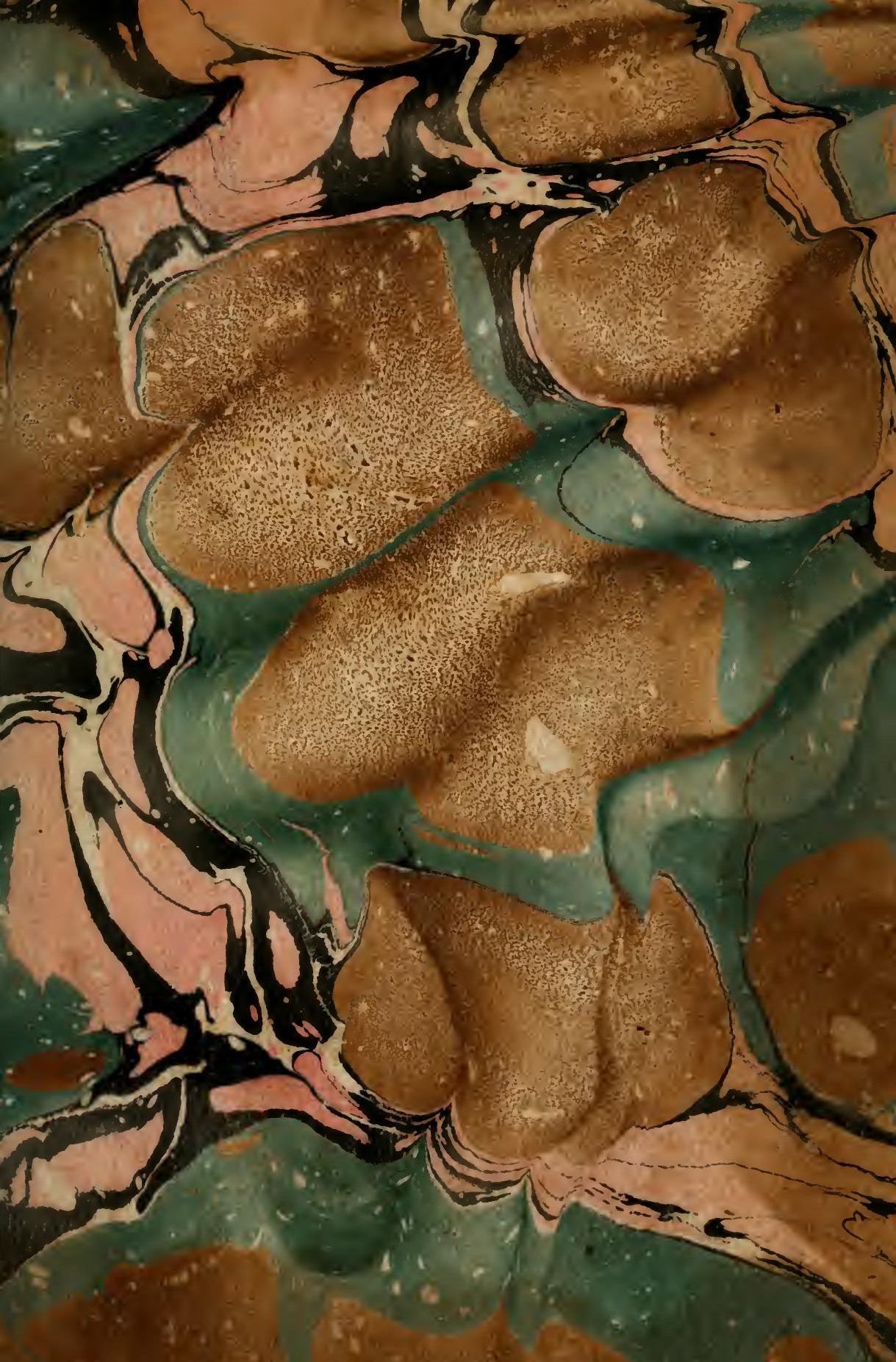




$$
\text { . }
$$

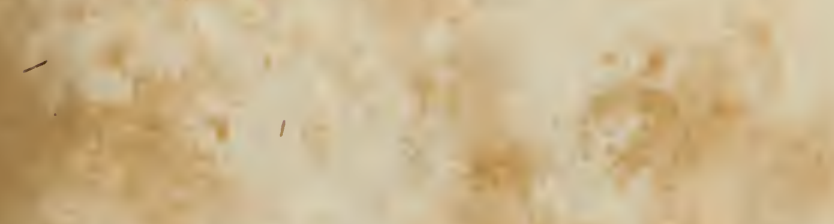

$$
\begin{aligned}
& \text {. }
\end{aligned}
$$

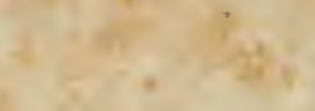


CA

\section{BOTANICORUM PRINCIPIS,}

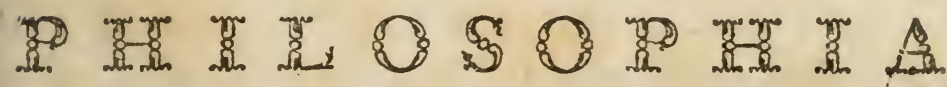

$$
\text { B O T A N I C A, }
$$

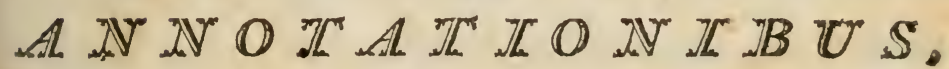
EXPLANATIONIBUS, SUPPLEMENTIS

$$
A U C T A
$$

$$
\text { CURA", ET OPERÄ }
$$

CASIMIRI GOMEZ ORTEGA, Med. D. et Botan. in Hort.Reg. Matr. Professoris A C C E D U N T

F. Andr. Murray Nomina trivialia.

\section{MATR ITI:}

EX TYPOGR. VIDUA, ET FILII PETRI MARIN. ANNOM.DCC.XCII. 

CASIM. GOMEZ ORTEGA

\section{PR Æ F A T IO.}

Caroli Linnæi, Botanicorum Principis, Pbilosophiam Botanicam, Opus eximium, tyronibus necessarium, in arte provectis haud inutile, Institutionum omnium, quotquot hactenus prodierunt, fundamentum, optimorum Professorum suffragiis approbatum, et certatim apud exteras Nationes typis propriis evulgatum, in Hispaniæ Bibliopoliis penitus desiderari, aut ejus exemplaria ægrè inveniri, et immodico pretio venundari, vehementer dolebam.

Super hæc Botanicen publicè docendi munus, quo præter meritum ampliùs viginti abhinc annis fungor, nimium frequentes opportunitates mihi obtulerat intelligendi, plura in hoc aureo Libro auditores offendere, quæ eis difficultatem haud exiguam facesserent. Nimirum immortalis Auctor singulari, qua erat, mentis acie Lectorum 
ingenia, qui præstantissimorum quorumque hominum mos est, ponderabat, et aphoristicæ brevitatis impensè studiosus perspicuitati, et tyronum captui minùs, quàm oportuisset, quandoque inserviebat. Quamobrem Professorii muneris partes esse, atque adeo operæ pretium me facturum putavi, si Linnæi doctrinam, eorum instar, qui classicos Auctores interpretantur, commentariis, atque annotationibus explanarem.

Repetitâ Editionis curâ, quam pene jam jam absolutam per binos annos propter alia negotia, et infirmam valetudinem intermittere cuactus fueram, videre mihi contigit Linnei Pbilosopbiam auctam, et emendatam curâ Car. Lud. Wildenow, $M$. D. Berol. 8. 1790 , in qua nonnulla reformata, alia mutata, plura addita ex recentiorum detectis, præsertim diligentissimorum Botanicorum Gaetner, et Hedwigii in plantis cryptogamicis Lectoribus utilitatem cum jucunditate parient. At doctissimi Viri, mihique amicissimi, Dominici $\mathrm{C} i$ - 
rilli Fundamenta Botanica, sive Philosopbice explicationem, Neapoli nuper editam, legendi desiderio adhuc teneor.

Editionem verò clar. Wildenow pervolvens, aliam omnino viam me institisse, cognovi, prout illa cum meis conferenti patebit: neque enim tam mihi proposui, Linnæi doctri nam novis inventis amplificare, aut textum immutare, nullâ præcipuè ratione adhibitâ, quod Wildenow ipse brevitatis causâ se fecisse, et audacter factum videri sibi pcr suasum esse in Præfatione fatetur, quàm lo. ca tyronibus obscuriora, et difficilia explana. re. Quoties tamen me coegit necessitas, auc. toritati veritatem liberè prætuli; sed id extra textum feci, quem invertere, aut intercipere religioni pene habui, et in Annotatio. nibus innovationum fundamentis ubique expositis doctiorum judicio rem integram reservavi.

Menda typographica, aut etiam Grammatices vitia, quibus Holmiensis, et ceteræ Editiones scatebant, quæque in hac nos- 
trâ corrigere studuimus, enumerare supersedemus, ne videamur laureolam in mustaceo quærere voluisse.

Appendicis autem, seu Philosophiæ Botanices Supplementi loco huic editioni adjicienda curavi ill. F. Andr. Murray Nomina trivialia, quod ejusdem Philosophiæ Caput, in Linnæano Opere inter Cap. IX. $V$ arietates, et Cap. X. Synonima interponi dignum, desiderabatur.

Ad extremum, quoniam Linnæus quotiescumque plantas in hac Philosophiâ memorat, nominibus tantùm Genericis, aut etiam Specificis usus est, quæ deinceps in $S p e c$. Plant. multò posteriùs editis reformavit; aliquantulum me à Tyronibus gratiæ initurum putavi, si Indicem Nominum plantarum Philosopbice Botan. ad earumdem Nomina in Specierum Opere reperienda reductorum subjicerem. 


\section{LECTORI BOTANICO \\ CAR. LIN N EUS.}

undamentis Botanicis Theoriam atque Institutiones Rei Herbariæ sub paucis aphorismis olim comprehendi, quorum explicationem per exempla, observationes, et demonstrationes, distinctis, riteque definitis plantarum partibus, et terminorum vocibus, Pbilosopbiam Botanicam dixi, cùm in his consistant præcepta Artis.

Philosophiæ ejusmodi Botanicæ varias partes dudum emisimus utpote in Cap. I. Bibliothecam Botanicam; II. Classes Planw tarum; V. Sponsalia Plantarum; VII. VIII. IX. X. Critican Botanicam; XII. Vires Plantarum; reliquas Sectiones fundamen* torum, sc. Cap. III. IV. VI. XI. conjunctim cum prioribus in unum opus compingere, et auctas novis exemplis, observationibus, 
demonstrationibus sub Philosophiæ Botanicæ titulo edere, diu animo volvi, in quem etiam finem bene multa collegi; inte rea territum copiâ dicendorum satietas, imó tædium operosæ scriptionis me cepit, adeo ut hæc in tempora commodiora seponere visum fuerat, quæ cum annis, auctis indies curis publicis et privatis, muneris negotiis, et peregrinationibus Historiæ Naturalis causâ institutis, ita sese subtrahebant, ut de succesu operis ipse dubitare cœperim.

Interea urgente Bibliopolâ, post distracta priora exemplaria, novam Fundamentorum Botanicorum editionem à me quoque enixè petiere discipuli, ut $P$ artes plantarum, artisque terminos, à me usitatos, rite definitos adderem, methodo, qua eos in prælectionibus tradere solitus sum; huic eorum petitioni accessere amicorum, de re Herbaria optimè meritorum, adhortationes, ut terminos artis explicarem, partesque plantarum definirem. 
Ut amborum desideriis adsurgerem, incepi collectanea mea in compendium redigere, et typis mandare: at dira arthritis, vix incepto opere, ita una cum corporis viribus mentem, et animum fregit, ut in ipsâ herbâ ferè suffocatum fuisset. Nunc veró, post recuperatas qualitercumque vires, Philosophiæ Botanicæ compendium heic sisto, mole parvum, utpote primas lineas, primaque rudimenta Botanices, in gratiam tantùm discipulorum editum, complectens, sed quod alio tempore, si fata velint, et otia, longe auctius prodibit.

In calce Opusculi adjeci nonnulla de instructione disciplinæ, ne paginæ alternæ vacuæ relinquerentur.

Occupatus nunc in colligendis Speciebus Plantarum, præstantissimos per Europam Botanicos enixè rogo, oroque, ut plantas flore instructas, si quas possideant in duplo rariores, vel à me non dictas, mecum communicent, quo liceat 
easdem ad Genera referre cum adæquatis Differentiis: meum erit vicissim in Opere, ad quamcum que speciem, publicè testari gratum animum in eos, qui easdem com. municarunt.

\section{Upsalice I750. Septembr. 16.}

Pascitur in vivis livor, post fat a quiescit; Tunc suus ex merito quemque tuetur bonos. 


\section{B I B L I O T H E C A.}

5. BIBLIOTHECA Botanica continet Libros de Vegetabilibus scriptos.

Docet Detecta, Fata, Progressus, Loca, Methodum.

Sciat Botanicus, quos evolvat auctores de planta qurstionis. Europæa. C. Baubinus (cum systematico), Ravjus, 3. Vaillant. Alpina. Rajus, Linnæus, Hallerus, Gmelinus.

Capensia. Hermannus, Burmannus, Commelinus, Linnæus, Rajus, Plukenetius, Petiverus.

Indica. Hermannus, Rbeede, Plukenetius, Rajús, Bürmannis, Linneus.

Americ. septentr. Gronovius, Catesbous, Rajus, Morisonus, Plukenatius, Cornutus.

.. .. .. austral. Plumierus, Sloaneus, Rajus, Plukenetius, Hernandez, Marcgravius, Piso, Feuilleus.

Bibliothécæ. Seguierus, Linneus, Bumaldus, Scheuchzerus. Historie: Tournefortius, Hotto, Royenus.

6. PHYTOLOGI vocantur Auct res opere aliquo (5) de vegetalibus clari, sive Botanici, sive Botanophili sint.

Fund. Bot. \$. 7. cum 43. distinctio.

Botanici primarii secundum Tempus.

Theophrastus. XVII. SECULO.

Plinius.

Dioscorides.

$X V$. SECULO.

Gaza.

Barbarus.
Robinus

Spigelius

Boot

Renealme

Swertius

Biy

Beflerus

Jungermannus

Brosscus

Parkinson

Donatis

Laurembergius

Ferrarius

Cornutus

Verlingius

Stapelius I 601 1608 3609 $16 \mathrm{II}$ $16 \mathrm{I} 2$ i $\ddot{6}$ is 1613 I 628 1629 1631 1632 I 333 1635 1636 1644
XVIÍl. SECULO.

Sherard

Rudbeck $f$.

Meriana Jussiæus A. Boerhaave Petitus

Heucherus

Kempferus

I 701

1705

1709

1710

1711

1712

Helving

Tita

Feuillée

1713

1714

1716 Knaut f. Bradlaus

Isnard

VAILLANT

1717

1718

XVI. 
XVI. SECULO.

Brunfelsius

Tragus

Cordus

Ruellius

Dorstenius

GESNERUS

Fuchsius

Brassavola

Matthiolus

Lonicerus

Dodonxus

Bellonius

Guilan linus

Anguillara

Calceolarius

Pena

Lobel

Garcias.

Monardes

Clusius

Carrichter

Costreus

Acosta

C $₫ S A L P I N U S$

Durante

Dalechampius

Camerarius

Tabernæmontanu

Thalius

Alpinus P.

Bauhinus J.

Cortusus

Columna
Hernandez I 647 Pontedera 17 I 8 Marcgravius 1648 Ruppius

Piso

Turnerus i65 1 Montius

1719 Pancovius 1654 Buxbaum i 721

Loeselius

Jungius

Ambrosinus

1530 foncquet

I 532 Kudbeck

I 535 Rajus

I 536 Hoffinan $M$

I 540 Schasffer

I 54 I Elsholtz

1542 Vallot

I 545 Ctabraus

I 54 X MTret

I 550 Bo:co

.... Tillius

1723

1657 Jussixus B. 1725

.... Martyn 1726

1658 Michelius 1729 1659 Catesb.rus I73 I 1660 Geoffroy .... 1662 Celsius 1735

.... Waltherus .... i 663 Zannichellius.... It665 Linnzus .... I666 Hallerus .... I667 Gleditsch-1736 I668 Miller

1552 Aldrovandus .... Burmannus 1737

I 53 MORISON i669 Ludwig .... I 557 Munting. I672 Mochring .... I56 i Barrelier † 1673 Weinmann .... I506 Tillands $\quad \ldots$ Blackwel 1739 I 570 Sterbeck 1675 Amman f. .... .... Zannonus .... Gronovius .... Is74 Ammannus .... Royen $174^{\circ}$ .... Dodart $\quad 1676$ Scguier $\quad .$. . I576 Breynius $\quad 1678$ Barrere 174I .... Rheede $\quad \ldots$ Gerherus 1743

1578 Mentzelius 1682 Gesnerus +1744 .... Commelinus 1683 Stellerus +1746 1583 Triumfettus 1685 Gmelinus 1747 I584 Magnol 1696 Guettard .... 1587 Hermannus 1687 Wachendorff.. .... Rivinus I690 Lecheus $174^{8}$ 588 Plukenetius i69 I Dalibard 1749 .... Petiverus 1692 Kalmius 1750 159 I Cupanus ..... Hasselquist 1750 .... Plumier $\quad 1693$ Schiera 1750 .... TOURnefort I69+ SOCIETAS. 1592 Bromel Germanica 1670 Anglica 1682 A 2 BAU- 
BAUHINUS.C. I 593 Zuingerus " 6 I6 Gallica I 699

Pona. "I595 Sloane .... Upsaliensis 1720

Gerardus

I 597 CommelinC. I 697 Ruthenica

1728

Imperatus

I 599

Bobartus

I 699

Noribergensis 1731

Sruenckfeld

I 600 Vo!kamerus 1700 Holmensis

1739

7 BOTANICI (veri 6) ex fundamento genuino Botanicam (4) intelligunt, et vegetabilia (2) omnia nomine intelligibili nominare sciunt; sunt hi vel Collectores (8), vel Metbodici ( 18 ).

Fund. Bot. I51. 164.165.166. 167. 152.

Syst. nat. 6. p. 2 I I. \$. I 3.I2. I I. et p. 2 I5. p. 2 .

Gen. plant. pref. \$. 1. 2. 3.

Class. plant. prefat. \$. I.

8. COLLECTORES (7) de numero specierum Vegetabilium primario solliciti fuere: sunt hi Patres (9), Commentatores (Io), Ichniografbi (I I), Descriptores( I 2), Monographi (1 3), Curiosi (s 4), Adomides (I 5), Florista (I6), Peregrinatores (I 7 ).

9. PATRES (8) Botanices prima rudimenta deposuerunt.

Fata: Greci ab Agyptiis, hi a Chaliteis.

Romani devicto Pompejo ad Actium.

Gotbi sec. IV. Longabardi sec. V. Romam devastant. Arabes sec. VI. Egyptum, sec. VII. Hispaniam intrant. Marocce sec. XI. Rex Calipha.

GrIECI ante Christum natum floruere.

Hippocrates Parens Medicinæ, secul. V. ante Chr. Aristoteles Princeps Peripatheticorum, sec. IV.

Theophrastus Parens Botanices, sec. III.

Xenophon Atheniensis, sec. II.

Romani primis seculis æræ Christianæ.

Cato sub Julio Casare, An. ante Chr. I 49.

Varro sub Augusto, An. ante Chr. 62.

Virgilius sub Augusto, An. ante Chr. 70.

Columella sub Claudio.

Plinius sub Tiberio ad Titum.

Dioscorides sub Nerone.

Rufus sub Trajano.

Palladius sub Antonino Pio. 
ASFATTCF:

Galenus Pergamensis vixit Romæ An. I33.

Oribasius Pergamensis Quæstor Juliani.

Etius Amidenus, sub Constantino, et Theodosio.

AEgineta ( Paulus) sub Constantino pogonata.

ARABES:

Mesue, Nisaburensis, seculi VIII. fine.

Serapio filius Fobannis, Medicus.

Razis natus Raj Persire, circa 920

Avicenna e Boccharet Persix, Medicus, circa 1030

Averrboës e Corduba Hispania, circa sec.XI. Avenzoar

Alenguefit.

Medicus, Arabus, seculó XII.

OBSCURI seculo XII. cum insequentibus proximis, ut Myrepsius Præpositus, Quiricius Tertonensis, Hildegardis Abadissa, Sylvaticus, Dondis, Suardus, Villanova, Cuba. Platearius \&c.

Adumbrationes 325. 326. 332. ignorabant omnes.

Græcè:

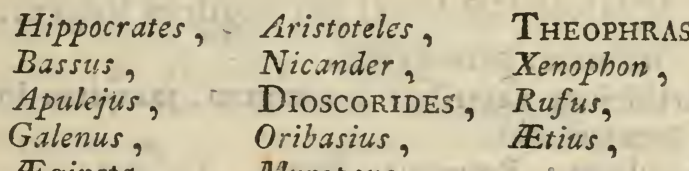

Latinè: Agineta.

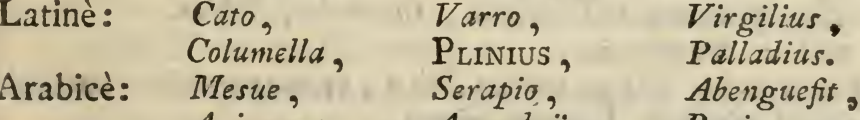

Barbarè: Cuba, Hildegardis, Myrepsus, Avicenna, Averrboës, Rasis,

Medica: Hippocrates, Galenus.

Naturalia: Aristoteles, Plinius.

Botanica: Theophrastus, Dioscorides, Plinicis.

\section{$\mathrm{A}_{3} \mathrm{COM}-$}


ı. COMMENTATORES (8) Patrum (9) scripta dilucidarunt.

Fata seculi XV. medio: Turca separavit imperium Græcum s. orientale ab occidentali; aufugxe in Italiam Litteras adduxere ; inventa: Typographia 1440. Pulvis pyrius. Anerica 1492.

ARISTOTELEM transtulit Constantinus \&c. commentaria dedit Scaliger \&c.

THEOPHRASTUM transtul. Gaza, \&ic.

comment. Stapel, Scaliger, \&c.

PLIN. castigarunt Dalechampius, Harduinus, Gronovius, \&c. comment. Barbarus, Guilandinus, Salmasius, \&c.

DIOSCORIDEM transtul. Cornarus, Sarracenus, \&c. comment. Barbarus, Fucbsius, Matthiolus, Cordus, Gesnerus, Ruellius, \&c.

CATONEM comment. Meursius, Ursinus, \&c.

A VICENNAM transtul. Alpagus, Costreus, Plempius, \&c. Amalthous hebraicè.

comment. Lonicerus.

MESUEM comment. Campegius, Monardus, \&c.

I I. ICHNIOGRAPHI (8) Figuras Vegetabilium Iconibus expresserunt.

Artificium veteribus inusitatum, tanquam in speculo repræsentatio.

Requisita: Botanicus, Pictor, Sculptor.

hinc præstant: Dillenius, Columna, Ebret.

malè : Muntingius.

Observanda partes onnes, situ, et magnitudine naturali, etiam minimæ Fructificationis.

Sunt vel LIGNLAE, in quibus excelluit Gesnerus, Rudbeckius. ANEA Dondis i5 36, Ferrarius, Dodartius, Breynius, Commelinus, Laselius, Rhedeus, Hermannus, Rivinus.

STANNEAt: Dillenius.

FUNDAMUNTALES absque umbra: ut Brunfelsius, Fuchsius, Clusius, Plumierus.

FUCAT球 vivis coloribưs: Martynus: Blackwell, Weinmann.

ORIGINALES ex foliis ipsis toco typi. Hesselius in America 1707, Knipphofus in Germania 1733. PRETIOSE: Rbeede, Slowne, Dillenius. 
MALAE: Brauner, MYyller, Brunszuig, Cuba, Lonicerus, Nylandus, Palmherg, Hernandez.

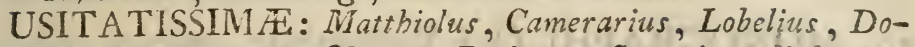
donsus, Fuchsius, Clusius, Beslerus, Swertius, Rolertus, Morisonus, Rivinus, Vaillantius.

EXOTICIS: Morisonus, Plukenetius, Petiverus, Dodartus, Martynus, Alpinus, B.trrelicrus, Bo conus, Catesbieus, Dillenius, Hermannus.

HERBARIUM præstat omni Icone, necessarium omni Botanico.

1. Plantee non humidæ colligendre.

2. Partes nullæ auferendæ.

3. moderatè explicande.

4. non verò infectendes.

5. Fructificatione præsente.

6. Siccande inter papyra sicca.

7. Citissime, vix ferro calido.

8. Prelo modicè compresso.

9. Adglutinanda ichthyocollâ.

I0. in folio semper asservand

I. unica tantùm in pagina.

12. plagula non alliganda.

I3. Genus supra adscribendum.

14. Species, et Historia a tergo.

15. Congeneres inter pbily.ram reponendx.

16. Disponendxe ad Msibodum.

12. DESCRIPTORES (3) adumbrationes (325) vegetabilium exhibuerunt.

EXOLET RE:Brunfelsius, Tragus, Dorstenius, Lonicerus, Ruellius, Durantes, Carricbterus, Thurneiserus, Turnerus, Salomon. USITATÆE: Mattbiolus, Cordus (Val.), Fuchsius, Clusius, Dodoneus, Lobelius, Tabernemontarus, Gerbardus, Parkinsonus, Dalechampius.

UNIVERSA LES: F. D.xuhinus cumCherlern, Morisonus, Kajus

CHARACTERISTICAE: Hortus cliffortianus, Hallerus, Flor: Zeylanica.

MENSURATE: Tournefortius, Feuillicus.

PARTIALES in Muscis: Dillenius.

Graminibus: Scheucbzerus, C. Baubinus.

Filicibus americanis: Plumierus.

Officinalibus: Pometus, Valentinus, Godofredus. A 4 EXO- 
EXOTIC $\$$ in Indicis: Rherde, Rumpfus.

Americanis: Plumierus, Sloaneus, Hernandez.

13. MONOGRAPHI (8) Vegetabile unicum opere singulari prosecuti sunt.

Ut eò accuratius constent omnia in particulari casu. Nature curiosorum institutum laudandum.

SELECTA in his pauca sunt.

Dillenii Mesembryanthema. Boerhaavii Protece.

Kæmpferi Thea.

Commendanda Succulenta, necnon amplissima genera, 8. gr . Euphorbium.

Præcipua in hoc genere sunt:

Halleri Allium.

Veronicre alpine.

Pediculares.

Breynii Ginseng.

Lafitau Panas.

Bradley Aloe.

Hellwing Pulsatilla.
Nostri Musa.

Betula nana.

Ficus.

Passifora.

Anandria.

Acrosticum.

Senega.

Lign. colubrnium.

Splachnum.

14. CURIOSI (8) Vegetabilia rariora proposuerunt. Consulendi Auctores in plantis rarioribus sunt. EUROP EAA. Gmelini Sibrrica.

Linnæi Lapponica.

Buxbaumii orientales.

Loeselii Prussica.

Vaillantii Parisina.

Raji Anglica.

Columnæe Ecphrasis.

Bocconis Museum.

Aldini, Triunfetti,
Ammanni Ruthenica.

Halleri Helvetica.

Tournefortii orientales.

Mentzelii pugillus.

Magnolii Monspeliaca.

Tilli Pisana.

Barrelierii Icones.

Alpini Rariora.

INDICA: Dillenii Hortus Elthamensis. Martyni Centuriee. Hermanni Hort.Lugd.Eat.CommeliniHort.Amst. Breynii Centurice, et Prodr. Hermanni Paradisus. Dodartii Historia plant. Plukenetii Phytographia.

MUSEA, quæ e triplici Naturæ Regno constant: Sebre Thesaurus. Catesbrei Carolina. Petiveri Gazophylacium. Anonym.Petropolitanum. ADO- 
15. ADONIDES (8) Vegetabilia sativa cujusdam Horti sistunt.

HOR'TI ACADEMICI in Europa sunt :

Patavinus I 540. Parisinus $1626 . \quad$ Upsaliensis 1657. Pis.unus I 547. Oxoniensis 1683 . Brolinensis.

Eononiensis I 547. Leydensis $1677 . \quad$ Lipsiensis 1580. Morspeliensis 1 598. Amstelodamensis I 686. Gottingensis.

PUBLICOS inter Hortos eminent: Ulirajectinus 1638 . Wittembergensis.
Miggnolii Monspeliensis.
Hermanni Leydensis.
Boerhavii Leyciensis.
Royeni Leydensis.
Tilli
Volkameri
Halleri
Linnei
Pisanus.
Norimbergensis. Gœttingensis. Upsaliensis.

PRIVATI Horti primarii sunt:

Linnei Hortus Cliffortianus. Moebringii Hortus proprius. Morison H. Blesensis.

Thraus Cat. Carolsruhensis.

I6. FLORIST E (8) enumerant vegetabilia spontanea certi alicujus Loci.

Enumeratio sit systematica, ut etiam absentes intelligantur: sint presentes cum Loco, Solo, Tempore, Nominibus indigenis.

PRIMARII sunt Floræ:

\begin{tabular}{|c|c|c|}
\hline Ginelini & Sibirica. & Dalibardi Parisina. \\
\hline Halleri & Helvetica. & Guettardi Stampensis. \\
\hline Linnai & $\begin{array}{l}\text { Suecica. } \\
\text { Lapponica. }\end{array}$ & $\begin{array}{ll}\text { Magnolii } & \text { Monspeliaca. } \\
\text { Ruppii } & \text { Jenensis. }\end{array}$ \\
\hline $\begin{array}{l}\text { Raji } \\
\text { Vaillar }\end{array}$ & $\begin{array}{l}\text { Anglica. } \\
\text { Parisina. }\end{array}$ & $\begin{array}{l}\text { Dillenii Gissensis. } \\
\text { Gostschedii Prussica. }\end{array}$ \\
\hline
\end{tabular}

17. PEREGRINATORES (8) dissitas Regiones plantas investigandi causa adierunt.

PRASTANTIORA sistunt in Botanicis:

Scbeucbzerii itinera alpina.

Calceolarii, et Ponce Montis Baldi.

Ray's Trawels, and Voyages.

Tournefort Voyage de Levant.

Shawii Cat. plantarum Africæ.

Alpini Egypti.

Bellonius, Rauwolfius. 
Ticmpfer: Amcenitates.

Marcgruvii, et Pisonis Brasilia.

Feuillée Perú.

Hernandez México.

Cornuti Canadá.

SELECTIORA sunt Botanica:

Indica: Rheede Malabarica.

Dumanni Thesaurus Zeylanicus.

Linnei Flora Zeylanica.

Rumpfii Anboina.

Kampferi Japonica.

Americana: Sloane Jamayca.

Plumieri Americaina.

Gronovii Flora virginica.

18. METHODICI ( 7 ) de Dispositione (VI), et inde facta Denominatione (VII) vegetabilium imprimis laborarunt; suntque Pbilosopbi(19), $S y$ stematici (24), Nomenclatores ( $\left.3^{8}\right)$.

19. PHILOSOPHI (r8) Scientiam Botanicam demonstrativè ex principiis rationalibus in formam scientiæ reduxerunt: ut Oratores (20), Eristici (21), Physiologi (22), Institutores (23).

Theoretici hi Botanici dicentur.

Canones, et regulæ in Botanicis his debentur. Empirica cognitio erat veterum res herbaria.

20. ORATORES (I9), quæcunque Scientiam doctè ornant, proposuerunt.

ORATIUNCULÆ.

Hellwvigii Botanices nobilitas.

Triumfetti Prolusio.

Commelini Oratio.

PHYSICALIA.

Biberg Oeconomia Naturæ.

Scderberg Curiositas Naturalis.

EMBLEMATA.

Camerarii Emblemata.

Mylii Hortus Philosophicus. 
2r. ERISTICI (19) in Botanicis scriptis publicis litigarunt.

\section{BELLA SYSTEMATICA.}

Tournefortil Elementa - - -

Chomel Responsum.

RaJi Sylloge $\ldots \ldots$ - Rivini Epistola.

*** ***

Raji Epistola.

Raji varix Methodi.

Dillenii Judicium.

- - Tournefort optima methodus.

Dillenii Examen.

$$
\text { - - - Rivini Responsio. }
$$

LINNEI Metbodus

$$
\text { *** }
$$

- - - Siegesbeck Epicrisis.

Browallii Examen.

Gleditsch Consideratio.

$$
\begin{gathered}
\text { - } \text { - Siegesbeck Vaniloquia. } \\
\text { Heisteri Schedulæ. }
\end{gathered}
$$

BELLA REGUNI de Plantis magis cruenta fuere, nec calamo, sed bombardis, et glaciis scripta.

Abies, Cedrus dicta, incitavit Adrianum, ut Hierosolyma destrueretur.

Hrematoxylon incitavit Bellum inter Hispanos, et Anglos, $173^{\text {h. } 1743 \text {. }}$

Ficus incitavit Xerxen contra Athenienses.

Romam contra Carthaginem, suadente Catone.

Balsami de frutice dimicarunt Judæi, et Romani. Plin. XII. Anncardium prælia inter Brasilenses sæpe caussat. Pbanix dissidia crientalium multoties caussavit.

Nyristica Belgas in Indiis armavit.

22. PHYSIOLCGI (IS) Vegetationis Leges, et Sexûs

(V) mysterium in plantis revelarunt.

Millington 1676

Camerurii. (Rud.)- Epistola.

Vaillantii Sermo.

Wablbom Sponsalia nostra plantarum. 
23. INSTITUTORES (in) Regulas, et Carones corsposuerunt.

Jungii Isagoge Phytoscopica.

Nos Fundamenta hre Botanica.

Ludrugii Regnum vegetabile.

Aphorismi Botanici.

24. SYSTEMATICK (I8) plantas in certas Phalanges disposuerunt: qui vel Ortbodoxi (26), vel Heterodoxi ( 25 ) sunt.

25. HETERODOXI Systematici (24) ab alio, quàm fructificationis principio $(164)$ vegetabilia distribuerunt; ut Aipbabetarii, Rbizotomi, Pbyllophili, Pbysiognomi, Chronici, Topopbili, Empirici, Seplasiarii.

Alphabetarii methodo alphabetica.

Rbizotomi a radicis structura: ut Hortulani.

Pbyllophili a foliorum speciebus.

Physiognomi ab habitu.

Chronici a tempore florendi.

Topophili a loco natali.

Empirici ab usu medico.

Seplasiarii secundùm ordinem pharmacopœorum.

26 ORTHODOXI Systematici (24) e Fructificationis vero fundamento ( 164 ) Methodum desumserunt; suntque Universales (27), vel Partiales (32).

Genera observant naturalia.

Genera disponunt secundùm partem aliquam fructificationis. Indicant præsentia; absentia tum per se patent.

27. UNIVERSALES Orthodoxi Systematici (26) genuina methodo omnes vegetabilium classes condiderunt: ut Fructista (28), Corollista (29), Calycista (3o), Sexualista (3r). 


\section{BIBLIOTHECA.

28. FRUCTIST Æ (27) a Pericarpio (86), Semine (86), aut Receptaculo (86) Classes Vegetabilium composuerunt: ut Cesalpinus, Morisonus, Rajus, $K$ nautius, Hermannus, Boerbavius.

Casalpinus (54) Professor Patavinus 1583.

Morisonus (55) Professor Oxoniensis 1680 .

Rajus (59) Presbyter Anglus 1682. 1700.

Knautius (57) Medicus Halensis 1687.

Hermannus (56) Professor Leydensis 1690.

Boerhave (58) Professor Leydensis 1710.

29. COROLLIST (27) a Córolla (86) Petalosa classes distinxerunt: uti Rivinus, Tournefortius.

Rivinus (6I) Professor Lipsiensis 1690.

Heucherus Profesșor Wittembergensis $17 \mathrm{I}$.

Ruppius (6I) Siudiosus Jenensis 1718.

Hebenstreit Professor Lipsiensis $173 \mathrm{I}$.

Ludzvig (63) Professor Lipsiensis I737.

Knautius (62) Bibliothec. Halens. I7 16.

Tournejortius (64) Professor Parisinus 1694.

Plumierus Religgiosus 1703 .

Pontedera (65) Professor Patavin. 1720.

30. CALYCIST FE (27) a Calyce (86) Classes distribuerunt: ut Magnolius, Nos.

Magnolius (66) Professor Monspeliensis: posthumus I 720. Nos (67), 1737 .

3i. SEXUALIST E (27) a Sexu (149) Systema condiderunt: ut $E$ go.

Ego (68) (in Belgio) I 735 .

32. PARTIALES Orthodoxi Systematici (26) unius tantùm Classis Systema composuere: e. gr. Compositorum (77.0rd. 211$)$, Uribellatorum (77. ord: 22), Graminums (77. ord. 14), Muscorum (77. ord.65.66), Fungorum (77. ord. 67).

COM- 
33. COMPOSITORUM (32. IÍz.) Classes exposué runt Vaillantius, et Pontedera.

Vaillantius (70) Demonstrator Parisiensis 1718. Poniedera (70) Professor Patavinus 1720.

34. UMBELLATORUM (32. I I8.) classem instruxerunt Morisonus, tt Artedius.

Miorisonus (7I) Professor Oxoniensis 1672.

sirtedius (71) Cultor Medicinæ, Suecus (1735).

35. GRAMINUM (32) classes composuere Rajus, Montius, Scbeucbzerus, Micbelius, Nos.

Rajus (72) Presbyter Anglus I703.

Montius (72) Professor Bononiens. 1719.

Scheuchzerus (72) Professor Tigurinus 1719.

Michelius (72) botanicus Hetruriæ 1729 .

$\operatorname{Nos}^{\circ}(72)$ in Generibus plantarum 1737.

36. MUSCORUM (32) classem elaboravit Dillenius. Dillenius (73. 74.) P'rofessor Oxoniensis 174r.

37. FUNGORUM (32) classem instruxerunt Dillenius, Micbelius.

Dillenius (75) Med. Gissensis I 7 r 9.

Michelius (75) Botanicus Hetruriæ 1729.

38. NOMENCLATORES (18) de Vegetabilium denominatione solliciti fuere: ut Synonymistice (39), Critici (40), Etymologi (4I), Lexicograpbi (42). 39. SYNONYMIST E $^{3}\left(3^{8}\right)$ diversa nomina Vegetabilium, a Botanicis quondam imposita, cullegerunt.

C. Baubti i Pinax primarius est. Hallerus in variis operibus.

40. CRITICI (38) Nomina generibus, et speciebus . verè propria determinarunt.

Linnei Critica Botanica. 
4r. ETYMOLOGI ( 38 ) radices, et originem nominum

Genericorum eruunt.

Falugii Prosopœia:

42. LEXICOGRAPHI $\left(3^{8}\right)$ nomina diversarum linguarum colligunt:

Mentzelii Indéx multilinguis, s. Lexicon polyglotton.

43. BOTANOPHILI (6) sunt, qui varia de vegatabilibus tradiderunt, licèt ea non propriè ad scientiam Botanicam spectant, ut Anatormici (44), Hor:tulani (45), Medici.(45), Miscellanei (52).

44. ANATOMICI (43) internam structuram vegetabilium contemplati sunt.
Anatomica at Malpighius. Vegetationis leges, Hales.
Physiologica, Feldmin.
Gesnerus. Ludwig.

\section{HORTULANI (43) culturam vegetabilism tra-} diderunt,

- GEOPONICI eardem in usum communem adhibuerunt.

Laurembergii Horticultura: Ferrario Flora.

Elsholtii Horticultura. Bradl y varia anglica.

Ligier gallicè.

Milleri Dictionarium.

ACADEMiCUS Hortus, ejusque instructio. Vid. Naucler.

Diss. de Hort. Upsal.

Partes: Caldarium, Tepidarium, Frigidarium.

Vaporarium, Solarjum, Aprcarium,

Area in Pulvillos artificiose divisa.

Perennis, Annua, Vernalis. Autumnalis,

Ambulacra subdiaria, nuda, tecta,

Sepes, Pergule, , Angiportus.

Instrumenta: Ligo; Sarculum, Rutum,

Iridens, Rastrum, Véctis,

Vebiculum, Carrus, Trulli,

Culter, Hirpax, oncus ambidexter,

Forfex, Gutiurnium,

Crutes, Campana soluris, Otla umbratilis. 
Operationes: Fossio, Irrigatio, Riníatio, Stercoratio, Sarritio, Transmitatio,

Tonsio, Amputatio, Ablactatiogi

Circumpositio, Submersio, Separatio,

- Insectio, Delibratio, Inoculatio.

GEOPONICI curam Agrorum, et Pratorum habent.

Rei Rustice scriptores. \$.9. His curæ sunt:

-Vomer, Occa, Volvulus, Sarculum, Rastrum,

Scamnum, Scrofa, Porca, Sulcus, Lira,

Verruncare, Sarrire, Pastinare, Occare, Resulcare,

Flagellare, Vannare, Cribrare, Molere.

46. MEDICI (43) Vires, et Usum vegetabilium in corpus humanum sectati sunt : ut Astrologi (47), Signatores (47), Chemici (48), Observatores (49), Mecbanici (49), Diactetici (50), Botano-Systematici $(5 \mathrm{I})$.

Didscorides Materia Medica.

Simon Paulli Quadripartitum.

Kanig Regnum vegetabile.

Pomet Histoire des Drogues.

-Valentini Museum museorum.

47. ASTROLOGI (46) virtutem ex astrorum influxu in plantas, SIGNATORES (46) vires a similitudine inter plantæ partem, et corporis partem læsam divinarunt.

Bodenstein.

Papper.

48. CHEMICI (46) Vires vegetabilium ope analyseos ignis extricare crediderunt:

Geoffroy, Tournefort, Tawry.

49. OBSERVATORES (46) a casu, et experientia; MECHANICI (46) a principiis physiologico-mechanicis vires vegetabilium derivarunt.

Observatores Geoffroy Tr. "de Materia Medica.

Hermanni Cynosura."

Boerhavii Historia plantarum.

Halleri Synopsis Helvetica.

Linniai Matería Medica. 
50. DIFTETICI $(45)$ a sapore, et odore vires ingerendorum dijudicarunt.

Quercetanus Diæteticon.

Nonnius Res cibaria.

Eobrens Selecta dixtetica.

Lister in Apicium.

5r. BOTANO-SYSTEMATICI (46) secundùm Classes naturales vires Medicamentorum cautè distinguunt.

Camerarius Convenientia plantarum.

Hasselquist Vires plantarum.

52. MISCELLANEI (46), qui varia de vegetabilibus scripsere in usum aliorum: ut Oeconomi, Biologi, Theológi, Poëtce.

Exconomi Usum plantarum in vita communi tradunt.

Flora Economica.

$P$ an Suecicus

Iter Oelandicum.

Iter Gotlandicum.

Iter Westrogothicum.

Iter Scanicum.

Biologi Panegyrica plerumque exclamarunt.

Theologi Biblicas plaitas explicarunt.

Celsius Hierobotanicum.

Poëtæ: Macer Supposititius.

Strabus Hortulus.

Rapinus Evelyn, Gardiner.

Nevianus Poëmaticum.

Pictorius Pantopolon.

Santolinus Pomona.

Falugius Prosopopceia.

Coulejus VI. libri Plantarum. 


\section{S Y S T E M A T A.}

53. SYSTEMATICIS (24) Orthodoxis (26) nitor, et certitudo scientize Botanices debetur.

Sysi. rat. obs. reget, 3. idem.

Syst. nas. obs. veget. 4. Systematici qui.

Cæsalpinus. Rivinus. Vaillantius. Linnæus.

Morisonus. Knautius. Jussixus. Royenus.

Rajus. Ruppius, Scheuchzerus. Gronovius.

Hermannus. Ludvigius. Dillerins. Gmelinus.

Magnolius. Tournefortius. Michelius.

Boerhavius. Plumierus.

Hallerus. Guettardus.

Gesnerus.

Burnannus.

Wachendorffius.

Gleditschius.

Dalibard.

54. CFEALPINUS (28) est Fructista, et primus verus Systematicus, secundum Corculi (26: VI.), et Receptaculi (86: VII.) situm distribuens.

Arbores corculo ex apice seminis - . - - I. e basi seminis -
Herbx solitariis seminibus
baccis
capulis $\ldots \ldots-\ldots$
Binis seminibus -

Triplici principio fibrosæ - 8 . Bulbosa - 90

Quaternis seminibus - - 10. Pluibus seminibus Anthemides 11. Cichorac. s. Acanacee 12. flore Communi - 13. folliculis - - 14 . flore, fructuque carentis- is.

55. MORISONUS (28) est Fructista cum Physiogno. mis (25), et Curollistis (29) conspirans.

Lignosæe Arbores _......... Frutices - . - . . . 2. Suffrutices - ....... 3 . 
Harbacex Scandintes - - - . - . 4 .

Leguminosa - - - - - - 5 . Siliquose - - . - . - 6 . Tricupsulares - - - - - 7 . a numero capsubstim dicta - - - 8. Corymbifire _ - $\ldots+\ldots$ - 9 . Lactescentics, s. Papposa - - - I0. Cubmifera - . - . - . - II.

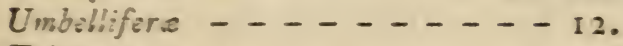
Tricocia - - . - . - - 13 . Gabajic - - - - - - - I 1 . Multicapsubares - - - - - - 15 . Baccifer - . - . - . - I 6 . Capillares - - - - - - 17. Heteroclite. - - - - - - IS.

56. HERMANNUS (28) est Fructista, secundùm fructum Gymnospermum (200), et Angiospermum (200) numerans.

Herbx Gymnospermæ monospermx Simplices

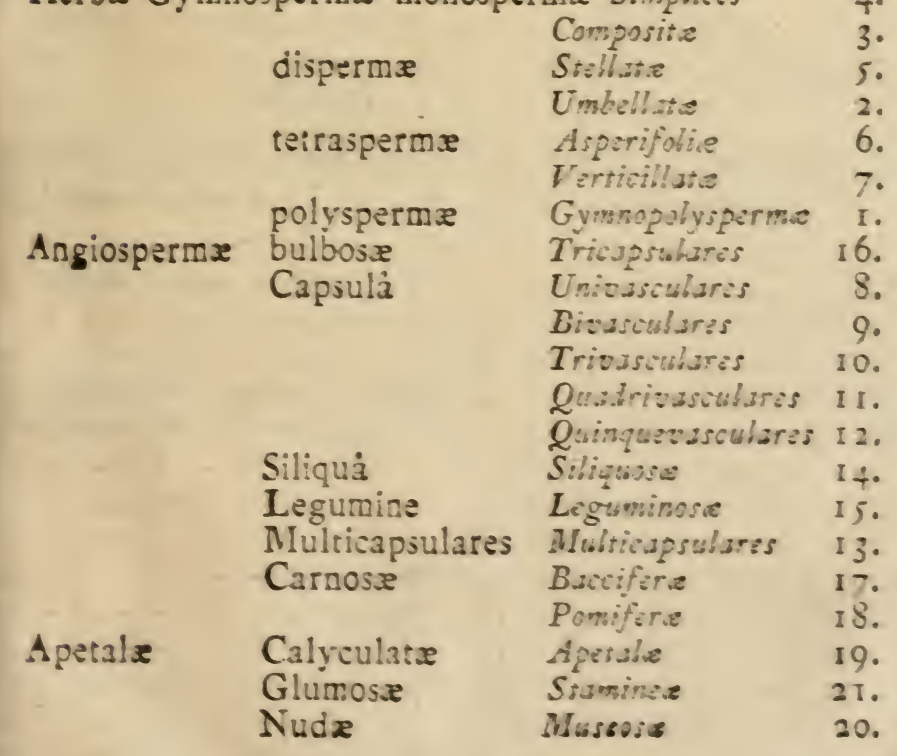




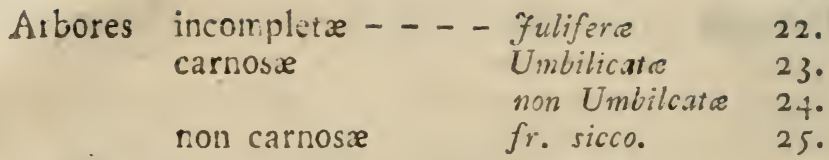

57. KNAUTIUS (Christoph.) (28). Rajanum (59). Syitema inversum adoptavit.

Herbx Petalosæ fructu carnoso Baccifere

nembranaceo Munopetalce

I.

Tetrapet. Regulares 3.

Tetrupet. Irregulares 4.

Pentupetale

Hexupetalce

5 .

Polypetala

Multicapsulares

fructu nudo Gymnospernie

6.

Solida

7.

8.

Fappose

9.

I 0.

Hertæ apetalæ $\ldots \ldots . .-$ Apeialre

I I.

Staminere

I2.

Inconspicuce

13 .

Imperfecte

Lignosæ - - - - - - Arbores

I 4 .

15 .

16.

17.

58. BOERHAAVIUS (28) Systema Hermannianum $\left(5^{6}\right)$ cum Rajano (59. $\beta$ ), et Tournefortiano (45) conciliavit.

Sulmarine - . - - I. Gymnodispermæ Umbelli-

Terrestres - - - - 2.

Capillares - . - . 3 .

Gymnopolysperme - - 4 .

Gymrotetraspermæ $V \epsilon r-$

ticillate-
Asperifol. 13.
Tetrapetal. 14.

ferce - . - . - 5.

Stellatice II.

Gymnomonosp. simplices 6 . Planipetale 7. Radiatre 8.

N.udae 9.

Cispitate I0.

MIonangice - . - - I 5. Bacciferce - . - . - 25. Diangie - - - - 16. Pomifere - - - - 26. Triangice - - - - - 17. Apetale _. - - - 27. Tetrangice - - - - 18. Monocotyled. Dracteatee 28. Pentangice - - - - 19. Apetale 29. Polyangice - . - - 20. Arbores Monocotylewories; 0 . 
Multisiliquose. .....2 $\mathrm{r}$.

Siliquose........22.

Tetrapetale cruciformes. 23.

Legumirose .......24.
Arbores Apetala....3 I. Amentacer. . 32 . Monopetale. 33 . Rosacer... 34 .

59. RAJUS (28) e Fructista (28) Corollista (29) evasit.

a. Methodus propria. Arbores .......... r. Frutices..........2. Hb. Imperfectre. . . . . 3. Flore carentes.....4. Capillares......... St.uminze........6. Gymnomonosperme ...7. Umbellatce .......8. Verticillate.......9. Asperifolie ....... Io. Stellate........ II. Pomifere......... I2. Bacciferc........ I3. Multisibiqure....... I 4 . Monopet. uniformes .. Is. Monopet. difformes . . I6. Tetrapet. siliquose . I I7. Tetrapetale siliculosice I8. Papilionacere..... I9. Pentapetale...... 20. Frumenta.......2 $\mathrm{r}$. Gramina.......22. Graminifolic .....23. Bubbose.......24. Bulbosis affines ... . 25 . b. Methodus EMENDATA. Herb. Submarinæ. ...... . Fungi ........2. Musci ............ 3 . Capillares .......4. Apetalæ......... Planipetalx ...... 6 . Discoidex.......7. Corymbiferæ .....8. Capitatz .......9. Solitario semine ... I 0. Umbelliferæ....... I I. Stellatæ ........ I 2 . Asperifoliz....... I3. Verticillatæ...... I 4 . Polyspermæ...... Is. Pomiferx........ 6 . Bacciferx....... I7. Multisiliquæ ..... I 8. Monopetalx ...... I9. Di-tripetala .....20. Siliquosæ ......2 I. Leguminosx .....22. Pentapetala .....23. Floriferx ......24. Staminex ......25. Anomalz......26. Arundinacex ....227. Árb. Apetalx .......28.

Fr. umbilicato ...229. Fr. non umbilicato. 30 . Fr. sicco........ 3 r. Fr. siliquoso..... 32 . Anomalæ.......33. 
60. CAMELLUS secundùm valvulas (86) Pericarpii (85: V.) plantas disponere tentavit.

$\begin{array}{clc}\text { Pericarpia } & \text { Afora. } & \text { Tetrafora. } \\ & \text { Unifora. } & \text { Pentafora. } \\ & \text { Bifora. } & \text { Hexafora. } \\ & \text { Trifora. } & \end{array}$

61. RIVINUS (29) est Corollista, secundùm Regularitatem, et Numerum Petalorum cum Triplici fructu.

Regulares Monopetali I. Irregulares Monopetali II. Dipetuli 2. Dipetali 12. Tripetali 3. Tripetali I3. Tetrapetali 4. Tetrapetali I 4 . Peniapetali 5. Pentapetiali i 5. Hexapetali 6. Hexapetali 16. Polypetali 7. … Polypetali 17.

Compositi ex flosc. Regularibus 8. Incompleti Imperfecti 18. Reg. et Irreg. 9.

Irregularibus io.

Triplex fructus: sc. nudus, Pericarpium (siccum), carnosum. RUPPIUS Systema Rivinianum in Compositis emendavit.

62. KNAUTIUS (Christian. Corollista) (29) Rivinianum (6I) Systema inversum, Numerum scilicet Regularitati præferendo, suum fecit.

Monopetali Uniformes I. Difformes aggregati Uniformes 3. Difformes

Dipetali Uniformes 6. Difformes

Tripetali Uniformes 8. Difformes

Tetraperali Uniformes $10 . \quad$ Difformes

2.

Pentapetali Uniformes I 2.

Hexapetali Uniformes i4.

Difformes

Polypetali Uniformes 6 .

Difformes

4.

5.

Negavit flores apetalos, seminaque nuda dari.

63. LUDWIGIUS Methodum Rivini (6r) cum Linnæana (68) combinavit.

Classes Rivini ex Regularitate, et Numero Petalorum. Crdines at Methodo sexuali in singula classe; idem $I V$ edelius. 


$\begin{array}{ll}\text { Monantherce } & \text { Monostylæ. } \\ \text { Dianthere } & \text { Distylæ. } \\ \text { Trianthere } & \text { Tristylæ. } \\ \text { Pentantherce } & \text { Tetrastylæ } \\ \text { Decanthere \&c. } & \text { Polystylæ \&c. }\end{array}$

64. TOURNEFORTIUS (29) est Corollista secundùm Regularitatem, et figuram cum duplici Situ Receptaculi (86) floris.

Simplices Campaniformes t. Compositi Flosculosi .... I 2. Infundibuliformes 2. Anomali ...... 3 . Labiati ........ 3. Apetal hadiani.... Cruciformes....5. F. Flore carentes..... I6. Rosacei .......6. Flore, fructuque car.17. Umbellati..... 7. Arbores: Apetali...... I8. Caryophyllei...8. Amentacei....... 19. Liliacei...... Mo. Monopetali......20. Papilionacei... ro. Rosacei.......2 21. Anomali ..... II. Papilionacei.....22.

Ordines a Pistillo, aut Calyce in fructum abeunte.

65. PONTEDERA Tournefortianum (64) cum Riviniano (6I) combinare tentavit.

Incertæ . Incerta ....... $\mathrm{x}$. Floribus destituta 2. Gemmis carentes Imperfecti $\ldots 3$. Anomali ........ 4 . - Labiati ........ 5. Campaniformes .. 6 . Hypocrateriform. .7. Rotati ........8. Infundibuliform..99. Flosculosi..... ro. Lingulati...... I I. Radiati capitulis 12.
Anomali ....... 3 .

Papilionacei .... I 4 .

Liliacei ....... I5.

Caryopbyllici.... I6.

Cruciformes .... I 7 .

Umbellati..... I 8 .

Filamentosi .... I9.

Gemmiferx Filamentosi. 20.

Apetali .......2 $\mathrm{I}$.

Anomali...... 22.

Campaniformes .. 23.

Rotati......24.

Infundibuliformes 25 .

Papilionacei. . . 26.

Rosacei......27. 
66. MAGNOLIUS (30) est Calycista cum Fructistis (23) combinatus.

Herb. calyce Externo includente fiorem ignotum I.

staminium

2.

monopetalum 3 . polypetalum 4 .

compositum

sustinente florem monopetalum 6. polypetalum 7 . Interno tantùm.............. 8. Externo, internoque, flore monopetalo 9. di-tripetalo Io. tetrapetalo II. polypetalo I2.

Arb. calyce Externo tantùm ................ I 3 . Interno tantùm .............. I 4 . - Externo, Internoque simul. ......... I5.

67. NOS.(30) Methodum Calycinam secundùm Calycis species (86) exaravimus.

Spatbacei

Glumosi

Amentacei

Umbellati

Communes

Duplicati

Floribundi

Coronati

Anomali
I. Difformes ............ I0.

2. Caduci................. II

3. Persistentes uniform monopet..... I 2. 4 .

5.

6. polypet...... I 3 . difformes monopet. . . . . I 4 . polypetali..... I 5 . 7. Incompleti........... 16. 8. Apetali................ I7. 9. Nudi. .............. I 8 .

68. EGO (3I) Sexuale Systema secundùm Numerum, Proportionem, et Situm Staminum cum Pistillis elaboravi.

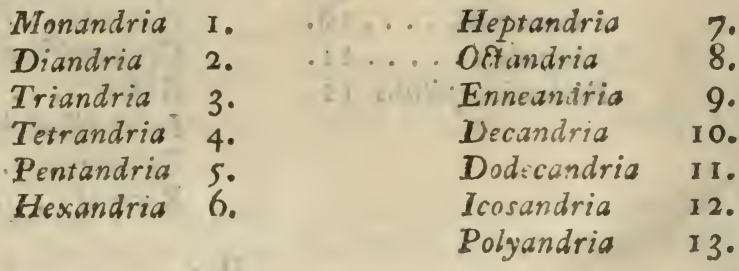




$\begin{array}{ll}\text { Didynamia } & 14 . \\ \text { Tetradynania } & 15 . \\ \text { IIonidelpbia } & 16 . \\ \text { Diadelphia } & 17 . \\ \text { Polyadelpbia } & \text { I8. } \\ \text { Syngenesia } & 19 .\end{array}$

Conandria 20. Minocia $2 \mathrm{I}$. Diacia 22. Poljgamia 23. Crypiogamia 24.

69. NATURALEM Methodum in Cotyledonibus, Calyce, Sexu, aliisque Royemus pulchrè, Hallerus eruditè, $W$ achendorffurs græcè quæsiverunt. *. ROYENUS Prof. Leydensis 1740 .

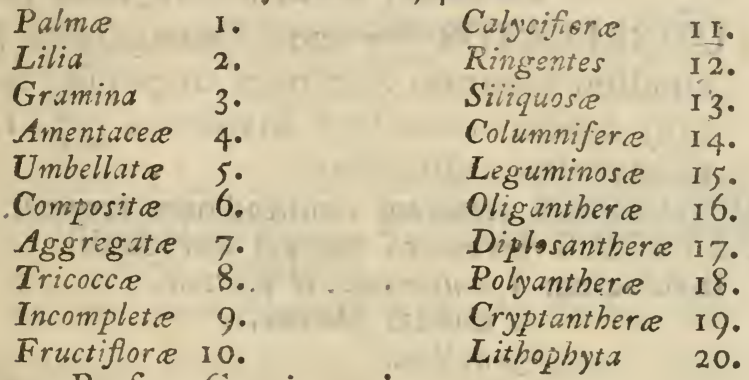

b. Hallerus Profes. Goettingensis I742.

Fungi . ........ I. D plostemones. . . . . . 9 . Musci......... 2. Isostemones ........ Io. Epiphyllosperma..... 3. Mejostemones....... II. Apetale.......4. Staminibus sesquialteris. . 12. Gramina. ......5. Staminibus sesquitertiis. . I3. Graminibus affinia ... 6. Staminibus qualuor, ringenMonocotyled. Petaloidere 7. tes......... I4. Polystemones ......8. Congregata....... 15.

6. WACHENDORFFIUS Prof. Ultrajectinus 1747.

Gymnosperme. . . . . I. Cylindranthere...... II. Homojodiperiunthe ... 2. Monoperiantha. . . . I 12. Anomojediperisuthe... 3. Monoplythanthe..... I3. Palloplostemonopetalce. 4. Diphyihanthe...... 4 . Anisostemorapetilia... 5. Acalyces........ 15. Cylindrobasiorlemones. . 6. Calycine ........ i6. Dimacrostemones.... 7. Spathacea ....... I7. Tetrumacrostemiones. . 8. Glumose........ 18. Distimonopleunthere.. 9. Cryptanthe....... I9. Eleutberinibara .... 10. 
70. COMPOSITOS Flores (77:2r.) Vaillantius (33) a Calyce (86. r.), Receptaculo (85. 7.), et Seminum Coronula (86.6.) distinguit; PONTEDERA (33) de Compositis. Vaillantio theoreticè accedit in ordinibus.

VAILLANTIUS I7I8.

CLASSES. Cynarocephali, Corymbiferi, Cichoracei, Dipsacei. ORDINES. Calyx: simplex, imbricatus, calyculatus.

Receptaculum: nudum, pilosum, paleaceum. Coronula: nulia, pilosa, plumosa.

₹. UMBELLATOS $(77: 22$.$) Morisonus (34) se-$ cundùm Figuram Seminum disposuit; ARTEDrus (34) autem secundùm Involucra (77 . I.) trifariam primus distribuit.

MORISONUS seminum similitudinem respexit. ARTEDIUS involucra tantùm introduxit.

Involucrum I universale, et partiale.

2 tantùm partiale.

3 nullum.

72. GRAMINA (77: 13, I4) Rajus (35) secundùm affinitatem cum Cerealibus disponit, cui assentit Monti, et splendidè elaboravit Scheuchzerus (35). Mrchelius (36) secundùm Glumas simplices, et compositas; Nos (35) secundùm Sexum Gramina retulimus.

RAJI, MONTI, SCHEUCHZERI Classes sunt.

Spicata Triticea

Hordeacea

Secalina

Loliacea

Panicea

Phalaroidea

Alopecuroidea

Typhoidea

- Myosuroidea

Echinata

Cristata

Anomala
Dactyloidea

Paniculata Simplicia mutica.

Composita.

Affines: Linagrostis

funcoides

Funcus

Canna

Scirpus

Cyperus

Cyperoides. 
73. MUSCOS $\left(77: 6_{5}\right)$ stupenda industria detexit, et absolvit Dillenius (36).

Calyptrati.

Calyptra destitut:.

74. AlGAS (77:66) Dillenius ex Textura, MICHELIUS secundum flores disposuit.

75. FUNGOS (77:67) Drllenius (37) secundùm Pileos, Michelius (37) secundùm fructificationes dispescuit.

DILLENII divisio secundùm Pileos, unde

Lamellosi,

Porosi,

Echinati.

76. LITHOPHYTA olim relicta Plutoni, Marsillius Flore imperio subjecit, at PEYSONELLUs eadem Faunce Regno restituit.

Amon. acad. 80. ratio Peysonelli I727. B. Jussici I77I.

77. METHODI NATURALIS Fragmenta studiosè inquirenda sunt.

Primum, et ultimum hoc in Botanicis desideratum est.

Natura non facit saltus.

Plantæ omnes utrinque affinitatem monstrant, uti Territorium in Mappa geographica.

Fragmenta, qux ego proposui, hæc sunt:
I. PIPERIT \&.
Phœenix
Arum
Cycas
Dracontium
Calla
3. SCITAIIINA.
Acorus
Saururus
Pothos
Piper Musa
Thalia
Phytolacca.
2. PALMIE.
Corypha
Alpinia
Costus
Canna
Maranta
Amomum
Borassus
Curcuma
Kæmpferia.
Coccus
Chamærops
4. ORCHIDEE.
Orchis
Satyrium
Serapias
Herminium
Neottia
Ophrys
Cypripedium
Epidendrum
Limodorum
A rethisa.
5. ENSATÆ.
Iris
Gladiolus
Antholyza
Ixia 
Sisyrinchium

Commelina

Xyris

Eriocaulon

Aphylianthes.

6. Tripetalodez.

Butomus

Alisma

Sagittaria.

7. DENUDAT E.

Crocus

Gethyllis

Bulbocodium

Colchicum.

8. SPATHACEÆ. I 4. GRAMINA.

Ieucojum

Galanthus

Narcissus

Pancratium

Amaryllis

Crinum

Hzemanthus.

9. CORONARIAE.

Ornithogalum

Scilla.

Hyácinthus

Asphodelus

Anthericum

Polianthes.

I o. LILIACEA:

Liliứn

Fritillaria

Tulipa

Erythronium.

I . MURICATE.

Bromelia

Renealmia

Tillandsia

Burmannia.

r2.COADUNATE.

Annona.

Liriodendrum
Mignolia

Uvaria

Michelia

Thea.

I 3.CALAVIARIE.

Bobartia

Scirpus

Cyperus

Eri sphorum

Carex

Schenus

Fligellaria?

Juncus?

Scheuchzeria?

Zea

Coix

Ischæmum

Cornucopix

Nardus

Saccharum

Zizanía

Phalaris

Phleum

Alopecurus

Panicum

Milium

Agrostis

Lagurus

Dactylis

Holcus

Melica

Aira

Poa

Briza

Uniola

Cenchrus

Cynosurus

Bromus

Festuca

Avena

Arundo
Lolium

Triticum

EEvilops

Secale

Hordeum

Elymus

Anthoxanthum

Oryza.

I5. CONIFER E.

Abies

Pinus

Cupressus

Thuja

Juniperus

Taxus

Ephedra.

I6. AMENTACERE:

Pistacia

Myrica

Alnus

Betula

Silix

Populus

Platanus

Carpinus

Corylus

Juglans

Quercus

Figus.

I7. NUCAMENTACE仼.

Xanthium

Ambrosia

Parthenium

Iva

Micropus

Artentisia?

I8. A GGREGA.

T FE.

Statice

Protea

Leucadendros 


\section{Habenstretia}

Brunia

Cephalanthus

Globularia

Scabiosa

Knautia

Dipsacus

Vileriana

Morina

Loerhavia

Cirrzen?

19. DUMIOSAL.

Viburnum

Tinus

Onulus

Sambucus

Rondeletia

Bellonia

Maurocenia

Cassine

Rhus

Cotinus

Celastrus

Evonymus

Ilex

Tonex

Prinos

Callicarpa

Lnwion:a.

20. SCABRIDAE.

Filus

Iorstenia

P. rietaria

I'rica

Cannabis

Acnida

Hunulus

Ninrus.

2 I. CUNIPOSITI.

a. Semifrosculnsi.

Pienauthes

Lactuca
Chondrilla

Hieracium

Crepis

Andryala

Hypochxris

Picris

Hyoseris

Leuniodon

Scorzonera

Tragopogon

Solymus

Sonchus

Lapsana

Cichorium

Carananche

Elephantopus.

b. Captati.

Ectinops

Sphrranthus

Gundelia

Arctium

Serratula

Onopordum

Carduus

Cynara

Carthamus

Carlina

Cniclis

Atraciylis

Centaurea

Corymbium.

c. Corymbiferi.

Sioebe

Santolina

Chrysocoma

Tanacetum

Ileinia

S ahelina

Xeranchemum

Ginaphalium

Cal perium

Conyza
Tarchonanthus

Baccharis

Erigeron

Tussilago

Doronicum

Sulidago

Senecio

Inula

Aster

Gerbera

Oihonna

Chrysanihemum

Matricaria

Buphthalmum

Anacyclus

Cotula

Anthemis

Achillaxa

Eriocephalus

Helenia

Aretotis

Bellis

'Tagetes.

d. Oppositifoliz.

Helianthus

Rudbeckia

Coreopsis

Bidens

Verbesina

Sigesbeckia

Milleria

Silphium

Eupatorium

Ageratum

Ostenspermum

Calendula?

Chryscgonum?

Melampodium?

Tridax?

Telragonotheca?

22. UVBULLAT

Eryngium 
Arctopus

Hydrocotyle Sanicula

Astrantia

Tordylium

Caucalis

Artedia

Daucus

Ammi

Bunium

Conium

Selinum

Athamanta

Peucedanum

Crithmum

Cachrys

Ferula

Laserpitium

Ligusticum

Angelica

Sium

Bubon

Sison

Oenanthe

Phellandrium

Cicuta

Coriandrum

Ethusa

Bupleurum

Scandix

Chærophyllum

Seseli

Imperatoria

Heracleum

Thapsia

Pastinaca

Smyrnium

Anethum

Carum

Pimpinella.

Egopodium

Apium
Anisum

Lagoecia.

23. MI U L T ILIQUÆ.

Pronia

Aquilegia

Aconitum

Delphinium

Garidella

Nigella

Isopyrum

Helleborus

Caltha

Ranunculus

Myosurus

Adonis

Anemone

Hepatica

Pulsatilla

Atragene

Clematis

Thalictrum.

24. BICORNES.

Ledum

Azalea

Rhododendrum

Andromeda

Clethra

Erica

Blæria

Myrsine

Memecylum

Santalum

Vaccinium

Arbutus

Royena

Diospyros

Melastoma

Pyrola

25. SEPIARIE.

Nyctanthes

Jasminum
Ligustrum

Brunsfelsia

Olea

Chionanthus

Phillyrea

Fraxinus

Syringa.

26. CULMINIÆ.

Tilia

Theobroma

Sloanea

Bixa

Heliocarpus

Triumfetta

Bartramia

Muntingia

Clusia

Dillenia

Kiggelaria

Grewia

Corchorus.

27.VAGINALES.

Laurus

Helxine

Polygonum

Bistorta

Persicaria

Atraphaxis

Rheum

Rumex.

28. CORYDALES.

Melianthus

Epimedium

Hypecoum

Fumaria

Impatiens

Leontice

Monotropa?

Utricularia?

Tropsolum?

29. CONTORTI.

Rauwolfia 


\begin{tabular}{|c|c|c|}
\hline Tevetia & Hyoscyamus & 36. COMOS. \\
\hline Cerbera & Nicotiana & Spiræa \\
\hline Plumiera & Atropa & Filipendula \\
\hline Tabernæmontana & Mandragora & Aruncus. \\
\hline Cameraria & Datura & 37. POMACEE. \\
\hline Periploca & Verbascum & Punica \\
\hline Nerium & Celsia & Pyrus \\
\hline Vinca & Digitalis. & Cratægus \\
\hline Apocynum & 34. COLUMNI- & Mespilus \\
\hline Cynanchum & FERE. & Sorbus \\
\hline Ceropegia & Camellia & Ribes. \\
\hline Asclepias & Xylon & 38.DRUPACEE. \\
\hline Stapelia. & Gossypium. & Amygdalus \\
\hline o. RHAEADES. & Urena & Prunus \\
\hline Papaver & Hibiscus & Cerasus \\
\hline Argemone & Turnera & Padus. \\
\hline Chelidonium & Malope & 39. ARBUSTIVA. \\
\hline Bocconia & Lavatera & Philadelphus \\
\hline Sanguinaria & Althæa & Eugenia \\
\hline Actæ: & Alc & Psidium \\
\hline Podophyllum. & Malva & Myrtus \\
\hline .PUT AMINEA. & Melochia & Caryophyllus. \\
\hline Capr & Sida & 40. $C \wedge L Y C$ \\
\hline Brey & Napæa . & THEMA \\
\hline Mori & Waltheria & Epilobium \\
\hline Crateva & Mentzelia & Oenothera \\
\hline Marcgravia. & annia & Jussiæa \\
\hline 2.CAMPANACEI. & Helicteres & Ludwigia \\
\hline Convolvulus & Stewartia. & Oldenlandia \\
\hline Ipoi & 35. SENTICOSE & Isna \\
\hline Polemonium & Rosa & Ammannia \\
\hline anula & Rut & Peplis \\
\hline Roë! & Frag & Lythrum \\
\hline uma & Potentilla & Glaux \\
\hline Trachelium & Tormentilla & R.hexia. \\
\hline Jasione & Sibbaldia & 41. HESPERIDET \\
\hline Lobelia & Agrimonia & Citrus \\
\hline Viola. & Dryas & Siyrax \\
\hline 3. LURIDA. & Geum & Garcinia. \\
\hline Capsiccum & Comarum & 42. CAR IOPHIL \\
\hline Solanum & Aplanes & L. \\
\hline Physalis & Alchemilla. & Dianthus \\
\hline
\end{tabular}


Saponaria

Drypis

Cucubalus

Silene

Lychnis

Coronaria

Agrostema

Frankenia

Alsine

Cerastium

Holosteum

Arenaria

Pharnaceum

Sp:rgula

Sagina

Moerhingia

Scleranthus.

43. ASPERIFOLIEE.

Tournefortia

Cerinthe

Symphytum

Pulmonaria

Anchusa

Lithospermum

Myosotis

Heliotropium

Cynogiossum

Asperugo

Lycopsis

Echium

Borrago.

44. STELLAT F.

Anthospermum

Ribia

Aparine

Galium

Valantia

Spermacoce

Sherardia

Asperula

Crucianella
Hedyot is

Phyllis

Spigclia

Lippia

Diodia

Knoxia

Cornus?

Coffea.

45. CUCURBITA-47. TRICOCCA. CE无.

Passiflora

Fevillea

Momordica

Trichosanthes

Cucumis

Cucurbita

Bryonia

Sicyos

Melothria

Gronovia?

46. SUCCULENT框.

Cactus

Mesembryanthemum.

Tetragonia

Aizoon

Sempervivum

Seduin

Cotyledon

Rhodiola

Crassula

Tillea

Anacampseros

Portulaca

Claytonia

Chrysosplenium

Heuchera

Saxifraga

Mitella

Penthorum

Geranium
Linum

Oxalis

Zygophyllum

Adoxa

Fagonia

Tribulus

Neurada?

Averrlioa.

Cambegia

Euphorbia

Dalechampia

Clutia

Andrachne

Phyllanthus

Osyris

Croton

Tragia

Acalypha

Cneorum

Jatropha

Ricinus

Cliffortia

Mercurialis

Hernandia

Sterculia

Carica

Hura. 48.INUNDAT ÆE.

Hippuris

Elatine

Proserpinaca

Myriophyllum

Ceratophyllum

Potamogeton

Zanichellia

Ruppia

Zostera

Sparganium

Typha.

49. SARMENTACEE. 


\section{Cissus \\ Vitis \\ Hedera \\ Panax}

Houstonia

Aralia

Ruscus

Asparagus

Medeola

Uvularia

Convallaria

Gloriosa

Rajania

Dioscorea

Smilax

Tamus

Menispermum

Cissampelos

Asarum

Aristolochia

Hippocratea?

so. TRIHILAT E.

Cardiospermum

Paullinia

Sapindus

Staphylæa

Malpighia

Bannisteria

Begonia

Acer

Triopteris

Esculus

Berberis?

I. PRECIA.

Primula

Androsace

Diapensia

Cortusa

Dodecatheon

Soldanella

Cyclamen.

2. ROTACEE。
Gentiana

Exacum

Chironia

Swertia

Lysimachia

Anagallis

Trientalis

Centinculus

Hottonia?

Samolus?

53. OLERACEAE.

Spinacia

Blitum

Pieta

Galenia

Atriplex

Chenopodium

Rivina

Petiveria

Herniaria

Illecebrum

Polycnemum

Axyris

Achyranthes

Amaranthus

Gomphrena

Celosia

Ceratocarpus

Corispermum

Callitriche

Salsola

Salicornia

Anabasis.

54. VEPRECULF.

Rhamnus

Sideroxylum

Chrysophyllum

Lycium

Ceanothus

Philyca

Cesirum

Catesbaa
Daphne

Struthia

Gnidia

Passerina

Siellera

Lachnea.

55. PAPILIONACEAE.

Erythrina

Anagyris

Cytisus

Robinia

Achyronia

Genista

Spartium

Ulex

Borbonia

Colutea

Crotalaria

Ononis

Lupinus

Galega

Securidaca

Glyciné

Phaseolus

Dolichos

Clitoria

Pisum

Lathyrus

Vicia

Orobus

Lotus?.

Dorycnilum

Psoralea

Anthyllis

Trifolius

Ervum

Cicer

Coronilla

Ornithopus

Scorpiurus

Hippocrepis 
Eschynomene

Hedysarum

Glycyrrhiza

Medicago

Trigonella

Arachis

Phaca

Astragalus

Biserrula

Tragacantha

Indigofera

Amorpha

Dalea.

56. LOMENTA-58.VERTICILCE正.

Sophora

Cercis

Bauhinia

Parkinsonia

Cassia

Poinciana

Tamarindus

Guilandina

Adenanthera

Hæmatoxylon

Cæsalpina

Mimosa.

57. SILIQUOSÆ.

Myagrum

Anastatica

Subularia

Lepidium

Cochlearia

Iberis

Thlaspi

Biscutella

Clypeola

Alyssum

Lunaria

Draba

Vella

Cheiranthus
Hesperis

Raphanus

Dentaria

Cardamine

Brassica

Sinapis

Arabis

Turritis

Erysimum

Sisymbrium

Crambe

Isatis

Bunias.

LAT E.

Ajuga

Teucrium

Trichostema

Thymus

Satureja

Clinopodium

Origanum

Lavandula

Hyssopus

Melissa

Horminum

Salvia

Rosmarinus =

'Ziziphora

Monarda

Lycopus

Amethystea

Glechoma

Mentha

Ocymum

Dracocephalum

Nepeta

Betonica

Sideritis

Cunila

Lamium

Galeopsis
Stachys

Melitis

Ballota

Marrubium

Moluccella

Leonurus

Orvala?

Phlomis

Brunella

Scutellaria

Prasium.

59.PERSONAT F.

Cymbaria

Antirrhinum

Rhinanthus

Pedicularis

Bartsia

Euphrasia

Melampyrum

Obolaria

Orobanche

Lathræa

Chelone

Mimulus

Dodartia

Gesneria

Swalbea

Duranta

Columnea

Gerardia

Craniolaria

Torenia

Martynia

Scrophularia

Sesamum

Gratiola

Capraria

Ruellia

Justicia

Barleria

Besleria

Browallia 
Erinus

Buchnera

Tozzia

Verbena

Veronica

Acanthus

Vitex

Volkameria

Clerodendrum

Cornutia

Lantana

Petrea

Bignonia

Citharexylon

Bontia

Halleria

Gmelina

Ovieda? Finetia?

60.PERFORAT

Hypericum

Ascyrum

Cistus

Telephium

61.STATUMINA-

$T$ 死.

Ulmus

Celtis

Bosea.
62. CANDELARES Mnium

Rhizophora

Mimusops

Nyssa.

63. CYMIOSE.

Lonicera

Mitchella

Loranthus

Ixora

Morinda

Chinchona?

64. FILICES.

Ophioglossum

Osmunda

Pteris

Trichomanes

Adiantum

Lonchitis

Asplenium

Hemionitis

Polypodium

Acrostichum.

65. MUSCI.

Lycopodium

Porella

Fontinalis

Sphagnum

Splachnum

Phascum
Polytrichum

Bryum

Hypnum

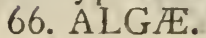

Marchantia ,

Jungermannia

Anthoceros

Lichen

Blasia

Riccia

Ulva

Tremella ins?

Spongia

Conferva

Chara

Fucus.

67. FUNGI.

Agaricus

Boletus

Hydnum

Phallus

Elvela

Clavaria

Clathrus

Peziza

Lycoperdum

Byssus

Mucor.

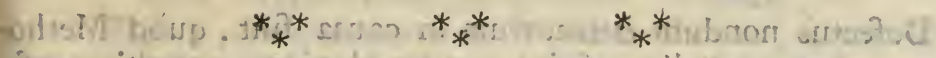

68. VAG死, et etiannum incerte, sedis.
Pinguicula
Penæa
Collinsonia
Polypremum
Buffonia
Budleja
Hirtella
Montia
Plantago
Mollugo
Siphonanthus
Scoparia
Pavetta
Avicennia
Ptelea
Trapa
Elæagnus
Brabejum
Hamamelis
Cuscuta
Coldenia
Menyanthes
Hydrophyllum
Sirychnos
Theophrasta
Patagonula
Plumbago 


$\begin{array}{ll}\text { Phlox } & \text { Hydrangea } \\ \text { Genipa } & \text { Cherleria } \\ \text { Conocarpus } & \text { Mesua } \\ \text { Mirabilis } & \text { Mammea } \\ \text { Coris } & \text { Calophyllum } \\ \text { Cupania } & \text { Elæocarpus } \\ \text { Itea } & \text { Microcus } \\ \text { Cressa } & \text { Ochna } \\ \text { Nama } & \text { Sauvagesia } \\ \text { Basella } & \text { Vateria } \\ \text { Parnassia } & \text { Chrysobalanus } \\ \text { Suriana } & \text { Plinia } \\ \text { Pontederia } & \text { Nymphæa } \\ \text { Tradescantia } & \text { Calligonum } \\ \text { Aloë Yucca } & \text { Tetracera } \\ \text { Hemerocallis } & \text { Loselia } \\ \text { Richardia } & \text { Limosella } \\ \text { Cordia } & \text { Ovieda } \\ \text { Triglochin } & \text { Cleome } \\ \text { Dodonæa } & \text { Hugonia } \\ \text { Grislea } & \text { Connarus } \\ \text { Jambolifera } & \text { Pentapetes } \\ \text { Guajacum } & \text { Polygala } \\ \text { Cynometra } & \text { Nepenthes } \\ \text { Anacardium } & \text { Pistia } \\ \text { Dictamnus } & \text { Cynomorium } \\ \text { Toluifera } & \text { Liquidambar } \\ \text { Melia } & \text { Najas } \\ \text { Schinus } & \text { Osyris } \\ \text { Th } & \text { T }\end{array}$

Viscum

Hippophaë

Antidesma

Pisonia

Zanonia

Coriaria

Empetrum

Lemna

Marsilea

Isoetes.
A. Hydrocharis Stratiotes Valisneria.

B. Ruta Peganum.

C. Sanguisorba Poterium.

D. Reseda

- Datisca.

E. Ceratonia Gleditsia.

F. Veratrum Melanthium.

G. Selago ... Camphorosma. H. Ophiorrhiza Mitreola.

Defectus nondum detectorum in causa fuit, quòd Methodus naturalis deficiat, quam plurium congnitio perficiet; Natura enim non facit saltus. 


\section{PLANT $Æ$.}

78. VEGETABILIA comprehendunt Familias VII: Furigos, Algas, Muscos, Filices, Gramina. Palmas, Rlantas.

Constant Vegetabilia triplicibus vasis:

I. VASA SUCCosa liquorem vehunt.

2. Utriculi alveolis succum conservant.

3. Traches aërem attrahunt.

Gesneri Diss. de Vegetabilibus de his consulenda.

I. Fungi;

2. ALG

3. Muscr; his Anthera absque Filamento, remota a flore Femineo: destituto Pistillo; Semina verò propriâ tunicâ, cotyledonibusque carent.

4. Filices; his Frondes sunt fructificantes averso latere.

5. Gramina ; his Folia simplicissima, Culmus articulatus, Calys glumosus; Semen unicum.

6. PALMIE; his Caudex simplex, apice frondosus, fructificationes in Spadice cum Spathx.

7. PLANTE dicuntur reliqux, qux priores intrare nequeunt familias.

Herbacere quotannis supra Radicem pereunt, cùm Radix omnis herbæ perennis infra terram gemmam proferat. Frutices: Caudex adscendit supra terram absque gemmis. Arbores: Caudex adscendit supra terram cum gemmis.

Gemmx vel distinguunt Frutices ab Arbore, vel nulli limites, cùm magnitudo nihil facit. Pontedera itaque Gemmiparas dixit Arbores.

Indic $æ$ arbores maximæ frutices dicentur, quun in his rarò gemma: adeoque hæc divisio non est naturalis, cùm intra Fruticem, et Arborem nullos limites posuit natura, sed opinio vulgi.

79. Vegetabilium ( $\left.7^{8}\right)$ PARTES, primùm a Tyrone distinguendæ, sunt III. Radix, Herba, Fructificatio. Constat Vegetabile ex Medulla i, vestita Ligno 2, facto ex Libro 3, secedente a Cortice 4, induto Epidermide 5. Medulla crescit extendendo se, et integumenta.

Fibræ medullaris extremitas per Corticem protrusa solvitur in Gem mam imbricatam ex foliolis nunquam renascituris. $\mathrm{C}_{3}$ - Her- 
Herbæ compendium Gemma est, et extenditur in infinitum, doaec Fructificatio imponat ultimum terminum antiquæ vegetationi.

Fructificatio fit, cum folia distinguenda cohærent in Caljecm, quo rumpitur ramuli apex in florem annuo spatio præcociùs, tum Fructus ex medullari substantianequit novam vitam in hoare, nisi prì̀s Staminum essentia Lignea absorpta fuerit ab humore Pistilli. Vide Lafl. de Gemmis.

$V$ cgetabile omne a Radice propagatur continuando.

Fructificatio omnis e Radice per Herbam producta.

Terminztur omne vegetabile Fructificatione ; alioquin vix cessaret crescere.

Nova creatio nulla; sed continuata generatio, cùm Eorculum semiais constat parte radicis medullari.

80. RADIX (79.) alimentum hauriens, Herbamque (8I) cum Fructificatione (IV) producens, componitur Medulla, Ligno, Libro, Cortice; constatque Caudice, et Radicula.

A. Radicula est pars Radicis fibrosa, in quam terminatur Caudex descendens, et qua Radix nutrimentum haurit pro vegetabilis sustentatione.

B. Caudex descendens sub terra sese sensim subducit, et Radiculas (A) profert, a Botanicis ex varia structura variis nominibus distinctus.

1. Perpendicula is, quæ rectâ descendit. Tab. VI. Fig. 129.

2. Horizontalis, quæ sub terra transversim extenditur; Iris.

3. Simplex Tab. VI. fig. I 29. quæ non subdividitur.

4. Ramosa Tab. VI. f. I30. qua in laterales ramos dividitur.

5. Fusiformis Tab. VI. f. I 29. quæ oblonga, crassa, attenuata; ut Daucus, Pastinaca.

6. Tuberosa Tab. VI. f. 128. quæ subrotundis constat corporibus in fasciculum collectis: Preonia, Hemerocallis, Heliantbus, Solanum, Filipendula.

7. Repins Tab. VI. f. I 3 I. quæ longè excurrit, et radiculas hinc inde demittit.

8. Fibrosa constat tantùm Radiculis fibrosis,

9. Premorsa, qua deorsum truncata est, nec attenuato apice terminatur; ut Scabiosa, Plantago, Valeriana.

C. Caudex ascendens supra terram sensim se elevat, gerens sæpius vices Trunci, Herbamque ( 8 I) profert. 
Arbores, Fruticesque omnes itaque sunt Radices supra terram. Ergo Arbor verticaliter inversa e caudice descendente fert Folia, ex adscendente Radiculas.

8I. HERBA (79) est vegetabilis pars, orta a Radice (80), terminata Fructificatione (85), comprehenditque Truncum, Folia, Fulcra, Hybernaculum. Truncus multiplicat herbas, et immediatè a Radice ad Fructificationem ducit, vestitus Foliis, terminatus Fructificatione.

Folia transpirant, et adtrahunt (uti Pulmones in Animalibus), umbramque præbent.

Fulcra adminicula sunt, quibus licèt privata planta rarò perit.

Hybernaculum est Herbæ compendium super radicem (80), antequam excrescit.

82. TRUNCUS ( $8 \mathrm{I}$ ) Folia, et Fructificationem profert ; Species ejus sunt VI: Caulis , Culmus, Scapus, Pedunculus, Petiolus, Frons, Stipes; at Ramus pars est.

A. CAULIS, Truncus proprius herbæ, elevat Folia, Fructificationemque.

a. Simplex continuatâ serie versùs apicem extenditur.

I. Integer est simplicissimus, ramis vix ullis.)

2. Nudus Foliis destitutus : Euphorbia, Cactus, Stapelia, Ephedra, Cuscuta.

3. Foliatus foliis instructus est.

4. Flexuosus secundùm articulos horsum vorsum flexus : Ptelea.

5. Volubilis Tab. IV. f. I I5. spiraliter adscendens per ramum alienum.

Sinistrorsum \& secundùm solem vulgò: Humulus, Helsine, Lonicera, Tamus. Dextrorsum $\$$ contra motum solis vulgi: Convolvulus, Basella, Phaseolus, Cynanchum, Euphorbia, Eupatorium.

6. Reclinatus arcuatim versùs terram: Ficus.

7. Procumbens horizontaliter supra terram.

8. Repens Tab. IV. f. I I2. radiculas hinc inde exserens procumbendo: Heder , Bignonia.

$\mathrm{C}_{4}$ 
9. Sarmentosus Tab. VI. f. I3 I. repens, subnudus est. 10. Parasiticus alteri plantæ, nec terræ innatus: Epidendron, Viscum, Tillandsia.

I1. Teres cylindricus est.

12. Anceps angulos duos oppositos habet: Sisyrinchium.

13. Digonus, Trigonus, Tetragonus, Pentagonus, Polyg.nus, prxecdentis (I 2 ) species sunt.

14. Triquetrus latera tria plana obtinet.

15. Triangularis, Quadrangularis, Quinquangularis, Multangularis, ex numero angulorum prominentium.

6. Sulcatus sulcis excavatis latis profundis exaratus.

17. Striatus lineis tenuissimis excavatis inscriptus.

18. Glaber superficie lievi est.

9. Villosus pilis mollibus pubescens: Tomes, Rhus.

20. Scaber punctis eminentibus rigidiusculis exasperatus.

21. Hispidus setis rigidis adspersus.

$\boldsymbol{R}$ amosus est ramis lateralibus instructus.

22. Ascendens ramis sursum versis.

23. Diffusus ramis patentibus.

24. Distichus ramos situ horizontali exserit.

25. Brachiatus Tab. IV. f. II7. ramos decussatim oppositos habet.

26. Ramosissimus ramis multis absque ordine gravidus.

27. Fulcratus ramis descendens ad radicem: Ficus.

28. Prolifer ex apicis centro enittens tantùm ramos:

Pinus. Cetera ut in integro.

3. Compositus in ramulos subdivisus deliquescit adscendendo. 29. Dicbotomus Tab. IV. f. I I 6. bifariam semper divisus. 30. Subdivisus in ramos absque ordine.

3I. Articulatus internodiis geniculatus: Piper.

B. CULMUS, Truncus proprius Gramini ( 78$)$, elevat Folia, Fructificationemque: Species servat caulis quam plurimas. 32. Enodis, qui continuus est, nec articulis interceptus. 33. Articulatus Tab. IV. f. I I 4. variis articulis connectitur. 34. Squamosus Tab. IV. f. I I I. tectus squamis imbricatis. C. SCAPUS Tab. IV. f. I I 3. Truncus universalis elevans Fructificationem, nec Folia: Narcissus, Pyrola, Convallaria, Hyacintbus.

D. PEDUNCULUS Truncus partialis, elevans Fructificationem, nec Folia.

Pedicellus est Pedunculus partialis.

Determinatur Loco, aut Modo. 
Loco, quo planta inseritur basi.

I. Radicalis, qui e radice immediatè exit.

2. Caulinus, qui cauli insidet.

3. Rameus, qui e ramis exit.

4. Axillaris, ex ala, scilicet inter folium, et caulem; aut inter ramum, et caulem.

5. Terminxlis, qui ramos, vel caulem terminat.

6. Solitarius, qui unicus est in loco.

7. Sparsi, ubi plures absque ordine prognascuntur.

Modo, quo flores gerit, et connectit summitate.

8. Uniflorus, Biflorus, Triforus \&c. Multifiorus secundùm numerum fructificationum in singulo pedunculo.

9. Fasciculus colligit flores erectos, parallelos, fastigiatos, approximatos: Di.untbus barbatus.

10. Capitulum constat foribus plurimis in globum ferme congestis : Gomphrena.

11. Spica Tab. IX. f. 165. flores sessiles sparsim alterni in pedunculo communi simplici.

Secunda floribus ad unum, idemque latus versis.

Disticha floribus ad utrumque latus spectantibus.

12. Corymbus Tab. IX. f. I63. fit ex Spica, dum singuli flores pedunculis propriis instruuntur, situ elevato proportionali : Spirea Opuli folio, Ledum, Siliquose.

13. Panicula Tab. IX. f. $16 \%$ fructificatio sparsa in pedunculis diversè subdivisis.

Diffusa cùm divaricantur pedicelli.

Coarctata cùm pedicelli approximantur.

14. Thyrsus Panicula est coarctata in formam ovatam: Syringa, Petasites.

15. Racemus Tab. IX. f. I64. pedunculo ramis lateralibus brevibus constat: Vitis, Ribes.

16. Verticillus Tab. IX. f. I66. fit ex foribus numerosis subsessilibus, caulem annulatim ambientibus.

E. PETIOLUS, Trunci species, adnectens Folium, nec Fructificationem.

Petiolus, Pedunculus, Pediculus antecessoribus Synonyma fuere, nobis autem minimè.

Petiolus promit Folium, at Pedunculus Fructificationem; datur tamen rarissimè, qui utrumque, ut in Turnera, et Hibisco aliquo.

Caulis brachiatus semper caussat folia opposita. 
F. FRONS, Tab. III. f. r o8. Trunci species, ex Ramo coadunatus Folio, et sxpius Fructificatione.

Propria Filicibus, et Palmis.

G. STIPES basis Frondis est.

Proprius Palmis, Filicibus, Fungis.

83. FOLIUM (8I) consideratur secundùm Simplicitatem, Compositionem, aut Determinationem.

A. SIMPLEX (Tab. I.) est, cùm Petiolus gerit unicum Folium, et differt hoc Circumscriptione, Angulis, Margine, Superficie, Apice, Substantia.

*. Circumscriptio considerat peripheriam absque sinubus, et angulis.

I. Orbiculatum f. I. cujus diameter longitudinalis, et transversalis æquales, peripheria circinata.

2. Subrotundum f. 2. cujus figura orbiculato (I) proximè accedit.

3. Ovatum f. 3. cujus diameter longitudinalis superat transversalem, basi segmento circuli circumscripta, apice verò eodem angustiore.

4. Ovale s. Ellipticum f. 4. cujus diameter longitudinalis superat transversalem, superiore, et inferiore extremitate angustioribus.

5. Parabolicum Tab. III. f. I Io. cujus diameter longitudinalis superat transversalem, et a basi sursum angustatur in semiovatum.

6. Spatulatum Tab. III. f. I o9. cujus figura subrotunda, basi angustiore lineari elongata.

7. Cuneiforme Tab. I. f. 45 . cujus diamater longitudinalis superat transversalem, et sensim deorsum angustatur.

8. Oblongum f. 5 . cujus diameter longitudinalis aliquoties superat transversalem, et utraque extremitas segmento circuli angustior.

6. Anguli sunt partes prominentes folii horizontalis.

9. Lanceolatum f. 6. est oblongum (8) utrinque sensim versùs extremitatem attenuatum.

10. Lineare f. 7 . est æquali ubique latitudine, interdum utráque extremitate tantùm angustatur.

II. Acerosum f. 105. est lineare (10) persistens; ut in Pino, Abiete, Junipero, Taso. 
12. Subulatum f. 8. est inferiùs lineare $(10)$, at versùs apicem sensim attenuatur.

I 3. Triangulare f. 12. cum tres anguli prominentes ambiunt discum.

14. Quadrangulare, Quinquangulare f. 20. præcedentis $\mathrm{sp} \div$ cies sunt.

15. Deltoides f. 58. rhombeum est ex quatuor angulis, e quibus laterales minùs a basi distant, quàm reliqui.

16. Rotundum, quod angulis privatur.

c. Sinus discum folii in partes secant.

17. Reniforme f. 9. est subrotundum (2) basi excavatum, angulis destitutum,

18. Cordatum f. Io, est avatum (3) basi excavatum, desti:utum angulis posticis.

19. Lunulatum f. II. est s:ıbrotundum (2) basi excavatum, angulis posticis notatum.

20. Sagittatum f. 13. est triangulare ( 13 ) basi excavatum, angulis posticis instructum.

2I. Hastatum f. Is. est triangulare (13) basi lateribusque excavatis, angulis patulis: Rumex.

22. Panduroforme est oblongum (8) infernè latius, lateribus coarctatum.

23. Fissum f. I6. est divisum sinubus linearibus, marginibusque rectis.

Bifidum, Trifidum, Quadrifidum, Quinquefidum \&c. Multifidum, a numero hujus.

24. Lobatum f. 19. est divisum ad medium in partes distantes, marginibus convexis.

Bilobum, Trilsbum, f. I 7. Quadrilobum, Quinquelobum a numero hujus.

25. Palmatum f. 22. est longitudinaliter in partes plures subæquales divisum versùs basim, qua tamen cohæ.rent in unum,

26. Pinnatifidum f. 23. est transversim divisum laciniis horinzontalibus oblongis.

27. Lyratuin ' $\mathrm{a}$ b. II. f. 76 . est transversim divisum in lacinias, ita ut superiores majores sint, et inferiores remotiores,

28. Laciniatum Tab. J. f. 24. variè sectum in partes, partibus itid $m$ indeterminatè subdivisis.

29. Sinuatum f. 25. quod lateribus sinus dilatatos admittit. 30. Partitum f. 28. est divisum usque ad basim. 
Bipartitum, Tripartitun, Quxdrip.zrtitum, Quinquep.zrtitum, Multipartitum, a numero hujus.

3I. Integrum est indivisum, sinu omni destitutum; ergo hoc prioribus (17-30.) opponitur.

d. Apex est extremitas folii, in quem desinit. 32. Truncatum, quod lineâ transversali desinit.

33. Premorsum $\mathrm{f}$. 18 . quod obtusissimum terminatur incisuris inæqualibus.

34. Retusum f. 46. quod terminatur sinu obtuso.

35. Emarginatum f. 45 . quod terminatur crenâ.

36. Obtusum f. 40. quod termianatur quasi intra segmentum circuli.

37. Acutuin f. 4r. quod terminatur angulo acuto. 38. Acuminatum f. 42 . quod terminatur apice subulato. Obtusum acumine f. 43 . patet ex dictis $(36.38)$. 39. Cirrhosum, quod terminatur cirrho Tab. II. f. 72. ( $\left(9.8_{4}\right)$ Gloriosa, Flagellaria, Nissolia $T$.

e. Margo est extrema ora fulii ad latera, intacto disco folii. 40. Spinosum, quod margine exit in acumina duriora, rigida, pungentia.

Inerme spinoso folio opponitur.

4r. Dentatum Tab. I. f. 30. quod acumina horizontalia, folii consistentiâ, spatio remota habet.

42. Serratum f. 3 r. quod angulis acutis imbricatis extremitatem respicientibus notatur.

43. Crenatum f. 38. cujus margo angulis neutram extremitatem respicientibus secatur.

Obtusé f. 36 . acuté f. 35 . duplicaté f. 33 .

44. Repandum f. 29. cujus margo angulis, eisque interjectis sinubus, circuli segmento inscriptis, terminatur.

45. Cartilagineum f. 34 . cujus margo cartilagine, a substantia folii diversissima, firmatur.

46. Ciliatun f. 50. cujus margo setis parallelis longitudinaliter obvallatur.

47. Lacerum, quod margine variè sectum est segmentis difformibus.

48. Erosum f. 2 I. cùm folium sinuatum (29) margine sinus alios minimos obtusos acquirit.

49. Integerrimum f. 42 . cujus margo extimus integer absque omni crenâ est.

f. Superficies discum folii Supinum (superiorem), vel Pronum (inferiorem) tegit. 
50. Viscidum, quod humore non fluido, sed tenaci oblinitum.

5x. Tomentosum f. 48. villis intertextis vix conspicuis tegitur, ergo sæpius albidum; uti Plantæ marinæ, et campestres ventis expositæ.

52. Lanatum quasi telâ a raneæ inductum: Salvia, Sideritis.

53. Pilosum f. 47. cùm pili distincti elongati tegunt superficiem: Cortusa.

54. Hispidurn f. 49. cùm setæ rigidiusculæ fragiles per discum folii sparsæ sunt.

55. Scabrum; cùm tubercula rigidiuscula per discum folii sparguntur.

56. Aculeatum, cùm acumina pungentia rigida occupant discum.

57. Striatum, cùm lineæ superficiales excavatæ longitudinales parallelæ inscriptre sunt.

58. Papillosum f. 54. quod tegitur punctis vesicularibus. 59. Punctatum, quod punctis excavatis adspersum est.

6o. Nitidum, quod glabritie lucidum est: Ferula canadensis, Angelica canadensis.

6I. Plicatum f. 37 . cùm discus folii versùs marginem ad angulos ascendit, et descendit: Alchemilla.

62. Undulatum fit, cùm discus folii versùs marginem convexè adscendit, et descendit.

63. Crispum f. 39 . cùm peripheria folii major evadit, quàm discus admittit, ut undulatum fiat. Folia omnia Crispa monstra sunt.

64. Rugosum f. $5 \mathrm{I}$. cùm venæe foliorum contractiores evadunt, quàm discus, ut interjecta substantia ascendat: Salvia.

65. Concavum, cùm margo folii arctior fit, quàm ut discum circumscribat, unde deprimitur discus.

66. Venosum f. 52 . cùm vasa discurrentia evadunt ramosissima, et anastomoses nudo oculo exhibent.

67. Nervosum f. 53. cùm vasa simplicissima absque ramulis extenduntur a basi versùs apicem.

68. Coloratum, quod alium colorem, quàm viridem induit: Anaranthus tricolor.

69. Glabrum, quod superficie lævi est absque omni inæ qualitate.

g. Substantia folii consideratur secundùm latera.

70. Teres f. 62. quod maxima ex parte cylindricum est. 
Semicylindraceum est teres, sed altero latere longitudinaliter planum.

7r. Tubulosum, quod internè (si detruncetur) cavitatem habet: Cepa.

72. Carnosum, quod internè pulpa repletum est: Succulentie.

73. Compressum, quod a lateribus marginalibus oppositis comprimitur, ut substantia folii major fiat, quàm discus. 74. Planum, quod utramque superficiem ubique parallelam gerit.

75. Gibbum, quod utramque superficiem facit convexam $(76)$, mediante copiosiore pulpa.

76. Convexum, quod in disco magis elevatum est.

77. Depressum, quod in disco magis deprimitur, quàm ad latera.

78. Canaliculatum f. 6r. ex sulco profundo, secundùm totam longitudinem, excavatum in dimidiatum ferè cylindrum.

79. Ensiforme est anceps Tab. III. (83), a basi versùs apicem attenuatum.

8o. Acinaciforme Tab. I. f. 56. est compressum carnosum, altero margine convexo angusto, altero rectiore crassiore: Mesembryanthemum Dill.

8I. Dol.abriforme f. 57. est compressum, subrotundum, obtusum, extrorsum gibbum (75) acie acuta, infernè teretiusculum: Mesembryanthemum Dill.

82. Linguiforme f. 55. est lineare carnosum, obtusum, subtus convexum, margine sæpius cartilagineo: $M e-$ sembryanthemum Dill.

83. Anceps est, quod duos angulos prominentes longitudinales disco convexiore admittit.

84. Triquetrum f. 59. cujus tria latera longitudinalia plana sunt in folio subulato.

85. Sulcatum f. 6o. quod angulis numerosis, totidemque interjectis sinubus, longitudinaliter exaratur.

86. Carinatsm, si pars prona disci prominet longitudinaliter.

87. Membranaceum, quod inter utramque superficiem nullâ evidenti pulpâ scatet. 
B. COMPOSITUM (Tab. II.) respicit folia plura, in uno petiolo, secundùm Structuram, aut Gradus.

h. Structura considerat foliorum insertionem.

88. Compositum, cùm Petiolus simplex profert plus quàm folium unicum.

89. Articulatum Tab. III. f. 100. cùm folium unum ex alterius apice excrescit.

90. Digitatum, Tab. II. f. 66. cùm Petiolus simplex apice adnectit Foliola plura.

91. Binatum Tab. II. f. 63. est digitatum (89), foliolis duobus.

92. Ternatum f. 64.65. est digitatum (89), foliolis tribus. 93. Quinatum est digitatum Tab.II.f. 66. sed foliolis quinque. 94. Pinnatum, cùm Petiolus simplex lateribus adnectit Foliola plura.

cum impari f. 68. est pinnatum terminatum foliolo impari.

cirrbosum f. 72. est pinnatum terminatum cirrho $(\$ .84)$

abruptum f. 69. est pinnatum terminatum neque cirrho, neque foliolo.

opposite', est pinnatum, foliolis oppositis (I I I ).

alternation f. 70 . est pinnatum, foliolis alternis ( I I 2 ). interrupte f. $7 \mathrm{I}$. pinnatum, foliolis alternis minoribus.

articulate f. 75 . pinnatum - petiolo communi articulato.

decursive f. 74. pinnatum foliolis decurrentibus ( I 2 I) per Petiolum.

95. Conjugatum est, cùm pinnatum (94) constat modò foliolis duobus, nec pluribus.

i. Gradus considerant subdivisionem Petioli communis. 96. Decompositum, cùm Petiolus semel divisus adnectit foliola plura.

97. Bigeminatum, cùm Petiolus dichotomus $(\$ .82)$ apicibus adnectit foliola quatuor.

98. Biternatum (Dup!icato-ternatum) f. 77. cùm Petiolus affigit tria foliola ternata: Epimedium.

99. Bi-pinnatum (Duplicato-pinnatum) f. 78. cùm Petiolus lateribus affigit foliola pinnata (94). 
roo. Pedatum (Ramosum) f.67. cùm Petiolus bifidus latere tantùm interiori adnectit foliola: Passiflora, Arum. Ior. Supradecompositum cùm Petiolus aliquoties divisus adnectit plurima foliola.

102. Triternatum (Triplicato-ternatum) f. 79. cùm Petiolus affigit tria foliola Biternata (98).

103. Tripinnatum (Triplicato-pinnatum) f.80. cùm Petiolus affigit plura foliola Bipinnata (99).

C. DETERMINATIO (Tab. III.) aliunde (nec a propria structura) notam adquirit; uti a Loco, Situ, Insertione, aut Directione.

k. Locus, quo adnectuntur parti plantæ.

104. Seminale Tab. III. f. 88. quod antea Cotyledon (\$.86: VI.) fuit, et primum in planta est.

ro5. Radicale, quod Radici (\$.80) insidet.

ro6. Caulinuin, f. 89. quod Cauli (\$.82, A) insidet.

107. Rameum, f. 90. quod Ramo (82) insidet.

108. Axillare (Subalare), quod ad rami (82) exitum inseritur.

ro9. Florale f. 9 r. quod ad Floris $(82, C)$ exortum inseritur.

1. Situs est dispositio Foliorum in Plantæe caule.

I ro. Stellata (Verticillata) f. ror.cùm folia plura, quàm duo verticillatim caulem ambiunt.

- i I. Terna, Quaterna, Quina, Sena. \&c. f. 102. sunt species numeri Stellatorum ( I 10$)$ : Nerium, Brabejum, Hippuris.

II 2. Opposita f. 82-87. 103 ; cùm caulina folia duo, per paria decussatim, e regione collocantur.

I 3. Alterna f. I04. cùm unum post alterum tanquam per gradus exit.

I14. Sparsa, cùm in planta sine ordine copiosissima.

I 5 . Conferta f. I05. cùm ita copiosa, ut ramos occupent totos, vix relicto spatio.

I 16. Imbricata f. I06. si conferta, et erecta (I I5), ut invicem se quoad partem tegant.

Ir 7. Fasciculata $f$. 107. si ex eodem puncto plura folia prodeunt: Larix.

II 8. Disticha, si omnia folia duo latera rami tantùm respiciunt: Abies, Lonicera, Diervilla.

$m$. Insertio folii fit basi ejusdem. 
14. Yeltatum f. 92. si Yetiolus (non margini, seu basi) disco folii inseritur: Nymphea, Hernandia, Colocasia.

120. Petiolatum f. 93. si Petiolus (\$. 82.) adnectitur folio ad marginem baseos.

I2 I. Sessile f. 94. si Folium petiolo (\$.82. E.) careat, et immediatè cauli ( 82. A) adnectatur.

22 Decurrens f. 95 . si basis foliisessilis ( 2 I ) dein ultra basin extendatur deorsum per caulem : Verbesina, Carduus, Sphoranthus.

223. Amplexicaule f. 96 . si basis folii undique ambiat latera caulis transversim.

Semiamplexicaule a priori gradu minore differt.

24. Perfoliatum f. 97 . si basis folii undique cingat transversim caulem : Bupleurum.

I25. Connaium f. 98. si folia opposita ( 1 I 2) inter se connata in unum: Lonicera, Eupatorium.

I26. Vaginans f. 99. si folii basis format tubum cylindrin. Directio. cum, ramum ( 82 ) vestientem: Polygonum, Rumcs.

27. Adversum, quod latus (non cælo, sed) meridiei obvertit: Amomum.

I28. Obliquum, cùm basis folii cælum, apex horizontem spectat: Protea, Fritillaria.

I 29. Inflexum (Incurvum) f. 82. dum sursum arcuatur versùs caulein.

I30. Adpressum, dum discus folii approximatur cauli.

I 3 I. Erectum f. 83. quod ad angulum acutissimum cauli adsidet.

I32. Patens f. 84. quod ad angulum acutum cauli insidet.

133. Horizontale f. 85 . quod ad angulum rectum a caule discedit.

I34. Reclinatum f. 86. quod deorsum curvatur, ut apex fiat basi inferior; quibusdam etiam $R$ sfexum dicitur.

I35. Revolutum f. 87 . quod deorsum revolvitur.

I36. Dependens, quod rectâ terram spectat.

137. Radicans, si folium radices agat.

I 38. Natans, quod superficiei aqua innatat: Nymphea, Potamogeton.

I39. Demersum, quod infra aquæ superficiem absconditur. 
84. FULCRA (8I) adminicula plantæ sunt, pru commodiore sustentatione; numerantur hodie VII: Stipula, Bractea, Spina, Aculeus, Cirrbus, Glandula, Pilus.

r. STIPULA Tab. V. f. I 8 . b. est squama, qur basi Petiolorum (82: V), aut Pedunculorum ( 82 :IV) enascentium utrinque adstat: Papilionacee, Tamarindus, Cassia, Rosa, Meliantbus, Liriodendrum, Armeniaca, Persica, $\mathrm{Pa}$ dus \&c.

2. $D R A C T E$ A fig. 1 20. a. dicitur Folium forale (83: 109), cùm colore, et figura recedit a ceteris : Tilia, Fumaria. bulbosa, Lavandula Stochas, Salvia Horminum.

3. SPINA. f. I I I. a. b. est mucro plantæ e ligno plantæ. protrusus: Prunus, Rbamnus, Hippophä̈, Celastrus, $L y$ cium.

Culturâ sæpius evanescit : ut in $P$ yro.

4. ACULEUS f. I $22, \mathrm{I} 23 \cdot \mathrm{a}$. b. est mucro plantx, ejusdem cortici tantùm affixus: Rosa, Rubus, Ribes, Berberis.

5. CIRRHUS Tab. V. f. I I8. a. est vinculum filiforme spirale, quo planta alio corpori alligatur: Vitis, Bannisteria, Cardiospermum, Pisum, Bignonia.

6. GLANDULA Tab. V.f. II9. a. f. i I8. est Papilla humorem excernens: Urena, Ricinus, Fatrcpha, Passiflora, Cassia, Opulis, Turnera, Salix tetrandra, Heliocarpus, Bryonia zeyl. Acacia cornigera, Baubinia aculeata, Armeniaca, Amygdulus, Morisona.

Locus : Petioli, Foliorum Serraturæ, et Stipulæ. te-nellæ.

7. PILUS est ductus excretorius plantæ setaceus.

Guettardus. in his primaria detexit.

85. HYBERNACULUM (8I) est pars Plantæ includens Herbam ( $8 \mathrm{I}$ ) embryonem ab externis injuriis; estque Bulbus, vel Gemma.

x. BULBUS est hybernaculum caudici descendenti (80) insidens.

Squamosus Tab.VI, f. 125 . constans imbricatis lamellis: Liliuim.

Solidus f. I 26. constans substantiâ solidâ : Tulipa.

Tunicatus f. I 27. constat tunicis numerosis: Cepa officinarum. 
Articulatus constans lamellis catenulatis: Lathrea, Martynia, Adoxa.

2. GEMIMA est hybernaculum caudici adscendenti Y 80 ) insidens.

Constat vel Stipulis, vel Petiolis, vel Foliorum rudimentis, vel Squamis corticalibus.

Uti Plantæ pleræque in frigidis, sic in calidis terris vix ullæ, gemmis gaudent.

Caren Gemmis Arbores varix: Philadelphus, Frangula T. Alaternus T. Paliurus T. Fatropha, Hibiscus, Babobab, Fusticia, Cassia, Miimosa, Gleditsia, Erythrina, Anagyris, Medicago, Nerium, Viburnum, Rhus, Tamarix, Hedera, Erica, Malpighia, Lavatera, Solanum, Asclepias, Ruta, Geranium, Petiveria, Pereskia Pl. Cupressus, Thuja, Sabina.

Species Gemmarum varix sunt.

Decidue in Dentaria, Ornithogalo, Lilio, Saxifragâ. Foliiferce, non floriferce: Alnus.

Foliifere, et floriferce distincte: Populus, Salicis species,

Fraxinus.

Foliiferce, et floriferce feminece: Corylus, Carpinus.

Foliifere, et floriferce masculce: Pinus, Abies.

Foliifere, et floriferce hermaphrodite: Daphne, Ulmus,

Cornus, Amygdalus.

Foliifero-florifere, ut pleræque arbores.

Laflingii Diss. de Gemmis arborum cetera docet. 


\section{FR UCT IF I CA TIO.}

86. FRUCTIFICATIO (79) Vegetabilium pars temporaria, Generationi dicata, antiquum terminans, novum incipiens; hujus Partes VII. numerantur.

1. CALYX, Cortex plantæ in Fructificatione præsens. 1. Perianthium, Calyx plantæ (78) Fructificationi contiguus.

a. Fructificationis, Stamina Germenque includens.

b. Floris, Stamina absque Germine continens.

c. Frutus, Germen absque Staminibus continens.

2. Involucrum T. VII. f. I 35. Calyx Umbellæ (29) a flore remotus.

a. Universale Umbellæ universali subjectum. c. c.

b. Partiale umbellulæ partiali subjectum. d. d.

3. Amentum Tab. VII. f. I 37: Calyx ex Receptaculo communi paleaceo-gemmaceo.

4. Spatha f. I 32. I33. Calyx Spadicis (3 I) longitudinaliter ruptus.

5. Gluma f. I 34. Calyx Graminis valvis amplexantibus. Fig.a. Arista, mucro glumæ insidens. Tab. VII. I 34. b. b.

6. Calyptra f. I 36. b. c. Calyx Musci cucullatus, antheræa. superimpositus.

7. Volva f. I 39. Calyx Fungi membranaceus, undique lacerus.

II. COROLLA, Liber plantæ in Flore præsens.

Petalum, tegmen floris corollaceum.

a. Tubus Tab.VII. f. I 42. a. corollæ monopetalæ pars inferior tubulosa.

6. Limbus f. I42. b. corollæ monopetalæ pars supes rior dilatata.

Campanulatus, ventricosus absque tubo.

Infundibuliformis, conicus, tubo impositus.

Hypocrateriformis f. 142. planus, tubo impositus.

Rotatus, planus, nulli tubo impositus.

Ringens, irregularis, in duo labia personatus.

c. Unguis f. I 44. a. Corollæ polypetalæ pars inferior basi affixa.

d. Lamina f. I i 4. b. Corollæ polypetalæ pars superior patula. 
Cruciformis $\mathrm{f}$. 144. petalis quatuor æqualibus patens. Papilionacea irregularis: petalo inferiore cymbiformi, superiori adscendente; lateralibus solitariis.

9. Nectarium pars mellifera flori propria f. I45. I46. I 47.148 .2 .

III. STAMEN, Viscus pro Pollinis præparatione.

ro. Filamentum, pars elevans, adnectensque Antheram. Tab. VIII. f. I 49 . e. e. f. I 50. a.

II. Anthera, pars floris gravida.Polline, quod matura dimittit, f. f. f. I so. b.

12. Pollen, pulvis Floris, humore rumpendus, atomosque elasticos ejaculans, f. I 5. a. b.

IV. PISTILUM, Viscus fructui adhærens, pro Pollinis receptione Tab. VIII.

13. Germen, rudimentum Fructûs immaturi in flore $f$. I 49. g. f. I 52 . a.

14. Stylus, pars Pistilli, Stigma elevans a Germine f. 149.c. f. I52. b.

15. Stigma, summitas Pistilli madida humore Pollen rumpendo, f. I 49. d. f. I 52. c. c.

V. PERICARPIUM, Viscus gravidum seminibus, qux matura dimittit.

16. Capsula Tab. VIII. f. I60. 159. a. 161. Pericarpium cavum, determinatè dehiscens.

Valvula f. I 59.a. paries, quo fructus tegitur externè.

Dissepimentum f. I6o. b. paries, quo fructus internè distinguitur in concamerationes plures.

Columella f. I60. c. pars connectens parietes internos cum seminibus.

Loculamentum f. $\mathbf{x} 60$. d. concameratio vacua pro seminum loco.

17. Siliqua f. I 55. a. b. c. Pericarpium bivalve, affigens semina secundùm suturam utramque.

18. Legumen f. is 4. a. a. Pericarpium bivalve, affigens semina secundùm suturam alteram tantùm.

19. Conceptaculum f. I 53. Pericarpium univalve, latere longitudinaliter dehiscens, a seminibus distinctum.

20. Drupa f. 157. a. Pericarpium farctum evalve, Nucem b. b. continens.

21. Pomum f. 156. a. Pericarpium farctum evalve, $\mathrm{Ca}-$ psulam b. continens. 
22. Bacca f. I58. Pericarpium farctum evalve, Seminx ceteroquin nuda continens.

23. Strobilus Tab. VII. f. I 38. Pericarpium ex Amento factum.

VI. SEMIEN, pars vegetabilis decidua, novi rudimentum, Pollinis irrigatione vivificatum.

24. Semen (propriè), novum vegetabilis rudimentum, humore rigatum, vesicâ tunicatum.

a. Corculum, novæ plantæ primordium intra semen. Pluinula pars corculi squamosa adscendens.

Rostellum pars corculi simplex descendens.

b. Cotyledon, corpus laterale seminis, bibulum, caducum.

c. Hilum, cicatrix externa seminis ab ejusdem affixione in fructu.

d. Arillus, tunica propria exterior seminis, sponte secedens.

e. Coronula, calyculus adharens, quo volitat.

Pappus Tab. VIII. f. 162 . corona pennacea, pilosave volitans a. b. Sitpes, filum elevans, connectensque Pappum, et semen. d. c.

f. Ala, membrana, qua volitante disseminatur, affixa. semini.

25. Nux, semen tectum epidermide osseâ.

26. Propago, semen II usci decorticatum, detectum 1750 . VII. RECEPTACULUM, basis, qua partes fructificationis. VI. connectuntur. Tab. VII.

27. Receptaculum Proprium, quod partes unius tantùm fructificationis respicit.

Fructificationis, pro flore, et fructu commune. Floris, basis, ubifloris partes absque Germine affiguntur. Fructûs, basis pro fructu, remota a floris receptaculo. Seminum, basis, qua semina intra Pericarpium aftiguntur. Tab. VIII. f. I6I.

28. Receptaculum Commune connectens flosculos plures ita, ut si aliqui eximantur, irregularitas caussetur f. I 40. a. Palea, lamella innata flosculos distinguens. f. I4I. b.

29. Umbella, receptaculum ex centro eodem elongatum in pedunculos filiformes proportionatos. Tab. VII. f. I 35 . a. Simplex, quæ non subdividitur, ut Panax.

b. Composita, cujus omnes pedunculi apice umbellulas gerunt. 
Universalis, quæ alias umbellulas apicibus gignit: aa.

Partialis umbellula, quæ prodiit ex universali. b. b. 30. Cyma, receptaculum ex centro eodem universali, partialibus verò vagis, elongatum in pedunculos fastigians. 3I. Spadix Tab. VII. f. I 33. b. receptaculum Palma enatum intra spatham, in ramulos fructificantes.

87. Partes FLORIS: Calyx, Corolla, Stamen, Pistillum. FRUCTUS: Pericarpiurn, Semen, Receptaculum. FRUCTIFICATIONIS itaque Flos, Fructus sunt.

FLORIS Definitio ab auctoribus traditur variè:

fungii: Fios est pars plantæ tenerior, colore, et figurä, vel utroque insignis, rudimento fructûs cohærens.

$\boldsymbol{R} a j i:$ Flos est pars plantæe tenuior, fugax, colore, et figurâ, vel utroque insignis, fructui pravia, eique plurimum cohærens, et tenello tegendo, fovendoque inserviens, quæ, postquam explicatur, brevi aut discedit, aut marcescit.

Tournefortii: Flos est pars plantæ, formâ, et naturâ ceteris dissimilis, fructui nascenti plerumque adhærens, cui primum alimentum ad tenerrimas ejus partes explicandas ministrare videtur.

Pontedere: Flos est pars plantæe, formâ, et naturâ ceteris dissimilis, embryoni semper, si tubâ instructus est flos, vel adhærens, vel quàm proximè affixa, cujus usui inservit; si verò tuba careat, nulli embryoni adhærens. Ludrvigii: Flos est pars plantæ filamentosa, et membranosa, quæ a reliquis loborum elegantiâ, et subtiliore fabricẩ in vulgus distinguitur.

FRUCTUS Definitio:

Fungii: Fructus pars plantæ annua, flori cohærens, et succedens, qui, ubi ad perfectionem pervenerit, sponte a planta abscedit, et commodâ nutrice exceptus novæ plantæ facit initium.

Calycem esse partem floris, quamvis sæpius in fructu adsit, inde liquet, quod caly $\mathbf{x}$ nunquam erumpat in flore post florescentiam.

Patagonulce calyx in fructu maximè ampliatur.

Deciduis calycibus multi flores instruuntur, qui cadunt ad primam floris explicationem, ut in Epimedio, $P_{a-}$ pavere. 
88. Essentia FLORIS (87) in Antbera (36), et Stigmate (86) consistit.

FRUCTUS (87) in Semine (86).

FRUCTIFICATIONIS (87) in Flore, et Fructu. VEGETABILIUM ( $\left.7^{8}\right)$ in Fructificatione (87).

Character partium plantarum difficilè eruitur, nisi assumantur duo prima Pollinis, et Seminis.

ฐ. POLLEN est pulvis vegetabilium (\$.3.), appropriato. liquore madefactus rumpendus, et substantiam sensibus nudis imperscrutabilem elasticè explodens.

2. SEMEN est pars plantæ decidua, rudimento novæ plantæ foeta, et polline vivificata.

3. ANTHERA est vas Pollen (I) producens, et dimittens. 4. PERICARPIUM est vasculum Semina (2) producens, dimittensque.

5. FILAMENTUM est pes Anthere (3), quo vegetabili alligatur.

6. GERMEN est Pericarpii (4), Seminisve (2) rudimentum immaturum, existens præcipuè eodem tempore, quo Anthera (3) Pollen (I) dimittit.

7. STIGMA est apex Germinis (6) roridus.

8. STYLUS est pes Stigmatis (7), connectens illud cum Germine (6).

9. COROLLA, et CALYX sunt tegumenta Staminum (1.3. 5.), et Pistillorum (6. 7. 8.), quorum hic ex Epidermide corticali, illa ex Libro orta est.

10. RECEPTACULUM est, quod connectit partes præ$\operatorname{dictas}(5.6 .9)$.

I I. FLOS ex Anthera (3), et Stigmate (7) nascitur, sive tegumenta (9) adsint, sive non.

12. FRUCTUS ex Semine (2), sive pericarpio (4), sive non tectum, dignoscitur.

13. FRUCTIFICATIO omnis gaudet Antbra (3), Stigmate (7), et Semine (2).

14. VEGETABILE omne Flore (I I), et Fructu (I 2 ) instruitur; ut nulla species his destituta.

Seminis essentia consistit in Corculo $(\$ .86)$, quod Cotyledoni adnectitur, et ab eodem involvitur, dum tunicâ propriâ arctè vestitur.

Corculi essentia consistit in Plumulâ, quæ Punctum vitæ ipsius plantæ est sub minimâ mole, et uti Gemma cre- 
scit in infinitum; at basis Plumula est Rostellum, qua descendit, et Radices agit, olim matri contigua.

Propagines Muscorum sunt semina destituta tunicâ, et cotyledonibus, adeoque nudi corculi Plumula, ubi Rostellum infigitur calyci plantæ.

89. PERIANTHIUM (86: r.) a Bractea (84) differt, quòd illud, maturo fructu, si non priùs, marcescat; Foliola floralia non item.

Bractere exempla petenda e Mrelampyro, Monarda, Salvia, $L a-$ vandula, Bartsia, Hebenstretia, Mussenda, Tilia, Fumaria.

Bracteam pro Perianthio sæpius, et facilè assumi, patet ex Helleboro, Nigella, Passifiora, Hepatica, Pegano.

Perianthii definitio apud Auctores:

Malpigh. Calyx floris basis est, et fulcimentum, suâ cor. poraturâ foliorum stamina fovet, quia et ipsorum progressum plerumque cooperit.

Raji: Calyx est, qui florem sustentat, ejusque velut basis, et fundamentum; est ideoque crassior, et minùs insignis flore.

Tournefort: Caly $\mathrm{x}$ dici debet pars floris posterior, crassitudine quadam notabili a pediculo distincta.

Luidwig : Calyx, seu Perianthium est membrana exterior floris.

90. COROLLA (86: IV.) a PERIANTHIO (86: I.) distinguitur, quòd illa cum Staminibus (86: III.) situ alternat, Periantbium autem opponitur.

Tournef. Petala sunt folia illa, quæ formâ, et colore plerumque ceteris partibus præstant \& quæque nunquam fiunt seminis involucrum.

Columna, Petalum esse Floris Folium, primus dixit.

Stamina alternare cum Petalis, uti Petala cum Perianthio, adeoque et Stamina opponi laciniis calycinis, patet ex Tetrandris, et Pentandris completis.

Exempla regulæe petas ex Chenopodio, Urtica, Parictaria, ubi Corolla deest.

Statuere, ubi ex Perianthio, et Corollâ alterum adest, esse hoc Corollam, uti pars præstantior, negant Ammania, Isnarda, Peplis, Ruellia, Campanula, quæ sæpe corollas excludunt, non verò calyces. 
Calycem, uti a cortice plantre ortum, magis rudem, crassumque esse, quàm Corolla, e tenero, colorato, molli Libro producta, cliique patet: Limites autem horum determinantur vix unquam, nisi a colore, qui non sufficiens est, ut in Barisia.

Plurimi flores ferunt Corollas coloratas, nudas, caducas, sub statu florescentiæe, quæ dein indurantur, virescunt, persistunt, e. gr. Helleborus, Ornitbogalum.

Limites inter Calycem, et Coroilam absolutos, naturam non posuisse, patet ex $D a p b n i d e$, ubi connata ambo, et margine omnino unita, veluti folium Buxi. cfr. Class. plant.5.n. I I.

Euphorbiam monopetalams statuunt varii, qui calycem pro corolla habent: paltas autem Floris esse Petala, docent species annuæ indicæ petalis albis distinctissimis.

91. PETALORUMi (86: 8.) Numerus a basi Corollæ: Laciniarum autem a medio Limbi, aut Laminæ desumendus est.

Rivini : Tot Petala numeramus, in quet resolvitur Flos deciduus.

Monopetali: Trientalis, Oxalis, Ledum, Anagallis, Veronica. Pentapetali: Alsines.

Tetrapetalus Oxycoccus perperam habetur, licet in 4. partes resolvatur, nam antea unum erant.

Rivini: Quicunque flores supra fructum hærent fundo integro, monopetali sunt, etiamsi tales non decidant. Quicunque in sinu suo fructum fovent, petalisque variis cohærentibus constant, contrariò, secundùm numerum petalorum habendi, quamvis non defluant.

Polypetalorum numerus ab ordinibus insertionum determinatur, ut in Hepatica.

Grecè enuntiatur vulgò Petalorum numerus:

Apetalus, Petalodes, Monopetalus, Dipetalus, Tripetalus,

Tetrapetalus, Pentapetalus, Hexapetalus, Polypetalus.

Segmenta corollæ monopetalæ a variis in oides terminantur:

Pentapetaloides, Tetrapetaloides.

Periantbium difficiliùs distinguitur in monophyllum, et polypbyllum.

Vaillantii: Si Calyx embryonem coronat, est monophyllus, unumque cum embryonibus corpus constituit.

Calyx monophyllus est, quoties monopetalus flos est. perperam 
92. STRUCTURAM triplicem Fructificationis, in omnibus ejusdem partibus (86), ubique observat Botanicus: Naturalissimam, Differentem, et Singularem; Et has secundùn quatuor Dimensiones: Numerum, Figuram, Proportionem, et Situm adtento oculo describat.

Dimensionis 4. requisita: Numerus, Figura, Proportio, Situs, sunt totidem artis columnx.

Figura deterninatur terminis foliorum. \$. 83 .

Proportio æstimatur secundùm altitudinem partium inter se. Situs, s. Insertio, aut comexio partium eædem sunt. Lubrica sæpius sunt: Magnitudo, Color, Odor, Sapor. 93. NATURALISSIMA Structura (92) Fructificationis a pluralitate existentium desumitur: in a. Numero (94), B. Figura (95), y. Proportione (96), 8. Situ (97).

Naturalissima in plerisque Plantis occurrit:

Calycem crassiorem, brevicrem Corollầ tenerâ caducâ. Pistillum in medio floris intra Stamina; Antheras insidere

Filamentis, et Stigmata Stylis Ẻc. communiter obtinet. Differunt omnes fructificationes, et sic etiam conveniunt omnes.

Naturalissima structura ex usu adsuescit, nec observatur dein a Botanico genuino; hanc diffusè describunt idiotæ, et peregrinatorum turba, eoque produnt ignorantiam, quam perversi extollunt.

Agrostographus eximius hîc parùm impegisse mihi videtur, alioquin verè Magnus.

Exemplo Caracterem barbarè constructum proponam: $\mathrm{C}_{\mathrm{A}}$ LYX viridis, erectus, brevis, quinquepartitus, segmentis angustis, angustioribus quàm longis. Corotra petalis quinque, explanatis, basi angustioribus, coloratis, tenuissimis, patentibus, insertis receptaculo intra calycem, defluentibus ad basim, alternantibus cun calycis laciniis, cadentibus ante maturitatem fructûs. STAMinuar Filamenta nonnulla, angusta, subteretia, supernè angustiora, inserta receptaculo intra corollam, alternata cum petalis, sed calyci opposita. Anthera unica in singulo flamento, quæ rumpitur, redditque farinam, et contabescit. Pistillum centrum floris occupat intra stamina, 
habet pro basi Rudimentum fructûs parvum, cujus apici styli insident, germine angustiores, qui nec plani sunt, sed angusti, distincti a corolla, et stigmata gerunt in apicibus crassiuscula. Postquam defloruit Planta, excrescit germen, et fit Pericarpium, quod replet calycem, fit durum, et a pice vestigium styli gerit, tandem rumpitur in varia loculamenta, et valvulas, ejicitque semina acuminata, quæ aftixa fuere lateribus pericarpii internis. $Q$ uis diceret LINUM esse?

94. NUMERUS naturalissimus (93) est, quod Caly $x$ in tot segmenta, quot Corolla, dividitur, quibus Filamenta respondent, singulo singulis Fniberis instructo. Pistilli autem divisio cum Pericarpii loculis, aut Seminum Receptaculis convenire solet.

Quinarius numerus in Fructificatione maximè frequens est; ut ex Pentandris, Syngenesistis, aliisque patet.

Calyx, et Corolla quinquefida in plurimis evadunt.

Exempla pro numero naturalissimo sint Lysimacbia, Linum. 95. FIGURA naturalissima (93) est, quod Caly $x$ minùs patens contineat Corollam sensim dilatatam, Staninibus, et $P$ istillis erectis, sensim attenuatis instructam; hisce, excepto Calyce, decidentibus, Pericarpium intumescit, et extenditur, Seminibus refertum.

Calyx Periantbium erectius est, ut fulciat corollam.

Corolla magis, vel minùs ad infundibuliformem sæepius accedit. Staminum Filamenta, sursum plerumque attenuata, subulata, erecta, parùm apicibus reflexa.

Pistillorum Styli in plerisque erecti,et angusti, uti filamenta. Pericarpium intumescere, et accrescere vulgatissimum est.

96. PROPORTIO naturalissima (93) profert Calycem Corollâ minorem, cum Staminibus, et Pistillis longitudine æqualibus, si flos erectus est.

Nutans flos gerit Pistillum Staminibus longius.

Decumbens Stamina, Pistillaque ad latus inferius declinata.

Adscendens verò Stamina, Pistillaque sub latere superiore recondita. 
Calyx brevior, quum gemma fructificationis sit. Corolla magna in plerisque floribus evadit. Stamina, et Pistilla, vix calycem exsuperant in multis. Nutans flos, ut faciliùs foecundetur. $\$$. I 45 .

Fritillaria, Campanula, Galantbus, Geranium:

hinc non ex proprio pondere.

Decumbens, in Cassia, Diadelpbia omni. Adscendens, in Didynamia gymnosperma.

Pistillum ubi Staminibus brevius, connivent Antheræ: $S_{a}$ xifraga, Parnassia.

97. SITUS naturalissimus (93) est, quod Perianthium involvat Receptaculum, cui Corolla alternatim adnascitur; huic autem interiùs respondent Filamenta, quorum apicibus Antberce incumbunt. Centrum Receptaculi occupat Germen, cujus apici Stylus insidet, summo Stigma gerens. Hisce decidentibus Germen in Pericarpium excrescit, calyce sustentatum, includens Semina adnexa fructûs receptaculo. Receptaculum Floris frequentiùs subnascitur, rariùs circumnascitur, vel supernascitur.

Series Fructificationis hæc est, quod Calyx extra Corollam, intra Corollam verò Stamina, sed hæc extra Pistilla collocentur: Exceptio rarior. \$. I I r.

Receptaculum connectit Calycem, Corollam, et Stamina modis quatuor.

a. Receptaculum ad basim germinis; ut in plerisque.

Pistillum abiens in fructum. Tournef.

b. Receptaculum ad apicem germinis; in multis.

Calyx abiens in fructum. Tournef.

c. Receptaculum cingens germon, s. fructum : ut in Saxifragis variis.

d. Recepiaculum anbiens germen dilatatum sursum per Perianthium, ut in Icosandris, Ribe, Iritella, \&c.

Perianthium, et Corolla juxta se invicem approximata oriuntur, ita ut, sì Corolla germini insideat, vel subjiciatur, idem de Perianthio fiat. Exceptio rara \$. I 00. 
Hepaticce. Perianthium ipsâ basi a Corollâ remotum est; ergo vix perianthium erit, sed involucrum; quod confirmat affinis Pulsatilla.

Germen ante foecundationem parvum est, dein excrescit in magnum.

Musa singularis, cujus germina ante florescentiam maxima, non foecundata sæpius maturescunt sterilia, quamvis mole vix augeantur.

98. DIFFERENS Structura (92) Fructificationis ab iis partibus, quæ in diversis sæpe differunt plantis, desumitur.

Hæc Fundamentum Generum, eorumque Characterum erit. Quo Classis magis Naturalis, eo minùs manifesta est hæe structura.

Singularis omnis structura est differens, sed non vice versa.

99. CALYX differt (98) quoad a. Numerum, Compositionem, Partes, Lacinias: $\beta$. Figuram, Æqualitatem, Marginem, Apicem $: \gamma$. Proportionem. $\delta:$ Locum, Durationem.

Numerus: Nullus: Tulipa, Fritillaria, Liliacei plurimi.

Unicus : Primula, Plerique flores.

Geminus: Malva, Hibiscus; Bixa.

Compositio: Imbricatus, ex squamis variis superimpositis: Hieracium, Sonchus, Camellia.

Squarrosus ex squamis undique divaricatis patentissimis: Carduus, Onopordum, Conyza.

Auctus (Calyculatus Vaill.), ubi series foliorum brevior, diversaque basim calycis exteriùs cingit: Coreopsis, Bidens, Crepis, Dianthus.

Multiflorus, communis multis flosculis: Scabiosa, Syngenesia.

Partes: Monophyllus: Datura, Primula.

Diphyllus: Papaver, Fumaria (etiam bulbosa).

Triphyllus: Tradescantia.

Tetraphyllus: Sagina, Epimedium, Tetradynamia. Pentaphyllus: Cistus, Adonis, Cerbera.

Hexaphyllus: Berberis.

Decaphyllus: Hibiscus. 


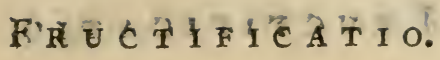

Lacinice: præsertim in Monophyllis numeratæ.

Integer: Genipa.

Bifidus: Utricularia.

Trifidus: Alisma, Cliffortia.

Quadrifidus: Rbinanthus.

Quinquefidus: Nicotiana.

Sexfidus: Pavia.

Octofidus: Tormentilla.

Decemfidus: Potentilla, Fragaria.

Duodecemfidus: Lytbrum.

Equal. AEqualis: Lychnis.

Inæqualis : Helianthemum, Tournef:

alternis brevioribus : Tormentilla, Potentilla.

Figura: Globosus: Cucubalus.

Clavatus: Sitene.

Reflexus: Asclepias.

Erectus: Primula, Nicotiana.

Margo: Integerrimus: Plerisque.

Serratus : Hyperici. species.

Ciliatus: Centaurea sp.

Apex : Acutus: Primila, Androsace.

Acuminatus: Hyoscyamus.

Obtusus:- Nympheea, Garcinia.

Unico denticulo truncato: Verbena.

Proportio: Corollâ longior: Agrostema, Sagina, Antirrbini sp.

Corollæx æqualis : Cerastii sp.

Corollâ brevior : Silene.

Locus: Floris: Linnee, Morina.

Fructûs: Linncea, Morina.

Fructiflcationis: Preonia.

Duratio: Caducus, ad primam explicationem Floris: $P_{x-}$ paver, Epiinedium.

Deciduus cum Corollâ: Tetradjnamia, Berberis.

Persistens ad maturitatem. Fructûs: Didynamia.

INVOLUCRUM: Monophyllum:: Bupleurum.

Diphyllum: Euphorbia..

Triphyllum:- Butomus, Alisma..

Tetraphyllum : Cornus.

Pentaphyllum: Daucus.

Hexaphyllum: Hremanthus.. 
SPATHA: Monophylla: Narcissus.

Diphylla: Stratiotes.

Imbricata : Musa.

₹00. COROLLA differens (98) est, quoad: a. Petala, Lacinias, Nectaria (I IO) : F3. Figuram, Equalitatem, Marginem: $\gamma$. Proportionem: $\delta$. Locum, Durationem.

Pualla: Guorum numerus ex Systemate Rivini constat.S.6I.

Monopetala: Convolvulus, Primula.

Dipetala: Circoa, Commelina.

Tripetala : Alisma, Sagittaria.

Tetrapetala: Tetradynamia.

Pentapetala : Umbellata.

Hexapetala: Tulipa, Lilium, Podophyllum.

Enneapetala: Thea, Magnolia, Liriodendron.

Dolypetala : Nymphraa.

Lacinia: Polypetalis rariores; Monopetalis frequentes:

priorum

Dux: Alsine, Circaa.

Tres: Holosteum, Hypecoum.

Quatuor: Lychnis.

Quinque: Reseda.

Nectaria: vide de his. S. I Io.

Figura: Undulata: Gloriosa.

Plicata: Convolvulus.

Revoluta: Asparagus, Medeola.

Torta: Nerium, Asclepias, Vinca, et ord. nat. 29. Equalit. Equalis: Primula. Inæqualis, Butomus.

Regularis: Aquilegia.Irregul. Aconitum, Lamium. MIargo: Crenata: Linum.

Serrata:Tilia, Alisma.

Ciliata: Ruta, Menyanthes, Tropcoolum.

Denticulis interjectis: Samohus, Sideroxylum, Asperifolie Raj.

Hirsuta superficie:Menyanthes, Hyperici Lasiantbus.

Proport. Longissima : Catesbrea, Siphonanthus, Brunsfelsia, Craniolaria.

Brevissima: Sagina, Centunculus, Ribes.

Locus: Corollze basis approximaturPerianthio, modò adsit. Rara Exempla, quòd Corolla a Calyce remota, interjecto Germine, ut Adoxa, Sanguisorba, Mirabilis. 
Duratio: Persistens (ad maturitatem Fructûs): Nymphrea.

Caduca(ad explicationemFloris): Actcea,Thalictrum. Decidua (cum Floris casu): Plerceque.

Marcescens (contabescit, nec decidit): Campanula, Orchis , Cucumis, Cucurbita, Bryonia, et affines, ord. nat. 45 .

ror. STAMINUM Filamenta differunt (98) quoad $\alpha_{0}$ Numerum: $\beta$. Figuram : $\gamma$. Proportionem: $\delta$. Situm. Antberce autem quoad a. Numerum: Loculamenta, Defertum : $\beta$. Figuram, Dehiscentiam: $\gamma$. Connexionem: $\delta$. Situm.

Filamenta. Numerus differt, ut in Systemate sexuali.

Lacinic: 2. Salvia, 3. Fumaria, 9. Diadelpbia.

Figura: Capillaria: Plantago.

Plana: Ornitbogalum.

Cuneiformia : Thalictrum.

Spiralia: Hirtella.

Subulata: Tulipa.

Emarginata: Porrum.

Reflexa: Gloriosa.

Hirsuta: Tradescantia, Anthericum.

Proportio: Inæqualia:Daphne, Lychnis, Saxifraga. Irregularia: Lonicera, Didynamia.

Longissima : Trichostema, Plantago, Hirtella.

Brevissima: Triglochin.

Situs: Calyci opposita: Urtica.

Calyci alterna: Elceagnus.

Corollæ inserta : in Monopetalis, vix in Polypetalis.

Calyci inserta: interdum in Apetalis, uti in Elceagno, et semper in Icosandris, et Oenothera: aftinibusque ord. nat. 40.

Receptaculo communiter inseruntur, uti Calyx , et Corolla.

Anthera numero Unica in singulo filamento: Plerique. in filamentis tribus: Cucurbita. in filamentis quinque: Syngenesia.

Dur in singulo filamento: Mercurialis. 
Tres in singulo filamento: Fumaria, Quinque in tribus filamentis: Bryonia, in singulo filamento: Theobromate

Locuio Unico: Mercurialis.

Gemino: Helleborus

Trino: Orchis.

Quaterno: Fritillaria.

Defectu Unius: Chelone, Martynia.

Duorum: Pinguicula, Verbena.

Trium: Gratiola; Bignonice, Geranid:

Quatuor: Curcuma.

Quinque: Pentapetes, Gerania.

Figura Oblonga: Lilium.

Globosa: Mercurialis.

Sagittata: Crocus.

Angulata: Tulipa.

Cornuta: Hamametis, Erica, Vaccinia, Pyrola.

Debiscentia Latere; Plerique, Leucojum. Apice: Galantbus, Kiggelaria.

a Basi ad Apicem discedens : Epimedium, Leontice.

Connexione Apice: Colchicum.

Basi: Plerique.

Latere: Canna.

Nectario: Costus.

Situs in Filamentis apice: Plerique.

latere: Paris, Asarum.

Pistillo: Aristnlochia.

Receptaculo: Arum

Polien Figura: globus echinatus: Helianthus. perforatus: Geranium.

didymus: Symphytum.

rotatus dentatus: Malva.

angulatus: Viola.

reniformis: Narcissus.

Philyra convoluta: Borrago. 
ro2. PISTILLA differunt (98) quoad $\alpha$. Numerum, Lacinias: $\beta$. Figuram: $\gamma$. Longitudinem, Crassitiem $: \delta$. Situm, trium scilicet partium.

Partes tres Pistilli: Germen, Stylus, Stigma.

Germen observat Leges Pericarpii hinc videndum \$. I03. Stylus semper a Calyce, et Corollâ distinctus.

Numerus videndus in Systemate Sexuali, ubi Pistillorum

Numerum a stylis (si adsint, aliàs a stigmatibus) desumsi.

$\begin{aligned} & \text { Lacinia: } \text { Bifidus: Persicaria, Cornutia. Dichotomus: Cordia. } \\ & \text { Trifidus: Clethra, Frankenia. }\end{aligned}$

Quadrifidus: Rhamni.

Quinquefidus: Geranium.

Figura : Cylindricus: Monotropa. Angulatus: Canna. Subulatus: Geraniun. Capillaris: Ceratocarpus.

Supernè crassior: Leucojum.

Longitudo : Longissimus : Tamarindus, Cassia, Campanula, Scorzonera, Zea.

Brevissimus: Papaver.

Staminum longitudine: Nicotiana, Plerique.

Crassities: Staminibus crassior: Leucojum.

tenuior: Ceratocarpus.

æqualis: Lamium.

Situs: in apice Germinis: Plerique; hinc reticendus. supra infraque Germen: Capparis, Euphorbia.

Nisi inferiorem partem pro Receptaculo elongato sumas.

ad latus Germinis : Icosandria polygynia, ejusque affines: ord. nat. 35 . Hir-

Duratio: Persistens: Tetradynamia. tella, Suriana.

Stigma numero unicum: Plerisque.

Duo: Syringa.

Tria: Campanula.

Quatuor: Epilobium, Parnassia.

Quinque: Pyrola.

Lacinic: convoluta: Crocus.

Capillares: Rumex.

revoluta: Syngenesia, Dianthus, Campanula.

Flexa sinistrorsum: Silene.

Sexpartitum: Asarum.

E 2

Mul- 


\section{Multifidum: Turnera.}

Figura: Capitatum: Tribulus, Hugonia, Vinca, Ipo-

$$
\text { mrea, Clusia. }
$$

Globosum: Primula, Hottonia, Linnaa, Li-

$$
\text { mosellu. }
$$

Ovatum: Genipa. il

Obtusum: Andromeda.

Truncatum: Miaranta.

Obliquè depressum: Actca, Daphne.

Emarginatum: Melia.

Orbiculatum: Lythrum.

Peltatum: Sarracena, Nymphea, Clusia, Papaver.

Coroniforme: Pyrola.

Cruciforme: Pencea.

Uncinatum: Viola, Lantana.

Canaliculatum: Colcbicum.

Concavum: Viola.

Angulatum: Muntingia. Striatum: Papaver.]

Plumosum: Rbeum, Gramina, Triglockin,

Tamarix.

Pubescens: Cucubalus, Lathyrus.

Longitudo: Filiformis: Zea.

Longitudine styli: Genipa.

Crassities: Foliacea: Iris.

Duratio: Persistens: Sarracena, Hydrangex, Nymphoea, Papaver.

Marcescens: communiter.

Stamina a stylo distinctissima sunt in omni flore.

Canna, Alpinia, et quibusdam ord. nat. 3. exceptis, ubi cohærent stylo.

Gynandre etiam cohærent, sed aliter.

ro3. PERICARPIUM differt (98) quoad a Numerum, Loculamenta, Valvulas, Dissepimenta: $\beta$. Species, Figuram, Dehiscentiam: $\gamma$. Inclusionem: d. Situm.

Numerus externè dividit fructum in plures partes, non verò internè.

Nullum Pericarpium: Gymnospermum. Herm. Semina nuda. Riv: Thymus.

Unicapsularis: Lycbnis.

Bicapsularis: Pceonia, Asclepias. 
Tricapsularis: Veratrum, Delplinium.

Quadricapsularis: Rhodiola.

Quinquecapsularis: Aquilegia.

Multicapsularis: Calt ba, Trollius, Hellebori.

Loculamenta internè dividunt fructum, non externè.

Unilocularis: Trientalis, Primula.

Bilocularis: Hyoscyamus, Sinapis, Nicotiana.

Trilocularis: Lilium.

Quadrilocularis : Evonymus.

Quinquelocularis: Pyrola.

Sexlocularis: Asarum, Aristolochia.

Octolocularis: Radiola Lini.

Decemlocularis: Linum.

Multilocularis: Nymphrea.

Valvulce externos parietes respiciunt, uti Dissefimentun loculamenta.

Bivalvis: Chelidonium, Brassica.

Trivalvis: Viola, Polemonium, Helianthemum.

Quadrivalvis: Ludzuigia, Oenothera.

Quinquevalvis: Hottonia.

Dissepimentum Parallelum: Lunaria, Draba.

Species, vide \$. 86. n. 5.

$$
\text { contrarium: Eiscutella, Thlaspi. }
$$

Figura, Turbinata:

Inflata: Cardiospermum, Staphyleea.

Membranacea: Ulmus.

Triquetra, Tetragona, Pentagona : Averrboa, Zygopbyllum.

Articulata: Ornithopus, Hedysarum, Raphanus.

Debiscentia, quum fructus maturus semina dispergat.

Apice Quadridentato: Dianthus.

Quinquedentato: Alsine.

Decemdentato: Cerastium.

Basi Trifariam: Triglocbin, Campanula.

Quinquefariam: Ledum.

Angulis longitudinaliter: Oxalis, Orchis.

Poro: Campanula.

Horizontaliter: Anagallis, Plantago, Amaranthus, Portulaca, Hyoscyamus.

Articulatus omnis fructus dehiscit secundùm articulos monospermos: Hypecoum, Hedysarum, Ornithopus, Scorpiurus, Raphanus. 
Inclusio, Elasticè: Oxalis, Elaterium, Momordica, Impatiens, Cardamine, Pbyllantbus, Eupborbia, Justicix, Ruellia, DiEzannus, Hura, Ricinus, Tragia, Jatropha, Crolon, Clusia, Acalypha.

Situs ad receptaculum floris, idque vel

Infra: Vaccinium, Epilobium.

Supra: Arbutus, Tulipa.

Supra, infraque: Saxifraga, Lobelia.

104. SEMINA differre observantur quoad «. Numerum, Loculamenta; $\beta$. Figuram, Substantiam, Coronulam, Arillum; $\gamma$. Magnitudinem; $\delta$. Córculum; $\varepsilon$. Receptaculum.

Nuinerus ex Systemate Rivini desumendus.

Monosperma: Polygonum, Collinsonia.

Disperma : Umbellate, Stellate.

Trisperma : Euphorbia.

Tetrasperma: Asperifolice, Vericillate.

Loculamentum plerisque unicum.

Biloculare: Cornus, Xanthium, Locusta, Valeriana, Cordia.

Figura: Cinctum: Arenaria, Bryoniá.

Cordiforme, Reniforme, Ovatum.

Echinatum: Myosotis Lappula.

Substantia Ossea : Nuces, Corylus, Lithospermum.

Callosa: Citrus.

Coronula, Calysulus ex perianthio floris: Scabiosa, Knautia, Ageratum, Arctotis.

Pappus Capillaris ( simplex, filiformis) Hieracium, Soncbus.

Plumosus (villosus: compositus) Crepis, Scorzonera, Tragopogon.

Paleaceus: Bidens, Silphium, Tagetes, Coreopsis.

Nullus (Nudum semen): Tanacetum.

Arillus quibusdam Calyptra dictus: Coffea, Jasminum, Cyno. glossum, Cucumis, Dictamnus, Diosma, Celastrus, Evonymus.

Magnitudo minima: Campanula, Lobelia, Trachelium, Ammannia;-maxima: Coccus.

Situs: Nidulantia; per pulpam sparsa : Nymphea.

Suturæ adnexa: Siliquose. 


\section{FRUCIIFICA TIO.}

Columellæ adfixa: Malva.

Receptaculis insidentia: Nicotiana, Datura. Receptaculum compositorum præcipuè investigandun.

Figurâ Planum: Achillea.

Convexum : Matricaria.

Conicum: Anthemis, Melampodium.

Superficie nudâ: Matricaria.

Punctatâ : Tragopogon.

Villosâ: Andryala.

Setosâ: Centaurea.

Paleaceâ: Hypocharis, Anthemis.

Simplicium Receptacula fructûs singularia in Magnolia, Uvaria, Michelia.

Hilum evidentissimum in Cardiospermo, Staphylea.

Hilo proximum est Corculum.

Sedes Corculi est vel in apice, vel basi Seminis.Cresalpin. Plantula gignitur, vel qua parte Pedunculus adheret, vel prorsus contra. Quæ gignitur qua parte Pedunculus adhæret, exporrigit ad Pedunculum radices, folia autem ad apicem. Quæ verò gignitur in apice, folia extendit pedunculum versùs, radices autem ad apicem. Foseph.ab Aromatariis, de Seminibus 4.5.6.

105. SINGULARIS (92) Fructificatio ab eấ structurå, quæ in paucissimis Generibus obsévvatur, desumitur.

Opponitur Structuræe Naturali. \$.93.

Exempla: Aro Stamina intra Pistilla.

Adoxee Germen intra Calycem, et Corollam.

Salvice Filamenta articulata.

Eriocaulo Stamina Germini insidentia, Corolla, et

Calyx Germini subjecta.

Magnolice Receptaculum fructûs capitatum, seminibus baccatis in filo pendulis e capsula.

ro6. CALYX Corolla minùs coloratus esse solet.

Perianthium hîc primariò intelligitur ; secundario Involu-

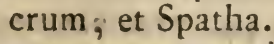

Ratio patet ex materiâ Calycis, ex cortice plantỉ natâ. Exempla rariora contrarium indicantia:

Bartsia ainericana: Perianthium sanguineum.

E 4 
Cornus herbacea:Involucrum niveum; Petala nigra. Americana: Involucrum rubrum cordatum.

Astrantia: Involucrum coloratum.

Palme: Spathæ sanguineæ.

Ubi Corolla deest, Perianthium communiter magis coloratum esse solet, præsertim tempore florescentiæ, ut in Ornithogalo, Persicariâ, Polygono.

Caly x, aut Corolla minùs colorata ubi observantur, ibi folia sæpe colorem induunt, ut in Amarantbo tricolore.

107. RECEPTACULUM Floris (25) Periantbium internè cingit in Icosandris, aliisque; adnascitur undique in Cucurbitaceis $(77: 45)$.

Receptaculi consideratio magni momenti res in methodo naturali est.

Pleræque Plantæ Receptaculum floris, s. Stamina, et Petala fundo floris inserunt.

Icosandre(Ord. nat. 45.46.47.48.) Calycem monophyllum habent, quem internè undique cingit linea, cui Stamina, et Petala adnectuntur.

Calyces ejusmodi floriferos etiam in aliis observamus; Lytbrun, Epilobium, Oenothera, Ammannia, Isnarda, Peplis, Elceagnus.

Eucurbitacece (Ord. Nat. 45). Receptaculum in his interne obvestit: undique Perianthium, cui Corolla quasi adglutinatur.

Idem in Cactis obtinet.

Receptaculum elevans Pericarpia: Passifora, Capparis, Breynia, Arum, Calla, Dracontium, Pothos, Zostera, Nepenthes, Cluna; Helicteres, Sisyrinchium.

108. FILAMENTA Staminum a Corollâ Palypetalâ distincta, Corollæ vero Monopetalæ inserta sunt: exceptis antheris bicornibus.

Vaillantius hoc ob ervavit in Monopetalis.

Pontedera ex dissectione 2000.specierum didicit Flores Manopeta los gerere stamina corolle inserta, at Poly petalos receptaculo floris.

Tournefortius MIalvaceos (Ord. Nat. 44.) verè pentapetalos, uti ex basi corolla constat, inter monopetalos refert. Rivinus tot nunierat Petala, in quot sponte sua resolvitur flos deciduüs. 


\section{FrRU CTIFICATIO.}

Monopetali Flores Stamina Petalo inserunt:

Sic Trientalis monopetalus est.

Oxalis, vix basi petalorum cohærens, monopetala evadit.

Polypetali Stamina a Petalis distincta gerunt.

Exceptio tamen admittitur rariùs:

Statice pentapetala filamenta unguibus petalorum inserta habet.

Melantbium hexapetalum filamenta gerit petalis inserta.

Lychnides (Ord. nat. 42.) alterna stamina unguibus petalorum sæpius adnectunt.

Monopetalos flores dari staminibus a corollâ separatis in Bicornibus (Ord. nat. 24.) docent: Erica, Andromeda, Arbutus, Ledum, Azalea, \&c. uti et Cissus, Aloë.

rog. ANTHER死 apici Filamentorum communiter insident.

Exceptio rarior, ubi Antheræ lateri filamenti adglutinantur: Paris, Asarum.

Antheræ stigmati adhærent absque filamentis, in Aristolocbia.

110. NECTARIUM, si a Petalis distinctum, communiter ludit.

Humor melleus secernitur in plerisque floribus.

Monopetalorum tubus plerumque mel continet.

Pontedera statuit hunc liquorem esse colliquamentum, s. Amnii liquorem, qui intrabat semina focunda; sed datur etiam mel

in Roribus Masculis: Urtica, Salix.

in Femineis tantùm: Phyllanthus, Tamus.

in utroque Sexu: Rusco, Clutia, Kiggelaria.

Vaillantius Nectarium essentiale Corollæ esse statuit; sic Nigellæx, Aquilegixe Nectaria dixit petala esse. Petala verò pro calyce habuit.

Distincta esse Nectaria a Corollà, constat exemplis: Aconitumi, Aquilegia, Hellevorus, I sopyrum; Nigella.Garidella; Epimedium, Parnassia, Theobroma; Cherleria, Sauvagesia. Calcarata Monopetala: Antirrbinum, Valeriana, Pinguicula, Utricularia.

Polypetala : Orcbis, Dilpbinitum, Viola, Impations, Fumaria. 
Corollæ petalis internè : Fritillaria, Lilium, Swertia, Iris, Hermannia, Uvuluria, Hydrophyllum, Myosurus, Ranunculus, Bromelia, Ery. thronium, Berberis, Vallisneria.

Coronantia Corollam: Passiflora, Narcissus, Pancratium, Olax, Lychnis, Silene, Coronaria, Stapelia, Asclepias, Cynanchum, Nepenthes, Cherleria, Clusia, Hamamelis, Diosma.

Singulari modo constructa: Reseda, Cardiospermum, Amsmum, Costus, Curcuma, Grewia, Urtica, Andrachne, Epidendrum, Helicteres, Salix.

Calycina: Tropeolum, Monotropa, Biscuiella, Malpighia. Staminea Antheris: Adenantherá.

Filamentis: Laurus, Dictamnus, Zygophyllum, Commelina, Mirabilis, Plumbago, Campanula, Roëlla.

Pistillacea Germinis: Hyacinthus, Iris, Butomus, Cheirantbus, Hesperis, \&c.

Receptaculacea : Latbrea, Helxine, Collinsonia, Sedum, Cotyledon, Sempervivum, Ëc. Mercurialis, Kiggelaria, Clutia, Phyllanthus, Melianthus, Diosma.

I I. PISTILLUM intra Antheras communiter collocatur.

Arum singulare est, ubi, elongato receptaculo in clavam, Pistilla basim occupant, Stamina verò superiorem partem; adeoque Pistilla extra stamina, et circum stamina collocantur.

Calla cethiopica eodem modo se habet.

Rumex singularis est insertione staminum.

I12. STYLUS apici Germinis communiter insidet; exceptis paucis.

fungius 39. Stylus semper apici fructûs, seminisque cohæret. Dillenius, respons. 6. Nullum dari stylum, qui non e medio floris, ex medio embryone, qui medium floris occupat, oriatur, cuique Tyroni notissimum.

Excipiuntur varii flores:

I. Icosandre polygynce (Ord. Nat. 35.) Rosa, Rubus, Fragaria, Potentilla, Tormentilla, Dryas, Geum, Comarum, Sibbaldia, Agrimonia, Alchemilla, Aphanes, 
2. Suriana, Hirtella.

3. Passerina, Gnidia, Strutbia, Stellera.

1. 3. PERICARPIUM naturaliter clauditur, nec repletur minoribus Pericarpiis, sed sæpius succulentum transit in BaccAm.

Clauditur in plerisque exactè.

Reseda, et Datisca tamen semper hiant.

Parnassia sub fiorescentia hiat, deinde clauditur.

Fotum dari Pericarpium naturaliter minoribus pericarpiis, mihi non constat; ubi autem Pericarpia quasi latent intra aliud, tum exterius Receptaculum commune est : ut in Magnolia, Uvaria, Michelia.

Bacca est succulentus fructus propriè ex Pericarpio facta, impropriè ex parte qualibet. Finis Baccæ, ut semina ab animalibus serantur: e. gr. Viscum.

Singulares, et impropriæ Baccæ sunt sequentes; ubi fit Calyx: Blitum, Morus, Easella, Ephedra, Coix, Rosa, Coriaria. Receptaculum: Taxus, Rhizophora, Anacardium, Ochna,

Laurus, Ficus, Dorstenia, Fragaria.

Semen : Rubus, Magnolia, Uvaria, Michelia, Prasium, Uvularia, Panax, Adonis, Crambe, Osteospermum. Arillus: Evonymus (Celastrus hoc docuit).

Nectarium : Mirabilis.

Corolla: Poterium, Adoxa, Coriaria.

Capsula:Evonymus, Androscemum T.Cucubalus, Epidendrum. Sicca Bacca : Linncea, Galium, E'c. Tetragonia, Myrica, Trientalis, Tropeolum, Xanthium, Juglans, Ptelea, Ulmus, Comarum, Amygdalus, Mirabilis.

Capsula externè : Dillenia, Clusia, Nymphara, Capparis, Breynia, Morisona, Stratistes, Cyclamen, Strychnos.

Cava: Stapbylaca, Cardiospermum, Capsicum.

Conceptaculum: Actoa.

Legumen: Hymencea, Cassia, Inga Pl. Ceratonia.

Strobilus: Annona, Funiperus.

Bacca naturaliter non dehiscit; quia mollis, et alius finis. Baccæ aggregatæ manifestissimæ in Adonide capensi.

I 4. COMPLETI Flores sunt Simplices, aut Aggregati.

Divisio Florun Vaillantii lixe erat:

Completus Flos gaudet Perianthio, et Corollâ. 
Incompletus caret Perianthio, aut Corollà. Apetalus Flos caret Corollâ, non Perianthio.

Nudus Flos caret Calyce; non Corollâ.

Dicitur magis aptè nudus, ubi et Corollà, et Calyce caret, quod tamen rarissimum est.

Primaria, et maximè naturalis plantarum divisio ex Cotyledonibus desumta fuit, in Monocotyledones, et Polycotyledones; ubi divisio inter simplices, et propriè compositos flores sit, præsupponitur plantam esse polycotyledonem.

Ir5. SIMPLEX Flos, cùm pluribus floribus nulla pars fructificationis communis est.

Simplex flos intra Perianthium, aut Corollam unicum Thalamum constituit.

Fructus compositus, seu multicapsularis nequit florem compositum constituere.

II6. AGGREGATUS Flos, cùm flosculis pluribus aliqua pars fructificationis communis est; diciturque Aggregatus propriè, vel Compositus, vel Umbellatus, vel Cymosus.

Aggregatus fit flos, cùm plures flosculi mediante aliquâ parte fructificationis omnibus communi ita uniuntur, ut alter flosculus demtus destruat formam totius, cujus pars est. Commune in his est Receptaculum, aut Calyx.

Flosculus dicitur flos partialis floris aggregati.

Modi florum aggregatorum septem primarii sunt.

r. Umbellatus flos habet Receptaculum divisum in pedunculos, omnibus eodem centro exeuntibus.

2. Cymosus flos habet Receptaculum divisum in pedunculos ex eodem centro universali enatos, pedicellis verò vagè prodeuntibus.

3. Compositus flos habet Receptaculum dilatatum integrum, fosculis sessilibus.

4. Aggregatus flos (propriè dictus) habet Receptaculum dilatatum, flosculis insidentibus pedunculis : e. gr. Scabiosa, Enautia, Dipsacus, Cephalanthus, Globularia,

Leucadendron, Protea, Brunia, Barreria, Statice T.

5. Amentaceus aggregatus fos habet Receptaculum filiforme distinctum squamis amentaceis: 


\section{FRUCTIFICA II $O$}

Xantbium, Ambrosia, Partbenium, Itis.

Alnus, Betula.

Salix, Populus.

Corylus, Carpinus.

Juglans, Fagus, Quercus, Liquidambar.

Cynomorium.

Ficus, Dorstenia, Parietaria, Urtica.

Pinus, Abies, Cupressus, Thuja.

Juniperus, Taxus, Ephedra.

6. Glumosus aggregatus flos habet Receptaculum filiforme, cujus basis instruiturr gluná communi.

Bromus, Festuca, Avena, Arundo, Briza, Poa, Aira, Unicla, Cynosurus, Melica, Elymus, Lolium, Triticum, Secale, Hordeum, Scirpus, Cyperus, Carex.

7. Spaticeus aggregatus flos est, ubi Receptaculum intra spatham, pluribus flosculis communem.

Spadix subdivisus est in Palmis.

Simplex tectus undique flosculis : Calla, Dracontium, Pothos.

infernè : Arum.

altero latere : Zostera.

i17. COMPOSITÚS Flos est Aggregatus (ir 6 ) continens flosculos plures sessiles, Receptaculo communi integro, et Perianthio contentos, sed Antheris in cylindrum connatis instructos.

Proprietates foris compositi sunt:

a. Receptaculum commune ampliatum indivisum.

b. Perianthium commune flosculos onnes cingens.

c. Antherx 5 in cylindrum connatz.

d. Flosculi sessiles monopetali.

$e$. Gefmen monos permum proprium sub singulo fośculo.

Essentiale compositis est, habere antheras connatas in cylindrum, et semen unicum sub singulo flosculo.

Observa; 'dari cómpositos fores, quorum calyces unico flosculo instrúuntur, e. gr. Ećbinop's , Stäbe, Corymbium, Artemisia ranica.

Triplices numerantur vilgò fores compositi.

a. Liguzati (Semiflos zulosi, Tourn.) cùm corollulæ floseulorum om nes planx, versùs exterius latus expansx sunt. b. Turuzosr (Flosculosi Tournef.) cum corollule flosculorum omes tubulosæ subæqualés sunt. 
6. RADIATE, cùm Corollulæ disci tubulosæ, in ambitu verò flosculi difformes sunt:

vel in ambitu flosculis ligulatis (Radiati Tournef.)

vel in ambitu flosculis tubulosis: Centaurea.

vel in ambitu flosculis subnudis Artemisia,

Ginaphalium.

Constat compositus Flos sæpius flosculis pluribus, rariùs numero determinatis.

Ligulatus flosculis 5. Prenanthes.'

Tubulosus flosculis 20. Eupatorium Scrophularice folio.

15. Eupatorium perfoliatum.

5. Eupatorium digitaium.

Eupatorium Zeylanicum.

Eupatorium secund. Hort. Ups. Eupatorium quart. Hort. Ups. 4. Eupatorium volubile.

Radiatus flosculis radii multis: Pler aque.

20. Arctotis.

12. Rudbeckia.

I0. Tetragonotheca, Osteospermum.

8. Coreopsis, Othonna.

5. Achillea, Eriocephalus, Micropus, Seriphium, Sigesbeckia, Acmella, Melampodium, Chrysogonum, Tagetes.

3. Sigesbeckia.

1. Milleria, flosculis disci 3.

I 8. UMBELLATUS Flos ( I 16) est Aggregatus ex flosculis pluribus insidentibus receptaculo in pedunculos fastigiatos, omnes ex eodem puncto productos.

CYMA ( I I6) verò flos est Aggregatus ex flosculis pluribus insidentibus receptaculo in pedunculos fastigiatos, primores ex eodem puncto productos, posteriores autem sparsos.

Umbella est, ubi Pedunculi omnes ex eodem centro ambitu æquali exeunt.

- Simplex umbella, ubi receptaculum sic semel dividitur in pedunculos: Cornus, Spircere species. 
Conposita umbella, ubi omnes pedunculi communes subdividuntur in umbellulas.

UMBELLULA itaque partialis umbella est.

Proprietates umbel latorum florum propriè sic dictorum sunt: A. Receptaculum commune divisum in pedunculos e centro, seu puncto eodem ortos ambitu xquales, sive inde umbella plana, seu convexa, seu concava evadat.

b. Germen sub corollulâ.

c. Stamina 5 distincta, decidua.

d. Pistillum bifidum.

e. Semina duo connexa summitate.

Constat involucro, umbellâ, Radio diversis. Involucrum universale 4 phyllum: Hydrocotyle, Sison, Cuminum. sphyllum: Bupleurum, Scandix, Bubon. 7 phyllum: Ligusticum. Iophyllum: Artedia.

partiale dimidiatum : Etbus.t, Coriandrum, Sänicula. caducum : Ferula, Heracleum.

Umbella disco másculo: Astrantia, Caucalis, Artedia, Onnanthe, Scandix.

Radiata, petalis marginalibus majoribus : Tordylium,

Caucalis, Coriandrum, Ammi, Heraclei species.

Crya, uti Umbella, omnes pedunculos primarios, ex eodem centro educit, partiales verò vagos spargit: Opulus, Cornus virga sanguinea dicta, Ophiorrhiza.

Receptaculum sic productum esse, docet Involucrum; demonstrat Cornus umbellata, cujus species Virga Sanguinea; qux uti Tinus, aut Viburnum pedunculis ramificatur.

i 19 . LUXURIANS Flos Tegmenta fructificationis ita multiplicat, ut essentiales ejusdem partes destruantur; estque vel Multiplicatus, vel Plenus, vel Prolifer. Mutilus autem dicitur is Flos, qui. Corollan excludit.

Tegmentum floris est Perianthium, et Corolla.

Fit luxurians, dum alix partes numero augentur, ut aliæ. excludantur.

Oritur luxurians flos plerumque ab alimento luxuriante.

Mutilus flos nobis est, qui corollam non promit, quamquam eamdem promere deberet; hoc autem plerumque fit a defectu sufficientis caloris. 
Ipomac. F1. Zeyl. 79.

Campanula pentagonia. Hort. Ups. n. 3.

Ruellia. Hort? ups. $\mathbf{1 7 9 .}$

Vinle varice. (Diss. Sem. Muscor.)

Tussilago Anandria.

Cucubalus F1. suec. 363. lapp. I 8 I. $_{0}$

I20. MULTIPLICATUS ( I I9) Flos de Corollâ multiplicatâ, salvis quibusdam Staminibus, communiter prædicatur; estque Duplicatus, vel Triplicatus. Periantbium, et Involucrum rarò ; Stani $=$ na vix unquam multiplicatum constituunt florem.

Distinguo Multiplicatum Aorem a pleno, quòd in Pleno Corolla ita multiplicata est, ut staminibus vix ullus locus. Multiplicatus verò prædicatur de serie duplici, triplici, aut quadruplici corollæ auctiore.

Duplicatus flos, adeoque primus, et minimus gradus plenitudinis est.

Triplicatus corollâ triplo auctior evadit.

Campanula fol. urtice, flore duplici, et triplici Tournef. Stramonium flore altero alteri innato Vaill.

Stramonium flore violaceo duplici, triplicive Tournef.

Monopetali sæpius multiplicantur; rariùs pleni evadunt. Polypetali non rarò multiplicantur; e. gr. Hepatica, Anemone. Perianthium rarò multiplicatum florem constituit.

Dianthus Caryophyllus spicam frumenti referens. E. N. C. cent 3. p. 368 . t. 9. In hoc squamæ calycinæ augentur in infinitum, ut constituant integram spicam modo singulari. Vid. Hort. Cliff. 164.

Gramina alpina quasi plena evadunt, dum glumx in folia excrescunt : Festuca spiculis viviparis. Fl. suec. 94. Salix rosea, ubi, destructis staminibus, aut pistillis ab insectis, amenti squamæ in folia enascuntur.

Plantago rosea, cùm bracteæ spicæ excruscunt in folia.

Caveas, ne Perianthium coloratum pro multiplicato sumas, licèt sit gradus monstrositatis; e. gr.

Primula prolifera odorata. Tournef.

Primula prolifera, flore majore. Tournef.

Primula prolifera, flore purpuroo. Tournef. 


\section{- FrútITICATio. - 8I}

r2I. PLENÚS (I I9) Flos, cùm Corolla adeo multiplicatur, ut Stamina omnia excludantur.1

Fit, dum Stamina excrescunt in Petala, hre replent flores, et sæpius suffocant pistillum, præsertim, dum omnia stamina exclusa sunt.

Polypetali sæpius fiunt pleni:

Malus, Pyrus, Persica, Cerasus, Anygdabus, Mryriuss, Rosa, Fragaria.

Ranunculus, Caltha, Hepatica, Arsmon, Aquilegia, Nigella. Papaver, Peonia; Dianthus, Silene, Lycbnis, Corondviv. Lilium, Fritillaria, Tulipa, Narcissus, Colchicum, Crocus. Cheiranthus, Hesperis; Malva, Alcea, Hibiscus.

Monopetali rariùs implentur:

Primula, Hyacinthss, Datura, Polyantises.

Steriles esse plenos, et ratio $\$$. I 50.

Genera non constituere plenos \$. i 84.185 .

Anthophilorum, et Hortulanorum delicize suntr flores Pleni.

122. Multi plantarum ordines naturales fores luxuriantes exhibere nequeunt.

Ejusmodi sunt præprimis sequentes:

Apetalce (ord. nat. 48. 53).

Verticillatice (ord. nat. 58.)

Personate Tournef. (ord. nat. 59.) excepto Antirnbins. Asperifolice Raj. (ord. nat. 43).

Stellate Raj. (ord. nat. 44).

Umbellate Raj. (ord. nat. 22.) exceptâ umbellâ proliferà.

Papilionacea (ord. nat. 55.) rarò plenos flores producunt: ut

Ternatea flore pleno caruleo, $\mathrm{T}$.

Coronilla herbacea fl. vario pleno, $\mathrm{T}$.

Anthyllis vulgaris flore pleno, Nobis visa.

Spartium.

123. PROLIFER (I 19) Flos fit, cùm intra florem (sæpius plenum) alii flores enascuntur. Prolifer autem Frondosus dicitur, cùm Proliferi proles foliosus fit. Prolifer flos, ubi ex uno flore alius enascitur. Proliferi flores fiunt ex causâ plenitudinis auctâ. Proliferi (non compositorum florum) fiunt e Pistillo, adeoquêe e centro floris pleni enascuntur Proles. E $\mathrm{Fr}_{\mathrm{O} \text { - }}$ 
Frondosus prolifer rarissimus, est, visus in Rosa, Anemone \& 8 .

Prolifer autem prole florifero frequens est.

Ranunculus radice tuberosâ, flore pleno, et prolifero. C. B. Ranunculus Asphodeli radice, prolifer, miniatus. C. B. Anemone latifolia, pavo dicta, major, prolifera. C. B.

324. PROLIFICATIO (123) florum Simplicium ( I I 5 ): e Pistillo; Aggregatorum (II6) verò e Receptaculo fit.

Prolificatio fit duplici modo, vel

a. Prolificatio e centro, seu ex Pistillo enato in prolem, uno pedunculo peragitur, fitque in floribus non compositis.

Diantbus: Caryophyllus altilis, major, flore pleno prolifero. Fl. March.

Ranunculus radice tuberosa, $f$. pleno et prolifero, Tourn. tuberosus anglicus poly:anthos. Vaill.

Anemone latifolia, pavo dicta, prolifera. Tournef. pavetta latifolia multiplex. Valent.

Geum flore uno alteri innato. Tournef.

flore triplici, secundo primi, tertio secundi calysi: innato. Tournef.

Rosa rubra prolifera.

b. Prolificatio e latere, ex calyce communi proles plurimos pedunculatos emittens, fit in compositis $\mathrm{Ag}$ gregatis propriè dictis.

Bellis hortensis prolifera. C. B.

Calendula prolifera. C. B.

Hieracium falcatum proliferum, C. B. prodr. 64.

Scabiosa, foliis Gingidii prolifera.

Umbellati, dum prolificantur, augent umbellułam, ut ex umbellùtâ simplici altera exeat.

Cornus: Periclymenum humile fiore flori innato. Act. Haffn IV. p. 346 .

Supratecomposita umbella fit similiter ex Composita.

Selinum: Thysselinum palustre: lactescens, non rarò.

125. IMPLETIO florum simplicium vel Petalis, vel. Nectariis peragitur.

Alia est plenitudo florum Simplicium, Compositorum alia. Aqui- 


\section{FRUCTIFICATIO.}

Aquilegia triplici modo impletur:

a. Petalis multiplicatis; Nectariis exclusis. Aquilegia flore roseo. C. B.

b. Nectariis multiplicatis; Petalis exclusis. Aquilegia flore multiplici. C. B.

c. Nectariis multiplicatis, Petalis permanentibus 5, ita ut petala quinque persistant, his interjecta sunt Nectaria ubique tria, quæ se invicem deglutiunt. Nigella flore pleno: cui

Petala 5 inferiora ovata integra: reliqua implentia multifida triloba plana; ergo hæc a nectariis multiplicatis orta.

Narcissus vel ex Petalis, et Nectario multiplicatis impletur, vel Nectario pleno, petalis non multiplicatis.

Delphinium impletur plerumque Petalis planis, Nectario excluso.

Singularis est metamorphosis Saponarice anglicanæ, qux fit ex Pentapetalâ verè Monopetala.

Maximè Singulariș Peloria.

126. Multiplicantur ( 120 ) sæpius Flores in Corolla

Polypetalâ; Duplicantur autem frequentiùs in

Monopetalâ. Flores tamen Monopetalos esse,

simulque Plenos contradictorium non est.

Framer 6. Monopetalos flores et simul plenos esse contradictiones statuit ; sed negant Colchicum, Crocus, Hyacinthus, Polyanthes.

Flores monopetali per lacinias Limbi; Polypetali per

Petala implentur.

Opulusflore globoso. C. B. rarissimum plenitudinis exemplum suppeditat; Opulus vulga ris enim gaudet Cyma, qux constat flosculis hermaphroditis numerosis campanulatis in disco; in ambitu verò, s. radio floribus sterilibus ex corolla planâ rotatâ; in Opulo flore globoso omnes fores disci evadunt similes radii, scilicet corollis rotatis magnis sterilibus, adeoque more compositorum florum impletur Opulus solâ magnitudine sterilitante. Hinc etiam Cymare natura proximè accedit ad Umbellam, quod et Cornus mas umbellata collata cum Corno femina, s. Ossea indicat. r27. COMPOSITI (I I 7 ) ilores implentur ( $12 \mathrm{I}$ ) vel Petalis tubulatis, vel planis.

Compositi Syagenesiæ flores intelliguntur ex dictis.

$$
\mathrm{F}_{2} \text { Con- }
$$


Constant hi vel corollulis fistulosis (Flosculosis T.), vel corollulis ligulatis (Semiflosculosis T.), vel corollulis fistulosis disci, ligulatis ambitûs (Radiati T.).

Impletio, hisce intellectis, fit in compositis, duplici modo. a. Pleni pér radium in Radiatis, dum Radius muliplicatus expellit discum floris:

Heliantbus, Calendula, Chrysantbemum. Anthemis, Matricaria, Ptarmica, Tagetes. Matricaria flore pleno, C. B.

Centaursa Cyanus.

5. Pleni per discum, cùm radius non multiplicatus, sed corollulæ dísci elongantur, et ore minùs dividuntur; quibusdam radius planus etiam fit fistulosus. Matricaria foliis florum fistulosis. H. A. P. Belli; bort. rubr. fiore multiplici fistuloso. $\mathrm{T}$. Tagetes max. rect. fiore multiplicato. Herm. lugdte. flore fistuloso duplicato. Herm. lugdb.!

Serratula Carduus in avena. fit plena corollulis elongatis, et majoribus.

28. Flores simplicium (I I5) Pleni (I II) differunt a compositis naturalibus ( 1 18), quòd pleni illi Pistillum commine in centro floris: compositi verò Stamina, et Pistilla propria obtineant.

Canon inservit Tyroni; sit e. gr.

Compositus. Flos naturalis e semiflosculis. Hieracium. Simplex Flos impletus polypetalus. Lychnis. Tum Hieracio petalis propria stamina, et pistilla: at Lychnidi Pistilli communis rudimentum adsit. Compositis ergo fructus, stamina, et pistilla singulo petalo propria simplicium veró plenis Floribus Pistillum, et Fructus' communia.

is?ib Nymphrea itaque lutea fore non est composito, nec pleno.

129. Flores compositi petalis planis plenis differunt sur. a suis non plenis, quòd Stigmata in his elongentur, et germina aucta divergant.

Canon distinguit Semiflosculosos $\mathrm{T}$. plenos a non plenis ligulatis.

Scorzonera latifolia sinuata, floribus plenis. C. B. T. Lapsana vulgaris, floribus plenis. Upsaliæ frequens. 
Tragopogon vulgare, flore pleno, 1733. Upsalix visus.

Germina cylindrica, duodecies calyce longiora, divergentia.

Pappus seminum duplo major, quàm in naturali.

Petala, et Stamina, et Stylus, ut in vulgari.

Stigmata 2, filiformia longitudine petali, longissima, non revoluta, sed variè inflexa.

130. Flores compositi pleni e petalis planis (127) differunt a compositis naturalibus (I I 7 ) petalis planis, quòd pleni illi antheris destituantur, quibus naturales gaudent.

Canon inservit pro distinctione inter Semiflosculoso: $T$. et Radiatos flore pleno; e.g. inter Hieracium, etChrysanthemum.

a. Compositi pleni fores e petalis planis fiunt ex Radiatis $T$. dum implentur, si radius discum totum occupat; ut in Chrysanthemo, Heliantio, Calendula.

b. Compositi naturales petalis planis sunt Semiflosculosi Tournef. ut Hieracium, Leontodon, Sonchus.

Hi duo flore $a . b$. facilè ludunt Tyrones.

Semifosculosi. T. Flosculi nunquam nisi hermaphroditi visi.

Radiati pleni verò nunquam visi sunt antheris instructi. Sic Tagetis fores pleni gaudent pistillis sine staminibus ad singulos flosculos; at veró Leontodon et staminibus, et pistillis.

I31. Radius in flore Composito naturali ( I I z), si pistillis instruitur, omnes quoque pleni fiores pistillis instruuntur; si verò destituitur, etiam destituuntur pleni.

Radiati $\Gamma$. sæpius ita implentur, ut radius discum excludat, tum evadunt omnes flosculi implentes similes radio naturali: e. gr. Matricaria Beilis, Chrysanthemum, Tagetes fore pleno, ad singulum fosculum, s. petalum stylo proprio instruuntur.

Helianthus, Calendula, Centaurea flore pleno, cùm discus extruditur a multiplicato radio, observes singulum petalum carere stylo, quemadmodum radius.

Cùm itaque nullus radii flosculus, in flore naturali Radiato, gaudet Antheris, sequitur etiam facillima distinctio inter Semifosculosos T. et Radiatos plenos ( 130 ). 


\section{S E X U S.}

132. Initio rerum, ex omni specie viventium (3) unicum sexûs par creatum fuisse contendimus.

Oratio nostra de Telluris habitabilis incremento. Ups. et Lugd.b.

1743. hanc Sententiam explicat.

Aqua quotannis subsidet; unde Tellus amplior evadit.

Plant $æ$ diversæ indicant altitudinem perpendicularem Terræ.

Fertilitas seminum in plantis sæpe insignis, ex una radice

unica æstate semina Zeae 2000:Inule 3000: Helianthi 4000 :

Papaveris 32000 : Nicotiane 40320.

Accedunt viviradices, perennitas, Gemmæ.

Gemmæ totidem Herbæ, ergo in una arbore, trunci vix spithami latitudinem excedente, Herbæ sæpe r 0000.

Disseminatio Naturæ stupenda est.

Aëris vis, puesertim vere, et autummo procellæ.

Esigeron 3. Hort. Cliff. 407. ex Americâ disseminata per Europam.

Fructus elevatur per caulem.

Scandentes itaque factæ, ut attcllant fructum.

Capsulæ apice dehiscunt.

Volitantia Pappo plumoso: Composite, Valeriana.

piloso: Composite, Stapelia, Xylon.

calyce: Composite, Scabiosa, Statice,

Lagecia, Brunia, Trifolium.

caudâ : Pulsatilla, Populus, Typha, Lagurus, Arundo, Siccharum.

Alâ Seminis: Abies, Liriodendrum, Betula, Plumeria, Bignonia, Conocarpus, Anethum, Artedia, Hesperis, Corispermum, Thalictrum. Pericarpii: Acer, Fraxinus, Isatis, Begonia, Hematoxylon, Ulmus, Ptelea, Dioscorea.

Calyce : Humulus, Rajania, Rumex. Inflatione, ut volumen levius evadat: Calyce : Physalis, Cucubalus, Trifolium. Pericarpio: Colutea, Fumaria, Staphylcea, Cardiospermum, Cicer. 


\section{SEXUS.}

Elasticre longè propellunt Semina.

Cartilagine ; Tricocce (ord. nat. 47.) Impatiens, Ox $x$ lis, Diosma, Dictamnus.

Mucrone: Fusticia, Ruellia, Barleria, Latbrea.

Fibris : Momordica, Cucumis, Cardamine.

Reptatu : Crupina, Avena, Geranium, Sigesbeckia, Equisetum, Filices.

Adherentia animalibus hamis, qux in ruderatis crescunt.

Calyce: Arctium, Agrimonia, Neurada, Rhexia, Asp:rugo, Rumex, Urtica, Parietaria, Plumbago, Linnaa, Sigesbeckia.

Pericarpio: Triumfetta, Bartramia, Heliocarpus, Petiveria, Triglochin, Martynia, Hedysarum, Glycyrrbiza, Scorpivirus, Vella, Circea, Valantia, Aparine.

Seminibus: Cynoglossum, Myosotis, Verbena, Daucus, Caucalis, Sanicula, Bidens, Verbesina, Arctopus.

Animalia, quæ semina integra deglutiunt, disseminant cum foenore: Viscum, Avena, Juniperus, Epidendrum.

Baccæ creatæ, ut disseminentur ob pulpam.

Sub Manducatione Semina dispergunt Sciuri, Mures, Monedulæ.

Fodiunt Talpa, Erinaceus, Lumbricus, ut terra recipiat.

Flumina, Mare, Lacus, Imbres, Astus juvant.

Anastatica exemplo mirabili, et stupendo.

Conservatio naturalis Seminum: Cassia, Mimosa, Cucumis.

Maris fundus non destruit Semina.

Similitudo eludit animalia : Salicornia, Medicago,

Plantæ occultant: Arachis, Trifolium, Latbyrus,

Valantia.

Armis ab animalibus defenduntur, appropriatis spinis, aculeis, caulibus.

Carnosæ propagantur foliis.

Arbores singulxe sunt, uti hortus circumseptus, miro naturæ consilio.

Germen, et Corculum in Semine ex Medullâ ; ergo omnis generatio continuata multiplicatio. 
33. Vegetabilia, sensatione licèt destituantur, æquè tamen, ac animalia vivere (3), probat Ortus, Nutritio, Rtas, Motus, Propulsio, Morbus, Mors, Anatomia, Organismus.

Ortus: ex Semine, vel Gemmâ.

Nutritio:ex humo tenuissimâ, Kylbel; cum aqua, et aëre. Hales. Etas : infantia, pueritia, adolescentia, virilitas, senectus; Arbores, Hedera.

MIotus: Horam diei observant flores semiflosculosi, et varii alii.

Pluviam præsagit manè Calendula.

Noctu nutat: Draba, Parthenium foliis ovatis crenatis, Trientalis.

flaccescit Impatiens, Amorpha; reflectitur Sigesbeckia, Triumfetta; clauduntur Mimose, Papilionacee, Lomentacex; componitur Tamarindus;

De die autem vigilant patentibus foliis.

Solem sequitur Reseda Luteola,et Flores semiflosculosi.

Defectus motûs ex umbrâ, aut sylvâ; hinc diversa statura Pini, aliorumque.

Propulsio: non enim datur in plantis Circulatio.

Morbus: Eestus, Sitis, Pernio, Fames, Polysarchia, Cancer, Insecta.

Mori, Oppositum vitæ est.

Anatomia: Vasa, Utriculi, Tracheæ, Cutis, Epidermis. Organismus: Vasa secretoria, Glandulæ.

34. Omne vivum ex ovo; per consequens etiam vegetabilia, quorum Semina esse Ova, docet eorum Finis, sobolem parentibus conformem producens.

Vivum omne ex ovo provenire exclamavit Harvaus.

Finis, et Essentia ovi consistit in puncto vitæ.

Semina Filicum Bobartius, Muscorum ego, Fucorum Reaumurius, Fungorum Michelius detexere ; de Majoribus dubium non est.

Generationes plantarum ex semine, et gemmâ esse coavas, docet Gemmarum consideratio, et præcocitas florescentix. 
135. Vegetabilia ex ovo ( 344 ) provenire, dictitat Ratio, et Experientia: confirmant Cotyledones.

Semina in omni plantâ adesse, nemo sanus negavit;

Polypi nec destituuntur ovis.

Radices, facilè omnes, Polypi naturam servant, uti patet ex Gemmis, Viviradicibus, Stolonibus.

Cotyledonum præsentia in omni planta prodeunte evincit semen adfuisse;

Generatio aquivoca dudum experimentis explosa est; et Cotyledonum præsentiâ optimè destruitur.

I36. Cotyledones animalium proveniunt e Vitello ovi, cui punctum vitæ innascitur; ergo Folia seminalia plantarum, quæ Corculum (86: VI.) involverunt, iidem sunt.

Cotyledones, et folia seminalia sunt synonima in plantis. Cotyledones lactiferi alunt Plumulam usque dum radices egerit, uti Placenta, s. Cotyledones in Animalibus.

Musci, et affines solis cotyledonibus destituuntur. Dissert. de Seminibus Muscorum.

137. Prolem non ab Ovo tantùm, nec a Genitura sola, sed ab utrisque simul prodire, probant Animalia Hybrida, Ratio, Anatomia.

Vermiculi seminales Leuwenhøkii non entia sunt;sunt tamen corpuscula, sed non viva per se ; interim focundant. Hybrida ex diversis:speciebus animalia; e. gr. Mulus ex Equâ, et Asino; parenti neutri exactè similis.

Anatomia: Placentæ, funiculique umbilicalis consideratio. Ratio: vitia hæreditaria, Canes, Gallinæ.

138. Ovum non fæcundatum germinare, negat omnis experientia; adeoque et Ova vegetabilium.

239. Omnis species Vegetabilium ( 157 ) flore, et fructu instruitur; etiam, ubi visus eosdem non assequitur.

Muscorum semina Nos.

Lemnie flores delineati a Valisnerio.

Fucorum flores observavit Reaumur.

Pilularice flores investigavit $B$. Jussiccius.

Fungorum stamina descripsit Micbelius. 
140. Flos (88) omnis (139) instruitur Antheris (86), et Stigmatibus (86).

Isoëtis Antheras deteximus: Iter Scanic.

Parnassice stigma deficit in flore, (postea excrescit), sed Germen hiat.

Musci Pistillis destituuntur fortè soli, cùm Corcula nuda sint.

I4r. Flos (140) antecedit omnem fructum, ut Generatio partum.

Colcbicum, et Hamamelis autumno florent, cujus fructum in sequenti anno producunt.

Muse fructus non præcedit flori, licèt maximum sit Germen, et non foecundatus cessat adolescere, non verò maturescere.

Ergo flos antecedens fructûs, et fructus semper consequens floris.

142. FRUCTIFICATIO (88) constat Plantarum Genitalibus (1 43. I 44): sic FLORESCENTIA ( I 40) est Generatio, FRUCTUS maturatio verò Partus.

143. ANTHERAS (140) esse plantarum Genitalia Masculina, et eorum POLLEN veram Genituram, docet Essentia (88), Præcedentia (14I), Situs, Tempus, Loculamenta, Castratio, Pollinis structura.

Situs: Didynamistis stamina adscendunt sub Corollæ labium superius, quò et se Pistillum flectit.

Monoëcice pleræque flores stamineos supra pistilliferos gerunt: Zea, Ricinus.

Tempus: Monoëcire, et Dioëcice flores Masculi eodem tempore Antheras, quo Pistilla Stigmata perficiunt.

Castratio: Musa oppositum, ubi flores staminei seriùs quàm pistilliferi, sterilem gerit fructum absque Seminibus. Melonis flores stamineos qui diligenter auferunt, fructus non obtinent.

Loculamenta: Anthere uniloculares, biloculares, triloculares, quadriloculares, exactè uti Pericarpium. §. Iór. Pollinis structura singularis, et determinata, ut semina. .. I or. Est omne Pollen vesiculare, et continet materiam impalpabilem, quam explodit. 


\section{$S E X U S$.}

144. STIGMATA (140) Germini ubique adnexa (97) esse Genitalia Feminina, probat Essentia(88), Præcedentia (I4), Situs, Tempus, Casus, Abscissic.

Situs: Syngenesistce rarò steriles, ubi stigmata perforant quasi antheras.

Tempus: Viget Stigma eodem tempore, quo Antheræ pollinem efflant.

Decidentia: Cadit, et marcescit Stigma post casum antherarum in plerisque; ergo effectus sub florescentiâ. Abscissio: sic Castratio in omni flore.

\section{I45. GENERATIONEM (138) Vegetabilium fieri} mediante Pollinis Antherarum illapsu supraStigmata nuda, quo rumpitur Pollen: efflatqne ciuram seminalem, quæ absorbetur ab humore Stigmatis ; confirmat Oculus, Proportio, Locus, Tempus, Pluviæ, Palmicolæ, Flores nutantes, submersi, Syngenesia, immo omnium florum genuina consideratio.

Oculus: Pollinem intrare Gernina, credidit Morilandus; ejusdem essentiam extrahi mediante stigmate madido, statuit $V$ aillant; Pollinem Aceris rumpi in humore, vidit $B$. Fussireus: omnem Pollinem in humore explodere auram seminalem, confirmavit Needham.

Proportio; Stigmata sese flectere ad antheras, dein exseri, ex Diantho, Passiforâ, Nigella patet.

Pistillum ubi brevisimum, connivent Antheræ supra stigmata: Saxifraga, Parnassia.

Connivent, dum efflant pollinem Anther in Celosia. Comprimit Corolla digitis Antheras ad Stigmata in Teucrio.

Locus: Nunquam Pistillifer $x$ : sponte nascuntur sine Staminiferis in eadem terrâ; prodeunt ex eodem semine ambx.

Tempus: in Diclinis flores ante germinationem foliorum plerumque prodeunt, ne folia tegant Pistilla: $S a-$ lix, Populus, Corylus, \&c.

Pluvice: combibunt Pollen, ut in Stigmata cadere nequeat; Hortulanis notissimum in Drupiferis, et Pomiferis. Agricolis detestabiles in agris secalinis. 
Furnus idem etiam facit, absorbendo humidum stigmatis.

Palmicole: notissima Theophrasto, Plinio, Krempfero, aliisque.

Pistacice cultura in Archipelago: Tournefortius.

Caprificatio Veterum, et adhuc in Archipelago per

Insecta. Vide Dissert. nostr. de Ficu.

Flores nutantes gaudent pistillo staminibus longiore, ut cadat

Pollen in stigma: Campanula, Lieucojum, Galanthus,

Fritillaria.

Flores submersi adscendunt sub florescentiâ: Nymphèa,

Stratiotes, Myriophyllum, Potamogeton, Hydrocharis,

Valisneria.

Syngenesia frustranea, ubi stigma deest, ibi nulla foecundatio:

in radio Centaurex, Heliantbi, Rudbeckice, Coreopsidis.

Tulipe solitariæ si auferantur antheræ ante casum Pollinis, sterilis evadet.

Brassica diversarum varietatum, sub florescentiâ in eodem loco satæ, nunqquan distincta dabunt Seminá.

Rhodiola in Horto Up's. sterilis erat ab anno 1702 . in 1750 . dum mas adducebatur, tum semina protulit.

Clutia sterilis erat in f Horris plerisque belgicis:sed visâ Leydx feminâ fuecundâ, Narem adesse prædixi, et reperi.

Cave, ne Ficus, Humulus, Musa, Morus, \&c. fructiferæ absque Staminibus dissuadeant; Distinguas Partes fructificationis, Calycem, Pericarpia, Receptacula a Seminibus.

146. CALYX ergo est Tbalamus, COROLLA Auleum, FILAMENTA $V$ asa Spermatica, ANTHERF: Testes, POLLEN Genitura, STIGMA Vulva, STYLUS Vagina, GERMEN Ovarium, PERICARPIUM Ovarium focundatum, SEMEN Ovum.

Calyx posset pro Cunni labiis, vel Proputio etiam haberi. Corolla posset etiam loco Nympharum sumi.

Filamenta, quæ succum ad antheras deferunt, vasa spermatica dicuntur.

Antheræ Testiculi sunt.

Stigma Vulva, respondens parti illi, qure in sexu sequiore lympham genitalem secernit.

Stylus Vagine, vel Tubre Fallopianre respondet, licèt huic minùs propriè. 
Germen Ovarium, cùm contineat seminum rudimenta.

Pericarpium Ovarium frecundatum, unde ova producit foecunda.

Semina Ova esse ex dictis $\$$. I 34 . I35. patet.

147. Plantarum VENTRICULUS est Terra, VASA CHYLIFERA Radix, OSSA Truncus, PULMONES Folia, COR Calor; hinc Planta Animal inversum veteribus dictum fuit.

Animal inversum olim planta dicta fuit; haurit radice, tanquam vasis lacteis, chymum e minimis particulis hu$\mathrm{mi}$, quod adscendit per caulem rigidum, in cujus divaricatione enascuntur Genitalia.

Cor plantis nullum, sed Calor efficit omne; nec opus est Corde, ubi nec perpetui mobilis effectus necessarius est, et ubi propulsio, non circulatio humorum.

Folia in motu constituta, et perspirantia hoc modo pulmonibus respondent : in se tamen re ipsâ musculi analoga sunt, licèt non uti in animalibus, caudâ affixa, cûm motus voluntarius in his dari nequeat.

148. FLOS (140), qui Antheras (143), MASCULUS; qui Stigmata (I 45), FEMINEUS; qui utraque (143. 144) continet, HERMAPHRUDITUS dicitur.

Hermaphroditi adeo frequentes sunt in Vegetabilibus, ut rariores in Animalibus; intér Vermes multi vidénțur esse hermaphroditi; Androgynæ certè Cochlece sunt. Necessaria est sexûs conjunctio in plantis, quum conjugem quærere, et adire nequeant.

«49. PLANTA, quæ floribus tantùm masculis ( 148 ), MAS quæ floribus tantüm femineis ( 148 ), FEMINA,

quæ floribus tantùm hermaphroditis

( 148$)$, HERMAPHRODITA; quæ masculis, et femineis simul, ANDROGYNA;

quæque hermaphroditis, et femineis, 
aut masculis simul, POLYGAMA dicitur: hæc tamen plerumque Hermaphrodito, aut Hermaphrodita constat.

Hermaphrodita Planta gerit super eamdem Radicem Flores omnes staminibus, pistillisque instructos, ut Genera pleraque.

ANDROGYNa gerit super eamdem radicem Flores et Masculos, et Femineos simul.

Ceratocurpus, Zannichellia, Callitriche, Cynomorium. Zea, Coix, Carex, Axyris. Typha, Sparganium, Phyllanthus, Tragia. Urtica, Alnus, Morus, Plantago,

Xantbium, Betula, Busus.

Amaranthus, Compositæ, et Umbellatæ nonnullæ. Zizania, Rumex. Myriophyllum. Ceratophyllum, Sagittaria, Poterium, Quercus, Corylus, Carpinus, Juglans, Fagus, Liquidambar.

Pinus,

Ricinus, Acalypha, Cucurbita, Bryonia, Andrachne, Fucus, Bryuin, Abies,

Fatropha, Theligonum, Cucumis, Sicyos,

Cupressus, Sterculia, Hura, Trichosanthes, Momordica, Fevillea,

Isoëtes, Hypnum,
Pilularia.

Phascum,

Mas, aut Fevina gerit Flores in eadem plantâ aut solos Masculos, aut Femineos tantùm.

$\begin{array}{llll}\text { Najas? } & & \\ \text { Valisneria, } & \text { Salix, } & \text { Rumex, } & \\ \text { Osyris, } & \text { Ficus, } & \text { Valeriana. } \\ \text { Viscum, } & \text { Hippomane, Myrica, } & \text { Hippophaë, } \\ \text { Morus, } & \text { Urtica. } & & \\ \text { Antidesma, } & \text { Rhus, } & \text { Rhamnus. , } \\ \text { Pistacia, } & \text { Ceratonia, Pisonia; } & \text { Zanonia, } \\ \text { Humulus, } & \text { Cannabis, } & \text { Acnida, } & \text { Spinacia, } \\ \text { Smilax, } & \text { Tamus, } & \text { Rajania, } & \text { Dioscorea, } \\ \text { Cissampelos, } & \text { Phonix, } & \text { Borassus. } & \\ \text { Populus, } & \text { Diospyros, Begonia, } & \end{array}$


Laurus,

Carica,

Datisca,

Aruncus,

Cliffortia.

Juniperus,

Napæa,

Ruscus.

Clutia.

Polytrictum, Mnium, Splachnum.

Cucubalus, Silene.

Taxus,

Croton.

Ephedra.

Mercurialis, Hydrocharis.

Guilandina, Kiggelaria, Coriaria.

Polygama (Hybrida aliis) constat floribus hermaphroditis necessariò, dein alios habet alterius. sexûs, et quidem modis sequentibus:

fl. Hermaphroditi, et Hermaphrodite, ut alter flos hermaphroditus sit ab altera parte, et alter a contraria sterilis, ut. Musa.。

fi. Hermaphrodite, et Masculi in eadem plantâ: Veratrum, Celtis, AEgilops, Valantia.

fl. Hermaphrodita, et Masculi in distinctâ plantâ: Chamarops, Panax, Nyssa, Diospyros.

fl. Hermaphroditi, et Feminei in eadem plantâ: Parietaria, Atriplex:

fl. Hermaphroditi, et Feminei in distinctâ plantâ:

f. Androgyni, et masculi in distinctâ plantâ: Arctopus.:

fl. Polygami ex. Hermaphrodit $\hat{n}$, et Masculo in eadem plantâ: Femina in distinctâ: Gleditsia.

fl. Hermapbroditi, Mlasculi, et Feminei in diversis plantis: Empetrum.

I50. LUXURIANTES Flores (I I9) nulli Naturales, sed omnes Monstra sunt.Pleni (I2 I) enim Eunuchi evaserunt, adeoque semper abortiunt; Multiplicati.(120) non: equè; Proliferi (122) monstrosnrum augent deformationem:

Flos perféctè plenus nullus propagatur seminibus: ergo ipse prodit vel e depactis: ramis, vel e viviradicibus; ut Diantbus, Lychnis, Hepatica, Cheirantbus, Tropaolum, Rosa, Punica, C'altha, Ranunculus, Viola, Pconia, Narcissus.

Pau-. 
Pauci Flores, ut Papaver, Nigella, potiùs multiplicatî dicendi, quàm perfectè pleni, semina perficiunt.

Luxuriantes flores multiplicant corollam cum damno staminum, qux excrescunt in petala; adeoque Multiplicati flores plurima, Pleni omnia stamina amisere. Multiplex ordo petalorum ideam præbet floris multiplicati; at verò Nymphree, Cacti, Mesembryanthemi flores multiplicati non sunt dicendi, cùm evadant tales absque damno staminum.

Sponsalia plantarma, qui de Sexa planiarun plura velit, adeat. 


\section{CHAR A C TERES.}

r5 I. FUNDAMENTUM Botanices (4) duplex est: Dispositio, et Denominatio.

Syst. nat. veget. 2. Fundamentum Botanices consistit in Plantarum Divisione, et Denominatione Systema ticâ: Genericâ, et Specificâ.

Class. plant. 4. Nomina plantarum debent esse certa, adeaque imposita certis Generibus.

Dispositio est Denominationis fundamentum:

Scientia Botanices his cardinibus nititur. Sic plantre omnes uno anno, primo intuitu, absque præceptore, sine iconibus, aut descriptionibus, constanti memoriâ addiscuntur. Ergo, qui hoc novit, Botanicus est, alius nor.

152. DISPOSITIO ( I5I $^{2}$ Vegetabilium divisiones, $s$. conjunctiones docet; estque vel Theoretica, qua Classes, Ordines, Genera; vel Practica, quie Species, et Varietates instituit.

Dispositio plantarum, ex fundamento fructificationis, recentiorum inventum est.

Practica ab eo potest tractari, quide Systemate nihil intelligit. Theoretica curam Systematis gerit; hanc Cesalpinus, Morisonus, Tournefortius, et alii excoluere.

153. Dispositio Vegetabilium (152) vel Synopticè, vel Systematicè absolvitur, et vulgò Methodus audit. Synoptica divisio seculo XVI. et XVII. maximè in usu fuit. Systematica verò seculo XVIII. præcipuè exculta fuit, incepta a Tournefortio, et Rivino.

Methodici summi methodo matbematicâ , in Scientia naturali, a simplicioribus ad composita adscendunt, adeoque incepere ab Algis, Muscis, Fungis, uti Rajus, Boerbavius EC.

Naturalis instinctus docet nosse primùm proxima, et ultimò minutissima, e. gr. Homines, Quadrupedia, Aves, Pisces, Insecta, Acaros, vel primùm majores plantas, ultimò minimos Muscos.

Natura ipsa sociat, et conjungit Lapides, et Plantas; Plan. tas, et Animalia; hoc faciendo non connectit perfectissi- 
mas Plantas cum Animalibus maximè imperfectis dictis, sed imperfecta animalia, et imperfectas Plantas combinat, e. gr.

Lerneam Animalculum, et Confervan Algam.

Spongiam Algam, et Corallia Animalia.

Taniam, Confervam articulatam, Corallinam.

Lithoceratophyton B. intra Vegetabile, extra Lapideum ex Animali.

54. SYNOPSIS ( 153 ) tradit Divisiones ( 152 ) arbitrarias, longiores aut breviores, plures aut pauciores; a Botanicis in Genere non agnoscenda. Synopsis est dichotomia arbitraria, quæ instar viæ ad Botanicem ducit, Limites autem non determinat.

Clavis Classium Synoptica est ex artis lege, ne confundantur distinguenda.

Methodici varii hanc viam incesserunt: Rajus, Knautius, abii.

I55. SYSTEMA ( I $_{53}$ ) Classes per 5 appropriata membra resolvit: Classes, Ordines, Genera, Species, Varietates.

Exempla hæc illustrant in aliis scientiis.

Geogr. Regnum, Provincia, Territorium, Parcecia, Pagus. Milit. Legio, Cohors, Manipulus, Contubernium, Miles. Phil. G. Summum, Intermed.Proximum, Species, Individ. Botan. Classis, Ordo, Genus, Species, Varietas.

Tournefortio debet Botanice hos familiarum limites. Differentia inter Synopsim, et Systema hæc est:

Synopsi : $a 2 ; b 4 ; c 8 ; d 16 ; e 32$.

Systemati: $a 10 ; b 100 ; c 1000 ; d 10000 ; e$ 100000. itaque præstat Systema Synopsi.

156. Filum ariadneum Botanices est Systema (155), sine quo Chaos est Res herbaria.

Exemplo sit planta incognita indica : evolvat Botanophilus descriptiones, figuras, indices omnes, nec reperiet nomen, nisi casu; sed Systematicus sive antiquum, sive novum genus, mox determinabit.

Systematicis, qui filum hoc duxere, omni ævo honos permanebit, quum eo destituti Mrandros Botanices errantes intrarent omnes. 
Veri Systematici Auctores, s. Inventores probè a Compilatoribus distinguendi.

Systema etiam omissas indicat per se plantas, quod nunquam Catalogi enumeratio.

157. SPECIES (155) tot numeramus, quot diversa formæ in principio sunt creatæ.

Class. plantar. 5. Species tot sunt, quot diversas formas ab initio produxit Infinitum Ens ; quæ formæ, secundùm generationis inditas leges, produxere plures, at sibi semper similes. Ergo species tot sunt, quot diversa formæ, s. structuræ hodienum occurrunt.

Oratio de Telluris babitabilis incremento, Ups. et Leyd. edita, consequentias plurimas super hoc argumentum edocuit.

Radix extenditur in herbam, inque infinitum, usque dum apice rumpantur integumenta in florem, formantque semen contiguum, ultimum terminum vegetationis. Hoc semen cadit, prognascitur, et in diverso loco quasi plantam continuat; hinc simillimam sobolem producit, uti Arbor ramum, Ramus gemmam, Gemma herbam; ergo Continuatio est Generatio plantarum.

Novas Species dari in vegetabilibus, negat generatio continuata, propagatio, observationes quotidianæ, Cotyledones.

Dubium movere Marchant. act. paris. 1719; Ego in Pelori. 1744; Gmelinus in Orat. inaugur. 1749. Vide Amanit. acad. $7 \mathrm{I}$.

Nymphoides T. Herba Nymphrex, fructificatio Menyanthis. Datisca , mas Cannabis; femina Reseda. Tragopogon Hort. ups. 3. quasi ex patre . Lapsanâ. Hyoscyamus Hort. ups. 2. . . . . . Plysalide. Poterium Hort. ups. 2. . . . . . Agrimonia. Saxifraga Fl. suec. 358. . . . . . . Parnassiâ. Dracocephalum Hort. ups. 6. . . . . . Nepetâ. Primulce sp. alpina. ............ Cortusâ. Carduus Hort. ups. I. degenerans in Carduum Pyrenaicum. Mesembryanthema numerosa ad Caput Bone Spei. Gerania africana, conformia flore, ad Cap. Bon. Spci.

Cacti omnes in sola America. Aloë numerosissimx in Africa.

Varietates singulares numerosa. Tournef. Corollar. Verbeas virginica nobis observata. 
Delphinium observante Gmelino.

Mercurialis foliis multifidis Marchantii.

I58. VARIETATES (155) tot sunt, quot differentes plantæ ex ejusdem speciei (I 57) semine sunt productæ.

Varietas est Planta mutata a causa accidentali, Climate, Solo, Calore, Ventis, \&c; reducitur itaque in Solo mutato. Species Varietatum sunt Magnitudo, Plenitudo, Crispatio, Color, Sapor, Odor.

Excludi possent varietates e Re herbaria, at

Oeconomi Magnas, et Crispas,

Hortulani Plenas, et Coloratas,

Medici Sapidas, et Odoratas æestimant.

Tropeolum flore pleno ex America curâ Bewerningii I684.

I 59. GENERA (I55) tot dicimus, quot similes constructæ fructificationes proferunt diversæ Species (I57) naturales.

Cresalp. Confusis Generibus confundi omnia necesse est.

Class. plant. 6. Omnia Genera, et Species naturales esse, confirmant revelata, inventa, observata.

Syst. nat. veget. 14. Genus omne est naturale, in primordio tale creatum; hinc pro lubitu, et secundùm cujuscumque theoriam non protervè discindendum, aut conglutinandum.

Exemplo constat Ramunculi, Aconiti, Nigellee, Claytonia, Hibisci, Passiflore, infinitorum, præsertim a posteriori.

I60. CLASSIS ( 55 ) est Generum (I 59) plurium convenientia in partibus fructificationis (86) secundùm principia naturæ, et artis.

Tournefort. Classis est congeries generum, quibus nota quædam communis adeo propria est, ut ab aliis omnibus generibus plantarum prorsùs differat.

Naturales dari classes ( $\$$. 77.) ita creatas, patet ex plurimis: Umbellatis, Verticillatis, Siliquosis, Leguminosis, Compositis, Graminibus, \&c.

Artificiales classes succedaneæ sunt naturalium, usque dum omnes naturales sint detectæ, quas plura genera, nondum detecta, revelabunt, et tum limites classium difficillimi evadant. 
Cavendum, ne imitando naturam filum ariadneum ( 156 ) amittamus, uti Morisonus, et Rajus.

r6r. ORDO ( 155 ) est Classium ( 160 ) subdivisio, ne plura Genera ( 159 ) distinguenda simul, et semel evadant, quàm animus facilè assequatur.

Ordo est Classium subdivisio ; faciliùs enim distinguuntur genera Io, quàm 100 .

62. NATURE opus semper est Species' (57), et Genus (I 59): CULTURAE sapius $V$ arietas (I 58); NATUR珢, et ARTIS Classis (16o), et Ordo (16 I). Species constantissimæ sunt, cùm earum generatio est vera continuatio.

Genera esse naturalia, evincunt plurimæ plantæ: Aconita, Nigalle, Bignonic, Ranunculi, Mesembryantbema, Zyge: phylla, Gerania, Oxalides.

$V$ arietates culturæ opus esse, docet Horticultura, quæ easdem sæpius et producit, et reducit.

Classis, et Crdines plerosque naturales esse, docent Ordines naturales $\$ .77$.

163. HABITUS est conformitas quædam Vegetabilium affinium, et congenerum, in Placentatione, Radicaicone, Ramificatione, Intorsione, Gemmatione, Foliatione, Stipulatione, Pubescentia, Glandulatione, Lactesce, itia, Inflorescentia, aliis. que.

FACIES EXTERNA vulgò a Botanicis antea dictus est Habitus.

C. Baubinus, et $V$ eteres egregiè ex Habitu plantarum divinarunt earumdem affinitates, ut ipsi Systematici sæpius deflexerint, ubi Habitus rectâ duxerat.

Methodus naturalis est ultimus finis Botanices (\$. 77.)

Fructificatio, Recentiorum inventum, viam primario aperuit ad Methodum naturalem, sed nec hæc etiamnum ita intelligitur, ut,omres classes detegat.

Habitus, uti in Quadrupedibus distinguit Feras a Pecoribus, quamvis Dentes non inspicerentur; sic etiam in Plantis sæpe harum ordines naturales primo intuitu manifestat. G 3 PLA - 
I. PLACENTATIO est cotyledonum dispositio, sub ipsa Seminis Germinatione.

I. ACOTYLEDONES, ubi nulli omnino exstant cotyledones: Musci.

2. MONOCOTRLEDONES (quamvis hæ propriè acotyledones sint, cùm cotyledones persistunt intra Semen.)

perforatæ : Gramina.

unilaterales: Palme.

reductæ: Cepa.

3. DICOTYLEDONES immutatæ: Legumina, Poma, Drupe, Didynamia.

plicatæ: Gossypium.

duplicatæ: Tetradynamia, Malve.

obvolutæ: Helsine.

spirales : Salsola, Salicornia, Ceratocarpus, Basella, Oleracere omnes:

$77: 52$.

reductæ: Umbellate.

4. POLTCOTTLEDONES Pinus Io.

\section{Cupressus 5. \\ Linum 4?}

II. RADICATIO est Radicis dispositio: caudice descendente, adscendente, et Radiculis. Exempla. ex \$. 80 . petenda.

Bulbosa squamosa : Lilium; Tunicata: Cepa;

Duplicata: Fritillaria; Solida: Tulipa.

Tuberosa palmata: Orchis; Fasciculata : Pæonia.

Pendula: Filipendula, Eleagnus.

Articulata (85): Lathrea, Oxalis, MIartynia, Dentarid.

Fusiformis: Pastinaca, Dinucus, Raphanus.

Globosa: Ranunculi spec. Chorophylli sp. Bunium.

1II. RAMIFICATIO manifestatur in situ Ramorum, quem Folia sequuntur.

Nulli Rami, licèt folia prodeant in caule: Dictamnus, Pronia, Epimedium, Podophyllum.

Folia opposita, et alterna in plantis, plerumque vegetabilia diversissima indicant, paucas si excipias, quarum aliæ species foliis oppositis, aliæalternis sunt: ut

Euphorbia, Cistus, Lantana. Antirrbinum, Lilium, Epilobium. 
Folia infernè opposita ad ramos, supernè alterna ad flores: Antirrbinum, fasminum.

Veronica, Borrago.

Folia infernè alterna, superne opposita in ramos: Potentilla I. H. upsal. Potamngeton.

Folia infernè opposita, supernè terna : Nerium.

Folia infernè terna, supernè alterna (rami infernè terni, nec alii) : Ruscus.

Folia infernè quaterna, supernè alterna: Coreopsis 2.Hort.ups. Antirrbinum 3. Hort. ups.

Naturalem situm qui eruere gestit in plantis ramificatione diversis, adeat Folia radicalia.

IV. INTORSIO est "flexio partium versùs alterum latus.

CAULIS volubilis sinistrorsùm $\mathbb{C}$ :

Tamus, Dioscorea, Rajania, Menispermum. Cissampelos, Hippocratea.

Lonicera.

Humulus.

Helxine. dextrorsùm $\Phi$ :

Phaseolus, Dolichos, Clitoria, Glycine, Securidaca.

Convolvulus, Ipomea,

Cynanche, Periploca, Ceropegia,

Euphorbia, Tragia.

Basella,

Eupatoriuin,

Tournefortia.

CIRRHUS volubilis dextrorsum, retrorsumque.

Leguminosa pleræque ejusmodi cirrhos gerunt.

Smilax petiolos cirrhiferos profert; idem ferme Piper. COROLLA sinistrorsum (*):

Asclepias, Nerium, Vinca, Rauzvolfia, Periploca; Stapelia.

dextrorsum: Pedicularis Fl. su. 505. 507. 508.

(*) Sinistrorsum hoc est, quod respicit dextram, si ponas Te ipsum in centro constitutum, meridiem aspicere; Dexirorsum itaque contrarium. 
Trientalis singularis, quòd petala omnia altero latere imbricatim dextrorsum in. cumbant.

Gentiana contra solem, ante explicationem, imbricata est.

- PISTILLA sinistrorsum: Cucubalus, Silene.

GERMINA contorta sinistrorsum : Helicteres, Ulmaria.

- FLOKUMI resupinatio, cùm corollæ labium superius ferram, inferius cælum spectat: Viole europere, Ocymun, Ajuga orientalis, Satyrii species. obliquitas: Hyssopi Lopbantbus D. Nepeta H. ups. 3 . Pedicularis $₫ F l$. suec. 505,507,508.

SPICAE spirales: Asperifolic.

Claytonia.

incurvatio: Saururus, Mimosa, Petiveria, Papaver,

Sedum rubrum, Lilium martagon.

IITGROMETRICA varia ex fibrarum contorsione occurrunt in variis plantis.

Avene arista funis instar contorta.

Geranii seminis arillus caudatus spiralis.

Mnium, quod Bryum Fl.suec.903.pedunculo infernè, et supernè contrario torto.

V. GEMMATIO est Gemmæ constructio ex foliis, stipulis, petiolis, aut squamis.

1. OPPOSITIV AE PETIOLARES.

a. Ligustrum

P.hillyrea

Nyctanthes

Syringa

Hypericum

Coriaria

Busus.

b. Fasminum

Vaccinium

Arbutus

Andromede

Ledum

Dapbne
Laurus

Myrica.

c. Linnea

Diervilla

Lonicera.

d. Evonymus.

e. Fraxinus Acer

Asculus

Bignonia.

f. Opulus

Sambucus.

g. Psidium. 
3. OPPOSITIE FE STIPULACE ZE.

Cephalanthus.

Rhamnus catbarticus.

3. ALTERNATIVAE PETIOLARES.
a. Salis.
- Hippophaë.
f. Juglans.
b. Spirex.
d. Berberis
Pistacia.
c. Genista.
Solanum
Ilex:
e. Ribes.
g. Plumbago.

4. ALTERNATIV STIPULACEO-PETIOLARES. I
a. Sorbus
Crategus
Prunus.
c. Cotoneaster
Rulus?
Mespilus Germ.
b. Pyrus
Amygdalus
Vitis.
Cercsus . IJ Robinia.
Padus.
Eytisus.
Maius.
d. Meliantbus
Rosa
e. Potentilla fruticos.
Stapbylea.

5. ALTERNATIVAE STIPULACER.
a. Popuins.
Fagus
b. Tilia
Carpinus
Ulmus
Corylus.
Quercus.
c. Betula Alnus.
d. Ficus. Morus.

6. ANOMALA.
a. Abies.
b. Pinus.
c. Taxusa

7. NULLAE.

Carentes gemmis.

\$. $85 \cdot p \cdot 5 \cdot 1$.

VI. FOLIATIO est complicatio ea, quam servant Folia, dum intra Gemmam, aut Asparagos plantarum latent. Hæc prætervisa antecessoribus sequentes modos agnoscit. Tab. X.

1. INVOLUTA, quórum margines laterales utrinque introrsum spiralite involvuntur. Fig. 2. ro. I I.

2. REVOLUTA, quorum margines laterales utrinque retrorsum spiraliter convolvuntur. Fig. 3. 12.

3. OBVOLUTA, cùn margines alterni comprehendunt oppositi folii marginem rectum. Fig. 7 .

4. CONVOLUTA, cùm unius lateris margo circumambit alterum ejusdem folii marginem instar cuculli. Fig. I. 9. 5. IMBRICATA, quando parallelè, superficie rectâ, sibi invicem incumbunt. Fig. 5.

- w. EQUI- 
6. EQUITANTIA, cùm folii latera parallelè connivent, - ut interiora ab exterioribus includantur; quod non in Conduplicatis (7) obtinet. Fig. 5. I3. I 4 .

7. CONDUPLICATA; ; cùm folii latera parallelè sibi inn-

.2 vicem approximantur. Fig. 4.

8. PLICATA, cùm plicatim longitudinaliter complicantur, uti folia plicata \$. 83. Fig. 8.

9. RECLINATA, cùm folia versùs petiolum deorsum reflexa sunt.

10. CIRCINALIA, cùm folia deorsum spiraliter involvuntur.

I. INVOLUTA.

Lonicera

Diervilla.

Euonymus

Rhamnus cathart.

Pyrus

Malus ..

Populus

Plumbago

Viola

Commelina ann.

- Plantago 10 '

- Alismas

Patamogeton natans

Nymphrea

Saururus

Aster ann.

Humulus

Urtica

Hepatica

Sambucus

Ebulus

Staphylica.

2. REVOLUTA.

Rosmarinus

Teucriam Marun.

Dracoceph. digit.

Nérium

Andromeda
Ledum

Epilobium irregul.

Salicis species!

Rumex

Persicaria

- Polygonum

Parietaria

Primula

Carduus

Cnicus

Tussilago

Senecio

Otbonna

Potentilla fruticosa.

Ptelea.

3. OBVOLUTA.

Dianthus

Lychnis

Saponaria

Epilobium oppositif.

Dipsacus.

Scabiosa

Valerians

Marrubium

Phlomis

Salvia

Prasium.

4. CONVOLUTA.

Canna 
Amomum.

Calla

Arum

Piper

Hydrocharis.

Commelina lutea.

Gramina pleraque

Prunus

Armeniaca

Dodecatbeon

Crepis

Lactuca

Hieracium

Sonchus Sibir.

Tragopogon

Orobus.

Vicia

Latbyrus

Solidago Aster

Pinguicula

Vaccinium

Pyrola

Berberis

Brassica Armoracia

Symphytum Cynoglossum

Potamig. perf.

Eryngium

Menyanthes conjunctim

Saxifraga

Aralia

Dictamnus

Epimedium.

5. IMBRICATA.

Syringa

Ligustrum

Pbillyrea

Nyctanthes:

Linnea.
Cephalanthus

Coriuria

Hyperïum

Valanita

Fusticia

Portulitea

Lauris

Daphne

Hippophaë

Ruscus ...th

Cyanus pérennis

Mespilus Germ.

Campanula

Polemonium

Sium:

6. EQUITANTIA.

Hemerocallis

Gramina nonnulla.

Poa.

Iris

Acorus

Carex.

7. CONDUPLICATA. Quercus

Fagus (intimum)

Corylus semi (secundè) Carpinus (secundè) Tilia (secundè) $P$ adus spiraliter

Cerasus - - Amygdalus - -

Cotoneaster - -

Frangula Alaternus Paliurus

Fuglans.

Pistäcia

Rhus

Fraxinus

Sorbus

Rosa 


\begin{tabular}{|c|c|}
\hline Rubus & \\
\hline Potentilla vulg. & Viburram \\
\hline Comarum obvolutum! & Ribes : \\
\hline Bignonia & Althea \\
\hline Cytisus & Malva \\
\hline Robinial & Humulus \\
\hline Pisum & : Urti \\
\hline Diadelphic plercequ & Passifiora \\
\hline Melianthus & Alchemilla. \\
\hline Pastina & 9. RECLINATA. \\
\hline Her & Podo \\
\hline Lase & Acon \\
\hline Poteriu & Hepatica \\
\hline PLIC & Pulsatilla \\
\hline Crutregus & Anemone \\
\hline $\begin{array}{l}\text { Betuld } \\
\text { Alnus }\end{array}$ & $\begin{array}{l}\text { Adoxa. } \\
\text { D. CIR CINALI }\end{array}$ \\
\hline Fagus! in & Filices \\
\hline $\begin{array}{l}\text { Vitis } \\
\text { Acer }\end{array}$ & Palme nonnulle. \\
\hline
\end{tabular}

VII. STIPULATIO est stipularum situs, et structura ad basim foliorum.

Stipulæ non minùs, quàm folia in diversis diversæ existunt.

a. Nullæ: Asperifolice, Didynamia, Stellate, Siliquose, Liliacee, Orchidere, Composite plurime.

Præsentes: Papilionacex, Lomentacer, Icosandria.

b. Geminx, s. utrinque solitarix: Plerisque.

Solitariæ: Meliantbus internè ; Ruscus externè.

c. Decidur: Padus, Cerasus, Amygialus, et ordo V. Gemimarum.

Persistentes: Diadelphia, Icosandria polygynia.

d. Adnatæ: Rosa, Rubus, Potentilla, Comarum, Meliantbus. Solutæ: Plerisque.

e. Intrafoliacex: Ficus, Morus.

Fxtrafoliacex: Diadelpbia, Alnus, Betula, Tilia.

VIII. PUBESCENTIA est Armatura plantæ, qua ab externis injuriis defenditur.

SCABRITIES componitur particulis, nudis oculis vix manifestis, quibus adspergitur plantarum superficies. 
Guetrardus lynceis oculis hanc, quasi primus, vidit. Glandulosa: Niliaris.

Vesicularis, Mesembryanthemum, Aizoon, Tetra-

Lenticularis. gonia.

Glcbularis, Atriplex, Chenopodium.

Secretoria.

Catenulata.

Utricularis.

Setacea : Cylindrica.

Conica.

Hamosa.

Glandulifera, Ribes.

Furcata, Lavandula.

Securiformis, Humulus.

Aggregatæ stellares, Alyssum, Helicteres.

Articulata simplex. simplices, Hippophä.

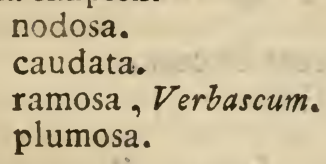

LANA servat plantas ab æstu nimio.

Salvia canariensis : Sideritis canariensis, Salvia cethiopis.

Marrubium, Verbascum, Stachys.

Carduus eriocephalus, Onopordum.

TOMENTUM servat plantas a ventis; gaudet sæpius colore incuno:

Tomex.

Medicago, Halimus.

STRIGE压 arcent setis rigidis animalcula, et linguas:

Cactus.

Malpighia.

Hibiscus, Rubus.

HAMI adhærent animalibus prætereuntibus.

Triglochides: Lappula.

Incurvi : Arctium, Marrubium,

Xanthium, Petiveria. 
STIMULI puncturâ venenatâ arcent animalia nuda.

Urtica,

Fatropha, Acalypisa, Tragia.

ACULEI arcent propria animalia.

Volkameria, Pisonia.

Hugonia (spirales, s. cirrhosi).

Cresalpinia, Mimosa, Parkinsonia.

Capparis, Erythrina, Robinia.

Solanum, Cleome.

Smilax, Convolvulus, Aralia.

Duranta, Xylon, Drypis.

Euphorbia, Tragacantha, Tragopogom

FURC㤅 arcent animalia.

Berberis, Ribes, Gleảitsia.

Mesembryanthemum, Osteospermum.

Ballota, Barleria, Fagonia, Poterium.

\section{SPINE RAMORUM arcent Pecora.}

Pyrus, Prunus, Citrus.

Hippophaë.

Gmelina, Rbamnus, Lycium, Catesber, Colastrus.

Ulex, Asparaigus.

Spartium, Achyronia, Ximeria.

Ononis, Stacbys, Alyssum, Cichorium.

\section{FOLIORUM.}

Aloë, Agave, rucca.

llex, Hippomane, Theophrasta.

Carlina, Cynara, Onopordum,

Morina, Acantbus, Gundelia, Zuniperus, Salsola, Polygala.

Ruscus, Borbonia, Statice, Ovieda.

Cliffortia.

CALYCIS.

Carduus, Cnicus, Centaurea.

Molucella, Galeopsis.

FRUCTUS.

Trapa, Tribulus, Murex.

Spinacia, Agrimonia, Datura. 


\section{GLANDULATIO vasa secretoria offert.}

\section{I, GLANDULFE PETIOLARES:}

Ricinus, fatropha, Passiflora, Cassia, Mimosa. FOLIACEAE ex serraturis : Salix.

ex basi: Amygdalus, Cucurbita, Elceocarpus, Impatiens, Padus, Opulus.

ex dorso: Urena, Tamarix, Croton.

ex superficie: Pinguicula, Drosera.

STIPULARES : Baubinia, Arnieniaca.

CAPILLARES: Ribes, Antirrbinum quadrifol. Scrophularia, Cerastium, Silene.

PORI: Tamarix: Silene, viscaria.

2. FOLLICULI sunt vasa aëre distenta.

Utricularia, ad radicem profert vascula subrotunda inflata bicornia.

Aldrovanda, ad folia promit folliculos olliformes, semicirculares.

3. UTRICULI sunt vascula repleta liquore secreto.

Nepenthis extremitas foliorum desinit in filum, et filum

in cylindrum magnitudine, et figurâ penis, apice clausum operculo, quod margine altero aperitur. Sarracenia folia habet cucullata, ferme uti Nepenthes, sed ad radicem sessilia.

Marcgravia in centro umbellæ promit vascula, quæ referunt corollam ringentem Galeopsidis, sed labio inferiori destitutam, Gen. plant. 507.

X. LACTESCENTIA est copia liquoris, qui effluit læsa planta.

Alba : Euphorbia.

Papaver.

Asclepias, Apocynum, Cynancbum \&c.

Semiflosculosi $T$.

Campanula, Lobelia , Fasione.

Cactus tuberculis tectus. Acer, Sclinum, Rhus. 
Lutea : Chelidonium, Bocconia, Sanguinaria, Cambogia.

Rubra : Rumex sanguineus.

XI. INFLORESCENTIA est modus, quo flores pedunculo plantæ adnectuntur, quem Modum Florendi dixere antecessores.

Verticillate Raj. Murrubium.

COR YMBIFER E: Siliquose (Ord. nat. 57).

SPICATE: (Ord. nat. r. 2.) Piperes, Mimose.

Paniculate: Grominul varia.

Axillares sunt plerique flores: ergo sequentes rariores:

Oppositifoli , quorum flores folio e regione oppo-nuntur, uti Piper, Saururus, Phytolacca, Dulcamara, Vitis, Cisti annui, Cissus, Corchorus, Ranunculus aquatilis, Geranium.

INTERFOLIACEI Flores inter folia opposita, sed alternatim collocantur: Aselepias.

LATERIFolin Flores ad latus baseos folii: Claytonia, Solanum, Asperifolice.

Petrolares Flores pedunculo petiolis inserto: Hibiscus, Turnera.

Cirrhiferi Flores: Cardiospermum, Vitis.

Supraaxillares : Asperifolice, Potentilla 3. H. ups.

Mittimus varia, quæ huc spectant, e. gr.

TEMIPUS Germinandi antequam e Semine, et Terrå emergat:

Brevissimum: Tetradynamia, Helsine.

Annuum: Hypecoum, Glaucium, Melampyrum segetum, Ranunculus falcatus.

Bienne: Mespilus, Oxyacantha, Rosa, Cornus. Grmmandi, seu gemmas aperiendi.

Florendi annuum:

\section{horarium.}

Vigendi, quod in variis, et diversis proprium, et singulare est.

I64. Dispositio ( 155 ) vegetabilium primaria ( 152 ) a solâ fructificatione desumenda est.

Antecesores urgebant insufficientiam fructificationis, quum partes ejus paucre ipsis innotuere; nos easdem sufficientissimas introduximus. 
Calyx.

Perianthium Riv.

Involucrum Arted.

Amentum.

Spatha Linn.

Gluma Raj.

Calyptra Dill.

Volva Michel.

Corolla Linn.

Petalum Col.

Tubus, s. unguis.

Limbus, s. LaminaL.

Nectarium Linn.

Stamen.

Filamentum Linn. Anthera $L$.

Pistillum.

Germen $L$.

Stylus Boerh.

Stigma Linn.

Pericarpium Riv.

Capsula Veter.

Siliqua Veter.

Legumen Linn.

Conceptaculum $L$.

Drupa Linn.

Pomum Veter.

Bacca Veter.

Strobilus $R$.

SEMEN.

Coronula $\boldsymbol{L}$.

Pappus Veter.

Ala.

Arillus $\boldsymbol{L}$.

Hilum.

Nux Veter.

Propago Linn.

Reciptaculum Pont.

Palea Vaill.

Umbella Veter.

Cyma Linn.

Spadix Linn.
Calyx Tournef.

Fulus $T$.

Locusta Raj.

Petalum $T$.

Unguis $T$.

Stamen $T$.

Apex $T$.

Ovaritum $B$.

Pistillum T. Tuba $V$.

Capsula $T$.

Siliqua $T$.

Fructus carnosus.

Conus $T$.

Calyptra $T$.

Nux $T$.

Placenta $V$.

Umbella $T$. 
Genera ficta itaque vocanda sunt ea omnia, quæ fundamentum in solâ fructificatione non agnoscunt: e. gr.

Limodorum T. Radice fibrosâ non esset Orchis. Bistorta T. Radice carnosâ ... non Polygonum. Rapa T. Radice gibbâ ...... Brassica. Sisarum T. Radice tuberosâ. ..... Sium. Hermodactylus T. Radice tuberosâ . . Iris. Sisyrinchium T. Bulbo superimposito . . Iris. Xiphium T. Bulbo tunicato ....... Iris. Lilio-Fritillaria B. Bulbo squamoso . Fritillaria. Nesomora R. Caule berbaceo .... Cornus. Anacampseros T. Caule erecto .... Sedum. Psyllium T. Caule ramoso ....... Plantago. Bellis Leucanthemum M. Caule folioso. Bellis. Pilosella V. Scapo nudo ....... Hieracium. Suber T. Cortice fungoso ...... Quercus. Larix T. Foliis fasciculatis ..... Abies. Genistella T. Foliis articulatis ... Genista. Potamopithys B. Foliis non stellatis . Alsinastrum. T. Quinquefolium T. Foliis digitatis . . Pentaphylloides T. Lupinaster B. Foliis digitatis .... Trifolium. Dracunculus T. Foliis pedatis ..... Arum. Trichomanes T. Foliis pinnatis ... Asplenium. Clymenum T. Foliis pinnatis ..... Lathyrus. Niuscoides M. Foliis imbricatis pluries . Jungermannia. Lentiscus T. Foliis sine impari ... Terebinthus T. Faba T. Foliis sine cirrbo...... Vicia. Cytiso-Genista T. Foliis ternis, et simpl. Spartium L. Colocasia B. Foliis non auritis .... Arum. Cirsium T. Foliis non spinosis ..... Carduus Coronopus R. Foliis pinnatifidis . . Cochlearia. Coronopus T. Foliis dentatis ..... Plantago. Ilex T. Foliis denticulatis ...... Quercus. Scorzoneroides V. Foliis dentatis ... Scorzonera. Anguria T. Foliis multifidis. .... Cucurbita. Alcea T. Foliis multifidis ...... Malva. Millefolium T. Foliis sectis minutim . Ptarmica $\mathbf{T}$. Cicutaria T. Foliis Cicute ..... Ligusticum. Cedrus T. Foliis cupressinis ..... Juniperus. Ranunculoides Foliis capillaribus ... Ranunculus. Alhagi T. Foliis simplicibus .... Hedysarum. 
Nissolia T. Foliis simplicibus ..... Lathyrus.

Marsilea M. Foliis simplicibus ... Jungermannia.

Balsamita V. Foliis indivisis ..... Tanacetum.

Cepa T. Foliis fistulssis ....... Allium.

A phaca T. Foliis nullis preeter stipulas . Lathyrus.

Mimosa T. Foliis sensitivis ...... Acacia T.

Oxyoides G. Foliis sensitivis pinnatis . Oxalis.

Aurantium T. Petiolis cordatis.... Citrus.

Calamintha T. Pedunculis ramosis . Melissa.

Cotinus T. Pedunculs lanatis .... Rhus.

Virga sanguinea D. Cyma nuda... Cornus.

Corona imperialis T. Coma foliosa .. Fritillaria.

Stæchas T. Spicâ comosâ. . . . . Lavandula.

Carex D. Spiculis androgynis ...... Cyperoides T.

Chamrpithys T. Floribus sparsis . Teucrium.

Acinos D. Florilus sparsis ..... Thymus.

Limonium T. Floribus sparsis .... Statice.

Chamædrys $\mathrm{T}$. Floribus verticillatis. Teucrium.

Thymbra T. Floribus verticillatis ... Satureja.

Volubilis D. Floribus capitatis .... Iponœa.

Polium T. Floribus cymosis ...... Teucrium.

Castanea T. Floribus spicatis ..... Fagus.

Fagopyrum $\Gamma$. Fl. spicatis, Rad.fibrosâ . Polygonum.

Majorana T.Flor.spicatis rotundioribus . Origanum.

GENERA FICTA ex facie rejicienda sunt omnia, nisi simul

fructificationis principio inædificata: e. gr.

Malus T. Facie propriâ itaque non. Pyrus.

Cydonia T. Facie propriâ...... Pyrus.

Armeniaca T. Facie propria ...... Prunus.

Cerasus T. Facie propriâ...... Prunus.

Lauro-Cerasus T. Facie propriâ .. Prunus.

Limon T. Facie propriâ ...... Citrus.

Napus T. Facie propria ...... Brassica.

Absinthium T. Facie externâ ..... Artemisia.

Abrotanum T. Facie externâ ..... Artemisia.

Bellidiastrum M. Habitu proprio . . . D Doronicum.

Euphorbia T. Habitu aphyllo .... Tithymalus T.

Usnea D. Habitu capillari ...... Lichen.

Coralloides D. Habitu caulescente ... Lichen.

Clavaria V. Habitu non famoso ... Coralloides $\mathrm{T}$.

Tuber T. Substantia solidiore . . . . Lycoperdon T.

Fungoides M. Substantiâ utrinque lrevi . Elvela. 
Lycoperdoides M. Substantiâ cellulari . Lycoperdon. Amanita D. Yileo stipitata ...... Agaricus. Fhallus M. Stipite basi volvato ... Boletus M. Phalloboletus Ni. Pileo lateribus libero. Boletus M. Polyporus M. Poris non distinguenüis . Boletus L. Erinaceus Mi. Aculeis densis..... Ulex. Thysselinum T. Succo lacteo ..... Selinum. Moly B. Odore suavi ......... Allium. Acetosa T. Supore acido ....... Lapaihum. T. Colocynthis T. Fruciu amaro .... Anguria. T.

165. Quæcumque vegetabilia in fructificationis partibus (86) conveniunt, non sunt, ceteris paribus (162), in Dispositione Theoreticâ ( 152$)$ distinguënda.

Gesrerus invenit, Cesalpinus introduxit, Morisonus resuscitavit, Tourneforitus enutrivit hoc summum in Botanices scientiâ inventum.

Rivinus: Quxcumque conveniunt fore, et semine, eodem nomine designandx sunt; et vi oppositorum.

Knautius : Quæcumque plantæ ad eumdem modum florent, et capsulas seminales producunt, illæ ad idem genus pertinent; et vicissim e contrario.

166. Quxcumque vegetabilia in fructificationis partibus (86) differunt, observatis observandis (162), non sunt combinanda.

Cảon ex opposito antecedentis fluit.

167. NOTA CHARACTERISTICA (I 89) omnis erui debet a Numero, Figurâ, Proportione, et Situ omnium partium Fructificationis (86) differentium (98-104).

Methodum, qua character omnis genericus conficiendus est, tradit.

Numerus non excedit 24 literas alphabeti.

Sint Fructificationis partes VII.

Calycis ........7.

Corollse...........

Staminum........

Piștilli.......... 
Partium omnium Summa 38.

$$
\begin{aligned}
& \text { Pericarpii - - } 8 . \\
& \text { Seminis - }-04 \\
& \text { Receptaculi - - } 4 .
\end{aligned}
$$

Figura

Situs

Proportio.

ergo quater triginta octo: 152.

Mutetur modus secundùm partes 38. fit 5776 . adeoque sufficit fructificatio ad minimum generibus $577^{\frac{6}{6}}$, quæ nunquam existant.

Falsò itaque adsumitur habitus ( $($ I 163 ), color, magnitudo, cotyledones, et alia, exceptis datis.

Knautius ex modo florendi plura nova genera spuria fabricavit.

Sic falsò Psyllii habitus.

Blattaria glabrities.

Numularia modus florendi.

Daphnes $K n$. modus florendi.

Cracce modus florendi multiflorus.

Limonii T. Flores sparsi.

Inula ab Astere distincta genera ex colore $V$ aill,

Cactus, Melocactus monocotyledonis, Opuntia dicotyledonis.

Cupressus Europea dicotyledonis, Americana pentacotyledonis.

168. Habitus ( $16_{3}$ ) occultè consulendus est, ne genus erroneum levi de causâ fingatur.

Experientia rerum magistra, primò intuitu, ex facie externâ plantarum familias sæpius divinat.

Exempla confirmant regulam:

Isopyrum, Nigella, Helleborus, Caltha diversa.

Sambucus, et Ebulus; Trifolium, et Triphylloides conjungenda.

Primo intuitu distinguit sæpius exercitatusBotanicus plantas Africæ, Asiæ, Americ $x$, Alpiumque; sed non facilè dicere $\mathrm{H}_{3}$ 
ipse, ex qua notâ: Nescio, qux facies torva, sicca, obscura AFRIs; quæ superba, exaltata Asinticis; qux leta, glabra AnericANIs; qux coarctata, indurata AlpiNis? Occultè consulendus est habitus, ne intret cohortem notarum characteristicarum, et genera disterminet; Exemplis probatur Boerbaavii Ind. Lugduno-Batavi; cùm nullus ex facie descriptâ homines singulos nosse studeret.

Characteres habituales sufficientes quamvis non sint, tamen primo intuitu plantam sæpius manifestant; hi confici possent sequenti modo:

CARTOPHTLLEI, Ord. nat. 42.

Placentatio dicotyledonis.

Radicatio fibrosa.

Ramificatio opposita, articulata, erecta.

Intorsio pistilli sinistra.

Foliatio obvoluta, lanceolata, indivisa.

Stipulatio nulla.

Pubescentia vix notabilis.

Inflorescentia dichotoma.

$V E R T I C I L L A T E$, Ord. nat. 58.

Placentatio dicotyledonis.

Radicatio fibrosa.

Ramificatio opposita, tetragona.

Foliatio obvoluta, simplex..

Stipulatio nulla.

Pubescentia subvillosa.

Inflorescentia verticillata, bracteata. ASPERIFOLIAE, Ord. nat. 43.

Placentatio dicotyledonis.

Radicatio fibrosa.

Ramificatio alterna.

Foliatio convoluta, simplex, indivisa.

Stipulatio nulla.

Pubescentia scaúra.

Intorsio spiralis spice recurve.

Inflorescentia laterifolia.

UMBELLATEE, Ord. nat. 22.

Placentatio dicotyledonis reducta.

Radicatio fusiformis.

Ramificatio alterna, teretiuscula, erecta.

Foliatio duplicata, supradecomposita.

Stipulatio nulla, vaginans petiolo. 
Pubescentia inermis.

Inflorescentia umbellata.

LEGUMINOS ZE, Ord. nat. 55.

Placentatio dicotyledonis immutata oblique insertx.

Radicatio fibrosa, subnodosa.

Ramificatio alterna.

Foliatio conduplicata, s. convoluta, pinnata.

Stipulatio insignis.

Pubescentia _. - -

Intorsio cirrhosa.

Inflorescentia subspicata, secunda.

SILIQUOS AE, Ord. nat. 57.

Radicatio carnoso-fibrosa.

Ramificatio alterna.

Foliatio convoluta, sublyrata.

Stipulatio nulla.

Pubescentia inermis.

Inflorescentia corymbosa, terminalis, ebracteatn. COLUMNIFERE, Ord. nat. 34 .

Placentatio dicotyledonis, cordata, subplicata.

Radicatio fibrosa.

Ramificatio alterna.

Foliatio plicata, simplex.

Stipulatio angusta, patula.

Pubescentia subtomentosa.

Inflorescentia axillaris, pedunculata.

FILICES, Ord. nat. 64.

Radicatio fibrosa, fascicularis.

Ramificatio disticha.

Intorsio circinalis.

Foliatio circinalis, pinnata.

Stipulatio nulla.

Pubescentia strigosa.

Inflorescentia dorsalis, sessilis.

I69. Quæ ( $\left.16_{7}\right)$ in uno genere ad Genus stabiliendum valent, minimè idem in altero necessariò præstant.

Scias Characterem non constituere Genus, sed Genus Characterem.*

Characterem fluere e Genere, non Genus e Charactere.* 
Characterem non esse, ut Genus fiat, sed ut Genus noscatur. *

Exempla demonstrant hoc evidentissimè :

CARIC Mas monopetalus, Femina pentapetala est.

JATroph flores Masculi monopetali, Feminei pentapetali.

Mirrica altera semine nudo, altera baccato.

Fraxinus alia flore mudo, alia corollato.

Geranium aliud corollầ regulari, aliud irregulari.

LinUm aliud pentapetalum, aliud tetrapetalum.

Aconitum aliud tricapsulare; aliud quinquecapsulare.

Controversia inde Rivini cum Dillenio (\$. 21 ).

Hæresis inde summa Botanices, quæ genuit genera spuria innumera, in sunmum damnum Botanices.

TRIFOLIUM e. gr. aliud monofetalum, aliud tetrapetalum; aliud monospermum, aliud polyspermum; urgent nonnul1i Monopetalum, et Polypetalum, Nonospermum, et Polyspermum sub eodem genere militare non posse; ergo contra Naturam genera spuria condunt.

I70. Rarò observatur Genus, in quo pars ( 167 ) aliqua fructificationis non aberrat.

Gener ficta a speciebus, aliquâ parte fructificationis diversis, plurima orta sunt: e. gr.

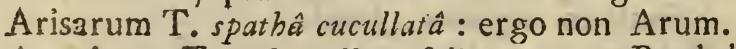

Asteriscus $T$. cal. stellato folioso - - Buphthalmum.

Silybum.V. calycis spinis - - - - Carduus.

Moldavica T. calyce gibbo bilabiato - Dracocephalum.

Tithymaloides T.calyce gibboirregulari- Euphorbia.

Trionum L. calyce inflato - - - - Hibiscus.

Ficaria D. calyce 3 pbyillo polypetalo - Ranunculus.

Iva D. calyce gibbo _. - . - . Teucrium.

Lunularia M. calyce comm. 4fido - Marchantia.

LeucanthemumT.calycis squamis angustis. Chrysanthemum.

Cardiaca T. ealyce sdentato - . - - Leonurus.

Paronychia T. calycis foliis cucullatis - Herniaria.

PseudodictamnusT.calycibus infundibul. Marrubium.

- Anemone-Ranunculus D. corollâ. spetala Anemonoides.

Linaria T. corollâ caudatâ _. - Antirrhinum.

Valerianoides V. corollâ caudatâ - - Valeriana.

Bromelia P. corollâ 3petalâ _. - - Ananas. Tourn.

Opuntia T. corolla polypetala - - - Melocactus. T.

Glaucium $\mathrm{T}$. corolla rosacea -. - - Chelidonium. 
Polygonatum T. corollâ tubulosâ - - - Lil. convallium. Centaurium minus T. cor. infundibulif. Gentiana. Liliastrum T. corolla hexapetala - - Hemerocallis. Borbonia P. corollâ fentaphylioideâ - - Laurus. Benzoë B. corollâ octofidâ - - - - Laurus. Auricula ursi T.corollâ hypocrateriformi. Primula. Triphylloides T. corollâ monopetalâ - Trifolium. Oxycoccus T. corollâ tetrapetalâ - - Vaccinium. Bonarota M. corollâ tubulosâ - - - Veronica. Zannonia P. corollâ tripcta!âa - - - Commelina. Borraginoides B. corolla infundibulif. Borrago. HorminumT.c. galeâ galeatâ, barbâ conc. Salvia Sclarea T.c. galeâ falcutâ, barlấ concavâ Salvia. Phelypxa P. corolle galeâ bifidâ - - Clandestina T. Murucuja T. Neciario indiviso - - Passiflora. Sherardia V. stamin. 2 _. . - . - Verbena. Stellaris D. stamin. non planis - - Ornithogalum. Porrum T. stamin. trifidis - - . - - Allium. Dodonæa P. fiore 3 fido - - - - Ilex.

Hypocistis T. flore quadrifido - - Asarum. Radiola D. fiore quadrifido - - - - Linum. Unifclium D. flore quadrifido - - - Convallaria. Bernhardia A. fioribus dioicis - - - Croton. Petasites T. floribus fasciculatis - - Tussilago. Ananthocyclus V. floribus flosculosis - Cotula. Ceratocephalus V. floribus radiatis - Bidens. Doria D. flosculis radii raris - - Solidago.

Mitto genera Kuppiana non definita, Knautiana, et aliorum, quibus suffecerit bene velle, e. gr.

Medium Campanulæ fr. sloculari.

Speculum Veneris Campanula fr. siliquoso.

Cornucopioides Valerianæ fl. irregulari.

Limonioides Statices $f$. monopetalo.

Viscaria Silenes fr. sloculari.

Tetragonolobus Loti fr. angulari.

Ergo, nisi canon assumeretur, tot genera evaderent, quot species existunt.

I 7 . In plerisque Generibus nota aliqua Fructificationis singularis (105) observatur.

Denticuli Staminum in Brunellâ, Toreniâ, Euphrasiâ, Alysso, Crambe. 
Stamen mutilatum: Curcuma, Cbelone, Bignania, Martynia. Pori nectariferi in unguibus petalorum Ranunculi.

Rime clause intra corollam Hydropbylli.

Nectaria tubulosa, Hellebori, Nigelle.

Hyoscyzmus capsulà operculatâ distinguitur a Physalide. Pancratii stamina nectario inserta, diversa a Narcisso. Resedie nectarium laterale, corollâ, et pistillo vario. Cainpanule nectarium 5 valve, capsula, et corolla varia. Iris stigna singulare, corollæ barba varia.

172. Si nota aliqua fructificationis singularis (105), vel sui generis propria $(171)$ in speciebus non omnibus adsit; ne plura genera accumulentur, - cavendum.

Erica, et Andromeda antea genere juncta fuere, sed anthere bicornes Erica proprixe.

Ranunculus adsumsit antea Adonidem, sed Adonis caret poris nectariferis.

Aloz, et Agave idem genus constituebant, sed stamina in hac non receptaculo, at corollæ inserta, utrumque genus distinguunt.

I73. Si nota generi singularis (105) etiam in genere affini reperiatur, ne idem genus in plura, quàm natura dictitat, separetur, cavendum.* Sedum, Sempervivum, Rbodiola, Crassula, Tillea, Cotyledon nectariis pistilli basi adhærentibus.

- Epilobium, Oenotbera calyce tubuloso. MIespilus, Crategus, Sorbus floris structurâ. Alnus, Betula flosculis tribus supra foliolum amenti.

174. Quo constantior pars aliqua fructificationis ( 167 ) est in pluribus speciebus, eo etiam certiorem exhibet notam genericam. *

$H_{y p e c o i}$ Nectarium, non Siliqua constans est.

Convallarie Bacca maculata, non Corollâ Lilii convallii, $\mathrm{Po}$ lygonati, Unifolii.

Cassize corolla, non siliqua.

Lobelice corolla, non fructus Lobelie, Cardinalis, Rapuntii, Laurentice.

Verbence calyx, et corolla, non stamina, et semina Sherardice $V$. 
I75. In aliis Generibus hæc, in aliis alia pars fructificationis constantior observatur, nulla verò constantissima est. *

Pericarpium diversum in congeneribus docet Impatiens, Campanula, Primula, Papaver, Cistus, Fumaria, Arbutus.

Calycem: Nymphea, Cornus.

Corollam:Vaccinia, Convallaria, Andromeda, Gentiana, Linum.

Stamina: Corispermum, Valeriana.

Semina: Ranunculus, Alisma.

1 76. Si Flores $(87)$ conveniunt, Fructus (87) autem differunt; ceteris paribus, conjungenda sunt Genera.

Flores similes, fructus diversos in eodem genere existere docent Cassia, Hedysarum, Sophora, Lavatera, Hibiscus, Mimosa.

I77. Fizura (95) Floris $(87)$ certior est, quèm Fructûs (87); Proportio (95) partium autem maximè diversa, nec constantissima.

Florem certiorem esse fructu, patet plurimis exemplis:

Campanula, Medium, Speculum veneris.

Primula, Auricula ursi.

Antirrhinum, Elatine, Asarina.

Alismia, Dimasonium.

Hibiscus, Malvaviscus.

Cistus, Helianthemum.

Fumaria, Corydalis.

Arbutus, Uva ursi.

Clematis, Viticella.

Guilandina, Zygophyllum; Papazer.

Ranunculus, Hesperis, Datura.

178. Numerus (94) faciliùs aberrat, quàm Figura (35), proportione numeri tamen optimè explicatur; Flores autem numero in eadem planta diversi secuudum primarium considerandi. *

Flores numero diversi in eadem planta.

Ruta flos 5 , flores 4 , confirmat Pseudo Ruia Mich.

Chrysosplenii $5,-4$, Saxifraga.

Monotrope $5,-4, \longrightarrow$ uniflora. 


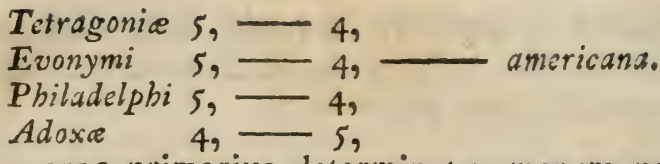

ergo primarius determinat numerum naturalem. Affinitates numeri fructificationis propriæ.

Floris X. et VIII. Stamina in eadem : Rute, Monotropa.

in diversis : Vaccinii, et Oxycocci $T$.

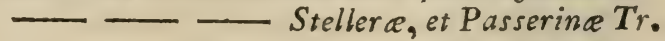

- Sedi, et Rhodiola $L$.

Y. - Arenarice, et Morbingice.

V. et IV. Corolla in eadem : Evonymus, Ruta, in diversâ : Linum, et Radiola D. - Anagallis, et Centunculus.

Calyx in diversâ: Nymphea lutea, et alba.

Stamina in eadem : Evonymus. in diversâ : Mimosa......

IV. et III. Flos in eadem : Asperula Rubeola $T$. in diversâ: Paris europ. et americ. Ilex, et Dodona Plum.

Eructûs V. et III. frequentissimè proportionati.

Rute cum Pegano.
Evonymi- Celastro.
Nigelle - Garidellâ.
Cisti T. - Heliantbemo T.
Aconiti - Anthorâ.
Viscarice - Silene.

V. et IV. Rute, Evonymi, Philadelphi, Lini, et Rbodiolse.

r79. Situs (97) partium constantissimus est. Receptaculi (86) situm in Ordinibus (16I) magni fecit Tournefortius (64).

TOURNEFORTIUS calycem abire in fructum, rectiùs intellexit, quàm verè dixit; idem enim est, ac germen vel supra elevatum, vel infra perianthium detrusum, ut in Saxifragâ, et Geo T.

Situs partium rarissime in congeneribus mutatur. 
Ambit internè calycis parietes receptaculum in Icosandris, aliisque, unde corolla, $\in t$ filamenta calyci inseruntur in Fragariâ, Pyro, Oenotherâ.

180. Petalorum $R$ egularitatern nimiam fecit Rivinus (6I).

Umbellate alice gaudent corollis regularibus, alice irregularibus.

Gerania Europeca corollâ regulari, Africana irregulari.

$\boldsymbol{L} y c o p$ sis, et Echium, inter regulares Asperifolias, irregulari corollà gaudent.

18ı. Nectarium (I Io) Maximi fecit Natura.

Nectarium ne nomine notum erat, antequam idem determinavimus.

Orchis, Satyrium.

Monotropa, Fumaria, Viola. Nigella.

Malpighia, Bannisteria. Aconitum.

Adenanthera, Commelina. Parnassia.

Laurus, Helsine.

Dictammus.

Zygophyllum.

Szvertia.

Lilium, Fritillaria.

Hydropbylluin.

Ranunculus.

Epimedium.

Theobroma.

Reseda.

Grezvia.

Helleborus, Isopyrum.

Tropeolum, Impatiens.

Hermannia.

Eerberis.

Stapbylea.

Passiflora.

Narcissus, Pancratium.

Rirabilis.

Nerium.

Stapelia, Asclepias.

Diosma.

C.mpanula, Plumbago.

Hyacinthus.

$R$ hododendrum.

Cheiranthus, Sinapis.

Kiggelaria, Clutia. 
182. Stamina, et Calyx, luxuriationibus minừs obnoxia, petalis longè certiora sunt.

Corollæx figurâ diversæ in eodem genere multæ sunt: Vaccinix, Pyrole, Andromeda, Nicotiane, Mlenyanthes, Primule, Veronica, Gentiance, Hyacinthi, Scabiose, Narcissi。* - numero diversæ in distinctâ specie: Ranunculus Pentapstalus, Polypetalus, * Helleborus Pentapetalus, Polypetalus. Statice Pentapetala, Monopetala. Fumaria Dipetala, Tetraperala.

- Carica, Jatropha.* $*$ in specie.

183. Pericarpii structura $(92)$ ab antecessoribus Botanicis trita, innumeris exemplis docuit se minùs valere, quàm ii crediderant.

Genera ficta fundamento fructûs superstructa, nec habito respectu sanæ theoriz, plurima introducta sunt: ${ }^{*}$ e. gr. Clandestina T. Fructu elastico non esset Anblatum T. Trollius R. Fructu multicapsulari . Helleborus. Sesamoides T. Fructu multicapsulari .. Reseda. Lycopersicon T. Fructu multiloculari . Solanum. Ascyrum T. Fructu qinqueloculari . Hypericum. Dortmanna R. Fructu biloculari .... Rapuntium T. Helianthemum T. Fructu uniloculari . Cistus. Androsæmum T. Fructu uniloculari . Hypericum. Pavia B. Fructu uniloculari ...... Esculus. Asarina T. Fructu multivalvi .... Antirrhinum. Elatine D. Fructu latere debiscente . Antirrhinum. Nelumbo T. Fructu apice perforato. Nymphæa. Raphanistrum T. Fructu articulato . Raphanus. Cakile T. Fructu articulato...... Bunias. Ulmaria T. Fructu contorto ...... Filipendula. Persica T. Fructu succulento ..... Amygdalus. Cassia T. Fructu succulento ..... Senna T. Inga P. Fructu succulento ....... Acacia $\mathrm{T}$. Malvaviscus D. Fructu succulento . . Hibiscus. Lobelia P. Fructu drupaceo ..... Rapuntium T. Pereskia P. Fructu folioso . . . . . Cactus. Sabina B. Fructu verrucoso ...... Juniperus. Bihai P. Fructu trispermo . . . . . Musa. 


\section{H A R A T ERES.}

Alaternus T. Fructu trispermo ..... Rhamnus.

Frangula T. Fructu dispermo ..... Rhamnus.

Dracunculoides B. Fructu monospermo Hemanthus.

Onobrychis T. Fructu monospermo . . Hedysarum.

Malvinda D. Fructu non inflato ... Abutilon T.

Cysticapnos B. Fructu inflato .... Fumaria.

Impatiens R. Fructu attenuato .... Balsamina T.

Guazuma P. Fructu reticulato .... Cacao T.

Paliurus T. Fructu clypeiformi .... Rhamnus.

Alisma D. Fructu corniculato ... Damasonium T.

Securidaca T. Fructu gladiato .... Coronilla.

Melo T. Fructu ovato ....... Cucumis.

Nelopepo T. Fructu sulcato ..... Cucurbita.

Rapistrum T. Fructu non debiscente. Crambe.

Radicula T. Fructu siliculoso .... Sisymbrium.

Blattaria T. Fructu rotundiore .... Verbascum.

Persea P. Fructu undique baccato .. Laurus.

Cururi P. Fructu ad apicem seminifero. Seriana P.

Bursa pastoris T. Fructu non marginato . Thlaspi.

Nasturtium T. Fructu marginato .. . Lepidium.

Valerianella T. Fructu non papposo. Valeriana.

Anemonoides D. Seminibus nudis... Anemone.

Eupatoriophalacrum V.Seminibus nudis. Verbesina.

Leontodontoides T. Seminibus subnudis. Hyoseris.

Atractylis V.Seminibus coronâ obsoletâ . Carthamus.

Carthamoides V. Seminibus papposis. Carthamus.

Zazintha T. Seminibus papposis ... Lapsana.

Alypum N. Seminibus papposis ... Globularia.

Xeranthemoides V. Pappo plumoso ... Xeranthemum.

Asteropterus V. Pappo plumoso .... Aster.

Acarna V. Pappo plumoso ....... Cnicus.

Achyrophorus V. Pappo plumoso .. Hypochæris.

Carlinoides V. Pappo obsoleto .... Carlina.

Viticella D. Seminibus caudatis ... Clematis.

Nymphoides T. Seminibus arillosis. Menyanthes.

Karatas P. Seminibus non arillatis . Bromelia.

Tragopogonoides V. Seminibus incurvis Tragopogon,

Tinus T. Seminibus pyriformibus ... Viburnum.

Opulus T. Seminilus cordatis .... Viburnum.

Persicaria T. Seminibus triangularibus Polygonum.

Emerus T. Seminibus cylindricis ... Coronilla.

Foniculum T. Seminibus crassis ... Anethum. 
Lens T. Seminibus lentiformibus ... Cicer.

Pepo T. Seminibus non emarginatis .. Cucurbita.

Falearia D. Seminibus gracilibus ... Sium.

Cerinthoides B. Seminibus 4. distinctis . Cerinthe.

Blieria H. Seminibus echinatis ..... Sherardia V.

I84. Luxuriantes flores (Ir9), Eunuchi ( 150 ), et Mutilati ( I 9), ut Monstra, calculos in generum constitutione non ferunt.

Pieni flores si characterem intrarent, plerisque plantis nullus numerus petalorum adsignari posset, et in plerisque characteribus Stamina excluderentur.

Mutilati( I I )excludunt corollam, adeoque etiam in charactere e. gr. Campanulve, Ipomøe, Ruellice, \&c. \$. I I 9. corolla excluderetur, contra naturam reliquarum specierum.

I85. Multiplicati ( 120$)$, et Pleni ( $12 \mathrm{r}$ ) flores a Perianthio, et infimâ serie petalorum, uti Proliferi (122) a prole, judicantur.

Flos plenus ad Genera referri nequit, cùm monstrosus sit; fiet hic naturalis, si in solo macro seratur.

Perianblium in flore pleno non mutatur, hinc ad genus sæpius refertur flos plenus secundùm Methodos Calycinas; e. gr. Hepatica, Ranunculus, Alcea.

Corollee Polypetale infima Series numero constans permanet

etiam in plenis; hinc numerus petalorum inde sæpius facillimè eruitur, ut in Papavere, Nigellâ, Rosâ.

186. CHARACTER est Definitio Generis ; isque triplex datur: Factitius (188), Essertialis (187), et Naturalis (189).

Character genericus idem est, ac Definitio generis. HABITUALIS ab Habitu $\left(\oint_{\circ} 16_{3}\right)$, quem adsumsere veteres, post detectam fructificationem $\left(\mathrm{I} 6_{4}\right)$ hodie per se exolevit in generibus.

r87. ESSENTIALIS Character (186) notam Generi, cui applicatur, propriissimam ( 171 ), et singularem (105) subministrat.

ESSENTIALIS Character unicâ ideâ distinguit Genus à congeneribus sub.eodem Ordine naturali. 
Exempla Syst. Natur. editio 6. ${ }^{2}$ plures habet, quod vide: Nyctanthes, Circea, Gratiola, Salvia; Olax, Iris, Melica; Leucadendron.

Flantago, Epimedium; Hydrophyllum, Mirabilis, Hyoscyamus, Physalis.

Stapelia, Ceropegia, Etthusa, Parnassia, Statice.

Galanthus, Narcissus, Pancratium, Asphodelus, Aloë, Hemantbus.

Tropeolum; Laurus; Anacardium, Dictamnus, Zygopbyllum. Melia, Diantbus, Phylolacca; Bixa; Reseda, Delpbinium. Aconitum, Nigella, Liriodendrum, Uvularia, Ranunculus, Helleborus.

Hyssopus, Brunella, Scutellaria, Euphrasia, Torenia, Latbrea, Craniolaria.

Halleria, Acanthus, Cranibe, Alyssum: Hermannia, Pentapetes.

Hibiscus; Polygala, Amorpha, Dalea, Psoralea; Theobroma; Echinops, Inula, Centaurea, Corymbium, Jasione, Impatiens; Sisyrinchium, Passiflora, Aristolocbia, Helicteres, Arum, Zostera; Zizania, Liquidambar, Clutia.

Character essentialis quò brevior, eò etiam præstantior est. Facillima plantarum dignotio per characteres essentiales acquiritur.

188. FACTITIUS Character (186) Genus ab aliis Generibus, ejusdem tantùm ordinis artificialis, distinguit.

Factitius Character distinguit genera extra ordinem naturalem, sive pauciores, sive plures notas characteristicas assumit.

DiagnosisCharacterisNaturalis esthæc, quod nunquam valeat in ordine naturali genera distinguere; Characteres itaque, sive essentiales, sive naturales, quæ nequeunt genera in ordine naturali sufficienter distinguere, factitii dicendi sunt. Rajus Characterem factitium detinit, statuendo Notas generum characteristicas non sine necessitate multiplicandas esse, nec plüres, quaim quibus opus est, ad genus certo determinandum coacervandas.

189. NATURALIS Character ( 186 ) notas omnes (92-I I 3) genericas possibiles (167) allegat; adeoque Essentialem (187),et Factitium(188) includit.

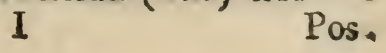


Possibiles omnes notas addicit character naturalis, exceptis tamen is, quas structura naturalissima (93-97) subministrat.

Ego primus hos characteres composui.

Includit in se omnes characteres possibiles;inservit omni systemati; $D$ asim sternit novis systematibus; immutatus persistit, licèt infinita genera nova detegerentur.

Corrigitur novis detectis Speciebùs tantummodo, exclusione scilicet notarum superiuarum. Gen.pl. pref. 20.

Prarogativa, et Usus Characteris naturalis colligitur ex Gen. pl. pref. I8.

390. Character Factitius (188) succedaneus est; $E$ ssentialis ( 187 ) optimus, sed vix ubique possibilis; Naturalis, difficillimè elaboratur ; elaboratus autem Basis ( $15^{6}$ ) est omnium systematum (53), Generum (I 59) infallibilis Custos, omnique Systemati (26-37) possibili, et vero applicabilis.

F ACTITIOS Characteres proposuerunt illi, qui in methodo artificiali ( 162 ) notas adduxere, quibus genera, sub eodem ordine disposita, distingui potuere; sic Rajus, Tournefortius, Rivinus, et ante nos plerique.

ESSENTIALES Characteres sunt ejusmodi ; qui in ordine naturali valent distinguere genera maximè affinia unâ, alterâve notâ; adeoque nequeunt non ejusmodi valere, etiam ubi genera affinia segregantur; quales plurimos adduxi in Syst. Nat. edit. $60^{a}$

NATUR ALIS Character tradit omnes notas Characteristicas possibiles, adeoque omnibus methodis inservit, quamvis innumerx in dies detegerentur.

Genera mea plantarum promunt Characteres naturales, adeoque inserviunt omnium methodis, et fundamentum præbent et antiquis, et novis.

391. Naturalis Cbaracter (189) ab omni Botanico (7) teneatur, oportet.

Si oninium generum characteres Essentiales detecti essent; facillima evaderet plantarum cognitio, et multi suo damno Characteres Naturales flocci penderent:at sciant nullum, sine notitiâ characteristicâ natụrali, evasurum solidum 
Botanicum; novis enim detectis generibus, destitutus Charactere naturali Botanicus semper vacillabit. Qui credit se sapere in Botanicis ex Essentiali Charactere, neglecto Naturali, is itique fallit, et fallitur; cùm essentialis, novis detectis generibus, nequit non sæpius fallax evadere.

Character naturalis generum plantarum fundamentum est, quo destitutus nullus de genere rite judicavit; adeoque absolutum fundamentum cognitionis plantarum est, et erit.

192. Character Naturalis (189) fructificationis notas omnes differentes (98), et singulares ( $\mathrm{I}_{05}$ ), per singulas suas species ( 157 ) convenientes ( 165 ), recensebit; dissentientes (166) verò sileat.

Est opus infiniti laboris, antequam Characteres secundùm omnes species fuerint limitati.

Omnes partes fructificationis examinandæ, etiam quæ visum eludunt, quamvis Microscopium adhiberetur (quod tamen rarissimè adhibere necesse est), quum, ignolâ fructificatione, nulla de genere certitudo: qui velit Acarum farinæ, vel scabiei nosse, debet utique uti microscopiis. Rajus itaque (perperam): Note obvic sint, manifeste, et cuilibet facilè observabiles: Nam cùm Methodi usus precipuus sit rudes, et Tyrones in Stirpium cognitionem compendi absque tedio, et difficultate inducere, non oportet ejusmodi notas proponere, quce adtentum, et solicitum requirunt spectatorem, cuique, ut microscopium secum ferat, necessum sit.

193. Nullus character infallibilis est, antequam secundùm omnes suas ( I 39) species ( I 57) directus est. Botanicus consummatissimus, isque solus, optimum conficit Naturalem Characterem ; fiet enim consensu specierum plurimarum; omnis enim species excludit notam aliquam superfluam.

Fit character naturalis accuratissimâ descriptione fructificationis prima speciei; omnes reliquæ species generis conferantur cum primx, ut excludendo notas dissentientes omnes, tandem elaboratus evadat.

194. Inflorescentia ( $\left.16_{3}\right)$ notam Characteristicam non dabit.

Locus, quem occupat in Herbá Fructificatio, characteri- 
stica nota non est, quamvis aliter visum Rajo, Rivino, Heuchero, Knautio, Kramero, aliisque, sed pestis Botanices, adeoque ut damnosissima repudiata a summis Botanicis Tournefortio, Vaillantio, aliisque.

Modos vide ad Pedunculum $\$$. 82 . et 163 .

Rajus, Boerbave, Riviniani, aliique huic locum admittebant in charactere, ut eo arctiùs naturæ adhærerent, quam amisere citiùs.

195. Character nomen Genericum in frontispicio gerat.

Fohrerius in Hodego ex Charactere Nomen concludit: e. gr. si flos hujusmodi, erit hoc nomen. Non enim argumentamur a Craractere ad Nomen, sed judicamus Genus secundùm nomen Genericum, cujus essentia comprebenditur Charactere.

196. Unaquæquc Species Fructificationis (86) in Charactere naturali ( 189 ) novam ordiatur lineam. Distinctè ut omnia tradantur, hoc fiet; inde mox partem quæstionis reperio; inde si quid deficiat, mox observo.

197. Nomen partis fructificantis (86) lineam (196) differentibus literss inchoabit.

Differentes literæ, ut faciliùs perspiciatur character, et evidentior evadat; contra autem factum ab antecessoribus omnibus.

198. Similitudinis notam ( 167$)$, nisi dextrâ manù notiorem, Character (192) nullus assumat.

Omnis similitudo claudicat.

Similitudo a nobismet ipsis semper præsens est; $a b$ animalibus non omnibus constat; $a b$ artificialibus admodum mutatur.

Volsella T. Perianthio Urticx - - Chirurgo. Mitra polonica T. in Corollâ Aconiti - Hifloricis. Mitra episcopalis T. in Capsulâ Mitellæ - Theologis. Ossa byoidea $\mathrm{T}$. in Filamentis Salviæ - Anatomicis Caput vipere T. in Seminibus Anchusæ - Zoologis.

199. Notas convenientes (192) terminis compendios describat character. 
Terminos artis nosse itaque primùm $\mathrm{T} y$ ronis erit.

Ger. plant. pref. 25. Explodendi sunt pomposi, et eloquentiæe flosculi.

Oratorio stylo in Charactere nil magis abominabile.

Termini artis faciunt, ut paucis ideas nostras exponamus. LINI character oratorius sit exempli loco:

Extimum floris tegumentum viride, quod ante explicatio. nem includit forem, est ad basim in 5 partes æquales veluti sectum, ita tamen ut unaquxque pars sit longior, quàm lata, et versùs utramque extremitatem angustata, desinentibus summis apicibus in acumen; ceterum hæ 5 partes perpendicularem situm servant, et ratione foliorum floris admodum breves sunt : nec diffluat cum florum foliis coloratis, sed permanent ad fructûs maturitatem. Intra hæc folia sunt alia Folia etiam numero quinqui, sed tenera, colorata, etiam oblonga, sed sursum magis magisque dilatata, ferè uti infundibulum mechanicorum; sunt etiam hæc longè majoia, quàm exteriora viridia folia. Tum intra hæc quinque colorata magna folia floris sequuntur numeros partes filiformes, supernè sensim attenuatæ in mucronem, qux perpendiculares ferè sunt, nec longitudine excedunt extima foliola floris; harum summitatibus insident totidem corpuscula, simplicia, crassiora, quß spargunt farinam, et basi fissa in 2 partes acutas. Hisce paribus probe perspectis in centro floris occurrit corpus, quod excrescit in fructum, et sub florescentiâ ferè globi formam imitatur, cui superimposita sunt 5 fila vegetabilia, quæ ubique eamdem servant crassitiem, et perpendicularem ferme situm habent, eamdenque obtinent longitudinem cum 5 partibus filiformibus mox descriptis; sunt autem hæc apicibus non capitata, aut incrassata, sed parùm extorsum flexa; peractâ forescentiâ fit Fructus aridus, formâ ferme globi, sed obsoletè 5 angulis notatus, et in summitate gerit mucronem; hunc fructum si transversim disseces, videbis eumdem internè in Io concamerationes divisum, et cùm sponte dehiscat, se aperire in 5 partes æquales : intra quas ro latuere semina figurâ ferme ovi, sed magis langa, et alterâ extremitate acuminata, nec non parùm compressa, superficie quasi politâ, et glabrâ.

Character idem Lini, linguâ Botanicis usitatâ, eadem omnia complectens. 
CAL. Perianthium sphyllum; foliolis erectis, lanceolatis, acutis, parvis, persistentibus.

COR. Infundibuliformis, spetala: Petalis cuneiformibus, obtusis, patulis, magnis.

STAM. Filamenta 5. subulata, erecta, logitudine calycis. Filamenta 5 alia, alterna, emarcida. Anthere sa gittatæ.

PIST. Germen ovatum. Styli s, erecti; filiformes, longitudine staminum. Stigmata simplicia, reflexa.

PER.Capsula subglobosa, subpentagona, 5 ralvis, I olocularis. SEM. Solitaria, ovata, planiuscula, acuminata, glacerrima. 200. TERMINI puri ( 8 I -85 ) eligendi; obscuri, et erronei non admittendi sunt.

Ger. plant. pref. 26. Dubia potiùs omittenda, quàm dubiè defendenda.

Rajus meih. Notæ generum tam summorum, quàm subalternorum claræ sint, distinctæ, et exactè definitær, non obscuræ, et indeterminatæ. quarum significatio,

Mihi. quousque extendatur, incertum est.

MASCULUS Fl.Sterilis Tourn.Paleaceus Raj.Abortiensaliis. APETALUS Tourñ. Imperfectus Riv. Knaut. Pont. Stamineus Raj. Incompletus Vaill.

PETALODES. Tourn. Perfectus Raj. Riv. Kram. Pont. CALYCULATUS. Completus Vaill.

IR RE GU LARIS Riv. Difformis Jung. Kn. Anomalus'Tourn. RINGENS.Labiatus Tourn. BarbatusRiv. Personatus Tourn. MULTIFIDUS, Laciniatus Tourn. Monopetaloides, \&c. aliis. COMPOSITUS Tourn. Riv. Conglobatus Pont. Aggregatus Kn. Capitatus Raj.

PLANIPETALUS Raj. Semiflosculosus, Tourn. Lingulatus Pont. Cichoraccus Vaill.

RADIATUS Tourcef, Stellatus Morison.

DISCCS Tournef. Umbo Moris.

ANTHER A.ApexRaj.Tourn.Riv.Capsula staminis Malpig. RECEPTACLLUM Pont. Sedes Raj. Placenta Boerh. Thalamus Vaill.

AMEI TLM Tourn. Julus, N'ucamentum, Catulus aliis.

STROBILUS. Conus aliis.

DRUPA kyber. lex. 1 50. Prunus aliis, Fructus mollis ossiculo Tournef.

GYMNOSPERMUS fructus, Herm. Semina nuda Riv. AN- 
ANGIOSPERMUS fructus, Herm. Semina Pericarpio tecta Riv.

CLASSIS. Ordo Tournef. Genus summum Raj. Riv. ORDO. Sectio Tourn. Genus subalternum Raj. Riv.

20I. Termini (199) necessariis (200) plures excludendi, pauciores augendi sunt.

Auxi Botanicem plurimis terminis $\$$. 82.83 .84 .85 .86 . e.gr. Involucrum, Spatha, Corolla, Anthera, Pollen, Germen, Stigma, Legumen, Drupa, Cyma, Arillus, Stipula, Scapus, Bractea, Pedunculus, Giandula.

Termini præservarunt Anatomian, Mathesin, Chemiam ab id:otis; Medicinam autem eorum defectus conculcavit. Usui eorum eximius est in compendiosè loquendo, et rite co. gitando, modò non destituantur adæquatis definitionibus. Dissepimentum valvis contrarium, aut parallelum in Siliquosis sæpius adhibuit Tournefortius, quod intelligendum cum grano salis; parallelum dicitur, cum latitudine, et diametro transversali accedat ad valvulas; contrarium verò audit, ubi angustius est dissepimentum, quàm valvulæ

Papilionacea corolla ab insecto dicta, concipitur vulgò ut navicula, cujus

Carina excipit, et includit stamina, et pistilla, constatque duobus petalis coadunatis.

Ale autem carinæ lateribus utrinque adstant solitariæ. $V$ exillum alis, et carinæ incumbit.

Ringens corolla monopetala irregularis est, et limbo divisa in duo lábia.

Galea Riv. labium superius est.

Barba verò labium inferius audit.

202. Character (192) in omnibus, licèt diversissimis Systematibus (54-77), immutabilis servetur.

Quamdiu summi Systematici novos introducebant characteres, novosque conceptus generis, tamdiu barbariei exposita gemuit Botanices scientia tempore Raji, Tournefortii, Rivini, Boerhaavii, Knautii, aliorum.

Nunc verò pacatis magis rebus, quamquam novæ methodi introductæ, nulla inde calamitas Botanices; ut patet ex scriptis Gronovii, Royeni, Wachendorffii, Gmelini, Guettardi, Dalibardi \&c. 
203. Genus ( I 59) unicâ specie ( I 57) constare potest, licèt plurimis sæpius componatur.

Unicâ specie constant plurima genera: sic

\begin{tabular}{|c|c|c|c|}
\hline $\begin{array}{l}\text { Parnassia, } \\
\text { Tamarindus, } \\
\text { Lagecia, } \\
\text { Pentborum, } \\
\text { Heliocarpus, } \\
\text { Orvala, } \\
\text { Obolaria, } \\
\text { Dalea, } \\
\text { Hura, }\end{array}$ & $\begin{array}{l}\text { Epimedium, } \\
\text { Cormucopie, } \\
\text { Gloriosa, } \\
\text { Neurada, } \\
\text { Calligonum, } \\
\text { Halleria, } \\
\text { Liniosella, } \\
\text { Corymbium, } \\
\text { Valisneria, }\end{array}$ & $\begin{array}{l}\text { Hydrophyllum, } \\
\text { Diapensia, } \\
\text { Petiveria, } \\
\text { Garcinia, } \\
\text { Hepatica, } \\
\text { Dodartia, } \\
\text { Anastatica, } \\
\text { Nepzntbes, } \\
\text { Humulus. }\end{array}$ & $\begin{array}{l}\text { Butomus, } \\
\text { Coris, } \\
\text { Anacardium, } \\
\text { Menizelia, } \\
\text { Trichostema, } \\
\text { Craniolaria. } \\
\text { Anorpbu, } \\
\text { Cynomorium, } \\
\text { Arctopus. }\end{array}$ \\
\hline
\end{tabular}

Plurimis speciebus constant alia Genera, Botanicis in specie commendanda.
Mesembryiznth. Euphorbia,
Sedum, Geranium,
Allium,
Alnë,
Convolvulus,
Campanula,
Erica,
Saxifraga,
Silene,
Solanum,
Statice,
Mimosa,
Cassia,
Prentilia,
Gentiana,
Antiríbinum,
Polygala,
Aster,
Carex,
Hypericum, Hibiscus,
Ranunculus,
Chenopodium,
Phaseslus, Astragalus, Hedysarum, Gnaphalium, Centuurea, Salix, Ficus,
Buphthalmum.

204. Quod valet de charactere generico, valet etiam de classico (160), licèt in hoc latiùs'sumantur omnia.

Generum genus est Ordo, ordinum autem genus Classis est. Valent itaque hîc canones dati S. 164 ad 202.

205: Classis ( 160 ) genere ( 159 ) magis arbitraria ( 162 ) est, utrisque magis Ordo (i6r).

$\mathrm{Classis}$ vocabulum in denominatione minùs nocet, si aberret, quàm Generis nomen.

Cave, ne seducaris ab afinitate Generum cum Classibus, et Ordinibus, ne ordines naturales ad Genera reducas, - inmo tandem et Classes. 1

a. Malva, Altha, Alcea, Lavatera, Urena, Hibiscus. ord. 34. b. Sedum, Sempervivum, Cotyledon, Crassula, Tillea.ord. 46. c. Cactus, Mesembry:anthemum, Aizoon, Tetragonia. ord. 46. d. Lycbnis, Coronaria, Agrostema, Silene, Diantbus, Saponaria, Cerastium, Spergula, Arenaria, Morbingia, Sagina. ord. 42 .

Sic 
Sic ex Ordinibus Columniferis, Stellatis, Umbellatis, Siliquosis, Verticillatis fierent totidem genera, sed adeo vasta, ut barbariem inducerent.

Orcbidece (ord.4.) omnes in idem Genus confluerent, et Scitanina (ord. 3.) in alterum, et hi demum in unum; sic primum Casus Botanices, et ruina ex proprio generum pondere.

206. Classes, quò magis naturales, eò, ceteris paribus, præstantiores sunt.

Aftines conveniunt habitu, nascendi modo, proprietatibus, viribus, usu.

Summorum Botanicorum hodiernus labor in his sudat, et desudare decet.

Methodus naturalis hinc ultimus finis Botanices est, et erit. ObicemMethodi naturalis objecere tria præprim is obstacula. a. Neglectus Habitus plantarum, post excultam doctrinam fructificationis, præsertim foliationis novæ p. 105.

b. Defectus Generum Exterorum, nondum detectorum.

c. Affinitas Generum áb utraque parte.

Linnea media est inter Stellatas 44, et Valerianam 18 , et Loniceras 63 .

Cornus connectit Stellatas 44, Aggregatas 18, Dumosas I 9.

Juncus Calamariasi 3, Gramina r 4, Coronarias 9, sociat. Dodecatbeon Cortusam, et Cyclamen conciliat. Hyoscyamus calyce inflats $\mathrm{H}$. ups. 2. Physalidi affinis fit. Hibiscus petiolis floriferis H. ups. I. Turnere itidem petiolis floriferis adsociatur; sine cognitione Petioli fructificantis nemo indagaret Turneræ affinitatem cum Hibisco.

207. Classes, et Ordines nimis longæ, vel plures difficillimæ sunt.

Sexuali Methodo Classis Pentandrie, et Syngenesire vastissimx, adeoque in hoc Systemate, præe reliquis difficiliùs, in his distinguuntur Genera.

Ordo Pentandrice Monogynice ob copiam Generum difficiliùs distinguitur; ut aliorum Methodos intactas relinquam.

Boerbuave classes 33 habet; Knautius 8 ; ideoque prioris ordines erunt plures, et breviores; posterioris autem pauciores, at vastissimi. 
208. Ordo Genera inter se magis affinia proxime collocabit.

Raj. Meth. 5. Providendum est, ne plantæ cognatæ separentur; dissimiles, et alienæ cunsocientur.

Quæ difficiliùs distinguuntur, propriùs collocentur.

Exempli loco sit Tetrandria monogynia, ubi Ordines $\mathrm{Na}-$ turales Stellate 44. Calycanthema 40. Aggregate I 8 ; hi naturales Ordines; si possibile, disctincte in ordine eodem tradendi, nec miscendi, quamquam aliter fieret facilior divisio.

rectè. 18. Leucadendron.

Protea

Cephalanthus.

Globularia

Dipsacus

Scabiosa

Knnutia

44. Asperula

Sherardia

Spermacoce

Hedyotis

Knoxia

Diodia

Crucianella

40. Luduvigia

Oldenlandia

Isnardia

Ammannia. malè. Leucadendron 18. Asperula 44. Ludwigia 40. Protea I 8.

Sherardia 44. Oldenlandia 40. Cephalanthus 18. Spermacoce 44. Isnardia 40. Globularia 18. Hedyot is 44. Ammannia 40. Dipsacus 18. Knoxia 44. Scabiosa I 8. Diodia 44. Knautia I 8. Crucianella 44.

Tournefortiam in Pentandria Monogynia ob Baccas lubenter separassem ab Asperifoliis, nisi repugnasset natura. Quæ sub eodem ordine militant, non debent separari interjectis aliis generibus, quando ita affines sunt, ut vix limites admittant; Sic
Alsine,
et Arenaria
Primula,
et Androsace
Lysimachia,
et Anagallis
Lonicera,
et Diervilla
Convolvulus, et Ipomeca
Chenopodium, et Beta
Solanum,
et Capsicum. 


\section{CHARACTER E S.}

209. Habitui plantarum $\left(16_{3}\right)$ adeo adhærere, ut rite adsumta Fructificationis principia (164) deponantur, est stultitiam sapientiæ loco quærere.

Inflorescentia, seu Situs Fructificationis in axillà, racemo, corymbo (unde characteres habituales $\$$. 168) causavit infinita Genera falsa, præsertim Knautio, Kramero, similibusque.

Facies plantarum, Veterum lapis lydius, Recentiorum coticula, magni ab omni Botanico facienda est, sed et his modus adsit.

Bidens (a) folio non dissecto. Tourn. Fl. suec. 664. gerit fructificationem exactè generis Bidentis, at BIDENS (b) folio non dissecto, flore aureis petalis undique radiato. Moris. gerit fructificationem absolutè Coreopsidis; cùm $a$ careat radio, $b$ verò instruatur radio ex petalis 8 sterilibus, erit prior $a$ Bidentis, $b$ verò Coreopsicis generis. Sed dubium adhue maret, numne planta radiata $b$ sit varietas non radiatæ $a$; quod si esset, uti verisimile videtur, non licet has duas plantas separare; nec consultum ducerem genera Bidentis, et Coreopsidis in unum commiscere, ne limites generum tandem evanescant. Heic itaque facies, et natura ceriat cum lege, et arte. Morisonus (\$.5.5.) secutus naturæ filum, inplicat suum ariadneum in Gordios solo gladio explicandos: e. gr. Classe 7. Liliaceis sociantur Anemone, Dryas, Hydrccotyle. 5. Leguminosis .. Oxalis, Fragaria, Epimedium. 9. Compositis ... Eryngium, Bromelia, Cactus. 58. Verticillatis... Asperifolice omnes. I I. Graminibus.... Planiago.

Divisio in Arbores, et Herl as videtur esse aden naturalis, ac in se fallacissima, et lubrica (\$.78.7.)

BOTANICA innititur fixis Generibus, cujus ita progressus hic fuit.

TOURNEFORTICS primus, characteres genericos ex lege artis condidit.

PLUNIIERUS americanas plantas ad genera coëgit.

BOERHAAVilS nonnulla genera addidit.

PETITUS paucissima adje it.

PARISIENSES Academici Marchant, Isnard, Nissole, Condamine actis gallorum varia inserucrunt. 
VAILLANTIUS Botanices reformationem incepit. JUSSI EL Fratres nobis acta parisina ornarunt. RUPPIUS, et DILLENIUS, conjunctissimi socii, in Germaniâ Botanicem reformare allaborarunt.

DILLENIUS, Anglus factus, plurima pulchra elaboravit. PONTEDERA in Italiâ Botanicem expolire tentavit.

MICHELIUS Italicam Botanicam excoluit. BUXBAUMIUS in Oriente pauca genera legit.

AMIMANNUS in Rutheno nonnulla confecit.

HOUSTONUS in americano itinere plurima indagavit, sed periit.

HALLERUS Helvetiæ plantas solidè examinavit.

GMELINUS Sibiriæ plantas, laborioso itinere, primus scrutatus est.

MONTIUS rarissimo genere nuper innotuit.

EGO examinavi hxc omnia genera ad leges artis; characteres reformavi, et tanquam nova condidi. Gronovius, Roysnus, Burmannus plantas obtulere numerosissimas, rarissimas.

\section{TOURNEFORTIANA.}

Abies $\dot{T}$. Larix $\mathrm{T}$. Acanthus $\mathrm{T}$. Acer $\mathrm{T}$. Achillea V. Ptarmica T. Millefolium T. Aconitum T. Actrea L. Cbristophoriana T. Adiantum T. Adoxa L. Moschatellina T. Agrimonia T. Agrimonoides $\mathrm{T}$. Ajuga L. Bugula T. Alchemilla L. Alcbimilla T. Alisma D. R.

Damasonium T. V. Allium T. H.

Moly B.

Cepa T.

Porrum T.
Scorodoprasum. Mich. Aloë T.

Alnus $\mathrm{T}$. Alsine $\mathrm{T}$.

Alyssum $T$. Alyssoides $T$. Vesicaria $T$. Amaranthus T. Amaryllis L. Lilionarcissus T. Ambrosia $\mathrm{T}$.

Ammi $T$. Ammioides B. Amygdalus $\mathrm{T}$. Persica $T$. Anacardium L. Acajou T. Anacyclus L. Cotula T. Santolinoides V. Anagallis T. Anagyris $\mathrm{T}$. Anchusa L. Buglossum T. 
Andrachne I..Telephioides T. Aster T. V. Androsace $\mathrm{T}$. Aretia Hall. Anemone $\mathrm{T}$. Anemonoides D. Anemone-Ranunculus. $D$. Anethum T.

Fceniculum $\mathrm{T}$. Asteropterus V. Astragalus T. Astrantia T. Athamanta L. Meum T. Atriplex T. Atropa L. Belladonna T. Avena $\mathrm{T}$. Anthemis L.Cbamamelum T.V. Axyris L. Ceratoides? T. Anthericum L. Phalangium T. Antirrhinum T.

Linaria $\mathrm{T}$.

Asarina $\mathrm{T}$.

Elatine D.

Anthyllis L. Vulneraria T. Aphyllanthes $\mathrm{T}$. Apium T. A pocynum $\mathbf{T}$. Aquilegia T. Aralia T. Arbutus $\mathrm{T}$.

Uva ursi $T$.

Arctium L. Lappa T. V. Argemone. $\mathrm{T}$. Aristolochia $\mathrm{T}$. Artemisia $T$.

Abrotanum $\mathrm{T}$.

Absinthium T. V.

Arum $T$.

Arisarum $\mathrm{T}$.

Dracunculus $\mathrm{T}$.

Colocasia B.

Aruncus L. Barba capre T. Arundo T.

Asarum $\mathrm{T}$.

Hypocistis $\mathrm{T}$.

Asclepias $\mathrm{T}$. Asparagus T. Asperugo $\mathrm{T}$. Asphodelus $\mathrm{T}$.

Ballota L. Ballote T.

Begonia $\mathrm{T}$.

Bellis T. V.

Bellis Leucanthemum Mick.

Berberis $\mathrm{T}$.

Beta T.

Betonica $\mathrm{T}$.

Betula $T$.

Bidens $\mathrm{T}$.

Ceratocephalus $V$.

Bignonia $\mathrm{T}$.

Biscutella L. Thtaspidium T.

Biserrula L. Pelecinus T.

Borrago $T$.

Borraginoides. B. Cynoglos-

Brassica T.

Rapa $T$.

Napus $T$.

Bromelia Pl.

Ananas $\mathrm{T}$.

Karatas P1.

Pinguin Dilla

Brunella T.

Bryonia $\mathrm{T}$.

Bunias L. Erucago T. Kakile T.

Bunium L. Bulbocastanum T. Buphthalmum $\mathrm{T}$.

Asteriscus T. V.

Asplenium L. Linguacervina T. Anthemis Mich.

Trichomanęs T.

Bupleurum T. 
Butomus T.

Buxus $T$.

Cachrys $\mathrm{T}$.

Cactus L. Melocactus T.

Opuntia T. Tuna D.

Pereskia Pl.

Calendula Rp. Caltha T.

Dimorphotheca V.

Cardispermum Trant.
Celtis T.

Centaurea L. facea T. $V$.

Cyanus $\mathrm{T}, \mathrm{V}$.

Centaurium majus T. V. Calcirrapa V. Raponticum V. Raponticoides $\mathrm{T}$. $\mathrm{V}$. Amberboi V. Crocodilum V. Crupina D.

Calligonum L. Pclygonoides $\mathrm{T}$. Cerastium D. Myosotis $T$.

Caltha L. Populago T.

Campanula $\mathrm{T}$.

Ceratonia L. Siliqua $T$.

Cerbera L.

CamphorosmaL.CamphorataT. Thevetia L. Abouai T:

Canna L. Cannacorus T.

Cannabis $\mathrm{T}$.

Capparis $\mathrm{T}$.

Cercis L. Siliquastrum $T$.

Cerinthe $\mathrm{T}$.

Capsicum $\mathrm{T}$.

Cardamine $\mathrm{T}$.

Cerinthoides B.

Chærophyllum. T.

CardiospermumL،Corindum $\mathrm{T}$, Chelidonium $\mathrm{T}$.

Carduus T. V.

Cirsium T. V.

Eriocephalus V.

Polyacantha V.

Silybum V.

Glaucium T.

Chelone $\mathrm{T}$.

Pentastemon Mitch?

Chenopodium, T.

Chondrilla T. V.

Carex L. Cyperoides T. D.

Carex D.

Carica L. Papaja $P$.

Carlina T. V.

Carlinoides $\mathrm{V}$.

Carpesium L. Conyzoides $T$.

Carpinus $\mathrm{T}$.

Carthamus T. V.

Atractylis $\mathrm{V}$.

Carthanoides V.

Carum R. Carvi $T$.

Chrysanthemum $\mathbf{T}$.

Leucanthemum T. Bellidi-

Chrysosplenium T. oides $V$.

Cicer $\mathrm{T}$. Lens $\mathrm{T}$.

Cichorium T. V.

Circæa $\mathrm{T}$.

Cistus $\mathrm{T}$.

Helianthemum $\mathbf{T}$.

Caryophyllus L.Aromaticus T.

Cassia $\mathrm{T}$.

Caryophyllodendron $V$. Senna. $T$.

Citrus L. Citrum T.

Aurantium $\mathrm{T}$.

Limon $\mathrm{T}$.

Clematis L. Clematitis $T$.

Viticella D.

Catananche V. Catanance $T$. lee L. Sinapistrum $T$.

Caucalis $\mathrm{T}$.

Clinopodium $\mathrm{T}$. 
Clitoria D. Ternatea $T$.

Clypeola L. Fon-Thlaspi $T$.

Cneorlim L. Chamelea $\mathrm{T}$.

Cnicus T. V. Acarna $V$.

Cochlearia $\mathrm{T}$.

Coronopus Rp.

Coix L. Lacryma fob. $T$.

Colchicum T.

Colutea T.

Conium L. Cicuta T.

Convallaria L. Lilium conval-

Polygonatum $\mathrm{T}$.

$\operatorname{lium} T$.

Unifolium D.

Convolvulus $\mathrm{T}$.

Conyza $\mathrm{T}$.

Corchorus $\mathrm{T}$.

Coriandrum T.

Coris T.

Cornus $\mathrm{T}$.

Mesomora Rudb.

Virga sanguinea D. Ossea $\mathrm{Rp}$.

Coronilla T.

Emerus $\mathbf{T}$.

Securidaca $T$.

Corylus T.

Cotyledon T. B. D.

Crambe T.

Rapistrum $T$.

Cratægus $\mathrm{T}$.

Crithmum $\mathrm{T}$.

Crocus T.

Crotalaria $\mathrm{T}$.

Croton L. Ricinoides $T$.

Crucianella L. Rubeola T.

Cucubalus T.

Cucumis $\mathrm{T}$.

Colocynthis T.

Melo T.

Anguria $\mathrm{T}$.
Cucurbita $\mathrm{T}$.

Pepo T.

Melopepo T.

Cuminum $\mathrm{T}$.

Cunila L. Marrubiastrum $T$.

Cupressus T.

Cuscuta T.

Cyclamen $T$.

Cynara V. Cinara $T$.

Cynoglossum $\mathrm{T}$.

Omphalodes $\mathrm{T}$.

Cyperus T.

Cypripedium L. Calccolus $T$. Cytisus $\mathrm{T}$.

Daphne L. Thymelea $T$.

Datisca L. Cannabina T.

Datura R. Stramonium $T_{\text {. }}$

Daucus $\mathrm{T}$.

Delphinium $\mathrm{T}$.

Dentaria T.

Dianthus L. Caryophyllus $T$. Tunica Rp.

Dictamnus L. Fraxinella $T$.

Diervilla T.

Digitalis $\mathrm{T}$.

Diospyros L, M. Guajacana $T$.

Dipsacus T.

Dodartia $T$.

Doronicum T.

Bellidiastrum $\mathrm{M}$.

Dorycnium $\mathrm{T}$.

Dracocephalum $\mathbf{T}$. Moldavica T.

Drosera L. Ros solis $T$.

Echinophora T.

Echinops L. Echinopus T.V.

Echium T.

Elæagnus T.

Elatine T. Alsinastrum T. $\dot{V}$. Potamopithys Buxb.

Em- 
Empetrum T.

Ephedra T.

Epilobium D. Chamenerium T.

Epimedium T.

Equisetum T.

Erica T.

Erinus L. Ageratum $T$.

Eriophorum L. Linagrostis $T$.

Ervum T.

Eryngium T.

Erysimum T.

Erythrina T.Corallodendron T. Erythronium L. Dens canis T. Esculus L. Hippocastanum T. Pavia $B$.

Evonymus T.

Eupatorium T.

Euphorbia L. Tithymalus T.

Tithymaloides T.

Euphorbium Isnard.

Euphrasia T.

Odontites D.

Fagonia T.

Fagus $\mathrm{T}$.

Castanea $T$.

Ferula $T$.

Ficus $\mathrm{T}$.

Filipendula $\mathrm{T}$.

Ulmaria T.

Fragaria $\mathrm{T}$.

Fraxinus $\mathrm{T}$.

Eritillaria $T$.

Corona imperialis $\mathrm{T}$.

Lilio-Fritillaria B.

Fucus T.

Fumaria $\mathrm{T}$.

Capnoides $\mathrm{T}$.

Cysticapnos B. Corydalis D. Cucullaria J.

Galega T.

Galium L. Gallium T.

Aparine T. I.

Garidella T.

Genipa T.

Genista L. Spartium T. Genistella T.

Gentiana T.

Centaurium minus $\mathrm{T}$.

Geranium T.

Geum L. Caryophyllata $T$.

Gladiolus T

Glaux T.

Globularia T.

Alypum Niss.

Gloriosa L. Methonica T. D. Glycyrrhiza T.

Gnaphalium V.

Helichrysum V. Elichry-

Helichrysoides $\mathrm{V}$.

sum $T$.

Filago T. V.

Gomphrena L. Amaranthoides T. Caraxeron V.

Gossypium L. Xylon T.

Gundelia T. Hacub. V.

Hæmanthus T.

Dracunculoides $B$.

Hedera $T$.

Hedysarum $T$.

Onobrychis T. Alhagi $\mathrm{T}$.

Helianthus L. Corona Solis

Heliotropium T. T. $V$.

Helleborus T.

Helxine L. Fagopyrum $T$.

Hemerocallis L. Lilio-Asphodelus $T$.

Liliastrum $\mathrm{T}$.

Heracleum L. Sphondylium. T.

Hermannia $T$.

Herniaria $\mathrm{T}$. 


\section{GENER A.}

Paronychla $T$.

Hesperis $\mathrm{T}$.

Hibiscus L. Ketmia T.

Trionum L. .

Malvaviscus D.

Hieracium T. V.

Pilosella T.V.

Hippocrepis L.Ferrumequir.T.

Hippophaë L. Rhamnoides T.

Hordeum T.

Humulus L. Lupulus T.

Hyacinthus T.

Muscari $\mathrm{T}$.

Hydrocharis L. Morsus Rane

T. Stratiotes D. Mi-

Hydrocotyle T.

croleuconymphrea $B$.

Hydrophyllum $\mathrm{T}$.

Hyoscyamus T.

Hypecoum T.

Hypericum T.

Androsæmim $\mathrm{T}$.

Ascyrum T.

Hysscpus T.

\section{Jasminum $\mathrm{T}$.}

Jatropha L. Manihot T.

Bernhardia Houst.

Ilex L. Aquifolium.T.

Do:lonæa Pl.

Impatiens: R. Balsamina T.

Imperatoria $T$.

Ipomœea L. Quamoclit T.

Volubilis D.

\section{Iris $\mathrm{T}$.}

Xiphium.T.

Hermodactylus T.

Sisyrinchium T. Isatis T.

Jugláns L. Nux $\mathrm{T}$.

Juncus .T.

Juniperus $T$.
Cedrus T.

Sabina B.

Justicia H. Adhatoda T.

Kleinia L. Cacalianthe in un D.

Cacalia T.

Porophyllum V.

Lactuca T. V. .

Lagocia L. Cuminoides T. I

Lamium $\mathrm{T}$.

Lapsana Li. Lampsana V.

Hedypnois T.

Rhagadiolus T. V.

Zazintha T. V.

Rhagadioloides $V$.

Laserpitium T.

Lathræa L. Clandestisa T.

Anblatum T.

Phelypæa T.

Lathyrus $\mathrm{T}$.

Clymenum $\mathrm{T}$.

A phaca $\mathrm{T}$.

Nissolia T.

Lavanduia $T$.

Stoechas $T$.

Lavatera T. D.

Laurus $\mathrm{T}$.

Borbonia P.

Persea P.

Benzoë B.

Leontice L. Leontopetalum T. Leontodon L. Densleonis T. V. Taraxáconoides $\mathrm{V}$.

Leonurus $\mathrm{T}$.

Cardiaca T.

Lepidium $T$.

Nasturtium $T$.

Leućojum Rupp. Narcisso-Lcticojuin $\mathrm{T}$.

Lichen T. Licherioides $D$.

Coralloides D..: K 
Usnea D.

Ligusticum $\mathrm{T}$.

Cicutaria $\mathrm{T}$.

Ligustrum $\mathrm{T}$.

Lilium T. Lirium Roy.

Linum $\mathrm{T}$.

Radiola D. Chamelinum V.

Linocarpon Mich.

Lithospermum $T$.

Lobelia P.

Rapuntium T.

Dortmanna Rudb.

Laurentia Mich.

Lonicera R.

Caprifolium T.

Periclymenum $\mathrm{T}$.

Chamæcerasus $\mathrm{T}$.

Xylosteum T.

Triosteospermum D.

Symphoricarpos D.

Lotus $T$.

Lunaria $\mathrm{T}$.

Lupinus $\mathrm{T}$.

Lychnis $\mathrm{T}$.

Lycoperdum T. Bovista D. Lycoperdoides M.

Lycoperdastrum $M$.

Geaster M.

Tubera $\mathrm{T}$.

Lycopodium T. D.

Lycopodioides $\mathbf{D}$.

Selago D.

Selaginoides $D$.

Lycopsis L. Echioides T. D。

Lycopus $T$.

Lysimachia $\mathrm{T}$.

Lythrum L. Salicaria T.

-Malope L. Malacoides. T.

Malva $\mathrm{T}$.

Althra $\mathrm{T}$.

Alcea $\mathrm{T}$.
Mandragora $T$.

Marrubium $\mathrm{T}$.

Pseudodictamnus T.

Matricaria T. V.

Medicago T.

Medica T.

Melampyrum T.

Melia L. Azedarach T.

Melianthus T.

Melissa T.

Calamintha $\mathrm{T}$.

Menispermum T. D.

Mentha $\mathrm{T}$.

Pulegium R.

Menyanthes $\mathrm{T}$.

Nymphoides $\mathrm{T}$.

Mercurialis $\mathrm{T}$.

Mesembryanthemum D. Ficoides $\mathrm{T}$.

Mespilus T.

Micropus L. Gnaphaloides T.

Mimosa $\mathrm{T}$.

Acacia T.

Inga $P$.

Mirabilis R. Falapa T.

Mitella T.

Moluccella L. Molucca T.

Momordica T.

Luffa.T. D.

Elaterium B.

Monotropa L. Orobanchoides T. Hypopithys D.

Morińa T. Diotheca V.

Morus T.

Myagrum $\mathrm{T}$.

Myrica L. Gale.T. D.

Myrtus T.

Narcissus T.

Neottia L. Nidus avis T.

Corallorhiza Rupp.

Nepeta R. Cataria T. 


\section{GENERA.}

Nerium T.

Nicotiana $T$.

Nigelia $T$.

Nymphæa $T$. Nelumbo T.

Ocymum T.

Oenanthe $T$.

Oenothera L. Onagra T.

Olea T. V.

Ononis T.

Ophioglossum T.

Ophrys T.

Orchis $T$.

Limodorum $\mathrm{T}$.

Origanum $\mathrm{T}$.

Majorana $\mathrm{T}$.

Ornithogalum $\mathrm{T}$.

Stellaris D.

Ornithopus L. Ornithopod. T. Orobanche T. Aphyllon Mitch.

Orobus T.

Oryza T. M.

Osmunda $T$.

Osteospermum L. Chrysanthe-

Monilifera V.

Osyris L. Casia T.

Oxalis L. Oxys T.

Oxyoides Gare.

Pæonia $\mathrm{T}$.

Papaver $\mathrm{T}$.

Parietaria T.

Paris L. Herba Paris T.

Parnassia T.

Passiflora L. Granadilla T. Murucuja T.

Pastinaca T.

Pedicularis $T$.

Sceptrum Carolinum Rudb. Peganum L. Harmala T.
Periploca $T$.

Peucedanum $\mathrm{T}$.

Phaca L. Astragaloides T.

Phaseolus T.

Phellandrium T.

Philadelphus L. Syringa T.

Phillyrea T. V.

Phlomis T.

Physalis L. Alkekengi T.

Phyteuma L. Rapunculus T.

Phytolacca T.

Pimpinella R. Tragoselinum $\mathrm{T}$.

Pinguicula $T$.

Pinus $\mathrm{T}$.

Pistacia L. Terebinthus T.

Lent iscus $\mathrm{T}$.

Pisum T.

Plantago $T$.

Coronopus $\mathrm{T}$.

Psyllium T.

Platanus $\mathrm{T}$.

Plumbago $\mathrm{T}$.

Plumeria T.

Podophyllum L. Anapodophytmoides T. Poinciana T. lum $T$.

Polemonium $\mathrm{T}$.

Polygala $T$.

Penæa P. Chamaluxus T.

Polygonum T.

Bistorta T.

Persicaria T

Fagopyrum T. Helxine L.

Polypodium $\mathrm{T}$.

Populus T.

Portulaca T.

Potamogeton T.

Potentilla L.Pentaphylloides T.

Quinquefolium $\mathrm{\Gamma}$.

Primula L. veris T.

Auricula ursi T.

Prunus T.

$\mathrm{K} \&$ 
Armeniaca $\mathrm{T}$.

Cerasus T. L.

Paáus R. Laurocerasus T.

Psidium L. Guajav.، T.

Pulmonaria $T$.

Pulsatilla T.

Punica $T$.

Pyrola T.

Pyrus T.

Mialus $\mathrm{T}$.

Cydonia T.

Quercus T.

Ilex $T$.

Suber $\mathrm{T}$.

Ranunculus T.

Ranunculoides $\mathrm{V}$.

Ficaria D.

Raphanus T.

Raphanistrum $T$.

Reseda T.

Luteola $\mathrm{T}$.

Sesamoides $\mathrm{T}$.

Rhammus $T$.

Frangula $\mathrm{T}$.

Alaternus T.'

Paliurus T.

Ziziphus $T$.

Cervispina D.

Rheum L. Rhabarbarum T.

Rhinanthus L. Elepbas T.

Crista galli R. D.

Rhododendros L. Chamerrbo- Scilla L. Lilio-byacinthus T.

dodendros 'T. Memacylon M. Rhus T.

Toxicodendron $T$.

Cotinus T.

Rib $=$ s T.

Grossularia T.

Ribesium D.

Ricinus T.
Robinia L. Pseudo-Acacia T. Rosa T.

Rosmarinus $\mathrm{T}$.

Rubia T.

Rubus 'T.

Rumex L. Lapatbum T. Acetosa T.

Ruscus T.

Ruta T.

Pseudo-Ruta Mich.

Salicornia T...

Salix T.

Salsola P. Kali T.

Salvia T.

Horminum $\mathrm{T}$.

Sclarea $T$.

Sambucus T.

Samolus T.

Sanguisorba R. Pimpinella T.

Sanicula $T$.

Santolina T. V.

Baccharis V.

Sapindus T.

Sarracena $T$.

Satureja T.

Thymbra T.

Saxifraga $T$.

Geum $T$.

Scabiosa T.

Scandix $\mathrm{T}$.

Myrrhis T.

Schinus L. Molle T.

Hyacinthus stellaris $\mathrm{R}$.

Scirpus T.

Scolymus T. V.

Scorpiurus I. Scorpioides $\boldsymbol{T}$.

Scorzonera T.

Scorzoneroides V.

Scrophularia T.

Scutellaria R. Cassida T. 
Secale $T$.

Sedum $\mathrm{T}$. Anacampseros $T$.

Selinum L. Oreoselinum $\mathrm{T}$.

Thysselinum $\mathrm{T}$.

Senecio T.

Jacobra $T$.

Serapias L. Hellebarine $T$.

Sicyos L. Sicyoides T. Bryonioides D.

Sida L. Malvinda $D$. Abutilon $\mathrm{T}$.

Sideritis $\mathrm{T}$.

Sinapi $T$.

Sisymbrium T.

Radicula D.

Sisyrinchium L. BermudianaT. Sium $\mathbf{T}$.

Sisarum T.

Falcaria D.

Smilax $T$.

Smyrnium $\mathrm{T}$.

Soianum $\mathrm{T}$.

Melongena $T$.

Iycopersicon T.

Soldanella T.

Solidago V.

Virga aurea T. V.

Doria D.

Sonchus T.

Crepis V.

Sorbus T.

Sparganium $T$.

Spartium L. Genista T.

Cytiso-Genista $\mathrm{T}$.

Spinacia T.

Spiræa $\mathrm{T}$.

Spongia $\mathrm{T}$.

Stachys R. Galeopsis $T$.

Staphylza L. Staphylodendron. Trifolium T.

Statice $\mathbf{T}$.
Limonium $\mathrm{T}$.

Styrax T.

Symphytum T

Syringa R. Lilac. T.

Tagetes T. V.

Tamarindus $\mathrm{T}$.

Tamarix L. Tamariscus $T$.

Tamus L. Tarnnus $T$.

Tanacetum $T$.

Taxus T.

Telephium T: ef mistiust

Teucrium $T$.

Polium $\mathrm{T}$.

Chamædrys T.

Chamæpithys $T$.

Iva D.

Thalictrum $\mathrm{T}$.

Thapsia $\mathrm{T}$.

Theligonum L. Cynocrambe T.

Theobroma L. Cacao T.

Guazuma P.

Thlaspi $\mathrm{T}$.

Bursa pastoris $\mathrm{T}$.

Thuya $T$.

Thymus $\mathrm{T}$.

Tilia T.

Tordylium $\mathrm{T}$.

Tormentilla $\mathrm{T}$.

Trachelium T.

Tradescantia Rup, Ephemerum

Tragacantha $T$.

$T$.

Tragopogon $T$.

Tragopogonoides $T$.

Trapa L. Tribuloides $T$.

Tribulus $T$.

Melilotus $\mathrm{T}$.

Lupinaster Buxb.

K 3

Tri- 
Trifoliastrum Mich.

Triphylloides Pont.

Triglochin L. Funcago $T$.

Trigonella L. Fanum Grec.

Triticum $\mathrm{T}$.

Tropæolum L. Cardamind. T.

Tulipa T.

Turritis $\mathrm{T}$.

Tussilago T. V. Petasites $\mathrm{T} . \dot{\mathrm{V}}$.

Typha $\mathrm{T}$.

Vaccinium R, Vitis idres $T$, Oxycoccos $T$.

Valantia T. Cruciata $\boldsymbol{T}$.

Valeriana $T$.

Valerianella $\mathrm{T}$.

Valerianoides $V$.

Veratrum $T$.

Verbascum $\mathbf{T}$.

Blattaria T.

Verbena $\mathrm{T}$.

Sherardia V.

Blæria $\mathrm{H}_{\text {. }}$
Krmpfera $\mathrm{H}$.

Veronica $T$.

Bonarota M. Pederota $\boldsymbol{L}$.

iburnum T.

Tinus T.'

Opulus $\mathrm{T}$.

Vicia T.

Faba $\mathrm{T}$.

Vinca L. Pervinca $T$.

Viola $\mathrm{T}$.

Viscum T.

Vitex T.

Vitis T.

Ulex L. Genista-Spartium $T$. Erinaceus T.?

Ulmus $\mathrm{T}$.

Urtica $T$ :

Xanthium $\mathrm{T}$.

Xeranthemum T. V. Xeranthemoides V.

Zea L. Mays $T$.

Zygophyllum L. Fabago T.

\section{$P L U M E R I I$.}

Achras I. Sapota P. Alpinia L. Alpina $P$. Annona L. Guanabanus P. Arachis L. Aracbidna P.

Arachidnoides Niss. Ascyrum L. Hypericoides $\boldsymbol{P}$.

Barleria $P$.

Bauhinia P.

Bellonia P.

Besleria P.

Bocconia P.

Bontia P.

Breynia $\mathrm{P}$.

Brossxa? $\mathrm{P}$.
Brunfelsia P.

Bucephalon? P.

Cæsalpinia $\mathrm{P}$.

Calophyllum L. Calaba P.Ca-

Cameraria $\mathrm{P}$. lopbyllodendron $V$.

Chrysobalanus L. Icaco $P$.

Chrysophyllum L. Cainito $P$.

Cissampelos L. Caapeba $P$.

Clusia P.

Columnea $\mathrm{P}$.

Commelina $\mathrm{P}$. Zannonia $\mathrm{P}$.

Cordia $\mathrm{P}$. 
Cornutia P. Agnanthus V.

Crateva L. Tapia P.

Crescentia L. Cuijete P.

Cupania P.

Dalechampia ? P.

Dioscorea P.

Dorstenia P. H.

Duranta? L. Castorea P.

Epidendrum L. Varilla $P$.

Feuillea L. Nhandiroba $P$. Fuchsia? P.

Gerardia P.

Gesneria P.

Guajacum P.

Guidonia P.

Guilandina L. Bonduc. P.

Helicteres L. Isora P.

Hernandia? P.

Hippocratea L. Coa P...W

Hippomane L. Mançanilla? P.

Hymenæa? Courbaril. P.

Sloanea P.

Lantana L. Camara P. Myro-Spondias L. Monbin P.

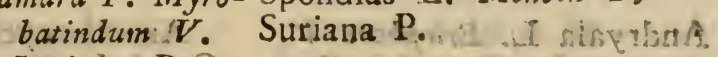
Loranthus V. Lonicera P.

Magnolia $\mathbf{P}$. Malpighia P.

Mammæa L. Mammei P.

Maranta P.

Marcgravia $\mathbf{P}$.

Matthiola 2?: P.

-Mentzelia P.

Morinda $C$ Rojoc, $P$.

Morisona P:

Muntingia $\mathbf{P}$.

Musa P.
Spigelia L. Arapabaca P.

Bihai P.

Ochna L. Fabotapita P.

Oldenlandia P.

Ovieda L. Valdia $P$.

Parkinsonia P.

Paullinia L. Seriana $P$. Cururu P.

Petiveria $\mathrm{P}$.

Piper L.-Saururus $\boldsymbol{P}$.

Pisonia P. Penitagonotbeca V.

Pistia L. Fodda pait. $P$.

Plinia $\mathrm{P}$.

Plucknetia P.

Rajania L. fan-Raja $P$.

Rauwolfia Y.

Renealmia P.

Rheedea L. van Rhcedea $P$. Rhizophora L. Mangles P. Rivina P. Solansides T.

Rondeletia P.

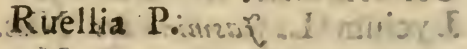

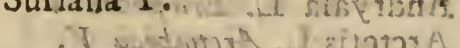

-Tabernxemontana P. psials

Thalia? Lo Cortusav P

Theophrasta L. Eresia. P.

Tillandsia L. Caraguats $P$.

Tournefortia L. ' Pittopial $P$.

-Tragia P.

-Triumfetta P.

Turnera $\mathrm{P}$.

Ximenia? P.

Xylon L. Ceiba. $P$.

\section{4}

BOER- 


\section{BOERHAAVII.}

Acalypha L. Ricinocarpus $B$. Leucadendron L. Hypophyllom Blitum L. Chenopodio-Morus' B. Morocarpus Rupp. Lepidocarpodendrum $B$.

Chrysocoma D. Coma aurea B. Phyllis L. Bupleroides B. Clutia B. Protea L. Conocarpodenitron $B$.

Cortusa B. Scoparia L. Samoloides $B$.

Glechioma L. Chamecissus B. Seseli B.

Glycine L. Apios B. Stratiotes I. Aloides B. Hottonia B. Stratiotes V. Tetragonia L. Tetragonocar-

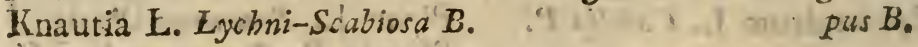

$$
\text { PETITI. }
$$

Acorus L. Calamus aromaticus $P$. M. Calla L. Provenzalia P. Anguina Trew. Isnardia L. Dantia Petit.

\section{'PAIR ISIENSIU M.}

Aizoon L. Ficoidéa Niss. Marchantia March.

Celastrus L. Evonymoides. Isn. Lunularia Mich.

CinchonaL.QuinquinaCondam. Parthenium L. Partheniastrum

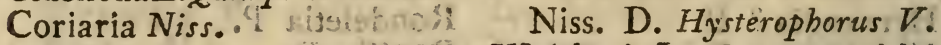
Hycium L. Fasminoides Niss. Waltheria L. Monospermalthiea D. M. 7 ?old. Isn.

\section{$V A I L L A N T I I$.}

Andryala L. Eriophoris V.S Arctotis L. Arctotbeca $V$. Atractylís L. Crocollyloídés $V$. Elephantopus V. D. Boerhavia V.AntanisopbyllumV. Helenia L. HeleniastriumV: Cephalanthus L. Platanocepha- Hyoseris L. Tafaxaconastr. V. Léntodontoides V.

Celosia L. StachyarpagophoraV. Hypochæris V. .

Ceratophyllum L. Hydrocenatopbyllum TVaill: Dichotophyllum D.! sis:11:it

Chara V. Hippuris D. P. Clavaria V. Fungoides D. Co- Najas L. Fluvialis $\boldsymbol{V} . M$. ralloides T. Wh. Dil Onopordum V.I

Cotula V. Achyrophofus $V$ : Iva L. Tarchonanthus $V$. Myriophyllum V. Pentaptera"Spylläm Dobrixo $/ 1$ Othonna L. Jacobrastrum V. Ja- 
Jacobæoides V.

Panax L. Araliastrum V. Panacea Mitch.

Picris L. Helmintotheca $V$. Prenanthes V. Sagittaria L. Sagitta V. D.
Sphranthus V.

Verbesina P. Ceratocephaloid.V. Eupatoriophalacron V. D. Utricularia L. Lentibularia $V$. Zannichellia M. Algoides $V$. Aponogeton Pt.Graminifoliw D.

\section{$\mathcal{F} U S S I \mathbb{E} I$.}

Coffea L. Coffe J.

Neurada J.

CorispermumJ.RhagrostisBuxb. Pilularia J.

$$
R U P P I I \text {, et DILLENII. }
$$

Adonis R. D.

Marsilea M.

Ægópodium L. Podagraria D. Ledum R.

Agaricus $D$.

Amanita D.

Aphanes L. Percepier D.

Boletus D. Suillus $M$.

Polyporus M.

Bromus L. IEgilops $D$.

Bryum D.

Callitriche L. Stellaria D.

Centunculus. D. Anagallidi-

Conferva D.

astruin $M$.

Corrigiola L. Polygonifolia $D$.

Draba D.

Erìgeron L. Conyzella D.

Fontinalis D.

Galeopsis L. Tetrabit $D$.

Hepatica R. D.

Hippuris L. Pinastella D. Lim Scleranthus L. Knazvel $R . D$.

Holosteum D.

$$
\text { nopeuce } V \text {. }
$$

Hydnum L. Erinaceus D.

Hypnum D.

Iberis R. D.

Illecebrum R: Corrigiola $D$.

Jungermannia R. M. Licbena-

- Itwe strum $D$.

Muscoides. M.
Lemna L. Lenticula $D . M T$.

Hydrophace Buxb.

Limosella L.PlantaginellaR.D.

Mnium D.

Montia M. Cameraria $D$.

Myosotis R..D.

Myosurus R. D.

Peplis L. Portu!a D. Glaucoides $M$.

Peziza D. Cyathoides Mr.

hallus M. Morcbella D.

Boletus M.

Phallo-Boletus M.

Polytrichum D.

Saginá L. Alsinella. D. Spergula $R$.

Sempervivum R.

Serratula D.

Sherardia D.

Sperrgula D.

Sphagnum D.

Subularia Raj.

Trientalis R.

Ulva D.

Yucca D. Cordyline Roy. 
DILLENII.

AchyranthesL. AchyracanthaD. Phlox L. Lychnidea $D$. Anacampseros L.Telephiastrum Porella L. Poronia D.

Crassula D.

Eriocephalus D.

Isoetes L. Calamaria D.

Melochia D.

Pancratium D. Patagonula L. Patagonica D. Urena D.
D. Sanguinaria D. Sideroxylon D. Silene L. Viscago D. Spermacoce D. Tetragonotheca D.

Chamærops.L: Chameriphes $\vec{P}$.

Galenia L. Sherardia P. Ageratum L. Carelia $P$.

Anthospermum L. Tournefortia. $P$.

\section{MICHELII.}

Andromeda L. Polifolia Buxb. Frankenia L. Franca M. Ledum M. Chamedaphne Bx. Herminium L. Monorchis M. Anthoceros M. Blasia M. Byssus M. Aspergillus M. Botrytis M. Holcus L. Sorghum M. Marsilea L. Salvinia $M$. Mucor M. Mucilago. M. Lycogala M.

Cenchrus L. Panicastrella M. Orvala L. Papia M. Clathrus M. Riccia M. Cynomorium M. Drypis M.

Elvela L. Fungoides D. Fungoidaster M. Eugenia M. Ruppia L. Bucca ferrea is. Tillæa M. Tozzia M.

Trichosanthes L. Anguina $M$. Valisneria M.

Ceratocarpus B.

$$
B U X B A U M I I \text {. }
$$

$$
A M M A N N I \text {. }
$$

Amethystea L. . Amethystina Pentapetes L. PterospermadenA. H.

Cymbaria A. Gmelina L. Michelia A. dron $A$. Siphonanthus L. Siphonanthemerm $A$. 
Ammannia $\mathrm{H}$.

Bannisteria $\mathrm{H}$.

Budleja $\mathrm{H}$.

Conocarpus L. Rudbeckia $H$.

Gronovia Mart.

Heliocarpus L. Montia $\mathrm{H}_{\text {, }}$

Lippia $\mathrm{H}$.

Loeselia L. Royenia H.
HOUSTONI.

Martynia $\mathrm{H}$.

Milleria $\mathrm{H}$.

Mitreola L. Mitra $\mathrm{H}$.

Petrea H.

Pontederia L. Micbelia $\mathrm{H}$.

Randia? H.

Richardia $\mathrm{H}$.

Volkameria L. Duglassia H.

Cherleria $\mathrm{H}$.

$$
H A L L E R I \text {. }
$$

Stelleria G.

$$
\text { GMELINI, }
$$

Aldrovanda $M$,

$$
\text { MIO NTII, }
$$

NOSTRA.

EUROP压 ASIÆ. AMERIC E. AFRICE. Acrostichum. AdenantheraR.Acnida Mitch. Achyronia. Agilops. AEginetia. Agave. Anastatica Agrostemma, Eschynomene. Amorpha. Antholyza. Agrostis. Allophylus. Arethusa G. ArctopusL.B. Aira. Amomum. Bartsia. Barreria. Alcea. Anabasis. Strebelina H. Blæria. Alopecurus, Antidesma B, Bixa. Althæa. Artedia.

Browallia. Angelica. Anisum. Averrhoa. Buchnera. Avicennia.

Callicarpa. Bobartia. Borassus. Borbonia. Anthoxanthum.Bartramia. Arabis. Basella. Arenaria. Asperula. Cambogia. Atragene. Atraphaxis. Camellia. Azalea. Ceropegia.

Baccharis. Cissus. Sphondylacocc.M.Brabejum. Capraria. Brunia. Catesbæa G. Burmannia. Ceanothus. Caryota. Cestrum.

Chionanthus. Cassine. ClerodendrüB. Chrysogonum, Chironia. Coccus. Briza. Coldenia. Citharexylon J, Cliffortia. Bubon. Connarus. Bufonia S. Bulbocodium. Celsia.

Cornucopix,

Costus. Crinum.
Claytonia. Clethra G. Collinsonia. Coreopsis. Craniolaria. Dalea.
Corymbium.

Corypha. Cynanchum. Diosma. Dracontiuma 
Cicuta Curcuma. Dianthera G. Eranthemum.

Comarum. Cynometra. Diodia G. Exacum.

Coronaria. Delima. Eriocaulon G. Cerbera.

Cressa. Dillenia. "Gleditsia L. Gethyllis.

Cynosurus. Dodonæa. Melilobus M. Barleria.

Dactylis R. Elrocarpus B.Grislea. Gnidia.

Diapensia. Flagellaria. Hrematoxylum. Lachnea R.

Dryas. Garcinia Hamamelis G. Grewia.

Elymus. Hedyotis. Trilopus M. Halleria.

Ethusa. Hugonia Hemionitis. Hebenstretia.

Festuca. Jambəlifera. Heuchera. Hirtella.

Galanthus. Indigophora. Houstonia G. Ixia.

Gratiola. Jussiæà. Hura Kiggelaria.

Gypsophila. Ixora. Hydrangea G. Myrsine.

Horminum. Kæmpferia. Itea G.Diconan-Passerina.

Jasione. Knoxia.

Inula L. Hele-Lawsonia. Limodorum Pharnaceum. gi. M. Penæa. nium V. Melastoma B. R. G. Philyca.

Ischæmum.

Isopy rum. Memecylon.

Lagurus.

Mesua.

Liquidambar. Phœenix.

Lonchitis. Psoralea.

Linnæa G.

Lolium.

Melica.

Melitis.

Michelia.

Liriodendrum. Prasium.

$\begin{array}{ll}\text { Milium. Nepenthes B. } \\ \text { Morhingia. } & \text { Nyctanthes. }\end{array}$

Mimusops.

Ludwigia.

Roëlla.

Medeola. Royena.

Mollugo.

Musænda B. Melampodium. Selago.

Nardus.

Olax.

Melanthium. Sesamum.

Myristica.

MelothriaL.M.Stapelia.

Panicum.

Phalaris.

Phleum.

Роз.

Ophiorhiza.

Mimulus.Cynor-Stoebe.

Polyenemum.

Poterium.

Pteris.

Rhodiola.

Saponaria.

Satyrium.

Ophioxylum.

Pavetta.

rbirchium M. Strehelina.

Sicheuchzeria.

Schœnus.

Phyllanthus. Onoclea. Angio-

Monarda.

Struthia.

Napæa A.C. Tarchonanthus.

Polianthes.

Rumphia.

Nyssa.

Pothos.

Santalum.

Penthris

Saururus I. Polypremum.

Polypremum.

Sigesbeckia:

Sophora.

Sterculia.

Strychnus.

Thea.

Symphoranthus

Prinos G.

Proserpinaca.

Ptelea. 


\section{GENERA.}

Sibbaldi Tomex.

Sison.

Splachnum.

Swertia.

Thesitum.

Vella.

Zostera.
Torenia.

Trewia.

Triopteris.

Vateria.

Uvaria.

Zannonia.
Rudbeckia L.

Qbeliscotbeca V.

Saccharum.

Samyda.

Sauvagesia. . I lis hit.9

Schwalbea G.

Securidaca.

Silphium.

Stewartia. Malacodendros M.

Tetracera.

Toluifera.

Trichomanes.

Trichostema G.

Tridax.

Uniola.

Uvularia.

Xyris G.

Zizania.

Elymus M.
Zizophora.
Hedyosmos M. 


\section{NOM INA.}

210. DENOMINATIO, alterum ( $15 \mathrm{I}$ ) Botanices fundamentum, factâ dispositione ( 152$)$, nomina primùm imponat.

Nomina si nescis, perit et cognitio rerum.

Unicum ubi genus, unicum erit nomen, \$. 215.

Veterum nomina plerumque præstantissima; Recentiorum pejora fuere.

Critica Botanica Denominationem genericam, specificam, et variantem rationibus, et exemplis proposuit; hine de ea in præsenti paucis.

2 I I. Nomina vera plantis imponere, Botanicis (?) genuinis (26) tantùm in potestate est.

Botanicus novit genera distincta, et nomina antea recepta. Idiotæ imposuere nomina absurda.

R. E L I G I O S Á.

Pater noster Cyperus. Christi Oculus Aster

Bonus Henricus Chenopodium. Palma Orchis

Noli me tangere Impatiens. Spina Rhamnus.

Morsus Diaboli Scabiosa

Filius antePatrem Tussilago MariæCalceusCypripedium

Herba Fumana Cistus.

Mater berbarum Artemisia

Surge, et ambula Gentiana

Fuga Damomum Hypericum

Chlanys Alchemilla

Stragula Gálium

Veneris labrum Dipsacus

Umbilicus Cotyledon

Calceus Cypripedium Pecten Scandix

Jovis Barba Sempervivum.

Non Sistematici Botanici,

Bontiania Pt.

Breyniana Pt.

Ruyschiana Pt.

Drakena Clus. Dorstenia.

212. Nomina omnia sunt in ipsâ vegetabilis enunciatione vel Muta, ut Classis ( I6o), et Ordinis (I6I); vel Sonora, ut Genericum (I59), Specificum ( 157 ), et Varians (158).

Nomen omne plantarum constabit nomine Generico, et Specifico. 
Nomen classis, et ordinis nunquam intrabunt nomen Plantæ , sed subintelligantur; hinc Royenus, qui Lilium pro nomine classico adhibuit, rectè exclusit idem in genere, et substituit terminationem græcam Lirlum.

213. Quæcunque plantæ genere conveniunt $\left(16_{5}\right)$, eodem nomine generico (2I2) designandæ sunt.

Citrus T. Aurantium T. Limon T. Pyrus T. Malus T. Cydonia. T.

214. Quæcunque, è contrarià (2 13$)$, plantæ genere differunt (166), diverso nomine generico, $\left(2{ }_{3}\right)$ designandæ sunt.

Consolida

major.

media

minor.

minima

rubra

aurea

regalis

Delphinium

sarracenica. Salidago.

palustris, Comarum.
Trifolium

arborescens Cytisus

acetosum $\mathrm{Oxalis}$

corniculatum Lotus

falcatum Medicago

Fragaria

Hepatica

Menyanthes

Trifolium

Fagonia.

215. Nomen genericum in eodem genere $\left(2 I_{3}\right)$ unicum erit:

Aconitum cæruleum, s. Napellus.

salutiferum, s. Antbora.

Aquifolium, s. Agrifolium.

fasminum, s. Geselmininum.

216. Nomen genericum in edem genere idem erit. Asclepias T. Vincetoxicum Hk. Hirundinaria R. Limosella L. d. Plóntaginella. D. Menyanthoides V. Hottonia B. Stratiotes V. Myriophyllum R. Tetrabit D. Ladanün R. Cannabina B. Radiola D. I- Linoides R. Cbamelinum V.

217. Nomen genericum unum (215), idemque (216), ad diversa designanda Genera assumtum, altero loco excludendum erit. 
Aconitum T. Aconitum Cameraria Pl. Caméraria.

- - - R. Helleborus. _ - _ D. Montia.

Asclepias T. Asclepias Sherardia D. Sherardia.

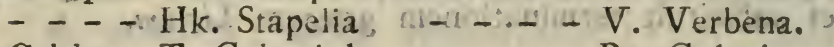

Caltha T. Calendula - $-\mathrm{Pn}_{\text {. Galenia. }}$

-+- Rp. Caltha.

2r8. Quir novum Genus constituit, eidem nomen etiam imponere tenetur.

Nec Cacalia, nec Cacaliastrum; an Titbymaloides Kl. Methonica folio planka Plk.

Ánonymos.

219. Nomen genericum immutabile figatur, antequam specificum ullum componatur.

Nomen spêcificum, sine generico, est quasi pistillum sine campanâ §. 286 .

220. Nomina generica primitiva nemo sanus introducit.

Barbara vocabula omnia sunt nobis, uti primitiva, cùm lingua eorum $a b$ eruditis non intelligitur.

Dubia vocabula plantarum, de quibus vix constet è qua, linguâ orta sunt:

Osmunda, Tanacetum.

221. Nomina generica ex duobus vocabulis integris, ac distinctis facta, è Republicâ Botanicâ releganda sunt.

Bella donna Trus Atropa.

Centaurium majus T. Centaurea.

Corona Solis T. Helianthus.

Crista Galli D. Ri Rbinantbus.

Dens Leonis T. Leontodon.

Vitis idra T. Vucciniam.

222. Nomina generiça ex duobus vocabulis latinis integris, et conjunctis composita, vix toleranda sunt.

Ejusmodi vocabula græcấ linguâ pulcherima sunt; at Latina non facilè eadem admittit;

Comaurea. Chrysocoma. 
Admisimus nonnulla vocabula latina, sed non ideo in posterum imitanda sunt, uti:

Cornucopice Sch.

Rosmarinus $\mathrm{T}$.

Sempervivum R.

Sanguisorba $\mathrm{R}$.

223. Nomina generica ex vocabulo græco, et latino, similibusque hybrida, non agnoscenda sunt: : Barbaro-Latina:

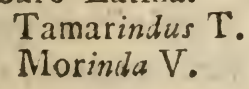

Græco-Latina:

Cardaminlum $\mathrm{T}$.

Chrysanthemindum V. Sapindus T.

224. Nomina generica ab uno vocabulo plantarum generico fracto, altero integro composita, Botanicis indigna sunt. ArisArum T. Arum.

CannAcorus T. Canna. Greed admittend.

CapnOrchis B. Fumaria. ElaAgnus. Olex Vitex. LilioHyacinthusT.Scilla. Ciss Ampelos.HederæVitis. LinAgros is 'T. Eriophorum. LizuroCérasus T. Padus.

225. Nomen genericum, cui syllaba una, vel altera prǽponitur, ut aliud planè genus, quàm antea, significet, excludendum est.

Acri-Viola.B. Tropocolum. Chame DrysT. Teucrium. Bulbo-CastanumT.Eunium. ChimePithys T. .m. Teucriun. Cyno-Crambe T. Thel:gonum.PseudoDictamnusT.M.arrubium. Chame-NeriumT. Epilobium. PseudoOrchis M. Orchis. fon-Thlaspi T. rlypeola. PseudoRuta M. Rut.. Leuco-NymiphraB.Nymphre.

Micro Nymphæa B. Ficta?

Micro-Leuco-Nymphra B. Hydrocharis.

226. Nomina generica in oides desinentia e foro Botanico releganda sunt. Agrimonoides T. Agrimonia. Valerianell-OidesB.Valeriana. Alyssoides T. Alyssum. Alsin-Aitr-Oides $\mathrm{Kr}$. Asteroides T. Buphthalmum.Jon-Thlaspi-Oides Kr. Astragaloides T. Phaca. 
Chrysanthemoides T. Osteospermum.
Cuminoides T.
Cyperoides $\mathrm{T}$.
Nymphoides $\mathrm{T}$.
Lagecia.
CapnOides R. Fumsria. Carex. Menyanthes.
Pentaphylloides T. Potentilla.
Rhamnoides $\mathrm{T}$.
Ricinoides $\mathrm{T}$.
Hippophaë
Croton.
Telephioides T.
Tribuloides $\mathrm{T}$.
Andrachne.
Trapa.

227. Nomina generica ex aliis nominibus genericis, cum syllabâ quadam in fine additâ, conflata non placent.

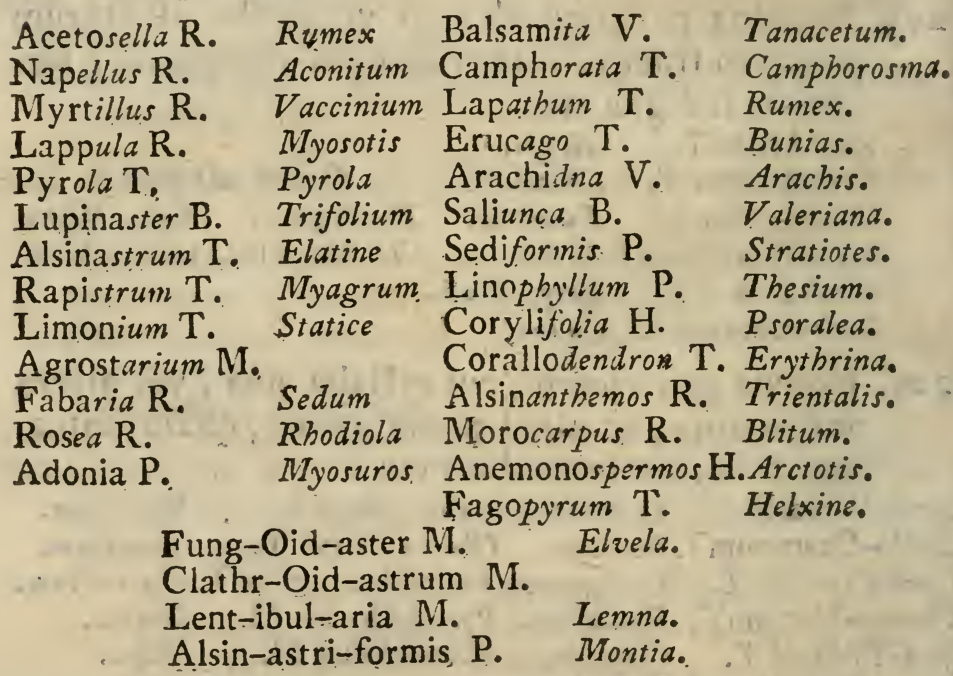

228. Nomina generica, simili sono exeuntia, ansam præbent confusionis.
Alsine $\mathrm{T}$. Alsine
Alsinoides $\mathrm{Rj}$.
Alsinella D.
Bufonia
Juncus $T$.
Juncus.
Scirpus T. Scirpus.
Alsinastrum $\mathrm{T}$.
Sagina
Cyperus $\mathrm{T}$.
Cyperus.
Elatıne
Juncoides $S$.
Funcus.
Alsinastroides $\mathrm{Kr}$.
Alsinastriformis $\mathrm{Pk}$.
Cameraria
Scirpoides S. Carex. Alsinanthemos $\mathrm{Rj}$. Trientalis
Cyperoides S. Carex. 
Alsinanthemum Kr. Alsine Juncoidi afinis S. Scheuchzeria. Cyperoidi affinis $\mathrm{S}$. Juncago T. Triglochis.

Lycogala M. Junco aftinis S. Schocnus. Lycopersicon T. Solanum Pseudo-Cyperus S. Schocnus. Lycoperdon T. Lycoperdon.Scirpo-Cyperus S. Scirpus. Lycoperdastrum M.

Licoperdoides M. Nymphra T. Nymphaa. Lycopodioides D. Lycopodium Nymphoides T. Menyanthes. Lycopodium D. Lycopodium-Micro-Nymphæa B. Lycopus T. Lycopus Leuco-Nymphæa B. Nymphea. Lycopsis R. Lycopsis Micro Leuco-Nymphæa B. Hydrocharis.

229. Nomina generica, quæ ex græcầ, vel latinâ linguâ radicem non habent, rejicienda sunt.

\section{Germanica}

Datura R. Turcarum.

Bovista D. Itycoperdon Ketmia T. Syrorum Hibiscus. Beccabunga R. Veronica Albagi T. Arabuin Hcdysarum. Brunella Prunella. Ribes R. Arabum

Anglica

Doronicum T. Arabum.

Percepier D. Aphanes. Tenga Malabarorum.

Gallica.

Orvala L.

Hispanica. Adbutoda T. Zeylanensium Justicia. Sesban Egyptiorum.

Fabotapita P. Americanorum Ochna. Sarsaparilla. Smilax. CaapebaP.BrasilianorumCissampelos Scorzoniera T.

Italica.

Galega. T.

Quasimodogenita assumimus nomina Ḃarbara, dum vocabula excludenda nova reddimus, formatả é linguầ græcâ,aut latinâ.

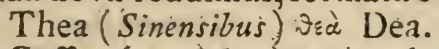

Coffea (Arabibus) rew: ob obmutesco.

Musa (Arabibus) Musa Anton.

Cassine (Americanis) xasjuco machino.

Annona (Anona Amer.) a messe.

Mammea (Mammei Americ.) a fructu mammoso.

Chara (Gallis) Xa.jò gaudium aqux.

Pothos (Zeylanis) iso.

Basella (Malabaris) Basium Cosmet.

Datura (Turcis) vid. Hort. cliff.

Cheiranthus (Keiri arab.) $\chi$ हi९ manus. 
Tambolifera (Jambolines Indis).

Toluifera (Tolu Indis).

Indigofera (Indigo).

Ziziphora Morison.

Gratis recepta, quæ in se Barbara, methodo præcedenti

forte redintegrari possent, e. gr.

$\begin{array}{ll}\text { Bixa O. } & \text { Hura L. } \\ \text { Genipa T. } & \text { Urena D. } \\ \text { Guajacum T. } & \text { Santalum L. } \\ \text { Tulipa T. } & \text { Tucca T. } \\ \text { Liquidambar B. } & \text { Curcuma L. }\end{array}$

230. Nomina generica plantarum, cum Zoologorum, et Lithologorum nomenclaturis communia, si a Botanicis postea assumta, ad ipsos remittenda sunt.

... Quaćrupedia.

- Taxus T. Meles. Lapides.

Elephas T. Rhinanthus. Granatum. Punica.

Erinaceus D. Hydnum. Hyacinthus T.

Onagra T. Oenothera. Heliotropium T. Aves.

Malybdrena Plumbago T.

Acanthus - Fringilla.

Ampelis Vitis.

Lagopus Trifolium.

Meleagris Fritillaria.

Oenanthe Motacilla.

Phalaris Emberiza. Sol. R.

Cælestia.

Amphibia. Iris $\mathrm{T}$.

Natris. K. Ononis.

Pisces.

Buglossum T. Anchusa.

Terrestria.

Hippoglossum Ruscus.

Delphinium $T$.

Pastinaca T. Raja.

Insecta.

Ephemerum T. Commelina P.

Eruca T. Brassica T.

Locusta R. Valeriana. T.

Phalangium. Anthericum.

China.

Molucca T.

Stachas T.

Ternatea $\mathrm{T}$.

Helianthus.

Cinchona. Moluccella. Lavandula. Clitoria. 
Ricinus T. Acarus.
Scolopendrum T. Asplenium. Impatiens R.

Sphondylium T. Heracleum. Patientia Rumex.

Staphylinus R. Daucus T. Concordia Agrimonia. Vermes.
R.alanus
Nepenthes.

23r. Nomina generica cum Anatomicorum, Pathologorum, Therapeuticorum, vel Artificum nomenclaturis communia, omittenda sunt. Anatomica.

Auricula Hk. Primula. Candela Rhizophora.

Clitoris Br. Clitoria. Sagitta Sagittaria.

Epiglottis Kn. Astragalus. Serra Biserrula.

Priapus A. Nepenthes. Muscipula Silene.

Unibilicus H. Cotyledon. Corona Helianthus.

- Pathologica.

Paralysis Primula.

Soda "Salsola.

Sphacelus Salvia.

Canara Lantana.

Bursa Thlaspi.

Verruca Lapsana.

Therapeutica.

Ptarmica T. Achillea.

Cardiaca T. Leonurus.

Hepatica R. Hepatica.

Vesicaria T. Alyssum?

Vulneraria $\mathrm{T}$. Anthyllis.

Solaa equina Hippocrepis.

232. Nomina generica contraria speciei alicui sui generis mala sunt.

Chrysanthemum flore albo. Pilosella glabra.

Cyanus luteus. Holosteum est molle.

Convolvulus erectus. Unifolium est diphyllum.

233. Nomina generica, cum Classium, et Ordinum $\mathrm{Na}$ turalium nomenclaturis communia, omittenda sunt.

Fungus.

Alga.

Muscus.

Filix.

Palma.

Lilium.
Planta.

Arbor.

Frutex.

Suffrutex.

Herba.

Vegetabile.

L. 3

No- 
234. Nomina generica Diminutiva,et è Linguâ Latinâ contorta, quamvis non præstantissima, toleranda sunt.

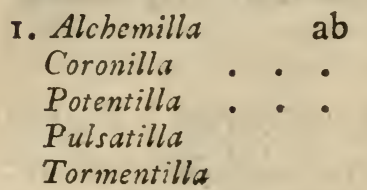

2. Basella

Biscutella

Crucianella

Hirtella

Limosella

Mitella

Moluccella

Nigella

Pimpinella

Porella

Soldanella

Tremella

Trigonella

3. Corrigiola

Gratiola

4. Clypeola

Rbodiola

Uniola

Medeola

Mitreola

5. Pyrola

6. Phaseolus

7. Gladiolus.

8. Samolus

9. Tropeolum

I0. Asperula

Bisserrula

Calendula

Campanula

Crassula

Ferula

Filipendula
Alchemistis Ros foliorum.

Coronato corymbo.

Potentiâ virium.

Pulsatione floris vento.

Torminibus dysentericis.

Basiis fucatis.

Scuto duplici fructûs.

Cruciatis foliis situ.

Hirsutie ramorum.

Limo natali.

Mitrâ, figurâ fructûs.

Moluccis insulis natali.

Nigredine seminum.

Bipinnatis foliis.

Poris vasculorum.

Soldo nummo.

Tremore gelatinoso.

Trigonâ corollâ.

Pedum ligatione.

Gratiâ medicinali.

Clypeo fructûs.

Roseo odore radicis.

Unione glumarum.

Medea, s. Medicâ vi.

Mitrâ, figurâ fructûs.

Pyro, foliorum figurâ.

Phaselo seminis.

Gladio, figurâ foliorum.

Samo insulâ.

Tropæi insigniâ, figurâ.

Asperitate plantæ.

Biserrato fructu.

Calendis omnibus floret.

Campanâ, figurâ corollæ.

Crassitie foliorum.

Feriendi usu.

Filo pendent radices. 
Lavandula

a

Patagonula

Primula

Pinguicula

Sanicula

Serratula

Spergula

II. Ranunculus

Convolvulus

Humulus

12. Asparagus

I3. Asperugo

Mollugo

14. Borrago

Medicage

Plantago

Plumbago

Selago

Solidago

Tussilago

15. Pastinaca

Proserpinaca

Securidaca

Portulaca

16. Reseda

Rubia

Lactuca.

Urtica

Lantana

Spinacia

Salsola

Salix

Salvia

Sedum

Ledum

Lamium

Funcus

Cornus

Juglans

Panicun

Balneis usualis.

Patagoniâ, loco natali.

Præcocitate florum.

Pinguedine foliorum.

Sanando vulnera.

Serratis foliis.

Sparsione seminis.

Ranis cohabitantibus.

Convolutione caulis.

Humo solo.

Asperitate plantæ.

Asperitate plantæ.

Mollitie plantæ.

Cor agens veteribus.

Medis advecta.

Planta tangenda.

Maculâ plumbeâ impositâ.

Silego?

Solidando vuinera.

Tussis domatione.

pastinando loco.

proserpendo.

securi, fructûs figurâ.

Portula.

resedando dolores.

rubedine.

lacte plantæ.

urendo tactu.

lentore ramorum.

spinis fructûs.

salsedine plantæ.

saliendo excrescendo.

Salvando.

sedendo in rupibus.

lædendo odore.

lamiâ larvatâ.

jungendo vimine.

cornu duritie fructûs.

jovis glande.

pane. 
235. Nomina generica Adjectiva Substantivis pejora sunt, adeoque non optima.

I. Arenaria

Convallaria

Clavaria

Capraria

Cochlearia

Coriaria

Coronaria

Crotalaria

Craniolaria

Cymbaria

Dentaria

Flagellaria

Fragaria

Fritillaria

Funnaria

Globularia

Herniaria

Lunaria

Matricaria

Obolaria

Pulmonaric

Parietaria

Persicaria

Sagittaria

Sanguinaria

Saponaria

Scoparia

Scrophularia

Scutellaria

Stellaria

Subularia.

Utricularia

Uvaria

Uvularia

2. Eriophorum

Ecbinophora $\mathbf{T}$.

$R$ bizophora

Ziziphora Moris.

Jambolifera

Toluifera

a

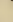

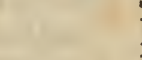

Solo natali.

Loco.

Facie.

Usu alimentorum.

Figurâ foliorum.

Usu œconomico.

Usu œcoisomico.

Figurâ Leguminum.

Figurâ fructûs.

Figurâ fructûs.

Figurâ radicis.

Figurâ foliorum.

Fragrantiâ.

Facie petalorum.

Fumo quasi terræ.

- Figurâ floris.

Usu Medico.

Figurâ fructûs.

Usu Medico.

Figurâ foliorum.

Usu Medico.

Loco.

Persicæ foliis.

Figurâ foliorum.

Succo sanguineo.

$\mathrm{Vi}$ foliorum.

Usu œconomico.

Sctophis medicinæ.

Figurâ calycis.

Figurâ florum.

Figurâ foliorum.

Appendicum radicis figura.

Figurâ fructûs uvæ.

Figurâ inflorescetstiæ uvulæ.

Lanâ fructûs.

Fructu echinato.

Ramis radicatis.

Zizi indis.

Jambolo.

Balsamo Tolu. 
Indigofera

3. Clitaria

Imperatoria

4. Hepatica

5. Scabiosa

Passerina

Angelica

Impatiens.

Gloriosa

Mirabilis

Ametbystea

Pedicularis

Trientalis .

Digitalis

Fontinalis

Turritis

Sempervivum

Momordica -

Bistorta

Saxifraga

Sanguisortix.

Passiflora.

Moluccella

Athamanta

Parnassia.

Marrubium

Smyrnium

Nepeta

Arethasa

Arabis

Punica

Santolina

Thapsia

Colchicum

Cerasus

Samolus

Agaricus

Iberis

Patagonula

Carica

Ligusticum ab

Indigo colore.

Figurâ corollæe.

$\mathrm{Vi}$ radicis.

Figurâ foliorum.

Usu medicinali.

Figurâ .seminis.

vi radicis, seminisque.

elasticitate fructûs.

eximio flore.

diversicoloribus floribus.

colore florum.

vi medicâ.

magnitudine plantæ.

figurâ corollæe Digitalis.

Loco in fontibus.

alta, et angusta.

sempiterno virore.

seminibus quasi masticatis.

Figurâ radicis.

Loco, petrarum rupturis.

vi Miedicà.

Passionis instrumentis?

a Loco.

a

Moluccis insulis.

Urbe Thessaliæ.

Monte Parnasso.

Oppido Italiz.

Urbe Smyrnâ.

Oppido Italix.

Urbe Syrix.

Arabià Regione.

Urbe Carthagine.

Reg. Aquitanix.

Africa oppido.

Colchide, urbe Armeniæ.

Urbe Cerasunte.

Samo insulâ.

Agariâ, urbe Sarmatiæ.

Iberiâ Regione.

Patagoniâ, regione Americes.

Cariâ, Regione Asire.

Liguriâ, Italiæ Regione. 
236. Nomina generica non abutenda sunt ad Sancto. rum, Hominumve in aliâ arte Illustrium favorem captandum, aut memoriam conservandam.

Unicum Botanicorum præmium; hine non abutendum est. SANCTORUM.

Herba Sancti.

Sanctæ

Alberti .... Arabis

Barbarce.... Erysimum.

Antonii .... Epilobium

Benedicti .. Geum.

Christophori. Actæa

Catharine .... Impatiens.

Clare..... Valeriana.

Gerardi... Ægopodium Maria .... Tanacetum

Georgii ... Valeriana Othilice .... Delphinium.

Guilielmi .. Agrimonia Rosæ ..... Pæonia.

Facobi ... Senecio

THEOLOGORUM.

Fohannis.. Hypericum Scriana PI. Paullinia.

Kunigundis. Eupatorium Uvedalia Pet. Osteospermum.

Ladislai . Gentiana Levisanus Pet.

Laurentii . . Sanicula

ILLUSTRIUM.

Pauli .... Primula .I Phelypeea T. Lathræa.

Petri .... Parietaria Bucca ferrea M. . Ruppia.

Philippi... Isatis

Quirini ... Tussilago

Ruperti ... Geranium

Simeonis... Malva

Stephani. . Circrea

Valentini .. Prenia

Zacharia ... Centaurea

Bonarota M. . . Veronica.

237. Nomina generica Poëtica, Deorum ficta, Regum consecrata, et Promotorum Botanices promerita, retineo.

POETICA in veterum eruditione trita:

$\begin{array}{ll}\text { Ambrosia } & \text { Adonis } \\ \text { Nepentbes } & \text { Crocus } \\ \text { Cornucopiae } & \text { Centaurea } \\ \text { Protea } & \text { Cbironia } \\ \text { Actre } & \text { Acbillea } \\ \text { Narcissus } & \text { Prenia } \\ \text { Hyacinthus } & \text { Cerbera }\end{array}$

Amaryllis Canna

Phyllis Syringa?

Circad Smilax

Medeola Mentha

Andromeda Myrsine.

Daphre 
DEORUM :

Asclepias

Mercurialis

Hymenea

Medicorum

MIusa

tonep :anaiprer

Serapias \#gypti

Satyriuin Libidinis

Satureja Satyrus

Sterculea Sterquilinii

Ixora

Malaba rorum

Tagetes.. Jovis nepos.

REGUM.

Eupatorium Ponti (Mithridates) Artemisia Mausoli.

Gentiana Illyrix

Lysimachia Sicilix Althea Oenei.

Telephium Misixe

Teucrium Troja

Helenia

Menelai.

Valeriana

Carlina.

Philadelphus Agypti

Pharnaceum Ponti.

PROMOTORUM.

Borbonia (Gasto) Sr. Begonia . Præfectus.

Eugenia Princeps Poinciana. Præfectus.

Bignonia Abbas Præf. A. P.

Petrea Lord Petre.

Sherardia Consul Smirnx

Cliffortia J. U. D.

Steruartia Comes

Maurocenia Senat. Venet.

Bosea Senat. Lips.

USURPATORES.

Nicotiana J. Nicot.

Einchona.

Euphorbia.

238. Nomina generica, ad Botanici optimè meriti memoriam conservandam constructa, sanctè' servanda sunt.

Hoc unicum, et summum præmium laboris, sanctè servandum, et castè dispensandum ad incitamentum, et ornamentum Botanices. 
Rationes petendæx ex Criticâ Botanicá.

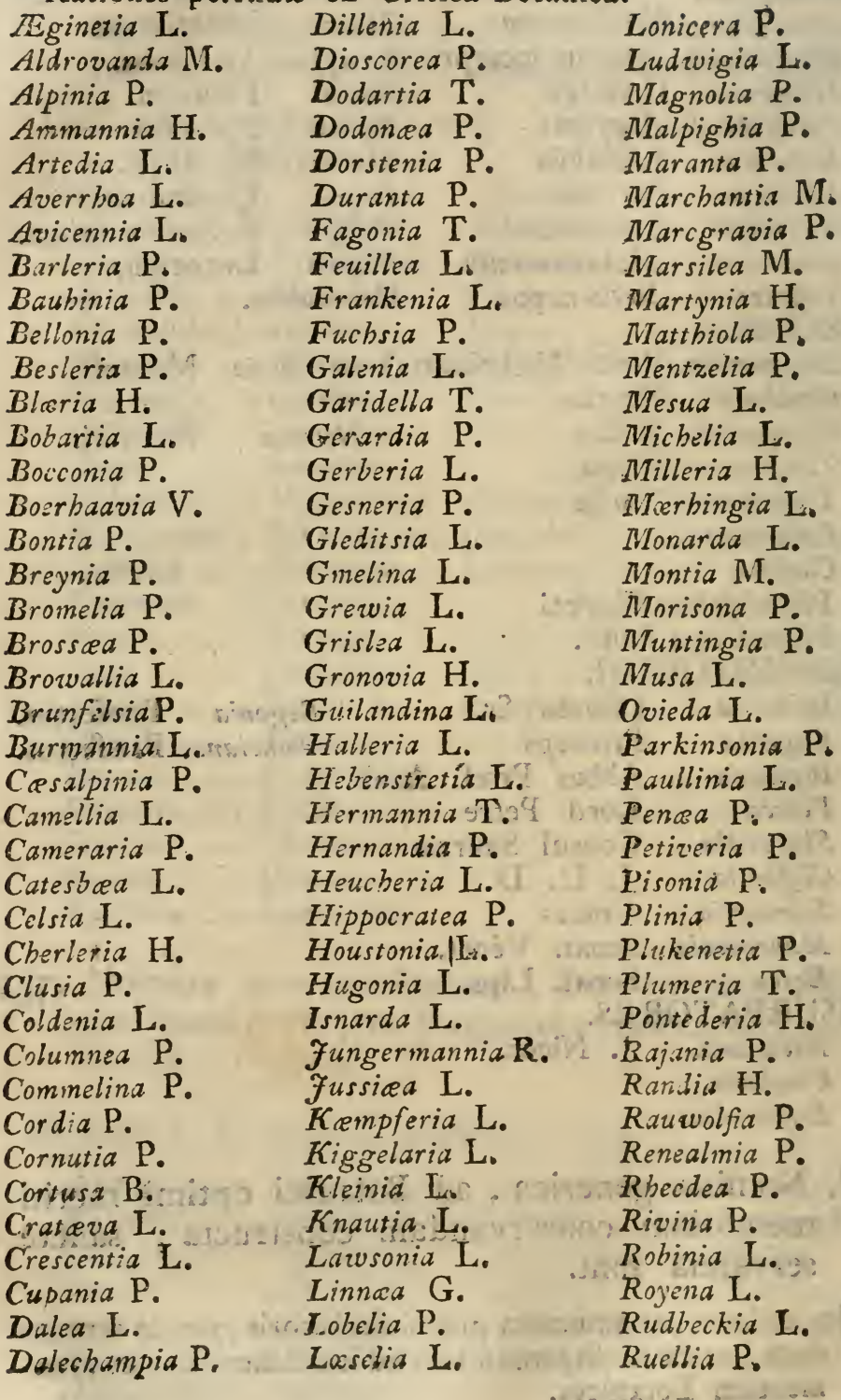




$\begin{array}{lll}\text { Ruppia L. } & \text { Suertia L. } & \text { Triumfetta P. } \\ \text { Siuvagesia L. } & \text { Taberncemontana P. } & \text { Turnera P. } \\ \text { Scheucheria L. } & \text { Thalia P. } & \text { Valantia T. } \\ \text { Sherardia D. } & \text { Theophrasta P. } & \text { Vateria L. } \\ \text { Sibbaldia L. } & \text { Tillaa M. } & \text { Vallisneria M. } \\ \text { Sigesbeckia L. } & \text { Tillandsia. L. : } & \text { Volkameria L. } \\ \text { Sloanea P. } & \text { Tournefortia P. } & \text { Waltheria L. } \\ \text { Spigelia L. } & \text { Tradescantia R. } & \text { Ximenia P. } \\ \text { Stebelina L. } & \text { Tragia P. } & \text { Zanichellia L. } \\ \text { Stapelia L. } & \text { Trezia L. } & \text { Zannonia L. } \\ \text { Suriana P. } & & \end{array}$

INVENTORUM.

Collinsonia L.

Claytonia L

Diervilla $\mathrm{T}$.

Nicotiana $\mathrm{T}$.

Torenia L.

Sarracena? T.

Bartramia L.

Stellera G.

PEREGRINATORUM.

Banisteria H. Virginia.

Bartsia Surinami.

Brunia Orientis.

Clutia Barbaria.

Garcinia Indiæ.

Gundelia Orientis.

Knoxia

Lippia

Mitchella

Oldenlandia

Sarracena

Torenia

Barreria
COMMENDATA.

Budlejz H.

Justicia H.

Richardia H. Richardsan.

Sclavalbea G. Medicus.

Lavatera $\mathrm{T}$.

Morina $\mathrm{T}$.

Hottonia B.

Tozzia M. Abbas curios. Blasia M. Monach. Bo:an.

Riccia M. Senator Eques. Exclusa.

ChomeliaL. SpeciesRondeletiæ. Pavia B. - - - Esculi. Bonarota M. - - Veronicæ. Buccaferrea M. Comes. Franka Medicus.

Laurentia Prof. Med.

Puccinia Prof. Anat.

Salvinia Prof. Græe.

Targionis Medicus,

239. Nomina generica, quæ citra noxam Botanices imposita sunt, ceteris paribus, tolerari debent.

Noxia sunt, qux dedimus $\oint .214-217 \cdot 220-233 \cdot 236$.

Noxia nomina vocamus ea, qux. 
I. Contraria generi \$.215.216.217.

2. Malè constructa $\$ .220-229$.

3. Malè imposita \$. 231.232 .233 .236$.

LATINA obscura, quorum fontes ignoramus, vel qux origine dubia evádunt, assumenda sunt; sed non imitanda: e. gr.

\begin{tabular}{|c|c|c|c|}
\hline Abies & Cucumis & Malva & Scirpus \\
\hline Acer & Cucurbita & Milium & Secale \\
\hline Allium & Cunila & Opulus & Solanum \\
\hline Alnus & Equisetum & Panicum & Sorbus \\
\hline Apium & Ervum & Papaver & Tamarix \\
\hline Aralia & Esculus & Paris & Tilia \\
\hline Arbutus & Ficus & Pinus & Triticum \\
\hline Arundo & Genista & Pisum & Ulmus \\
\hline Atriplex & Hedera & Populus & Ulva \\
\hline Avena & Ilex & Porruin & Verbena \\
\hline Bellis & Illecebrum & Prunus & Veronica \\
\hline Berberis & Inula & Quercus & Viburnuin \\
\hline Betula & Iris & Ros $\alpha$ & Vicia \\
\hline Carduus & Funiperus & Rosmarinus & Vinca \\
\hline Carex & Laurus & Rubia & Visla \\
\hline Carpinus & Ligustrum & Rubus & Viscum \\
\hline Centunculus & Lilium & Rumex & Vitex \\
\hline Cicer & Linum & Ruscus & Vitis \\
\hline Cicuta & Lolium & Salicornia & \\
\hline Cotula & Lupinus & Sambucus & \\
\hline
\end{tabular}

GR IECA obscura, quamplurima eruuntur difficillimè, et eruta dubia tamen persistunt.

$\begin{array}{llll}\text { Achras } & \text { Celtis } & \text { Cuminum } & \text { Gossypium } \\ \text { Aloë } & \text { Cenchrus } & \text { Cycas } & \text { Hibiscus } \\ \text { Amomum } & \text { Cissus } & \text { Cytisus } & \text { Isatis } \\ \text { Anagyris } & \text { Cistus } & \text { D.zucus } & \text { Itea } \\ \text { Aparine } & \text { Citrus } & \text { Dorycnium } & \text { Lathyrus } \\ \text { Atraphaxis } & \text { Cneorum } & \text { Elatine } & \text { Lemna } \\ \text { Blitum } & \text { Coccus } & \text { Elvela } & \text { Lichen } \\ \text { Boletus } & \text { Coix } & \text { Epimedium } & \text { Lotus } \\ \text { Borassus } & \text { Colutea } & \text { Erinus } & \text { Lycium } \\ \text { Byssus } & \text { Comarum } & \text { Eryngium } & \text { Lytbrum } \\ \text { Cactus } & \text { Corylus } & \text { Exacum } & \text { Malope } \\ \text { Cassia } & \text { Costus } & \text { Fucus } & \text { Melia } \\ \text { Cerasus } & \text { Cratogus } & \text { Geum } & \text { Melica } \\ \text { Carum } & \text { Croton } & \text { Glaux } & \text { Melochia } \\ & & & \end{array}$




$\begin{array}{llll}\text { Memecylon } & \text { Phleum } & \text { Samyda } & \text { Statice } \\ \text { Mespilus } & \text { Phlomis } & \text { Scandix } & \text { Strychnus } \\ \text { Morus } & \text { Phonix } & \text { Scilla } & \text { Styrax } \\ \text { Myrica } & \text { Piper } & \text { Sesamum } & \text { Tamus } \\ \text { Mlyrtus } & \text { Pistacia } & \text { Seseli } & \text { Taxus } \\ \text { Nardus } & \text { Platanus } & \text { Sicyos } & \text { Thalictrum } \\ \text { Nerium } & \text { Polemonium } & \text { Sida } & \text { Thesium } \\ \text { Ochna } & \text { Pothos } & \text { Sinapis } & \text { Tridax } \\ \text { Oryza } & \text { Prasium } & \text { Sisymbrium } & \text { Thuya } \\ \text { Pentapetes } & \text { Prinus } & \text { Sium } & \text { Ulex } \\ \text { Pentborum } & \text { Ptelea } & \text { Spartium } & \text { Vella } \\ \text { Peplis } & \text { Rlamnus } & \text { Sphagnum } & \text { Xyris } \\ \text { Peziza } & \text { Rhus } & \text { Spirea } & \text { Zea } \\ \text { Phaca } & \text { Saccharum } & \text { Spongia } & \text { Zizania. } \\ \text { Phillyrea } & & & \end{array}$

Perversa ex erroneâ Lectione veterum, varia metamorphosin insignem subierunt.

Agrimonia pro Argemonia. Betula ... Betulla. Ajuga - - - - Abiga. Equisetum . Equiselis. Aquilegia - - Aquilina. Betonica - - - Vetonica, Myrsine . . Myrsinum P. Borrago - - - Corago. Melotrhia . Melothron P.

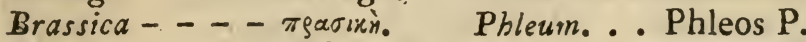

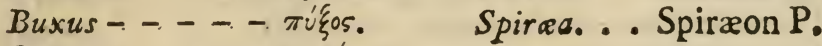
Cuscuta - . - xasítas. Coriandrum- - - Corianum.

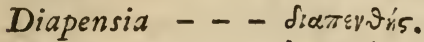

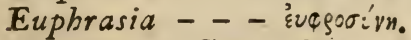
Gomphrena Gromphena P. Lupulus - . - Upulus. Malope ...- $\mu \alpha \lambda \alpha \chi^{\text {n. }}$ Melocbia - - - $\mu \nu \lambda^{\prime} \chi^{n_{0}}$. Thya, malè Thuja, et Thuya. Pimpinella - - - Bifennula. Santolina - - Sanctolina. Spinachia - - Spanachia. Tanacetum - - Athanasia? Verbascum - - Barbascum. Verbesina - - Forbesina. Veronica - - Vetonica. 
240. Nomina generica, quæ Characterem Essentialem, vel Habitum plantæ exhibent, optima sunt.

Etymologia Græca plantarum difficillimè eruitur in plerisque plantis, adeoque conjecturæ sæpius satisfaciunt.

Character Essentialis rarò in plantis obtinetur, licèt optimus.

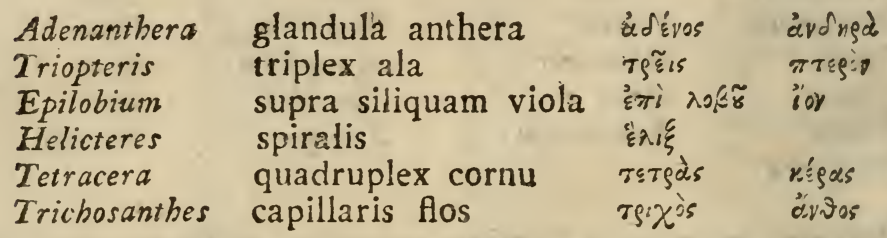

Habitus indicat similitudinem, qua excitatur idea, et ex ideâ nomen: E. Glycyrrbiza
Ophiorrbiza

2. Clerodendrum

Epidendrum

Leucadendrum

Liriodendrum

Rbododendrum

3. Hematoxylum

Opbioxylum

Sideroxyluin

4. Eriocaulon

Caucalis

5. Calopbyllum

Caryophyilum

Ceratophyilum

Cherophyllum

Chrysoptyillum

Hydropbyllum

IVyriophyllum

Podopbyilum

Triphyllum

Zygopbyllum $\left(\begin{array}{l}\text { Radix } \\ p^{t} y^{\prime} \alpha\end{array}\right)$ - Dulcis

(Arbor ) - Fortunata

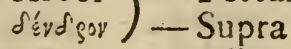

Alba

Liliacea

Rosacea

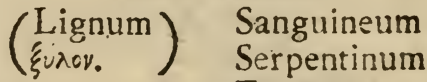
Ferreum

(Caulis) Lanatus

Recubans

Pulchrum

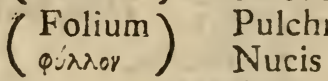

- Cornutum

- Lætum

Aureum

Aquaticum

Infinitum

- Pedatum

Ternatum

Conjugatum youxis

opis

x入กัต०ร.

$\dot{\varepsilon} \pi i$.

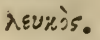

$\lambda \varepsilon: \rho$ :N.

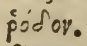

$\tilde{\delta}, i \mu e_{0}$.

¿Q15.

oí̛́ngos.

है ८००.

"'w Ambr.

rancs.

xagia.

$x: \rho \alpha 5$.

$x$ aiga.

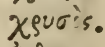

uेंe?.

pugios.

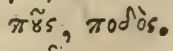

TรEิร.

रuròs.

Cbry- 
6. Chrysocoma.

7. Amarantbus

Cephalanthus

Cheiranthus

Chionanthus

Dianthus

Galantbus

Hemanthus

Helianthus

Loranthus

Melianthus

Phyllanthus

Rbinanthus

Sclerantbus

Siphananthus

Spberanthus

Tarcbonantbus

b. Achyranthes Apbyllanthes I.Tenianthes Nyctanthes

Polianthes

Prenanthes

Trichosantbes

c. Cerintbe

Oenanthe

d. Antbosantbum

e. Melanthium

f. Chrysantbemum

Eranthemum

Mesembryanthemum

Xeranthemum
( $\left.\begin{array}{l}\text { Summitas } \\ x^{\prime} \mu n .\end{array}\right)$ Aurea

xpuris.

(Flos

¿.yงos
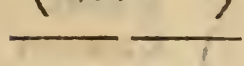

Capitatus

Manualis

Niveus
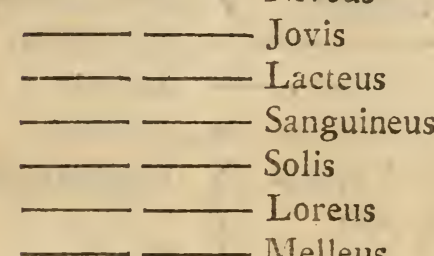

Loreus

Melleus

Foliorum

Nasutus

Exsuccus

Tubulosus

Globosus

Tarchon arabis

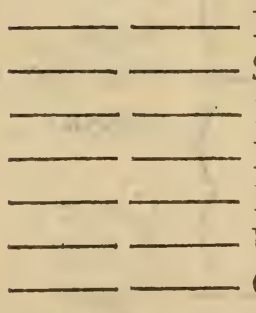

Paleaceus

Sine foliis

¿xugar

aquinos

Menstruus

$\mu$ in

ขं's

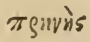

Tó̀is

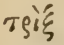

xทควัร

oivn

वैy vos

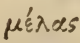

xpuròs

Terræ

Medio germinis $\mu^{\prime} \sigma c s, \varepsilon^{2} \mu \rho_{\rho}^{\prime} j^{\prime} y$ Aridus हnisंs. 


\begin{tabular}{|c|c|c|c|}
\hline 8. Tricbostema & Stamen & Capillare & शji \\
\hline 9. Adenanthera & Anthera & Glandulosa & 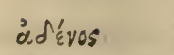 \\
\hline Diantbera & 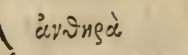 & Duplex & dis \\
\hline 10.Ceratocarpus & Fructus & Cornutus & $x^{\prime} \Leftrightarrow \rho a s$ \\
\hline Conocarpus & xagròs & Strobilaceus & xã̀os \\
\hline Elceocarpus & & Olivaceus & 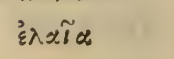 \\
\hline Heliocarpus & & Solaris & n̈rios \\
\hline Callicarpa & & Pulcher & xexios \\
\hline II.Tetragonotheca & $\begin{array}{l}\text { Capsula } \\
\text { ฟัֻ่n }\end{array}$ & Quadrangula & 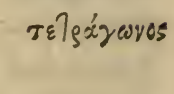 \\
\hline 12. Antbospermum & Semen & Floris & áy-sos \\
\hline Cardiospermum & $\sigma \pi \varepsilon \rho \mu \alpha$ & Cordis & rassia \\
\hline Corispermum & & Cimicis & xógis \\
\hline Lithospermum & & Lapideum & $\lambda i$ ins \\
\hline Menispermum & & Lunatum & $\mu_{i}^{\prime} y_{\prime \prime}$ \\
\hline Osteospermum & & Osseum & ostoy \\
\hline I 3.Diospyros & Granum & Jovis & Siis \\
\hline Isopyrum & $\pi$ ugis & Simile & ioos \\
\hline Melampyrum & & Nigrum & $\mu^{\prime} \hat{\prime} \lambda x s$ \\
\hline I4.Chrysobalanus & Drupa & Aurea & xpuois \\
\hline I 5. AEgilops & Facies & Capræ & al \\
\hline Echinops & $\because 415$ & Echini & 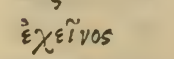 \\
\hline Mimusops & & Simix & $\mu \mu \tilde{\omega}$ \\
\hline Coreopsis & - & Cimicis & xógis \\
\hline Galeopsis & & Felis & 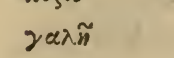 \\
\hline Lycopsis & -1 & Lupi & aúros \\
\hline I6. Bucephalum. & ( Caput & Bovis & Bั̌ร \\
\hline Dracocephalum & $(x \varepsilon p \alpha \lambda \dot{n}$ & Draconis & $\delta \rho \dot{x} x \omega=$ \\
\hline Eriocephalus & & Lanatum & ¿ \\
\hline I7.Leontodon, & (Dens & Leonis & $\lambda \varepsilon \hat{\varepsilon} \omega$. \\
\hline
\end{tabular}




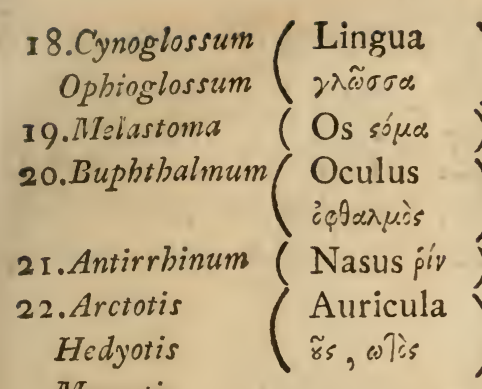

Myosotis

23.Tragopogon

\section{Callitriche}

Polytrichum

24. Anthoceros

Tetracera

25.Cynomorium

26.Cynometra

27. Alopecurus

Cynosurus

Lagurus

Leonurus

Myosurus

Saururus

Scorpiurus

Hippuris

28. Calligonum

Chrysogonum

Polygonum

Theligonum

29.Polyonemum

30. Arctopus

Elephantopus

( Capillus

( Penis $\left(\begin{array}{l}\text { Barba } \\ \pi c ́ g \omega v\end{array}\right)$

Cornu

(x'egas

( Vulva

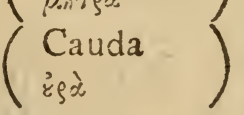

Canis

Leporis

Leonis

Mliris

Lacertæ

Scorpionis

Equi

Pulchrum

Aureum

Multiplex

Femineum

Multiplex

Ursi

Elephantis xécor

opis

$\mu \dot{\lambda} \lambda \times 6$

อชร์

वेगरे

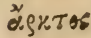

iंरंडs

peús

тgárys

rexis

Toris

อ้ำง

тยтรа́⿱一⿻上丨

xंขอ

rن́wr

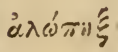

xiver

$\lambda \alpha \gamma$ cos

$\lambda$ '́cov

Nis

odipgs

oxogtrios

i $7 \pi 05$

xa入̀े

x९uròs

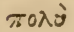

จกักข

mode

\%ектx

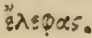




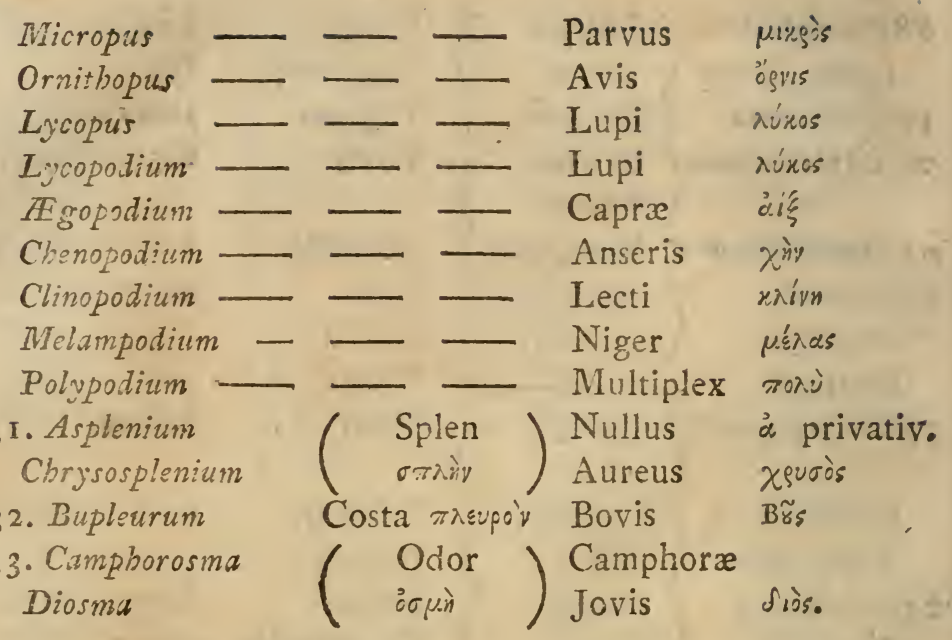

2. ANIMALIA plantarum vocabula suppeditantia.

\begin{tabular}{|c|c|c|c|c|}
\hline Leontice & Leo & $\lambda \operatorname{ls}^{\prime} \omega y$ & & \\
\hline Arctium & Ursus & ä $\rho$ राos & & \\
\hline Cynara & Canis & rijesy & & \\
\hline Cynancbum : & Canis & xicos. & strangulo & ${ }^{5} y \chi_{0}^{0}$ \\
\hline Apocynum & Canis: & xuvis. & abs & वेंगे \\
\hline Lycoperdon & Lupus & núxos. & perdo & $\pi \xi \rho \delta 0$ \\
\hline Onopordon & Asinus & óvos. & perdo & 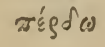 \\
\hline Ononis & Asinus & o'vos. & & \\
\hline Hippopñä & Equi & $i \pi \pi 05$. & splendor & Q⿻áes \\
\hline Hipposnane & Equi & $i \pi \pi 05$. & Furor & Mavix \\
\hline Sisyrincbium & Suis & ข้ร. & Rostrum & p'úr\%os \\
\hline Hyoscyamus & Suis & ös, ข̈os. & Faba & xúapos \\
\hline Hyoseris & Suis & vos. & Lactuca & 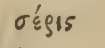 \\
\hline Orobus & Bovem & Ex̃s. & Excito & 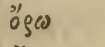 \\
\hline Tragxcantha & Hirci & 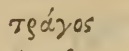 & Spina & $\alpha^{2} x \arg \cdot \vec{\gamma}$ \\
\hline Hieracium & Accipiter & $i \leqslant \rho \alpha_{\xi}^{b}$. & & \\
\hline Geranium & Grus & $y: \rho$ aros & & \\
\hline Chelidoniam & Hirundo & $x^{\varepsilon \lambda i \delta \text { के } y}$ & & \\
\hline Struthia & Passer & รรุ̧จిio\%. & & \\
\hline
\end{tabular}




\begin{tabular}{|c|c|c|c|}
\hline Dracontium & Draco & Spóxoy. & 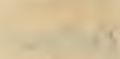 \\
\hline Echium & Vipera & $\chi^{15}$ & \\
\hline Chalone & Testudo & $\chi^{\varepsilon} \lambda^{\prime} \dot{u} y n_{0}$ & \\
\hline D:lpbinium & Delphinus & 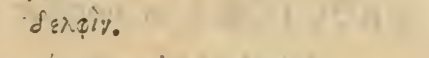 & \\
\hline Coris & Cimex & xógls. nil & 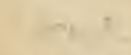 \\
\hline MIelittis th & Apis & $\mu^{\prime} \Omega_{\lambda} \lambda \tau \tau x_{0}$ & \\
\hline IYragrum & Muscas & pivix. & àrsiva. \\
\hline Astragalus & Vertebra & ásgáxycios. & went \\
\hline Acorus & Pupilla & privativum & $x_{0}$ \\
\hline Ophrys & Supercilium & èsus. & , $\quad=$ \\
\hline Oröbis ... & Testiculus & 's $x_{15}$. & is \\
\hline Phallus & Penis & $\varphi \alpha \lambda \geqslant$ & w 11 \\
\hline Cotyledon & Cavitas & rotír., & \\
\hline Splacbnum & Viscus & 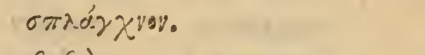 & \\
\hline Bubon 77 & Inguren & Fof $\dot{\omega} v . \quad$ exly & \\
\hline Pleris & Ala & 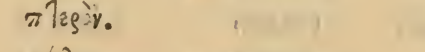 & 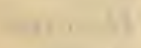 \\
\hline Lythrum & Cruor & 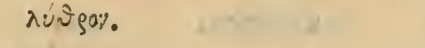 & \\
\hline
\end{tabular}

3. INSTRUMENTA pro similitudine assumta in nomine.

Gompbrena Clavis ligni róugeos

Brabeium Sceptrum $\beta_{\rho \alpha \varepsilon \varepsilon i o v}$

Atractylis Fusum äreaktos

Lonctbitis Lancea tór $\chi^{n}$

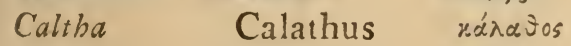

Cercis Spathula xegris

Prinos Serra $\pi \rho^{\prime} \omega \omega$

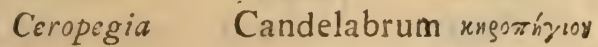

Iychnis Lucerna dúxyos

Phlox Flamma cicis

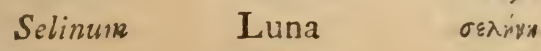

Cestrum Malleus xésga

Sideritis Ferrum oidnpos

Stratiotes Exercitus ssatis

Oenothera Vini civas

Otbonna Linteum อง ช́rn

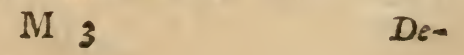


Delima Lima

Lagaccia

Leporis

$\lambda a y$ ess

rubile

Oกพบวร

4. STRUCTURA in nomine usurpata.

Agave Admirabilis èavis

Adoxa gloria o o'sce

Apharies non apparens áquins

Adintum Madefacio diaive

Cleome claușa viśsoug

Clixthrus cancelli

FEscbyromene contraho $\quad " / \%$

Mimosa Mimus mutabilis

Misnulus Mimus personatus

Silene

Ascyrum spumosa $\sigma \varepsilon r i ?$

Holosteum totum ònos.

Asarum vinctum osigoy.

Erytbrina ruber

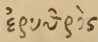

Erytbronium ruber

Aizoon

Semper

Ageratum

Senectus

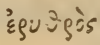

बेहो

Bulbocodium Bulbus

gripos

Aspbodelus

subplanto

for Bòs

Bryonia

germino

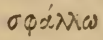

Bryum

germino

Esia

Acantbus

Spina

Egiem

Dolichus

longus

ข้xavฟx

Schinus

findo

Xylon

Lignum

Sori $x$ is

oxi?

$\xi: \lambda \leq y$

Clemutis

viticula.

Periploca

circum

$x \lambda \cdot \mu \alpha$

Scrocnus

Funis

Osyris

ramosa

$\pi i \rho i$

nexus

privativum $\quad a$.

privativum $\alpha_{0}$

pudefio ai $\chi^{i}$ ivoual

privativum

osseum

$\infty$ òs ใ่อง.

privativum

vivum

¿ผ่⿰า

Lana

xक́dros

privativum $\quad \alpha_{c}$

oxoี่ขร

iदugis

Tomex - Tousentum

Gnaphalium

Tomentum

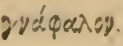




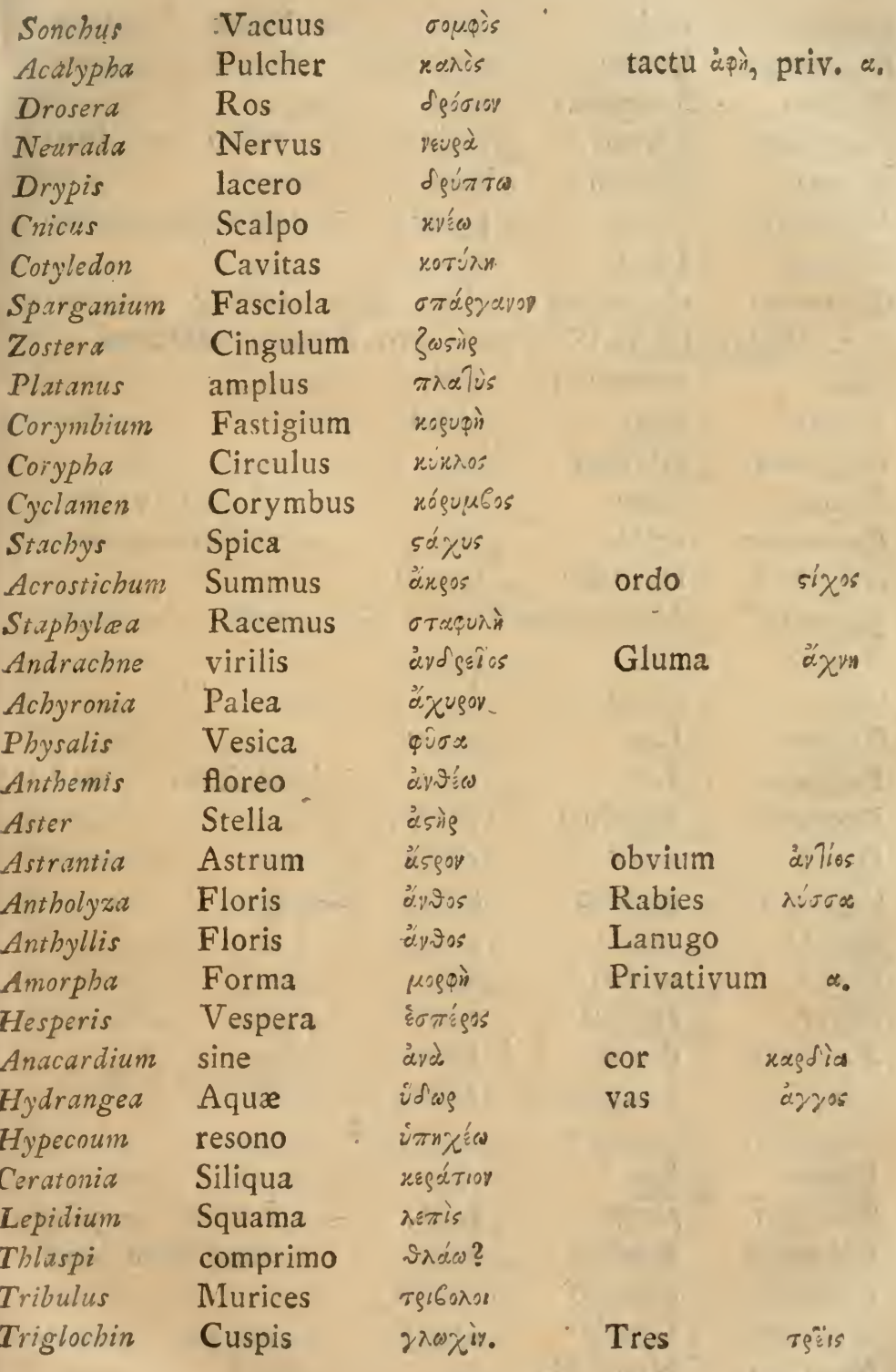


Erigeran

Eriophorum

Phalaris

Tordylium

Elymus

Raphanus

Selinum

Chancerops virgultum ing

हैंวข

qúdios

Togròs

Èर्́co

gafies

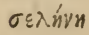

९ंेे
Senex

fero

volvo irs.w

appareo qaíves

5. MEDICINALIS effectus pro Nomine adhibitus.

Alcea

Altbea

Erysimum

Panax

Pancratium

Heracteum

Jairopio

Bromus

Olax

Galium

Polygala

Poterium

Draba

Capsicum

Glycine

Oxalis

Picris

Xantbium

Lapsana

Carthamus

Rbeum

Corcborus

Iscbamum

Peganum

Aristolocbia

Narcissus remedium

sano

salvifico

omne

ormis

Hercules

Medicamentũ iatgòr

Esca

Sulcus

Lac

- Lac

Poculum

acris

mordeo

dulcis

acidus

amarus

flayus

purgo

purgare

fuo

purgo

reprimo

congelo

Lochia

Narce P1. ठतरnे

वे $\lambda)^{\prime}$

¿gंú

$\pi \tilde{a} y$

$\pi \tilde{a} s$

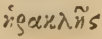

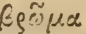

तิ่ $\lambda x$

yetice

yánes

Totrígior

déán

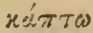

raunis

ơुजs

Tikgìs

gáytos

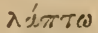

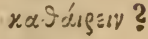

ค'śc

Hog $\omega$,

ixw,

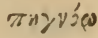

$\lambda \circ \chi \tilde{\varepsilon} \tilde{l \alpha}$ humile $\quad$ aruxi. résws

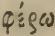




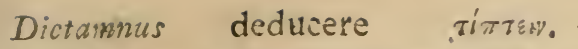

Horminum impetu feror igisasw.

Thymus

Animus ivis.

Symphytum connasci oupoziv.

Holcus

Tractio

¿xiris.

Partbenium

Virginea

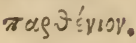

Conyz.z

Scabies

Argemone

Ulcus oculi

xvं $\{\alpha$.

Alyssum

Rabies

Rbexia

Raptura

$\lambda \cup \sigma 50 \%$,

Antidesma

vineulum

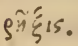

Caibnanche violentia

Anisum

inæequalis

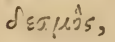

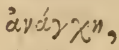

àvíos.

Trachelium

Collum

Anchusa

fucare

Pbytolacca

Pigmentum

Ainaguallis

rideo

Tfisymos.

น้นนันธรเ\%.

Brima

dormio

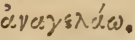

Dipsacus

siiio

Alism.z

anxietas

Butomus

scindens

Helleborus constringo

Egijo.

fițes.

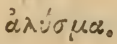

Toùs,

Bovem

$\alpha v \tau_{i}$

contra

$\kappa \% \tau \alpha$

Planta

C: ToY

Orobanche strangulo

हंतें $\omega$,

व̈ $\chi \omega$,

Pabulum

Eัธร

Pbellandrium Suber

Orobum

Eogè

Trichomanes Mania

Hypericum Imago

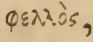

Mavia,

हineेv,

Hominis àd giov

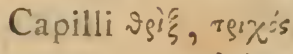

supra

थाँे

6. SOLUM natale in nomine receptum.
Ephedra
Aqua

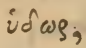
supra
$\varepsilon \pi \ell$
Origanum
Montis
ogos,
letitia
yx́yos
Aconitum
Cautis
¿̇хónn.
Crambe
Siccus
xqúp.Bos.
Azalea
arida
Bunias
Coltis
a $2 x \lambda \fallingdotseq 05$.
Eunium
Collis
Ery:s.
Eryòs. 


\begin{tabular}{|c|c|c|c|c|}
\hline Empetrum & Saxum & 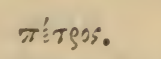 & in & $\varepsilon^{\prime}$ \\
\hline Gypsopbila & Gypsi & rítos, & Amica & $\varphi$ \\
\hline Ammi & Arena & öpups. & & \\
\hline Agrostis & Ager & ärsos. & & \\
\hline Cichorium & Ager & $\begin{array}{l}\text { yosio\%, } \\
\text { sos }\end{array}$ & vado & ríw \\
\hline $\begin{array}{l}\text { Diodia } \\
\text { MIonotropa }\end{array}$ & $\begin{array}{l}\text { subviatica } \\
\text { Solus }\end{array}$ & $\begin{array}{l}\text { Si:d } 105 \\
\text { Hóvos }\end{array}$ & verto & $\tau \rho^{\prime}=\omega \omega$ \\
\hline Latbrax & Clandestina & 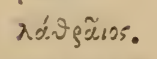 & & \\
\hline Manilragora & Stabulum & prív $\rho е$. & & \\
\hline Anthericum & Flos & $\ddot{d y} \exists c 5$, & sepium & pinyoss \\
\hline Alsine & Lucus & ¿"xoos. & & \\
\hline Hyirocharis & Aqua & $\approx \delta \omega \rho$, & gratia & xígis \\
\hline Hydrocotyle & Aquæ & $i \delta \omega \rho$, & Vas & HOTUג \\
\hline Typha & Palus & rịos. & & \\
\hline Potamogeton & Fluvius & motauss, & cinus & $\gamma \varepsilon i \tau \omega \nu$ \\
\hline Pistia & Alveus & 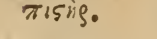 & & \\
\hline
\end{tabular}

7. VARIA, et Miscellanea, unde nutuata fuere nomina.

\begin{tabular}{|c|c|c|c|c|}
\hline Dodecatbeon & Dii & จेड़े, & duodecim & $\delta$ ód हx: \\
\hline Theobroma & Deorum & จะกัด & Cibus & 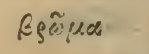 \\
\hline Ambrosia & mortalis & Egstòs, & privativum & $a_{0}$ \\
\hline Eaccharis & Bacchus & fóryos. & & \\
\hline Cypripedium & Veneris & 'x'ssis, & Calceus & $\pi$ ofioy \\
\hline Fasione & Dei & olós p. Vsós, & viola & is \\
\hline Neottia & Pullus nidi. & veotios, & & \\
\hline Cucubalus & jactus & Bór.os, & malus & xaxis \\
\hline Evonymus & Nomen & övolua, & bonum & घंis \\
\hline Hemerocallis & Dies & $\dot{i} \mu^{\prime}=\rho \alpha$, & pulchra & $x \alpha \lambda$ डे \\
\hline Helintropium & Sol & sinios, & vertere & $\tau \rho \rho^{\prime} \approx \varepsilon \varepsilon \nu$ \\
\hline Anilryala & Viri & 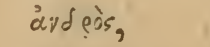 & erratio & $\dot{d} \lambda \dot{n}$ \\
\hline Androsace & Viri & àró gòs, & Scutum & od́xos \\
\hline Allopbyllus & alienigenus & 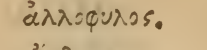 & & \\
\hline AEtbusa & Mendica & $u_{1}, \vartheta_{8 \sigma \alpha}$ & & \\
\hline Isoëtes & Annus & हैं & similis & iros \\
\hline fasminum & Violæe & BOH, & Odor & 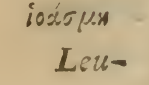 \\
\hline
\end{tabular}




\begin{tabular}{|c|c|c|c|}
\hline Leucnjum & Viola & iov, & alba \\
\hline Ifomu:x & Convolvuli & it, imis, & Similitudo \\
\hline Microcos & Coceus & & parvus \\
\hline Calla & Palearia galli & $x\{\lambda, \alpha|\sigma\rangle$. & $6=$ \\
\hline Arachis & Noxa & a. $\rho \dot{\alpha}$. & C.ti, \\
\hline Arum & Noxa & a.gd. & privativum \\
\hline B.zllota & jacio & $\Leftrightarrow \alpha \lambda, \lambda \omega$. & \\
\hline Phyteum & $\xi$ & Qut บ. & \\
\hline & & & \\
\hline Hemionitis & lus & & \\
\hline
\end{tabular}

Cuchrys

Hordeum tostum rxypis.

Card.zmine Nasturtium xifjauov

Glechomx. Pulegium

Hedysarum Unguenti

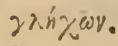

Fraxinus Sepimentum $43 \%$ žsis.

Hypocharis Porcus

Chondrilla Grumus

xoipss, diminutivum ixi

Ornithogalum Lac

Peucedanum Picea

Caryota

Nux

Conium

Pulvis

Erica

frango

Hamamelis Malus

Anemone

vento

Cratagus

robur,

Cirdamine

Cor

Doronicum? munus

Fagus

edere.

Lotus

dulcis

Ocymum

cito (germinans),

Scandix

acus pastoris

Polypremum

multus

$x^{\text {svo gos. }}$

ráxa,

$\pi$ ז'un,

xर्́gosv.

norí.

\& $\rho \equiv x_{0} \omega$.

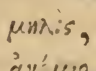

aे $v^{\prime} \mu 10$.

x९xtas.

xभ́,

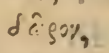

Qxरriv.

$\lambda$ धंтि口.

cixícos.

oxivsig

noris;
Suavitas if íc $\mu$ o

Avis

ogerivas

humilis

d'áuas

simul

ă $\mu \alpha$

domo

victoria

Sopsis.

vinn.

truncus $\pi \varsigma^{2} \mu \nu v$ s. 
241. Vocabula plantarum, veteribus usitata, leguntur vel Grefa apud Hippecraten (H.), TyroPHRASTUM (T.), DiosCORIDEM (D.), vel LATINA apud Plinium (P.), Geoponicos, et Poëtas. AUCTORITAS rocum petenda est a Patribus $\$ .9$.

GRIECA a Grxcis usitata vocabula.

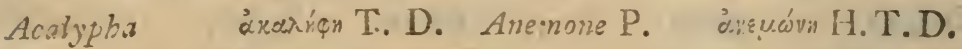
Acanthus P. V. äray-os T. Anirachne P. a.vógdzyxH.T.D. Acbillea P. àximsios T. D. Androsace P. ävfó́raxis D. Acbras. à $\chi \rho \dot{\alpha} \mathrm{D}$. Anisum P. ăviso: D.

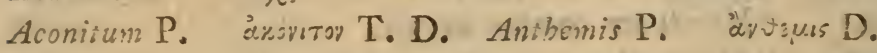
Aoorus P. äroggr D. Anthericum P. ävs şixis T. D.

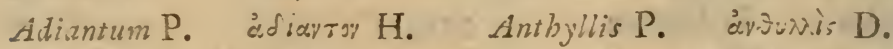

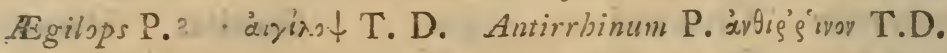

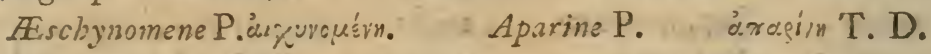

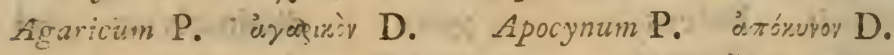

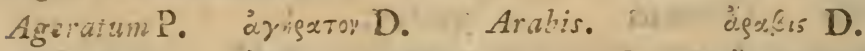
Agrostis. äressis T. D. Arachos P. dexzos T. Aira $\mathrm{P}$. âre. H. T. Arctium P. a.६รтเรу D. Aizon $\mathrm{P}$. as:'çov T,D. àr.x'śs D. Alce. P. cis $x=5 \mu x$ D. Alisma $\mathrm{P}$. cixón D. - Arum P. Argemone P.

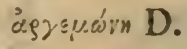
Aloë P. Alopecuris. àrorízesos T. Aruncus. Alsine P. ùr.ín D: Asarum P. Asclepias P. Ascyrum. Aristolochia P. äsıcroxiáa D. Althera $\mathrm{P}$.

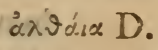
Alysswm P.

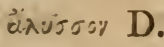
Amrantus.

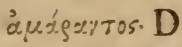
Asporrigus.

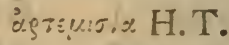
Antrosiz P. áuépssia D. Aspholiclus P. despófer.os D. Anwi. ¿: $\mu u$, D. Asplenium $\mathrm{P}$. ё э H.T.D. zisoryos A. cisaspir D.

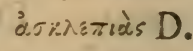

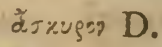

Amomum. cincuv..9 H.T.D. Astar P.

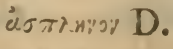

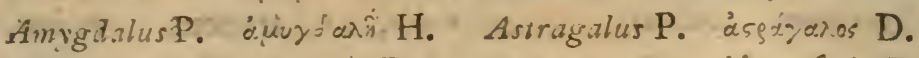

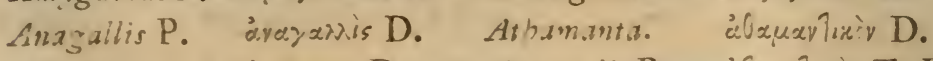
Anagyris P. daytzugis D. Atractylis P. à7saituris T. D.

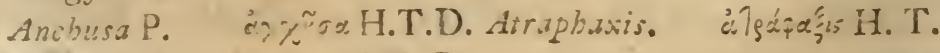
Anethum P. ¿isn जo H.T. D. 
Buccharis P. Fárzapis D. Cerinthe. xhervos T. Ballote P. Eaxistì D. Cestrum. x'śsv D. Borassus. Rógersos D. Chaerophyllum. zarséquzroy O.

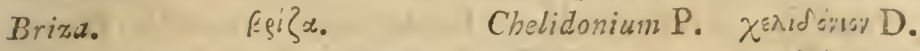

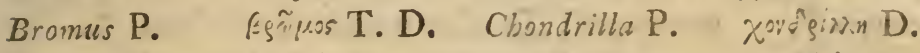

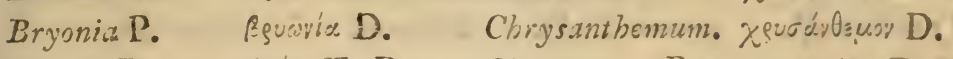

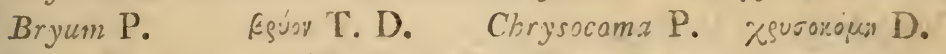
Bubon. Eegóyoy H. Chrysogonum. Xsuróysvoy D.

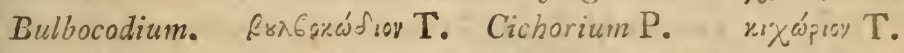

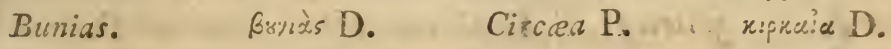

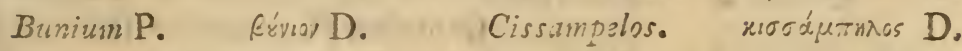

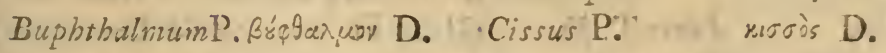
Bupleurum P. kźsisupo: H. Cistus(Cisthus)P.xísos D. Butomus. Rritopss T. Citrus. ritpos D. Byssus P. Eúras Pollo Clematis P. arinuzis D.

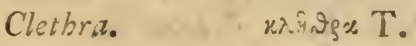

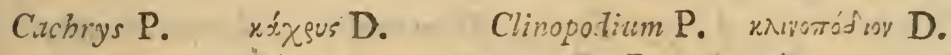
Cactus P. xáitos T. Cneorun P. xyłasoy H. T. Canna O. xávyz Arist. Cnicus P. kvíros H.T. D. Cannabis P. xdirvals D. Crix. roiş T.

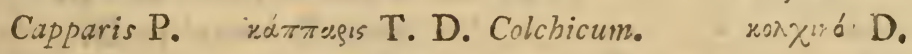

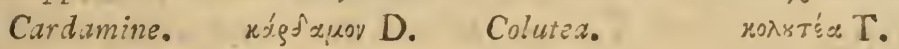

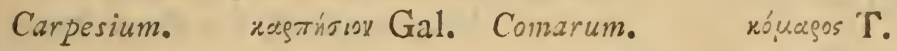
Carum. xósos D. Conium. kúveroy T. D. Caryopbyllus P. kaguóquasior FE. Conyza P. xívu\}a H. D. Caryota P. xusjutàs D. Corcharus P. xígxagos T. C.assia. karoia D. Coriandrum P. kogiaivoy T. D. Catanancle P. katavázkn D. Coris P. xógis D. Caucalis P. navearis H.T. Corymbium. xas $\mu .10 \%$. Ceanotbus. xé́vesosos T. Costus P. - xóros T. D. Cenchrus. xézyess T. D. Cotinus P. xótivos T. Centaurea P. kev7augany H. D. Cotyledon P. kofudescìy D. Cerasus. xegasia T. D. Crambe P.

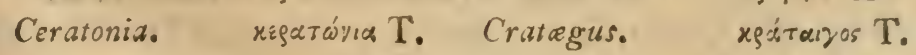
Cercis. xegris T. Crinum $\mathrm{P}$. kęivoy $\mathrm{D}$. 


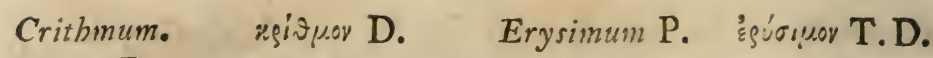

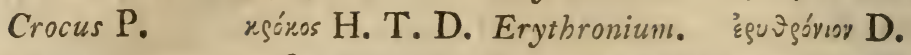

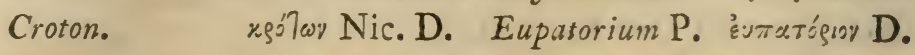

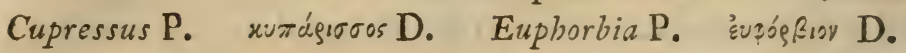
Cyclamen P. жuxлd́puros T.D. Evonymus. Ėvényıs T.

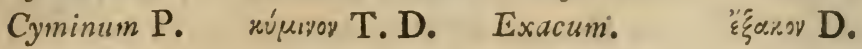
Cynanchum. «نváz $\chi^{n}$.

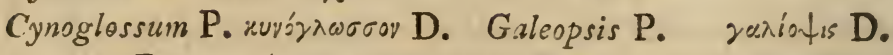

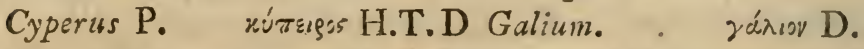

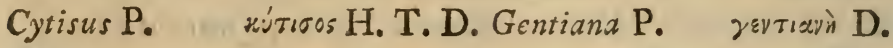
- Geraniam P. yesávisy D.

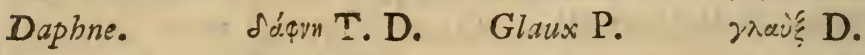
Daucus P. Sajres D. Glecboma. $2 \lambda \dot{x} \chi \omega \gamma$ D.

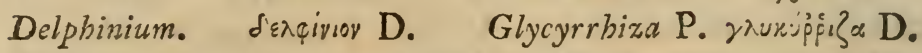
Dictamnus P. dikraupos T.D. Gnaphalium P. zrapid八ıov D. Dipsacus P. Sitarios' $\mathrm{D}$. Dolichos. Sicaryos T. Hamameliso dupulunis Ath.

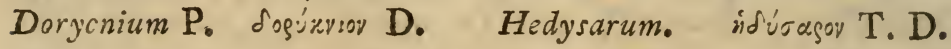

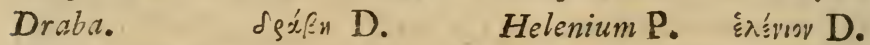

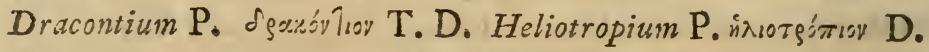

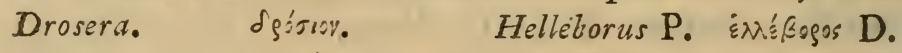

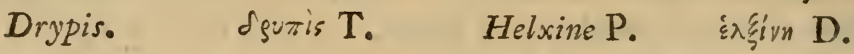

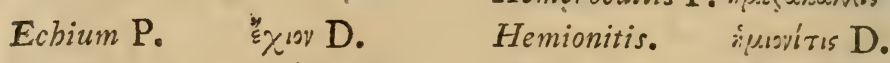
Elaxgnus. Ėaiagros T. Hibiscus. ifíros D. Elatine P. Ėatian D. Hieracium. iegáxiov D.

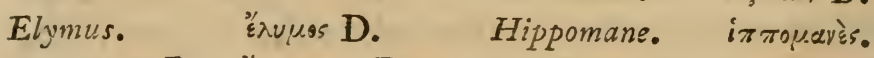

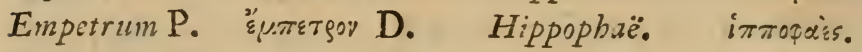

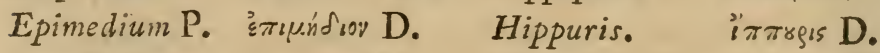

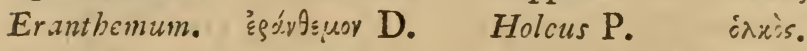

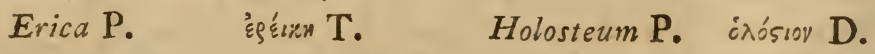

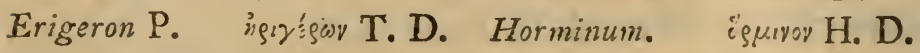
Erinus. $\quad$ ¿sivos D. Hyacintbus P. idxivjos T. D. Eriophorum P. Ésíqogov T. Hydnum. isva D.

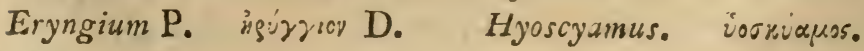


Osyris P. öougis D.

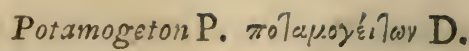

Othonna P. iśrya D.

Poterium P.

Totnifise D.

Oxalis $\mathrm{P}$.

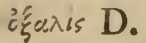

Pothos P.

$\pi$ ivos $\mathrm{T}$.

Prasium.

Tráaiov D.

Peonia P.

malovia H. D. Psidium.

moidras $\mathrm{H}$.

Panaces P. $\quad \pi \alpha^{\alpha} y \alpha_{\xi}^{\xi}$ T. D.

Ptelea.

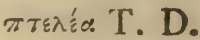

Pancratium P. rayiegx́liov D. Pteris P.

Trípis T. D.

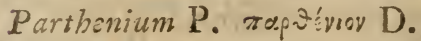

Peganum. : aízavov T. D.

Peplis.

$\pi \varepsilon \pi \lambda i \bar{s}^{\circ} \mathrm{D}$.

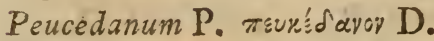

Phaca.

Q̣ax's D.

Phalaris $\mathrm{P}$.

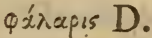

Phaseolus. Corionos T. D.

Rhamnus' $\mathrm{P}$

pépunos T. D.

Rhapbanus $\mathrm{P}$.

papyils 'T:D.

ṕźcaros T.

Rbeum.

piñor D.

Rbododendrum. poofó Evofo\% D.

Rbus $\mathrm{P}$.

คัชัs D.

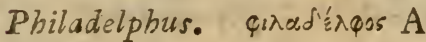

Phillyre.

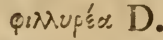

Philyca.

Girúrex $T$.

Pbleum.

Q. ร́, T.

Pblowis.

exćpos D.

Phlox P:

बri६̧.T.

Phonix.

Qoiviśs T. D.

Pbyteuma P.

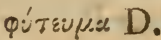

Picris.

тикрѝs D.

Pimpinella.

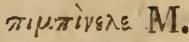

Samyda.

onuivía $T$.

Satyrium $\mathrm{P}$.

oazúprov D.

Scandix P.

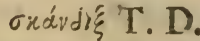

Schinis.

oxivos Athen.

Scbanus.

oxoívos $\mathrm{D}$.

Scilla P.

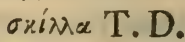

Piper.

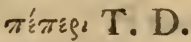

Scolymus P.

oxórupus $\mathrm{D}$.

Selinum.

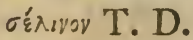

Pistacia.

miśxud T. D.

Sesamus P.

ó́sapov T. D.

Pisum.

mirov T.

Seseli P.

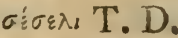

Sicyos.

owuòs $T$.

oidn T.

Platanus $\mathrm{P}$

$\pi \lambda \dot{x} \tau a y o s$ T.D

Silla.

Gidnpitis D.

Poa.

$\pi: \alpha \mathrm{T}$.

Sideritis P.

cir.eior D.

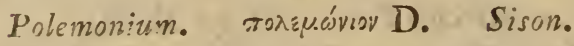

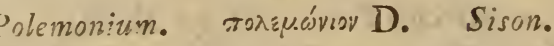

Sinapi P.

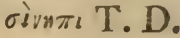

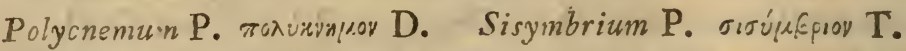

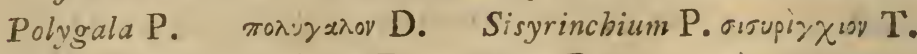

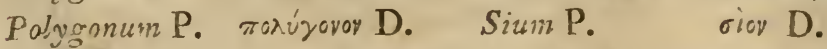

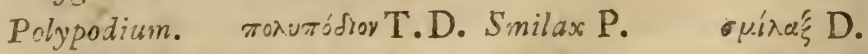

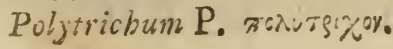

Smyrnium.

ouifirioi D. 
Sonchus P. oóryos T. D. Teucrium P. Ttisper D.

Sparganium.

Spartium.

Sphagnsim P.

Splachnum.

Spongia P.

Stachy: P.

Statice P.

Stabe P.

Stratiotes.

Strutbion P.

Stryctínon P.

Styrax.

Symphytum P.

Taxus, P.

Telepbium P. Tetragonia. orapyóviov D. Thalictrum P. oráp Trov T. D. Thapsia P. opayvir H. D. Thlaspi P.

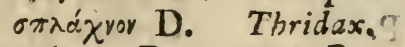
stóvzyos D. Thya P. śxxus D. Thymus. sथराKमे 50 हों D.

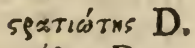
spévioy D. spúxyos D. súcás T. D. oúcaputar D. $\tau$ Táşos Aët.

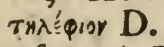
тEใparavia. $\mathrm{T}$.

\section{Vella Gal.}

Tordylium P.

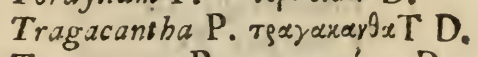
Trigopogon P. tparsászar D.

Tribulus. Tfisodos 0.

Trichomunes. zpixiexvès D. Trifolium. triqunsor H. D. Typhe P. rúp. T. D. Topfódisy D.
Xantbium. $X$ ylon P. Xyris $\mathrm{P}$. $Z_{e a} \mathrm{P}$. Zizania.

vèrix pov D. vartia D.

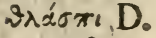
Эpis $\alpha$ \& D. จ.je T. जúpes T. D. .

(


Carex $\mathbf{V}$. Carica P.

Carpinus.

Celtis P.

Centunculus $\mathrm{P}_{\text {: }}$

Cepa P.

Ceraisus P.

Chamerops $\mathrm{P}$.

Chenopus P.

Chironia $\mathrm{P}$.

Cicer p.

Cicutal P.

Cinara $\mathrm{P}$.

Cleome Hor.

Coccus $\mathrm{P}$.

Conferva $\mathrm{P}$.

Convolvulus $\mathrm{P}$.

Cornus P.

Corylus P.

Crepis P.

Cucubalum $\mathrm{P}$.

Cucumis $\mathrm{P}$.

Cucurbita P.

Cunila P.

Cupressus $\mathrm{P}$.

Cycas P.

Dactylos P.

Diospyros $\mathrm{P}$.

Dodecatheos P.

Ephedra P.

Equisetum P.

Erigeron P.

Ervum P.

Esculus P.

Exacum P.

Fala P.

Fagus P.

Ferula P.

Ficus P.

Filix P.

Faniculum P.
- Fragaria P.

Fraxinus $\mathrm{P}$.

Fucus P.

Fungus P.

Genista P.?

Geum P.

Gladiolus P.

Gnidium P.

Gossypium P.

Gramen P.

Hedera P.

Helianthus.

Heracleum.

Hesperis P.

Hordeum P.

Hynsiris $\mathrm{P}$.

Ilex $\mathrm{P}$. $\mathrm{V}$.

Illecebra.P.

Inula $\mathrm{P}$.

Isoëtes $\mathrm{P}$.

Juglans P.

Juncus P.

Funiperus P.

Lactuca P.

Lamiun P.

Laserpitium $\mathrm{P}$.

Laurus P.

Leontice P.

Ligustrum $\mathrm{P}$.

Lilium $\mathrm{P}$.

Lolium P. V.

Lupinus P.

Malva $\mathrm{P}$.

Marrubium P.

Melitis $\mathrm{P}$.

Menyanthes.

Mercurialis $\mathbf{P}$.

Milium $\mathrm{P}$.

Nimmulus $\mathrm{P}$.

Mollugo $\mathrm{P}$.

Mucor Col. 
Nepeta $\dot{\mathrm{P}}_{\text {. }}$ Olea P.

Onopordum $\mathrm{P}$. Opbrys P. Palma P. Panicum P. Papaver P. Pentapeies P. Pezica P. Pharnaceum P.

Pbellandrium.

Pbyllantbes P.

Pinus P.

Pirus P.

Populus P.

Porrum P.

Portulaca P.

Proserpinaca P.

Prunus P.

Punica $\mathrm{P}$.

Quercus P.

Ranunculus $\mathrm{P}$.

Reseda P.

Rbexia P.

Ricinus $\mathrm{P}$.

Rosa P. .

Rosmarinus.

Rubiá P.

Rubus P.

Rumex $\mathrm{P}$.

Ruscus V.

Ruta P.

Saccharuin P.

Salix P.

Salvia P.

Sambucus P.

Samolus P.

Sanguinaria $\mathrm{P}$.
Satureja P.

Saxifragum $\mathrm{P}$.

Scirpus P.

Scorpiurus $P$.

Secale P. I en

Sedum P.

Selago P.

Sempervivum P.

Senecio P.

Serratula P.

Solanum P.

Sorbus P.

Spireon P.

Syringia P.

Tamarix P.

Tamius P.

Thelygonum P.'

Thesium $\mathrm{P}$.

Tilia P.

Tinus P.

Triticum.

Ulex P.

Ulmus $\mathrm{V}$.

Ulva V.O.

Urtica P.

Vacrinium P. V.

Valerianx.

Veratrum P.

Verbascums, $\mathrm{T}$.

Verbena $\mathrm{T}$.

Viburnum V.

Vicia P.

Vinca (per vinca) $\mathrm{P}$.

Viobx $\mathrm{P}$.

Viscus $\mathrm{P}$.

Vites.

Vitis.

Zostera P. 
342. Nomen genericum antiquum (24r) antiquo generi convenit.

Metamorphosis Lingue Latinæe in Græcam:

Dens Leonis

Ferrum Equinum

Nidus Leporis

Transimutatio sensualis:

Acacia Robini

Gramen Parnassi

Lilium convallium

Jan-Raja

Abreviatio.

I. Achyracantha

2. Calophyllodendron Staphylodendrum

Tetragonocarpus

Leontopetalon

Heleniastrum

Partheniastrum

Arachidna

Sicyoides

3. Oreoselinum

Melocactus

Anapodophyllum

Hydroceratophyllum
Leontodon.

Hippocrepis.

Lagacia.

Robinia.

Parnassia.

Convallaria.

Rajania..

Achyrantbes.

Calophyllum.

Staphylaa.

Tetragonia."

Leontice.

Helenia.

Parthenium.

Arachis.

Sicyos.

Selinum.

Cactus.

Podophyllum.

Ceratophyllum.

4. Ananthocyclus

Anacyclus.

Asteris genus antea comprehendit sub se Enulam campa-

nam, et affines; at detecto charactere essentiali, nequit

tritissima planta assumere novum nomen, hinc antiquissimum Inulse receptum.

243. Nomen genericum dignum $\left(213^{-242}\right)$ alio, licèt aptiore, permutare non licet.

Asclepias floribus Muscas captat, hinc vocabulum Myagrum aprissimè huic generi competeret.

Menyanthes ob florem lanatum a ptiùs Erianthus, aut Lasianthus dici posset essentiali nomine.

Sed abstinendum ab hac innovatione, quæ nunquam ces-

saret, quin in dies aptiora detegerentur in infinitum. 244. Nomina Generica, quamdiu Synonyma digna in promtu sunt, nova non effingenda. 
Noris e. c. detectis Generibus nova nomina aptissimè conficiuntur, et iisdem imponuntur.

Antiquum si dirimatur genus in plura, consultum est nova non effingere nomina generica, quamdiu digna in phalange synonymorum specierum supersint.

245. Nomen genericum unius generis, nisi supervacaneum $(2 \mathrm{I} 5-2 \mathrm{I})$ ), in aliud transferri non debet, licèt eidem aptiùs competeret.

Quis longo ævo recepta vocabula commutaret hodie cum Patrum?

Hyacinthus veterum est Dolpbinium.

Tribulus ......... Fugonia.

Opulus ......... Humulus:

Botanici sæeculo XVI. inquirentes veterum vocabula, Botanicen ferè perdiderant.

Notissima sint genera A. B. C. D. secundìm nostra genera plantarum.
A. Lithospermum.
B. Myosotis.
C. Alsine.
D. Cerastium.

Tournefortius genus B sub A comprehendit, adeoque nomen $\mathrm{B}$ in $\mathrm{D}$ transtulit.

Ruppius distinxit genera $\mathrm{A}$, et $\mathrm{B}$, et nomen Myosotic $\mathrm{T}$. revocavit a $D$ ad $B$, et gęnus $D$ sub $C$ reduxit.

Dillenius genus $C a b D$ distinxit cum Tournefortio, nec D uniit cum C, uti Ruppius; Genera etiam A , et B distincta cum Ruppio servabat, nec ut Tournefortius hæc A B miscuit; adeoque nomen Myosotis plantæ B concessit, novum autem (Cerastium) D imposuit. Tyro qui antea conjunxit vocabulum Myosotis cum genere $\mathrm{D}$ ex sententiâ Tournefortii, nunc verò tenetur idem combinare cum $B$ secundùm Ruppium, hæesitat semper, utrum hoc cum $B$ aut $D$ conjungat.

246. Si Genus receptum secundùm jus naturæ ( 162 ), et artis (164), in plura dirimi debet; tum nomen antea commune manebit vulgatissimæ, et officinali plantæ.

Corni genus supponatur dividi in tria:

A. Arbor floribus involucratis, umbellatis. 
B. Herba floribus involucratis, umbellatis.

C. Arbor floribus non involucratis, cymosis.

Sic dicenda A. Cornus, B. Mesomora, C. Ossex, nec licet A dici Mesomoram, aut Osseam.

247. Nomina generica (229) latinis literis pingenda sunt.

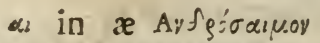

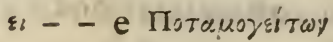

- - i A's! $3 \omega \%$

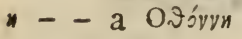

- - e Ninevinis

- - o Opokos

- - u $\Upsilon$ r ŕrooy

ov - - U A $A^{a} \gamma$ xouga

or - oe $\Phi$ Jivis

$\omega$ - o Sícoy

₹ - th Boi $q v \alpha \lambda \mu \nu \gamma$

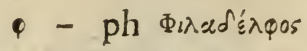

x - ch Xaiǵ́quiroy

$x$ - $\mathrm{c} \Omega\left(x \mu \mu_{0} \%\right.$

$\gamma \chi-n c h A^{\prime \prime} \gamma x$ ous\%

$\gamma \gamma$ - ng Hérzroy

- h Avrípalyoy
Androsemum.

Potamogeton.

Aizoon.

Otbonna.

Nepentbes.

Orobus.

Hypecoum finale.

Ancbusa.

Pboenix.

Sison.

Bupbtbalmum.

Pbiladelpbus.

Cberopbyllum.

Ocimum.

Ancbusa.

Eryngium.

Antirrbinum.

248. Terminatio, et sonus nominum genericorum, quantum fieri possit, facilitanda sunt.

Insuetæ terminationes. in e Ballote.

- i Sinapi.

Seseli.

Thlaspi.

- ois Hedypnois.

- $t$ Tetrabit.

- n Triglochin,
FEquivoca. Alpina P1. Alpinia.

Pbyllum Phyllis.

Meum $\mathrm{T}$. Athamanta.

Perversa.

Antboceros M. Ceranthus. Caraxeron V.

249. Nomina generica sesquipedalia, enuntiatu difficilia, vel nauseabunda, fugienda sunt. 


\section{- . - - - Sesquipedalia verba}

Vel nocitura sono, guttur leesura loquentis

Sesquipedalia mihi quæ plures, quàm duodecim literas admisere:

17. Kalopbyllodentron V. Calophyllum.

18. Titanoceratophyton $\mathrm{B}$. Isis.

19. Leuconarcissolirion

Galanthus.

21. Coriotragematodendros Plk. Myrica.

22. Hyp phyllocarpodeniron B. Protea.

Difficilia ex pluribus consonantibus conjunctis.
Acrochordodendros $\mathrm{Pl}$.
Stachyarpogophora V.
Orbitochartus Kn.
Cephalanthus.
Achyranth:s.
Fagonia.

Niuseosa, quæ nescio quod insueti produnt.
Caraxeron V.
Galeobdolon D.
Gomphrena.
Myrobatindum V.
Leontrus.
Morinda.

250. TERMINIs artis (I99), loco nominum genericorum, abuti, inconsultum est.

Latina.

Tuberosa $\mathrm{H}$.

Graminifolia R.

Spica Hk.

Siliqua T.

Nus $\mathrm{T}$.

Odorata R.
Græca

Polyanthes. Polyanthes Pt.

Subularia. Pbyllon.

Lavandula. Hexapetala Pk.

Ceratonia.

25I. Nominum Classium ( 160 ), et Ordinum ( 16 I), cum genericis par est ratio (204).

Leges Generis datas subeunt nomina Classium

Propria: \$.213. 214.217.

Verbo unico: \$. 215.221.

eodem: 216.

non primitivo: 220.

non bybrids: $223 \cdot 224 \cdot 225 \cdot 226.227$.

non barbaro: 229.

non requivocs: $230.23 \mathrm{r}$.

non contrario: 232.

non a viris: 2,3 .

non sesquipedulia: 249 .

non difficilia: 248 . 
Casalpinus definitiones loco nominum classium usurpavit. Tournefortius, Rivinus, ct antecessores nonnulla vocabula srepius admittebant.

252. Nomina Classium, et Ordinum e Viribus, $R a-$ dice, Herbâ, et Habitu petita, mala sunt.

Systematica vocabula debent esse essentialia ex fructificatione desumta.

Fragmenta methodi Naturalis ab accidentalibus nomina desumsere, utpote vicaria, cùm absoluto systemate, necesse est, ut mutentur omnia ex lege futuri divisionis systematis.

Plantis dubii generis imposui semper erronea nomina, in Oides, vel aliter diminutiva: cùm planta debuit in Catalogo numerari, neque adhuc constaret de fructificatione; adeoque impossibile erat etiamnum verum nomen; sic lectores a solo erroneo vocabulo moniti, me de genere incerto, inquirerent attertiùs in fructificationem.

a Viribus. Radice. Foliis. Habitu.
Cordialis
Bulbose Asperifolice
Capillaris
Tuberose
Fibrosa
Succulentre
Verticillate
Arbores
Frutices

253. Nomina Classium, et Ordinum notam essentialem (187), et Characteristicam (189) includant.

Ratio : cùm Methodi plurimæ, mutabiles, modernæ. cùm nomina sint onera artis inevitabilia. cùm parata, et promta omni momento erint nomina. Rectè: Bene: Malè.
Calyciflore.
Siliquasa.
Papilionacere $\mathrm{T}$.
Cruciformes $\mathrm{T}$.
Leguminose.
Discoidece. 1
Difformes.
Syngeniste.

254. Nomina Classium, et Ordinum, a plantee cujusdam nomine desumta, sub quo totam cohortem intellexere veteres, in genere exclusa, classibus naturalibus tantùm inserenda. 


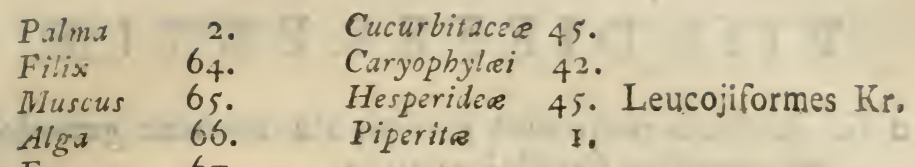

Fungus 67.

Gramen I 4 .

Lilium - 10. Lirium Roy.

Rhocas 30.

Corydalis 28.

Calamus 13.

Orchis

4.

Inserenda Classibus, et Ordinibus naturalibus tantùm sunt ejusmodi plantarum vocabula, aliàs nunquam; alioquin, ut nov $x$ Methodi non cessant, ita nec cessarent Botanici plantarum nomina generica excludere, classibusque artificialibus inserere; hoc autem minùs metuendum in $\mathrm{Na}$ turali, quæ unica est, et erit.

255. Nomina Classium, et Ordinum unico (2 I 5) vocabulo constabunt.

Radiati T. Compositi ex irregularibus in ambitw, et regularibus in medio Riv.

Flore perfecto simplici, seminibus nudis solitariis, seu ad singulos flores singulis Raj.

Pulchra ex uno vocabulo.
Campaniformes
Monopetali
Monandria
Infundibuliformes
Personati
Dipetali
Tripetali
Labiati
Tetrapetali
Diandria
Cruciformes
Pentapetali \&ic. Pentandria \&ce.
Rosacei \&c.
Triandria
Tetrandria

- Cavendum, ne nimis longa, nimisque composita evadant hrec vocabula. 


\section{I I I. D I F F E R E N T I Æ.}

256. Perfectè nominata est planta nomine generico. et specifico (2 I2) instructa.

Botanices Tyro novit Classes, Candidatus omnia Genera, $M a$ gister plurimas species.

Q:io plures Botanicus noverit species, è etiam prestantior est. Cognitione specierum innititur omnis solida eruditio Physica,

Osconomica, Medica; immo omnis vera cognitio humana. Speciei notitia consistit in notâ essentiali, qua sola ab omnibus congeneribus distinguitur.

Sine notitiâ Generis nulla certitudo speciei.

Casalpinus: Ignorato genere nulla descriptio, quamvis adcuratè tradita, certam demonstrat, sed plerumque fallit. Differentia specifica continet notas, quibus species a congeneribus differt.

Nomen specificuin autem continet Differentix notas essen tiales. Notæ in no nine specifico sint

non lubrica, incertic, aut false $\$ .259-274.29 \mathrm{r} .283$.

sed firme, cerice, miectidnicre $\oint$. 275-290. 257.287.

quxe cauté, castè, judiciosi \$. 284-305.

257. Nomen specificum legitimum plantam ab omnibus congeneribus (159) distinguat; Triviale autem nomen legibus etiamnum caret.

Fundamentum est hic canon nominum specificorum, quo neglecto, lubrica erunt omnia.

Nomina specifica omnia, quæ plantam a congeneribus non distinguunt, falsa sunt.

Nomina specifica omnia, qux plantam ab aliis, quàm congeneribus distinguunt, falsa sunt.

Nomen specificum est itaque Differentia essentialis.

NO VINA TRIVIALIA fortè admitti possunt modo, quo in Pane suecico usus sum; constarent hrec

Vocabulo unico;

Vocabulo liberé undequaque desumto.

Ratione hac præcipuè evicti, quòd differentia sxpe longa evadit, ut non ubique commodè usurpetur, et dein mutationi obnoxia, novis detectis speciebus, est. e. gr. 
Pyrola irregularis.

Pyrola Halleriana.

Pyrola secunda.

Pyrola uinbellata.

Pyrola unif:ord.
Pyrola staminibus adscendentibus, pistillo declinato. Fl. suec. 330 .

Pyrola floribus racemosis dispersis, staminibus pistillisque rectis. $\mathrm{Fl}$. suec. $33 \mathrm{I}$.

Pyrola racemis unilateralibus. Fl. suec. 332 .

Pyrola floribus umbellatis.Fl. suec. 333 .

Pyrola scapo unifloro. Fl. suec. 334 .

Sed nomina Trivialia in hoc opere seponimus, de differentiâ unicè solliciti.

258. Nomen specificum primo intuitu plantam suam manifestabit, cùm differentiam (257) ipsi plantce inscriptam contineat.

Trivialia erant antecessorum, et maximè Trivialia erant antiquissimorum Botanicorum nomina.

Character Naturalis speciei est Descriptio; Character verò Essentialis speciei est Differentia.

Primus incepi Nomina specifica Essentialia condere, ante me nulla dif̃erentia digna extitit.

Agnovere hanc Methodum Lotanici recentic res acutissimi Koyenus, Gronovius, Guettardus, Dalibarilus in omnibus, Hallerus, Gmelinus, Burmannus in plerisque.

Mea nomina specifica e'Descriptione extraxere Differentias; ex Differentiis selectissimum investigarunt characterem essentialem, quo constant.

Exciudendæx itaque sunt, in nomine specifico, notæ omnes accidentales, in plantâ ipsâ non exsistentes, aut ron palpabiles, ex gr. Locus, Tempus, Duratio, Usus.

Erronea Nomina Specifica sunt omnia ab idearum ordine, aut hypothesi desumta.
Ordines ilearum.
Tinus prior.
- - alter.
- tertius.
Hypothesis.
Hyoscyamus peculiaris.
Meum spurium.
Acorus verus.
Campanula pulchra. 
259. Nomen specificum a partibus plantarum non variantibus desumi debet.

$V$ arietates esse distinctas species, nullus sanus facilè dixerit in Regno Animali;

Vaccas albas, nigras, rubras, cinereas, variegatas. Vaccas parvas, et magnas, macras, et pingues, laves, et pilosas, nullus dixit totidem diversas esse species.

Canes meliteos, fricatrices, mastivos, grajos, lanatos, \&c. esse ejusdem speciei,docuere Verrucæ, Vortices,Suturæ. Species ex varietatibus apud antecessores multiplicarunt

Metus confundendi diversas;

Defectus Differentiarum essentialium;

Ignorantia continuatæ generationis specierum; $\$ .79$. I 32. Cognitio obscura speciei;

Anthophilorum rabies contagiosa;

Subtilitatis studium \&c.

Variantes maximè sunt, et rarò constantes, Color, OJor, Sapor, Hirsuties, Crispatio, Impletio, Monstrositas.

Patroni varietatum, qui varietates in specierum numerum adoptarunt, fuere ante nos præcipuè recentissimi: Barrelier, Tournefortius, Boerhaxvius, Pontedera, Michelius.

Conspurcavit magis Botanicem varietatum introductio, quàm alia res ulla; confundebat hæc ita synonymistas, ut nisi brevi medela, actum fuisset de scientia.

Erronece differentice sunt, quæ varietates loco speciei tradunt. TRIFOLIUM capitulis subrotundis, flosculis pedunculatis, leguminibus tetraspermis, caule procumbente. Hort. cliff. 375 .

Trifolium pratense album. Bauh. pin. 327.

Trifoliastrum pratense corymbiferum majus repens, foliis rotundioribus, albâ, et sagittatâ maculâ notatis, siliquis tetraspermis. Mich. gen. 26.

2. Trifoliastrum pratense corymbiferum majus repens, foliis rotundioribus, albâ, et sagitiatâ maculâ notatis, corymbis florum magis sparsis, pediculis longissimis insidentibus, siliquis tetraspermis. Mich. gen. 26. t. 25. f. I.

3. Trifoliastrum pratense corymbiferum majus repens, foliis rotundioribus, alb $\hat{a}$, sagittat $\hat{a}$, et in acutum longius product $\hat{a}$ maculâ notatis, siliquis tetraspermis. Mich. gen. 26 . t. 25 . f. 4 . 
4. Trifoliastrum pratense corymbiferum majus repens, foliis oblongis obtusioribus, maculâ atbicunte supernâ parte pyramidatâ, infernâ cordis instar pulcbrè excavatâ, siliquis tetraspermis. Mich. gen. 26 .

5. Trifoliastrum pratense corymbiferum majus repens, folio subrotundo, albâ, semilunat $\hat{a}$, et in dorso nonnibil excavat $\hat{a}$ maculâ notato, siliquis tetraspermis. Mich. gen. 26 .

6. Trifoliastrum pratense corymbiferum majus repens, folio corlato, albầ maculâ ejusdem forme insignito, siliquâ tetraspermá. Mich. gen. 26.

7. Trifoliastrum pratense corymbiferum majus repens, folio obtuso duabus maculis albis insignito, quarum superior minor triangularis, inferior major cordata, siliquâ tetraspermá. Mich. gen. 27.

8. Trifoliastrum pratense corymbiferum majus repens, foliis obtusis, et veluti cardatis, non maculatis, siliquis tetraspermis. Mich. gen. 27.

9. Trifoliastrum pratense corymbiferum repens medium, foliis oblongis acutioribus, maculâ latâ, sagittatâ, siliquis tetraspermis. Mich. gen. 27.

10. Trifoliastrum pratense corymbiferum repens medium rotun-

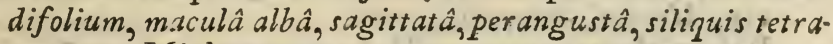
spermis. Mich. gen. 27.

11. Trifoliastrum pratense corymbiferum repens minus, folio subrotundo, maculâ albâ, sagitiatâ, minimâ. Mich. gen.27.

12. Trifoliastrum pratense corymbiferum repens minimum, foliis obtusis non maculatis, siliquis tetraspermis, supernâ parte equalibus, infernâ veluti nodosis, semine subluteo. Mich. gen. 27. t. 25. f. 3 .

13. Trifoliastrum annuum corymbiferum album, et procumbens, folio cordato subtus atro-virente splendente, siliquâ tetrasper. mâ, infernè falcation discriminatâ. Mich.gen.27.t.25.f.6.

14. Trifoliast rum pratense corymbiferumnon repens, humi stratum, altè radicatum, foliis subrotundis, albâ, falcatâ maculâ vix notatis, floribus minoribus suave rubentibus, siliquis tetraspermis supernâ parte tantum marginatis, semine fusco. Mich. gen, 27. 
15. Trifoliastrum pratense corymbiferum erectum annuum, et praaltum, caule crassiore, et fistuloso, folio longiore cordiformi, flore albo, siliquâ incurvâ, lat $\hat{a}$, compressa, ac dispermâ. Mich. gen. 28.t. 25. f. 2 .

16. Trifoliastrum supinum corymbiferum annuum album majus, folio longiori obtuso, siliquâ incurvâ, lat $\hat{a}$, compressâ, ac dispermâ. Mich. gen. 28. t. 25. f. 5.

Exemplo horrendo sapiant Botanici, ne minima circumstantia variet rem contra legem Naturæ, cui specierum generationem commisit Creator, non hominibus; impegit enim Michelius oculatissimus contra $\$ .262$ : Foliis rotundioribus, longioribus, acutioribus, obtusioribus: Caule crassiore: Pedunculis longissimis \$. 260 : Caule pralto \$. 266. Maculâ foliorum colore, et figurâ diversầ: Floribus suave rubentibus: Seminibus fuscis, et subluteis.

260. Magnitudo species non distinguit.

Magnitudo mutatur a Loco, Solo, Climate; mutatur a copiâ alimenti in plantis, non minùs, quàm in animalibus.

Magnitudo, si varians sit, nec mutans speciem, nequit differentiam essentialem nomini specifico subministrare.

Erronea Nom. Spec. sunt omnia a magnitudine Plante, Radicis, Herba, Fructificationis.

Polytrichüm maximum Pluk. minus est, quàm Thalictrum minimum.

Plintagò major, a nonnullis media cognominata. J. B. a Plantâ.

Alsine altissima

a Foliis.

- - major

Nicotiana augustifolia

- . media

latifolia.

- minor

- - minima

- exigua.

Sedum majus

- - minus

- - parvum

- - minimum

Boletus magnus.

Galeopsis procerior.

Gramen elatius. 
Fraxinus excelsior.

Trachelium giganteum.

Jasminum bumile.

Virga aurea bumilior.

Salix pumila.

Betula nana.

Melampyrum perpusillum.

261. Notæ collatitice cum aliis speciebus diversi generis falsæ sunt.

Præsupposuerunt antecessores apud Tyronem empiricam cognitionem plerarumque plantarum Europæarum, tanquam ex ides innatis, adeoque in arte Doct is scripsere; nos autem indoctos docere tantùm studemus.

Lege artis mutuò noscatur planta ex ncmine, et nomen ex plantâ; utrum que ex proprio charactere; in illo scripto, in hac delineato; Tertius non admittatur.

Nomina præsupponentia alias plantas deduxere tandem homines in vertiginem.

Erronea Nom. Specif. proclamamus ea, quæ similitudinem alterius plantæ ex Herba, Fructificatione, Habitu, grecè aut latinè gerunt.

a Foliis.

Jacobæa

Betonice folio.

Glasti folio.

Chrysanthemi folio.

Rosmarini folio. Absinthii folio. Hieracii folio. Hormini folio. Sonchi folio.

Dentis leanis folio,

Helenii folio.

Limonii folio.

Senecionis folio:

ab Imaginatiore. per Vertiginem.

Jacobra Hicrucii folio.

Hieracium Blattarice folio.

Blattaria Verbasci folio.

Verbascum Conyze folio.

Conyza Salvice folio.

Salvia Hormini folio.

Horminum Betonice folio.

Betonica Scrophularice folio.

Scrophularia Melisse folio.

Melissa Plantaginis folio.

Plantago Coronopi folio.

Coronopus Senecionis folio. Senecio facobre folio.

Clinopodium Origani facie. Adonis Buphthalmi flore.

Ocymi facie. Acer Platanoides.

Brassica Asparagoides. 


\section{Cirsium Helleborinigr. rad. Carduus Centauroides. Adonis Helleboroides. Vicia Lathyroides. Pseudo-HelleborusRanunculoid.}

262. Notæ collatitice cum aliis speciebus ejusdem generis malæ sunt.

Nomen specificum, nisi præsentibus omnibus congeneribus, solidè non fabricatur, cùm contineat notam, in congenerum nullâ exsistentem;

Magistri est condere nomen, Tyronis ex eo nosse plantam. Tyro nequit colligere species, sed nosse debet unam post alteram, cùm simul nec nascantur, nec exsistant individui comites.

-Erronea Nom.Specif. sunt, quæ præsupponunt alteram speciem notam.

Orchis flore candidissimo $\mathrm{T}$.

Campanula angustifolia, magno flore, minor $\mathrm{T}$. Campanula, flore minore, ramosior Moris.

263. Inventoris, vel aliùs cujuscumque nomen in Differentiâ non adhibeatur.

Plantarum manus sunt nomina, Dextra Genericum, Sinistra Specificum; manus plantarum oculatæ sunt, credunt ea solùm, quie vident; has porrigat Botanico Planta, si fides habenda rebus.

Notâ sic plantâ promit Scientia omnia occulta : Historica, Synonyma, reliqua.

Erronea Nom. Specif. a viris dicta esse omnia statuimus, sive ea ab Inventore, seu a Descriptore, seu ab Historia, sive in Memoriam efficta sunt.
ab Inventore.
ab Historiâ.

Trifolium gastonium Moris. Sideritis Valerandi Dourez. I. B. a Descriptore.

Gramen cyperoides $B \propto$ lii Lob.

Campanula a Tossano Carolo Conyza tertia Dioscoridis C.B. Mimosa a Domino Hermans, exConyza media Matbioli I. B. Narcissus Tradescantii Rudb. cellentissimi D. Syen. Breyn. in Memoriam.

Chamæpithys flore plusquam eleganti. s. Pluqueneti Pluk. Eriocephalus Bruniades Pluk. Amanita Divi Georgii Dill. 
264. Locus natalis species distinctas non tradit.

Locum non debere Nomen Specificum intrare, multæ rationes suadent.

I. Nullus facilè adiret Japoniam, Caput Bonæ Spei, Peruviam, ut nosceret plantam.

2. mutatur sæpius Locus. Alpinæ plantæ omnes, summèque montanæ, extra alpes paludosæ evadunt.

3. non unicus est locus ejusdem speciei ;

Lapponia, Sibiria, Canada,

Asia, America easdem sæpius alit.

4. Hortus Paradisiacus coërcet sæepe ex toto orbe plantas.

5. Quis plantam sibi porrectam absque loco non studeret dignoscere?

6. Botanicis in Herbario, Medicis in Fharmacopolio arridet nosse species.

7. Respectivus est locus ad nos, nostramque cognitionem in Europâ.

Planta sine loco concipi nequit; accidentalis tamen est, licèt proximus; mutabilis vero maximè, hinc nequit nomen specificum intrare.

Erronea Nom. Specific. a Loco desumta statuimus omnia esse, sive ea a Solo, sive a Regione, sive a Frequentiâ desumta sint. a Solo

Valeriana silvestris. a Regione. palustris. Acrostichum campestris. Calceolus Mar.

Europea. montana. Pentaphylloides alpina. Bugula

Mentha arvenis. Acetosa Septentrionalium. Lapponum Rd. Suecicuin Pluk. Suecica Mor.

\begin{tabular}{|c|c|c|c|}
\hline & aquatica. & Cochlearia & D.tnica C. B. \\
\hline Alsine & nemorum. & Cochlearia & Batavica $\mathrm{Hr}$. \\
\hline & pratensis. & Cochlearia & Britannica Dod. \\
\hline Vicia & segetuin. & Cytisus & Germanicus. \\
\hline & sepium. & Pulmonaria & Gallica. \\
\hline & dumetorum. & Tamariscus & $\begin{array}{l}\text { Narbonensis. } \\
\text { Florenting. }\end{array}$ \\
\hline & rupestre. & Salicornia & Cretica. \\
\hline & mans uvida. & Aster & Atticus. \\
\hline Ery & m juxta muro & R Rnunculu & Turcicus. \\
\hline
\end{tabular}



a Frequentia.
Iris
Chalcedonica.
Iris
Damascena.
Clematis peregrina C. B.
Lilium
Stoechas
Persicum.
Scabiosa communior J. B.
Hydrocoiyle vulgaris.
Fritillaria
Virga aurea
Arabica.
Muscus vulgatissimus.
Virga aurea
Filix
Capitis Bone Spei.
Nove Anglia. Marilandica. Erasiliensis.

Oenanthe rara $\mathbf{R}$.

Valeriana bortensis.

265. Tempus fiorendi, vegetandique maximè fallax est differentia.

Tempus est accidentale plantæ, nec in plantâ existens, sed potiùs Planta in tempore; mutationi obnoxia sunt tempora plantarum, nec partes earum constituentes.

Plukenetius cum coætaneis introduxere ex Indiis vastissimam plantarum messem, nec genere, nec specie definitam; nescio itaque, an majori Botanices emolumento, an onere majore. Destruenda est domus fundamento infido inædificata, reædificanda solida; quidquid e veteri inservit, assumendum, rejicienda verò reliqua, licèt opus tardè fastigium attingeret; Sic etiam in nominibus specificis, ut tandem firmo stet talo Scientia.

Erronea Nom. Specif. a Tempore desumta judico, sive ea ab Anno, s. a Mense, s. ab Horâ desumta sunt. ab Anno.

Tulipa procox.

Tulipa serotina.

Crocus vernus.

Geranium estivale.

Crocus autumnalis. Aconitum byemale. a Mense.

Rosa omnium calendaruin.

Viola Martia.

Rosa Majalis.

Boletus Fulii mensis D.

Boletus Augusti Mensis D. ab Horis.

Lychnis Noctiflora.

Althæa Horaria.

266. Color in eadem specie mirè ludit, hinc in differentiâ nil valet.

Coloris inconstantia in animalibus domesticis præclpuè elucet.

Colore in floribus nihil ineonstantius est; Rubri, et Crerulei flores inter omnes facillimè, et sæpissime in Album transeunt. S. 313 . 
Flores Mirabilis, et Diantlji barbati in eadem plantâ diverso colore ferunt corollas.

Color maximè sensum subtilissimum attrahit, et delectat.

Summâ itaque oscitantiá facillimè ad colores revocabant veteres, sed nimiùm ne crede colori.

Anthopbilorum studium inde natum in opprobrium artis, ut mor-

talium nulli longiùs extra oleas vagari videntur ; patet

hoc ex Tulipâ, Pulsatillâ, Ranunculo, Hyacintho, Primulà. Tournefortius castra anthophilorum intrans vidit, tanquam po-

lyedro in uno Hyacintho 63 , et in unicá Tulipá 93 species, quàm re ipsâ essent, plures.

Erronea Nom. Specif. a colore desumta statuimus esse omnia, sive hæe a Flore, s. a Fructu, s. a Seminibus, s. a Radice

s. a Plantâ, s. a Foliis, s. per Imaginationem desumta. Colorata folia dicuntur, cùm alium assumant colorem, quàm viridem.

Hæc variant maximè, et sæpius colorem peregrinum deponunt.

Albo-Maculata: Cyclamen, AcetosaItalica, Ranunculus repens, Trifolium album, Amarantus emarginatus.

Nigro-maculata: Ranunculus Ficaria, hederaceus, Arum, Galeobdolon D. Hypocharis, Persicaria ferrum equinum referens T. Orchides.

Rubro-Maculata: Ranunculus acris, Nymphoides folio maculis purpureis notato T. Amarantus tricolor.

Tessellata: Satyrium. Fl. suec. 732 . Cypripedium Fl. suec. 736.

Punctata subtus: Anagallis, Plantago maritima Fl. suec. 127.

LINEA aLBA: Arundo indica Cornut. Phalaris gramen pictum, et subtus Empetrum.

Limbo Argenteo: llex T. Buxus T. Caprifolium $T$. Glechoma T.

a Flore.

Primula veris

Auricula ursi f. foccineo.

luteo flore.

rubro.

albo.

purpureo.

violaceo.

variegato.

a Planta.

Brassica

- - -

- -

Marrubium

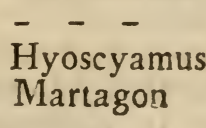

$\mathrm{O}_{2}$ viridis. rubra. alba. album. nigrum.

niger. cruentum. 
a Fructu.

Melo fructu luteo.

Cucumis fructu albo.

Pepo fructu variegato.

Prunus fructu atrocaruleo.

Prunus fructu fiavo.

Prunus fructu cerei coloris.

$$
\text { a Seminibus. }
$$

Papaver semine albo.

Papaver semine nigro.

Sinapi semine rufo.

Sinapi semine lutco.

$$
\text { a Radice. }
$$

Daucus radice atro-rubente. radice aurantii coloris. radice luteâ. a Foliis.

Agrifolium fol. ex luteovarieg. _ - . - fol. ex albo varieg. - . - - fol. limbis, et spinis

Ocymum maculatum. argenteis. aureis.

Esula punctis croceis notata. Malva foliis margine superiùs micis sulphureis ad solem splen. dentibus donata Mloris. ab Imaginatione.

Alypum, s. Frutex terribilis. Campanula pulchra I. B.

Filix scandens perpulchra Br. Poinciana fore pulcherrimo $\mathrm{T}$. Filix saxatilis elegantissima.

267. Odor speciem nunquam clarè distinguit.

Olfactus examinat tenuissima effluvia; obscurus maximè inter sensus; genera paucissima nominat.

$\checkmark$ ariat omnium facillimè odor, et diversus in diversis subjectis.

Quot corpora olida, totidem distinctos esse odores, etiam in eadem specie, patet ex canum indagatione heri in turbâ hominum.

Odores limites determinatos non admittunt, nec definiri possunt; adeoque inter characteres nostros vagos exulandi pro notâ characteristicâ.

Erronea Nom. Specif. meritò proclamamus ea omnia, quæ 1. pro differentia olfactum admittunt.

a Speciebus odoris.

Hy pericum bircinum.

Nielo moschatus.

Agrimonia medio modo odora-

Arbor merdain olens.

$$
\text { ta, M. }
$$

a Tempore.

Hesperis noctu olens.

Caryophyllus inodorus. a Plantis aliis.

Ocymum caryopbyllatum C. B. citri odore. anisi adore. faniculi odore. melissre odore. cinnamomi odore. rute odore.

styracis liquidi odore.

268. Sapor pro ratione manducantis sæpe variabilis est; hinc in differentiâ excludatur. 


\section{DIFFERENTIA.}

Atrates diversæ diversè judicant de sapidis. Solum diversum, et clima mutat sapores.

Cultura edulcorat acida, et acerba.

Cichorium sylvestre amarum.

LActuca silvestris virosa.

Allium in Græciá foetoris expers.

Apium palustre ingratum.

MaLA in sylvis acidissima.

Horreos fructus Pyri, Mali \&cc. tam numero sâ sobole amplificavit ars Pomaria, ut Pyra 172 , Poma 200 distincta produxerit (Teste Boerhaavio), quorum singula solent, proprio nomine condecorari ob singularem saporem.

Erronea Nom. Specif. a sapore ludicra sunt omnia, hine in differentiis omnino excludenda judicamus.

a Plant $\hat{a}$

- Apium ingratius.

dulce.

Lactuca opii succo viroso. mitis. a Fructu.

Pisum cortice eduli.

Pyrus fructu saccharats ore deliquescente.

269. Vis, et Usus Differentiam Botanico vanam subministrant.

Species ut noscatur, debet methodo veterum experimentum fieri in anima vili.

Hippomanem si gustes, videbis experimenta periculosissima. Arum summis labris degustantes mutos reddens. Sloan.

Pharmacopæorum plantæ, et nomina inter synonyma sint: isti non magis nomina præscribant Botanicis, quàm ipsi, ex Botanicis principiis, recedunt a suo usu.

Anne ex uno genere Convolvuli Turbith, Mechoacanna Scammonium \&c. Pharmàcopæeorum gratiâ conficienda, contra leges Creatoris, totidem genera?

Anne ex unâ, eademque Punicâ plura genera plantarum condenda, ex Floribus Balaustium, ex fructu Granatum, ex cortice fructûs Malacorium? Sit mens Sana in Sano corpore.

Erronea Nom. Specif. pronunciamus esse omnia qux vires, aut usum in se comprehendunt, sive ea $a b$ officinis, sive a salubritate, sive a Diæta, sive $a b$ œconomicis, s. ab Historicis notam acceperunt. 
a Pharmacopolio.

a Viribus.

Agrimonia officinarum C. B. Menyanthes antiscorbutica.

Calamintha officinarum Germa-Rhamnus catharticus.

nire C. B.

Solanum somniferum.

Martagon Alchimistarum Lob. Solanum furiosum.

Hieracium usuale $\mathrm{Rd}$.

$$
\text { a Vitâ. }
$$

Solanum Lethale.

Aconitum Salutıferum. ab Oeconomicis

Genista scoparia.

Rubia tinctoria.

Dipsacus fullonum.

Ricinoides, ex qua paratur Tournesol Gallorum T.

Pisum cortice eduli.

Pisa, quæsimul cum folliculis comeduntur Volk. ab Historiâ.

Punica, quæ Malum Granatum fert.

Canellifera arbor, cortice $i-$ gnobiliore, cujus folium Malabatbrum officinarum.

270. Sexus nullibi species diversas constituit unquam.

Intelligitur heic Sexus Dioicus maris, et feminæ, non verò sexus Monoicus, Hermaphroditus \&c. ex. gr. in Rumice, Species Acetose mares, et feminæ, s. Dioicæ sunt; Beta spinosa Androgyna, seu Monoica; Lapatha Hermaphroditæ, seu Monoclinæ; Acetosa Alpina digyna. Has notas inservire pro differentiâ specificâ nullus negavit.

Auctores plurimi J. B. Raj. Tournef. constituerunt ex sexu maris, et feminæ diversas species, quas, uti solo sexu differentes, negamus esse specie distinguendas.

e. gr. Urtica Mas, et Femina.
Cannabis Mas, et Femina.
Humulus Mas, et Femina.

Erronee ob sexum distinctum veteres distinxerunt species, ubi sexus diversus non fuit, sed diversissima planta, ex. gr.

Mas, et Femina:

$\begin{array}{lll}\text { Anagallis } & \text { Cistus } & \text { Orchis } \\ \text { Aristolocbia } & \text { Cornus } & \text { Pronia } \\ \text { Abrotanum } & \text { Crista galli } & \text { Pulegium } \\ \text { Abies } & \text { Ferula } & \text { Quercus } \\ \text { Amarantus } & \text { Filix } & \text { Symphytum } \\ \text { Balsamina } & \text { Mandragora } & \text { Tilia } \\ \text { Caltha } & \text { Nicotiana } & \text { Veronica. }\end{array}$


27. Monstrosi Flores ( $15^{\circ}$ ), et Plantæ a Naturalibus originem omnes trahunt.

Flores Multiplicatos, Plenos, et Proliferos diximus $\S$. I 19 . I 20. I 2 I. I 22.essemonstrosos $\$$. I so.et or tos a simplicibus.

Distinguimus Creatoris artificio distinctas species a Naturæ ludentis varietatibus.

Fiunt hæc monstra frequentissimè a culturæ mangonio, et nimio alimento.

Monstra animalium nullus pro distinctis speciebus unquam sumsit, ergo nec plantæe sumantur.

Demantur e Botanicâ Flores majores, multiplicati, pleni, proliferi, et exulabit numerosa Grex, quæ Botanicem diu oneravit.

272. Pubescentia (1 36 : vili.) ludicra est differentia, cùm culturâ sæpius deponatur.

Spinas, et Hirsutiem hic sub pubescentiâ intelligimus, quar plantæ sæpius exuunt a loco, vel culturà.

Cultura facit, ut miro modo mansuescant ferocissima animalia, quod idem et in plantis evenire quotidie observamus.

Urus ferox fit Taurus mitis.

Canis sylvestris ferox fit Canis mansuetus.

Spinose arbores culturâ sæpius deponunt Spinas in Hortis; et ex acerbo fructu mitem porrigunt. e. gr. Pyrus, Citrus, Limon, Aurantium, Mespilus, Oxyacantba, Grossularia, $\mathrm{Cy}_{-}$ nara.

Cichorium sylvestre est foliis sinuatis dentatis asperis, sapore amaro ingrato.

sativum est foliis magis integris, lævigatis, sapore grato.

HiRsuties loco, et ætate facillimè deponitur.

Fagus primò e semine, vel gemmis enata, hirsutissima:

mox glabra evadit.

Heliocarpus tenella foliis tomentosis est, adultaverò glabris. Triumfetta pusilla tomentosa est, adulta hispida. Asperula odorata in sylvis villosa, in apricis scabra fit. Persicaria amphibia in aquis glaberrima, in siccis scabra. Thymus serpillum in campis glabra, in maritimis arenosis hirsuta.

Scabiosa succisa in apricis glabra, in sylvis subhirsuta. Plantago Coronopus in humido foliis glabris integris, in sicco hirsutis dentatis. 
Lilium Martagon in sylvis hirsutum, in hortis glaberrimum.

Alchemilla palmata in apricis exsuccis glabra flavescens, in spongiosis umbrosis viridis hirsuta.

Clima mitius facit plantas mitiores, uti durius easdem duriores reddit, et sxpe quasi pelle induit. Ad hirsutiem itaque, et spinas non nisi summâ necessitate cogente confugiendum est:

Pentaphylloides palustre rubrum, crassis, et villosis foliis, suecicum, et hibernicum. Pluk. Bugula non crenata tomenzosa suecica Pluk.

273. Duratio sæpe magis ad locum, quàm ad plantam pertinet; in differentiâ itaque eam adhibere non arridet.

Calidæ Regiones, quæ æstate perenni gaudent, plantas alunt toto anno vix perituras; hinc plurimæ in illis regio= nibus plantæ perennes, et arborescentes evadunt, quæ apud nos annuxe sunt, uti Tropreolum, Beta, Majorana, Malva arborea \&c.

Frigidx regiones e perennibus plantis annuas faciunt, uti Mirabilem, Ricinum \&c,

A Duratione itaque, nisi manifestissima sit, nulla differentia petenda.

274. Multitudo Herbæ sæpe loco mutatur.

Caulis repens radiculas exserendo plerumque sese immensè multiplicat.

Multiplicantur plantæ vel in solo, vel in radice, vel in caule, vel in foliis, vel in fructificatione.

Frequens, et Vulgaris dicitur planta, quæ in solo apto sponte, et copiosè provenit.

Cespitosa planta fit, cùm multi caules ex eadem radice prodeunt; hoc minùs constans, cùm cxspitosa ceteroquin planta in solo macro unicum caulem difícilè progerminare valet; e contra caulis versùs radicem truncatus, in plantâ, quæ unicum assolet producere, tum plurimos promit instar hydræ.

F asciut a dici solet planta, cùm plures caules connascuntur, ut unus ex plurimis instar fasciæ evadat, et compressus. Fit idem arte, si plures caules enascentes cogantur penetrare 
coarctatum spatium, et parturiri tanquam ex angusto utero; sic sæpe in Ranunculo, Betâ, Asparago, Hesperile, Pinu, Celosiâ, Tragopogone, Scorzonerâ, Coiulâ fatidâ. Beta lato caule C. B. Amarantus cristatus.

Plicata dicitur Planta, cùm arbor, vel ramus excrescit minimis intertextis ramulis, tanquam Plica polonica ex pilis, ceu instar nidi Picæ, quod vulgus a malo genio ortum arbitratur; frequens a pud nos in Betulâ præsertim Norlandia, in Carpino Scaniz, nec infrequens in Pinu. Crispa 83. 63: folia fiunt, cum foliorum peripheria augetur, ut circumcirca fluctuet quasi undatus limbus.

Bullata folia fiunt ex rugosis, cùm discus (non a nobitus). multiplicetur, ut inter rugas adscendat substantia instar conorum, subtus concavorum: uti plurimæ Salvire, Ocymum.

Flores muliplicatos, plenos, proliferos ex simplicibus oriri, ex antecedentibus (I I 9-I 22. I 50-27 I) patet. Varietates loco specierum sunt variæ plantæ, quæ numero auctæ sunt.

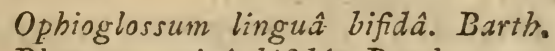
Plantago spicâ bifidâ. Barth.

275. Radix (8I) differentiam realem sæpe subministrat: ad eam tamen, nisi omnes alix interclusæ sint viæe, non confugiendum est.

Si alia supersit constans nota, ad radicem non confugiendum; non enim licet facilè in hortis plantas eradicare; in Herbariis non facile transfertur tadix ; in vivis plantis oculis nostris se subducit etiam radix.

Quò faciliùs dignoscuntur plantæe, eò meliùs, sed necessitati nulla lex posita est.

Scille difficillimè ab herbâ distinguuntur, a Bulbo tunicato; aut solido, aut squamoso facillimè.

Orcbidestutonan distinguuntur, nisi radices, furose, subrotunde, aut testiculate in auxilium vocantur. Fumarice bulbosæ radice c.zvâ majores, et minores.

radice non cavâ majores, et minores sunt tantùm varietates, ut monstrat habitus totius plantæ; huic etiam calyx vix oculis manifestus, et bracteæ a reliquis diversæ. 
276. 'TrUNCr (82) notæ differentias sæpe optimas eduat.

Caulis in multis plantis ita essentiales præbet differentias, ut eo demto nulla certitudo speciei.

Ciulis angulatus plures species distinguit, vix aliter distinguendas.

Hypericum Androscemum Fl. suec. 626. caule tereti. Hypericum perforatum Fl. suec. 625. caule ancipiti. Hypericum Ascyron Fl.suec.624.caule quadrangulari Convallaria Polygonatum vulgare Fl. suec. 247. a caule ancipiti.

Convallaria Polygonatum maximum Fl. suec. 247.6 caule tereti est.

hinc posterior Convallaria foliis alternis, caule tereti, pedunculis multifloris; prior Convallaria foliis alternis, caule ancipiti, pedunculis unifloris dicenda est. Hedysarum Fl. Zeyl. 286. caule triquetro notabilis. Lupini species vix nisi per caulem, aliis simplicem, aliis compositum, distinguerentur.

Culmus quinquangularis, et sexangularis est optima differentia Eriocauli Fl. Zayl. 48. 49. 50.

Scapus triqueter distinguit Pyrolas F1. suec. 337. 332 . a reliquis.

Petioli alati, s. latere utrinque membranâ aucti, pulchrè distinguunt Aurantium a congeneribus, et Hedysarum F1. Zeyl. 286.

Pedunculi diphylli, s. foliolis duobus oppositis instructi, iisque capitulo subjectis, eleganter Gomphrenam Fl. Zeyl. 1 Is. determinant.

277. Folra $\left(8_{3}\right)$ elegantissimas, naturalissimas differentias exhibent.

Natura in nulla parte magis fuit polymorpha, quàm in foliis, quorum itaque species numerosissimæe studiosè a Tyronibus addiscendæ.

Commendant se Folia pro differentiâ, quòd speciosissima sint, quòd species facie diversissimas efriciant, quòd facillimè differentias subministrant; hinc a foliis plurimas meas differentias in nominibus specificis mutuatus sum, uti patet conferenti Hortum Cliffortianum, Upsaliensem, 
Floram Suecicam, Lapponicam, Zeylanicam : his adde Gronovii Virginicam, Koyeni Leydensem, Guettardi Stampensem, Dalibardi Parisinam.

Characteres foliorum dedimus in aniecedentibus $\$ .83$. Rariora plurima occurrunt folia, quæ specialia sunt, adeoque nec sub generali notione veniunt.

Cucullatum folium, cujus latera basim versùs arctè conni-

vent, supernè explicata. Geranium afric.

Glandulosum, quod glandulas insidentes gerit :

in serraturis: Salix, Persica.

in dorso: Urena.

Acerosa folia formam aciculæe gerunt, subulata, et plerumque basi articulatione ramo inserta, uti Coniferce. Radicata folia, quæ Radiculas demittunt e substantiâ ipsius Folii.

Coadunata, quæ invicem annectuntur, ut basi concrescant.

Decussata ex Oppositis ( 83 : I I 2.) profluunt, ut si planta verticaliter inspiciatur, folia quadrifariam vergunt. Synonyma foliorum varia supersedemus.

Incisa, s. Dissecta, vide Laciniata p. 43. n. 28 .

Umbilicata quibusdam, vide peltata p. 48. n. I 19.

Cuspidatu, vide Acuminata p. 44 . n. $3^{8}$, sed acumine rigidiuscula.

Aspera, vide Scabra p. 45 . n. 55.

Strigosa, vide Hispida p. 45. n. 54.

Pulescentia, vide Pilosa p. 45. n. 53. sed parùm pilosa. Incuna, vide Tomentosa p. 45. n. 51. Folia, qux colorem glaucum habent, et fere argenteum, quod ex superficie singulari oritur.

Arrecta, vide Erecta p. 49. n. I 3 I.

Stricta, vide Recta, sed auget significationem, scilicet rectissima.

Assurgentiu, arcuatim erecta, s. primùm declinata, versùs apicem demum erecta.

Declinata, deorsum inflexa instar carinæ Naviculæ. Intelliguntur varia folia per se ex foliorum datis characteribus.

Bina prout Terna, Quaterna p. 48. n. I I I.

Enervia opposita Nervosis p. 45.11 .67$. Avenia opposita $V$ enosis p. 45. n. 66. 
Obversa, s. Verticalia, quorum basis angustior, ita ut basis concipiatur ubi nunc apex : e. gr.

Obversé-ovata, s. Obversé-cordata.

Verticaliter ovata, s. Verticaliter cordata.

Latina, quæ per se ex propriâ, et usitatissimâ lingua intelliguntur.

Trapeziformia, Rbomboidea, ex Mathematicis. Perennia, Biennia, Annua, a duratione in plantâ.

278. Fulcra $\left(8_{4}\right)$, et Hybernacula (85) communiter optimas differentias relinquunt.

- Hisce notis destitutus Botanicus, vix, ac ne vix quidem species facilè, et tutò determinabit, quod plurimis exemplis patet.

Aculei in Rubo. Spine in Pruno maximè notabiles sunt. Bractere in Fumariâ, Hedysaro indico, Dracocephalo.

Cóma constat bracteis, qua caulem terminant magnitudine insignes, ut in Coronâ Imperiali, Lavandulâ, Salviá.

Glandule in Pado, Urenâ, Mimosâ, Cassiâ, essentiales notas dant.

$\therefore$ Serrature glandulose ad basim foliorum in Heliocarpo, Salice, Amyglalo.

Dorsum foliorum glandulosım in Pado, Urenâ, Passiflorâ. Aculei glandulosi apice secernentes liquorem in Baubiniâ aculeatâ.

Glandularum cognitione destitutus nunquam certò, et tutò species distinguit in plurimis Generibus, præcipuè in Cassiâ, Mimosâ, aliisque.

Amygdalus a Persicâ solis glandulis serraturarum distinguitur.

Urence species nullus determinavit, antequam foliorum glandulas examinavit.

Convolvuli calyce tuberculato species in plures dirimeren- tur ob foliorum diversam figuram, nisi Glandulæ conjungerent.

Monardx corollâ glandulis adspersâ, evidentissimè distincta evadit a congeneribus.

StIPUl magni faciendre sunt in vastissimis Generibus, ubi dubium de Specie exsistat. 
Melianthus alia stipulis solitariis, alia varò geminis distinguitur.

Cassia stipulis reniformibus barbatis, Fl. Zeyl. I 5 I. distinctissina evadit a congeneribus omnibus.

Genme in eodem Genere sæpe diversissimæ, uti constat ex Genere Rhamni, ubi C'ervispina, Alaternus, Paliurus, Frangula, gemmis diversæ sunt.

Sulicum familia amplissima, et intricatissima, ex Gemma-

rum structurâ, et Foliatione facillimè, et certissiriè distinguitur in species corisiantisimas.

Buler distinguunt optimè, et ferè unicè Scilla Genus.

In axillis Foliorum residentes Bulbi Dentariam, Lilium, Ornitbogalum, Saxifragam, et Bistortam, singulari notâ determinant.

279. InFLoriscentia ( $163: \mathrm{XI}$.) maximè realis est differentia.

Inforescentia est modus, quo Pedunculus fructificationem promit (p. I I 2.), vel quoad structuram, vel locum; hoe alii Situm Fructificationis dixere, et ex eo hariolantes, nova Genera formarunt.

Pulcherrima inter omnes differentias mihi semper visa est Inforescentia, in plurimis generibus.

Spireer aliæe iloribus duplicato-racemosis, alize fl.corymbosis, aliæ floribus umbellatis, ut demtà hac notâ nulla certitudo speciei.

Pedunculus, qui flores promit, eosdem variè porrigit. Flaccidus est, cùm ita debilis, ut a proprio floris pondere dependeat.

Cernuus, cùnn apice incurvatur, ut flos versùs latusalterum, vel terram nute; , nec poterit erectus attolli ob curvaturam strictam pedunculi : uti in Carpesio, Bidente radiatâ, Carduo nutante, Scabiosâ alpinâ, He- liantino annuo, Cnico sibirico.

Fastigiati fiunt flores, cum petioli ita attollunt fructificitiones iñ fasciculum, ut supernè æquales altitudine evadant, ac si horizontaliter detonsi essent: exemplo Dianthi, Silenes.

Patulus petiolus ramulos undique dispergit, ut remotos ferat flores, oppositus coarctato. 
Congloinerait fiores fiunt, cum petiolus ranosus flores ferat absque ordine arctè congestos, et compactos, adeoque oppositi Panicule diffusre.

Articulatus, qui articulo unico instruitur: Oxális, Sida, Hibiscus.

Bini Pedunculi simul exeunt in Caprariâ, et Oldenlandia zeylanicâ.

Tcrni pedunculi ex eadem axillâ in Impatiente zeyl.

Flexuosi, s. Undulati Pedunculi in Airâ Fl. Suec. 64. et Poâ zeyl. 46.

Restantes in plantâ pedunculi post casum fructificationis: in $\mathcal{J}$ amboliferâ, Ocbnâ, fusticiâ.

Incrassati versùs florem pedunculi in Cotulâ, Tragopogone, et plerumque in floribus cernuis.

280. Fructificationis partes sæpius constantissimas differentias subministrant.

Olim aliter sentii, nec fructificationem, nisi præclusis aliis viis, adii, ob rationes, quòd brevis ævi esset flos, et quòd partes ejus sæpius minutissimæ.

Sunt in fructificatione plures partes, quàm in totâ reliquâ plantâ; adeoque plures inde notæ hauriuntur.

Minimis partibus, per totum naturæ campum, certitudo omnis innititur, quas qui fugit, pariter naturam fugit. Distinguendæ sunt fructificationis notæ in Essentiales, Naturales, er Specificas.

Tyro primùm dissecans flores, observat speciales notas, format nova, et falsa genera; credit se primùm flores vidisse, sed maturus Botanicus factus, se sæpius elusum sentiet.

Gentiance demto flore nequeunt distingui, uti constat observatis $\mathrm{Cl}$. Halleri, at corollæ in aliis Campaniformes, Rotate, Infundibuliformes; Quinquefida, Quadrifidr, Octofida facillimè distinguunt.

Hypericum floribus Trigynis a Pentagynis probè distinguendum.

Gerania africana dicta corollâ irregulari, staminibus conne$x$ is: debent a congeneribus europæis separari.

Lichenibus Tuberculum est fructificatio constans punctis scabris ex pulvere quasi congestis.

Scutellum est fructificatio orbiculata concava : margine undique elevato. 
Pelta est fructificatio plana, margini folii plerumque adglutinata.

Muscis Capitulum est Anthera.

Fungis Pileus est orbiculus horizontaliter expansus, qui subtus fructificationes gerit.

Graminibus Spicula est partialis spica, quam Locustam dixere alii.

Tortilis est arista cum geniculo contorto in medio notetur. e. gr. Avena.

Articulus est culmi pars Geniculis duobus interjecta. Compositus Radiatus flos constat disco, et radio.

Radius ex corollulis irregularibus in ambitu.

Discus ex corollulis minoribus plerumque regularibus constat.

Decompositus flos, s. Compositus ex compositis, continet intra calycem communem minores calyces communes multis floribus : Spherantbus.

Corolla AEqualis, ubi partes corollæ figurâ, magnitudine, et proportione aquales sunt.

Inæqualis, ubi partes non magnitudine, sed proportione respondent, ut regularis flos evadat: Butomus.

Regularis est æqualis figurâ, magnitudine, et proportione partium.

Irregularis, quæ limbi partibus, figurâ, magnitudine, aut proportione diversa est.

Rictus est hiatus inter duo labia.

Faux est hiatus tubi corollæ.

Palatum est gibbositas prominens in fauce corollx.

Calcar est nectarium ex corollâ. pone in conum extensa.

Urceolata, quxe urceoli, s. pelvis instar inflata, et undique gibba est.

Cyathiformis, cùm ex cylindro supernè parùm dilatala est.

Connivens, cujus limbi lobi apicibus convergunt.

Lacera, cujus limbus tenuissimè dissectus est..

Anthera versatilis, et incumbens, quæ latere affigitur.

erecta, quæ basi affigitur.

Pericarpium Inflatum, cùm instar Vesica cavum sit, nec repletur seminibus: Fumaria cirrhosa.

Prismaticum, cùm lineare polyedrum sit, lateribus phanis. 
Turlinalum, cùm fructus basi angustatur: Pyrus.

Contortum, cùm spiraliter contorquetur: Ulmaria, Helicteres, Thalictrum.

Acinaciforme, cùm fructus instar cultelli compressus, altero angulo longitudinali obtuso, altero acuto est. Mesembryanthemum, Dill.

Nidulantibus Seminibus T. cùm semina in Pericarpio baccato per pulpam dispersa sunt.

Echinatum, cùm aculeis, s. spinis undique adspersum, uti Echinus animal.

Torosum, cùm torulis, s. prominentiis hinc inde gibbum evadit; Lycopersicum T. Pbytolacca.

28r. Notæ genericæ (192), in differentiâ usurpatæ, absurdæ sunt.

Notas genericas Characteris Naturalis hic intelligimus, quæ species nunquam distinguunt; hæ enim in genere consentientes sunt per omnes species; nec dissentiunt, quæ consentiunt.

- Erroneas itaque esse omnes differentias statuimus, qux notas specificas desumunt a notis characteris naturalis.

Polygala siliculosa tetrapetala bicapsularis. Moris.

A ponogeton siaminibus singularibus. $\mathrm{P}$.

Guajacum Aceris, s. Burse pastoris fructu. Br.

282. Differentia omnis e Numero, Figurâ, Proportione, et Situ variarum plantarum partium (80-86) necessariò desumatur.

Notæ fallaces, et constantes unde promanant, diximus in

_. precedentibis.

Fallaces notas statuimus plurimas; uti
Color \$. 266 .
Tempus $\$ .265$.
Varians \$. 259.

Odor $\$ .267$.

Locus \$. 264. Accidentale $\$ .258$.

Sapor $\$ .268$. Multitudo \$.274. Auctoritas $\$ .263$.

Usus $\$ .269$. IIIagnitudo \$. 250 . Insufficientia $\$ .257$.

Sexus $\$ .270$.

Pubescentia $\$ .272$. Monstrositas $\$ .271$. Collatio $\$ .261 .263$.

Fid̆as notas desumendaś tantùm esse a partibus plantæ, uti a

Radice \$.275. Foliis \$.277. Inflorescentiâ \$. 279. Trunco\$. 276. Fulcris \$.27.8. Fructificatione $\$ .280$. 
Modi, quibus differentia desumitur, sunt quatuor:

Numerus, Figura, Situs, Proportio; adeoque iidem, qui in genere $\oint$. I67.

Hi ubique constantes, in Plantâ, in Herbario, in Icone.

283. Ne varietas ( 158 ) loco speciei ( I 57) sumatur, ubique cavendum est.

Hoc opus, hic labor est ; adecque summâ industriâ inquirendum.

Errores gignunt, ut sæpius cæcutiamus, causæ imprimis sequentes:

1. Natura polymorpha, in suis operationibus nunquam cessans.

2. Regionum, et Climatum diversa, et singularis natura.

3. Loca natalia remotissima.

4. Brevitas vite humanæ, quæ perit præcoci fato. Certitudinem detegunt in speciebus a varietatibus distinguendis:

Cultura in diversissimo, et vario solo.

Partium plantæ omnium examen attentissimum.

Fructificationis examen, quoad omnes partes etiam minutissimas.

Congsnerum specierum inspectio.

Nature leges constantes, nunquam saltus facientes. Varietatum modi remoti.

Positio speciei sub proximo, et diverso genere.

284. Nomen genericum singulis Speciebus applicari debet.

-r Speciebus ad Genera redactis, adsumant Species nomen generis, ut ex nomine constet de plantæ nominatæ genere.

Nomen Genericum valet in Republicâ Botanicâ,uti nummus. Rajus, et Morisonus sæpius reduxere Species ad Genera, sed nomina Generis non assumsere.

$$
\text { Stсенаs. Rij. hist. } 280 .
$$

I. Stcechas citrina germanica, latiore folio.

2. Chrysocome athiopica, plantaginis folio.

3. Helichrysum abrotani femine foliis.

4. Elichrysum creticum.

5. Stachadi citrina alteri inodore Lobelii affinis.

6. Gnaphalium montanum album. 
Sic, qui citatum Raji nomen audit, de genere nullam ideam habet, visi viso libro.

285. Nomen specificum semper genericum sequi oportet.

Cùm, ignoto genere, nulla certitudo; necesse est, ut Generis nomen incipiat ideam, si distinguat distinguenda. Loberivs maximè, et sæpius hic peccavit:

Minus Heliotropium repens. Lob.

Mattbio!i secundum Limonium. Lob. Aqustica Plantago foliis Beta. Lob.

286. Nomen specificum sine generico est quasi campana sine pistillo.

Differentia est tantùm distinctio generis, adeoque sine genere nulla differentia concipi potest.

Nomina sunt ex arte confecta, ut scientificè determisent plantas.

Diffe:entiæe sine nomine specifico sunt animalcula capite mutilata.

- myagro affinis berba, capsulis subrotundis J. B.

- linarive aliquatenus similis birsuta non laciniata C. B.

- linarie aliquatenus similis, folio bellidis J. B.

- periclymeno accedens planta monantbos Mor.

- indigena alpium Sabaudie Bocc.

287. Nomen specificum ipsi nomini generico adglutinatum non erit.

Fuere Botanici, qui genera per caudas distinguere tentarunt, inflectendo nomen in fine, sed peperit confusionem summam.

Distinctum genus; Distincta Differentia. Gentianeila pro Gentianà parvâ. Acetosella - Acetosá parvâ.

288. Nomen specificum genuinum est vel Synopticum, vel Essentiale.

Nomina specifica citò, tutò, et jucundè distinguant species. Selectus fiat ex omnibus differentiis possibilibus speciei, unde desumendæ præstantissimæ, ut liceat tandem tutò nosse speciem. 
Modus Nominum specificorum est vel Synopticus, vel Essentialis, vel ex utrisque mixtus.

289. Nomen specificum Synopticum (288) plantis congeneribus ( 159 ) notas semidichotomas imponit.

Ubi notæ essentiales speciei investigari nequeunt, necesse est, ut per Synopsim fiat differentia, adeoque Synopsis est succedanea differentiæe essentialis.

In vastissimis generibus sepius ad synopsim adsumendam coacti sumus.

Salix foliis serratis glabris osatis acutis subsessilibus.Rcy. Synopticé.

Salix fosculis pentandris. Flor. lapp. Essentizle. Salix foliis subintegerrimis lanceolato-'inaaritus longissimis acutis: subtus sericeis, ramis virgatis. Fl. suec. Syroptice.

Salix foliis linearibus revolutis. Essenizale.

290. Nomen specificum Essentiale (288) notam differentiæ singularem, suæve speciei tantummodp propriam, exhibet.

Nomen specificum essentiale absolvitur unico, alterore vocabulo, s. unicâ ideâ.

Stabilitis Generibus, et Speciebus essentiali differentiả determinatis, summum apicem in re Herbaria tetigimus. Si Botanici eò tandem perveniant, ut omnes species no mine Essentiali determinare queant, non datur ulira.

Præstantia nominis commendat se brevitate, facilitate, certitudine.

Valent nomina essentialia absque citatione, quod alia nomina nunquam.

Synopsis, detecto nomine essentiali, non admittenda est in differentiâ specificâ; allaborandum itaque, ut aর̉ metam tandem perveniamus propositam.

Eriophorum spicis pendulis Flor. lapp. 22.

Plantago scapo unifioro Fl. lapp. 64.

Alchemilla foliis simplicibus F1. lapp. 66.

Alchemilla foliis digitatis Fl. lapp. 6-.

Menyanthes foliis ternatis Fl. lapp. 80.

Convallaria scapo nudo Fl. lapp. I I 2.

Convallaria foliis verticillatis Fl. lapp. II 4 .

$\mathrm{P}_{2}$ 
Pyrola scapo unifioro Flor. lapp. I67. suec. 33.4. Betula foliis orbiculatis crenatis F1. lapp. 342. suec. 777 .

29r. Nomen specificum qù̀ brevius, eò etiam melius, si modò tale (257).

Pulchritudo artis brevitatem exposcit, nam quò simplicius, eò etiam et melius, et stultum est facere per plura, quod fieri potest per pauciora; Natura etiam ipsa compะndiosissima est in omni suâ actione.

Numerus vocabulorum, quæ in differentiâ adhibentur, nunquam ultra duodecim vocabula admittat; quemadmodum Nomina Generica duodecim ad summum literis constabunt $\$$. 249 . it a et differentia I 2 vocabulis, ut limites tandern stabiliantur.

Duodecim vocabula suficicere pro differentiâ specificâ ex sequenti calculo elucescit. Sit genus centum specierum, quem numerum adtigisse nullum adhuc cognitum genus novimus; distinguantur hx species synopsi: a. 50.b. 2 5.c. I 3.d. 7. e. 3. f. 2. g. I. adeoque sex substantiva cum totidem adjectivis ad summum necessaria essent; at verò unum idemqque substantivum sxpe admittit plura adjectiva; adeoque duodecim vocabula vix unquam necessaria erunt pro differentiâ generis centum specierum.

Horrenda itaque sunt nomina specifica veterum sesquipedalia, quie descriptiones loco differentiarum sistunt.

Cenchramidea arbor saxis alnascens, obrotundo pingui folio, fructu pomiformi in plurimas capsulas grana ficulnea (stylo columnari hexagons preduro) adherentia continentes, diviso, Balsamum fundens. Plukn. alm. 92.

Gramen myloicopbo-on carolinianum, s. Gramen altissimum, $p a-$ niculâ maximâ speciosâ, e spicis majoribus compressiusculis utrinque pinnatis, blattam molindariam quodammodo referentibus, compositâ, foliis convolutis inucronatis pungentibus. Pluk. alm. 173.

Acacix quodammodo accedens, Myrobalano chebule Veslingii similis arbor americana spinosa, foliis ceratonice in pediculo geminatis, siliquẩ bivalvi compressâ corniculat â, seu cocblearum, vel arietinorum cornuum in modum incurvat $\hat{\text {, }}$ sive Unguis cati. Breyn. prodr. 2. p. 29. 


\section{DIFFER E N T IA.}

292. Nomen specificum nulla admittat vocabula, nisi quibus a congeneribus necessariò distinguitur.

Nullum vocabulum in differentiâ specificâ superfluum erit. Si eadem nota poterit paucioribus verbis exprimi, erit illa optima, quæ brevissima fuit.

Exulent Antonomasia, Tautologia, et flosculi oratorit. Betula nanx pumila Franken. Tautologia.

Lamium caule folioso Lind. Antonomasia.

293. Nomen specificum nullum speciei in suo genere solitariæ (203) imponi potest.

Sunt qui statuunt nomen specificum addi debere speciebus novi generis, licèt solitariis, ut inde formetur idea plantæ. Non negamus Habitum multum inservire ad formandas notiones de plantâ ; at verò in nomine specifico admodum erronea est, cùm contineat nihil aliud, nisi solam notam, qua distinguatur a congeneribus.

Ubi nulla differentia specifica ad plantam nominatam, ibi præsupponitur in genere non nisi unicam speciem esse detectam.

Erronea itaque sunt nomina specifica, quæ differentias imposuere plantis in suo genere solitariis pag. $136 . \mathrm{e} . \mathrm{gr}$. Morina orientalis, carline folio. Tournef. cor. 48 .

Dalechampia scandens, lupuli foliis, fructu bispido tricocco. Plum. Amer. 17.

Matthiola folio aspero subrotundo, fructu nigricante. Plum. Amer. 16.

Maranta arundinacea, cannacori folio. Plum. amer. I6. Valdia cardui folio, fructu subcreruleo. Plum. amer. I I.

294. Nomen specificum imponat, qui novam adinvenerit speciem, si modò necesse sit (293) tale.

Qui novam detegit speciem, addat et non modo ejusdem differentiam, sed et in congeneri vel congeneribus differentias augeat, ut distinguantur in posterum species sufficienti differentiâ.

Chavtonia Gron. virg. 25. primùm innotuit in Virginiâ; dein alia species Limnia dicta in Sibiriâ : adeoque dicatur Sibirica: Claytonia foliis ovatis, et Virginica Claytonia foliis linearibus. 
295. Nominis specifici vocabula non erunt composita, nominibus genericis similia, nec græca, sed tantùm latina; nam quò simpliciora, eò etiam melioran.

Character definit nomen Genericum, Differentia verò specifica se ipsam; adeoque prius esse poterit peregrinum, posterior autem per se evidentissima erit; ideo hrec purè latina, nec græca.

Erronex itaque sunt differentiæ specificæ omnes græcæ.. Lathyrus disioplatyphyllos. Lotus oligokeratos.

Myrrhis conejophyillon - - polykeratos.

Potamogeton lejopbyllon. Mimosa platykeratos.

_. - iteophyllon. _... - bracbyplatolobos.

- - malacopbyllon. Pisum leptolobon.

- - ulophyllon.

Pilosella monoclonos.

Lotus tetragonolobus.

- - polyclonos. Trifolium katoblebs.

Ficus aizoides.

Asclepias aizoides.

Hieracium piloselloides.

Oreoselinum anisoides.

Belleval, Prof. Monsp. circa finem seculi XVI. rarissimas icones, qux non prodiere, incidi curavit ; his mecum communicatis a Cl. Sauvagesio, intellexi Auctori in animum fuisse omnes differentias græcâ linguâ com$\therefore$ posito vocabulo exprimere; e. gr.

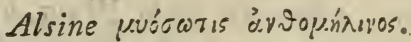

Androsaces igexatígraurov.

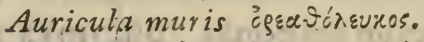

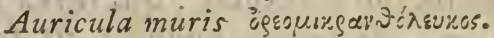

Betonica ¿รrop?

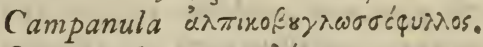

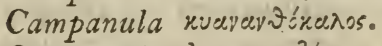

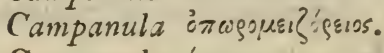

Campanula ó

Carduus àvóxay-sos.' I I a

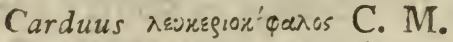

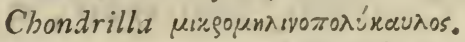

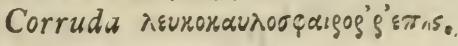




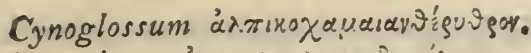

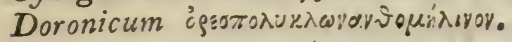

Gentianella éagaraudóseıs.

Glycyrrbiza paugop’i?

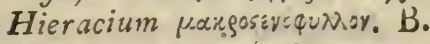

Hieracium $\pi \lambda \alpha \tau$ idil ov.

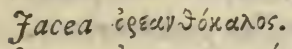

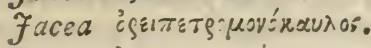

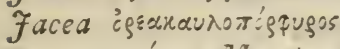

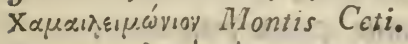

Nardus ęsitidóresiros.

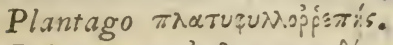

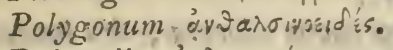

Pulsatilla civinutopuirivos.

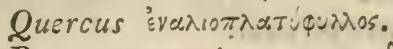

Racemus marinus oq̣ugoøı́丶万.

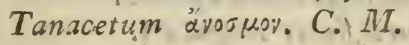

Thlaspi ipeoxaunopuniosecins Horti dei.

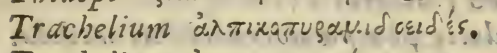

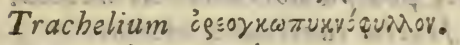

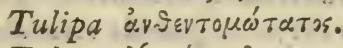

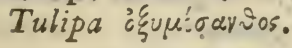

296. Nomen specificum non erit Tropis Rhetoricis figuratum, multò minùs erroneum; sed fideliter, quæ natura dictitat, exponat.

Synerdoche totius pro parte frequentissima est in Botanicis, - ubi de toto prædicatur, quod valet de parte tantumm;

quod nunquam admittendum esse censemus:

Synecdoche numeri singularis pro plurali tritissima, et xquè

erronea est.

Metaphora semper obscura est, hinc præstat nuda simplicitas.

Ironia cùm mendacii species sit, hinc excludenda erit.

Synecdoche totius pro parte.

Salicaria purpurea pro corollis purpureis.

Quinquefolium folio argenteo pro subtus albo.

Molucca spinosa pro calycibus spinosis.

Pimpinella umbellâ candidâ pro corollis candidis. 
Synecdoche numeri singularis pro plurali.

Lupinus flore luteo pro floribus luteis.

Ranunculus folio rotundo, et capillaceo pro foliis rotundis, et capillaceis:

- Metaphora.

Limon incomparabilis pro Mlaximus.

Caryophyllus superbus profloribus pulcherrimis.

Majorana gentilis pro odoratissima.

Tragon improbus pro aculeatus.

Ranunculus sceleratissimius provesicatorius.

Urtica fatua pro inermis.

Cannabis erratica pro mas.

Hesperis mslancholica pro noctu odorata, diu inodora.

Urtica mortua pro inermis.

Bulbonac. rad. redivivâ properenni.

Meum adulterinum pro non genuina species.

Orchis abortiva pro figurâ froris singulari.

Pinus incubacea pro fusciata.

Fucus hemorrhoidalis pro hemorrboidibus medela.

Pepo strumosus pro fructu tuberculis obsito.

Caryophyllus barbatus procalycis squamis setaceis.

Mentha cataria pro odore catis grato.

Gramen leporinum pro tremens, uti Lepus.

2i Laotuca agnina pro ovibus grat. species.

A parine semine saccharato pro fructu verrucoso.

- Arbor finium regundorum pro tinctura ad mappas inde colore distinguendas.

\section{Ironia.}

Lysinachia bifoliafi. globoso pro foliis oppositis, racemis ovatis.

Oputhogalum flore minore innato pro filamentis planis.

Narciśsis calyee tuteo pro nectario luteo.

Dentaria baccifera pro bulbifera ex alis.

Dracunculus pistillo longissimo pro receptaculo longissimo.

Frágaria sterilis pro receptacúlo sicco.

Sabina sterilis pro mas.

297. Nomen specificum nec comparativum, nec superlativum sit.

Comparativa M.zjus, aut Minus, gradus Comparativus, et Superlativus non admittenda. sunt, cùm præsupponant cognitionem alterius plantæ: 
Alsine altissima est minor Betulâ nanâ.

Contra autem sentiendum, ubi superlativ us gradus adhibetur de plantie presentis parte, et indicat partem esse maximam inter omnes plantæ partes; tum enim egregia nota est:

Lobelia pedunculis brevissimis, tubo corolle longissimo. Roy.

Erronea sunt nomina specifica omnia, quæ comparationem habent extra plantam.

Equisetum levius Raj.

Pilosella major, miniss birsuta C. B.

Pilosella minor, folio angustiore, minùs piloso I. B.

298. Nomen specificum terminis positivis, non verò negantibus, utatur.

Negativa nihil dicunt, vel dicunt quod non est, non verò quid est.

Quamdiu vocabula positiva adsunt, nunquam licet privativis uti, adeoque semper parata erunt vocabula, quæ ideas oppositas exprimunt.

Subrotundum, et oblongum.

Rotundatum - angulatum.

Simplex, et compositum.

Fissum - - - indivisum. Petiolatum - - - sessile.

Obiusum - - acutum.

Erecius - - - volubilis.

Pungens - - inerme.

Teres - - - - angulatus.

Serratum - - integerrimum. Aristatus - - - muticus.

Nervosuns - - enerve.

Tomentosum - glabrum.

Laxus (Haccidus',-strictus.

Tectum - - nulum. Remoti - - - conferti.

Venosum - - avenium. Divaricati - - coaritati.

Teres - - - angulosum. Caulesiens - - acaulis.

Tubulosum - - farctum.

Herbaceus-lignosus, s. fruticosus

Fiat descriptio plantæ vastissima terminis negativis, nemo indesibi formet idean levissimam plantæ. vide D. Episcop. Browallii Examen epicriseos.

Erronea itaque sunt omnia nomina specifica, quæ admittunt vocabula, vel particulas negativas.

Lysimachia non papposa Mor. Seminibus nudis.

Hippuris non aspera I. B. glabra.

Bidens folio non dissecto $T$. integro.

Phalangium non ramosum Wehm. caule simplici.

Lychnis petalis non bifidis Mar. integris. 
299. Similitudo omnis, in nomine specifico usurpata, dextrâ manu notior erit, licèt et hæc minùs placet.

Similitudo unico verbo exhaurit id, quod alioquin integrâ oratione demonstraretur; sed omnis similitudo claudicat, ideoque etiam in artis dedecus assumitur omnis similitudo obscura, et non unicuique manifestissima.

Assumenda itaque nulla alia similitudo, quàm a partibus externis corporis humani, uti Auris, Digitus, Umbilicus, Oculus, Scrotum, Penis, Vulva, Mamma ; non vero $a b$ internis corporis partibus, quæe anatomicis solùm notissimæ.

Similitudo valet sine definitione, quod nullus alius artis terminus.

Obscuras similitudines, et quidem plurimas, introduxere Botanici non omnibus notas, e. gr.

Agaricus tuba fillopiane instar $\mathrm{T}$.

Orchis antbropophora.

Orchis Simiam referens C. B.

Orchis Cercopithecum exprimens Col.

Orihis Muscam referens.

Mesembryanthem. rostrum Ardece referens D.

Mesembryanth. rictum caninum referens $\mathrm{D}$.

Mesembryanth. rictum felinum referens $\mathrm{D}$.

Lotus siliquis pedes corvinos referens C. B.

Fungus pilis Capreoli prorsus similibus Lœe.

Atriplex semine bucephali Col.

Arbor acul. ramis incurvis, scenam topiariam efformans. Plukn.

Fungus dedaleis sinubus $\mathrm{T}$.

Fungus auriscalpium referens.

Hemionitis foliu securis Romana figurâ. Pluk.

Medica caseiformis. Rudb.

300. Nomen Specificum nullum adjectivum sine opposito substantivo adhibeat.

Nullum adjectivum, adeoque nullum adtributum in nomine specifico, sine prægresso substantivo, cui respondeat; ubi autem nulla pars priùs nominata, ibi supponitur prædicari de totâ plantâ. 


\section{I F F E R E N T I A.}

Substantiva hic usitata semper erunt partes plantæ.

Erronea sunt omnia Nomina specifica, quæ admittunt adjectiva sine opposito substantivo.

Adjectiva sine substantivo vero.

Millefolium cornutuin C. B. foliis.

Nigella cornuta C. B. capsulâ.

Thlaspidiuns cornutum $T$. calyce.

Lysimachia corniculat a C. B. capsulâ.

Allium bicorne C. B. spathâ.

Viola tricolor C. B. corollà.

Myrtus cristata Wehl. foliis.

Amaranthus cristatus, spicâ.

Gramen cristatum. bracteis.

Solanum vesicarium. calyce.

Colutea vesicaria pericarpio.

Ranunculus vesicarius. vi.

Millefolium vesicatorium. radice instructâ vesiculis. Mesembryanth. vesicatorium. foliis adspersis.

Substantiva Erronea.

Sideritis utriculis spinosis Herm. pro verticillis. Adjectiva, et Substantiva genere conveniant.

Juniperus alpinus Clus. alpina.

Hippuris muscosus Mor. muscosa.

$30 \mathrm{r}$. Omne adjectivum (300) in nomine specifico sequi debet substantivum suum.

Quemadmodum in Charactere præcedat semper pars, seu priedicatum; ita et in differentiâ semper substantivum, cui adjectivum competit, ut fiat distinctissima idea, ne errore typographi, ob comma, vel punctum alienus sensus suboriatur.

Corona solis parvo flore, tuberosa radice $\mathrm{T}$.

Rectius: flore parvo, radice tuberosâ.

Sinapistrum ægyptium heptaphyllum; fore carneo, majus spinosum Herm.

REctius : ægypt. heptaphyll, majus spinosum, fore carneo.

Orchis æthiopica, maxima, maculata, aviculam niveam maculâ sanguineâ, in dorso, notatam repræesentans, galeâ cæruleâ, amplissimâ, pulvere argenteo, adspersâ in areâ, insigniter splendente. 


\section{6 \\ DIFFER EN TIA.}

Recrius : $x$ thiopica, maxima, maculata, repræsentans aviculam niveam: notatam in dorso maculâ sanguineâ, galeâ cæruleâ amplissimâ adspersâ pulvere argenteo.

302. Adjectiva ( 300$)$ in nomine specifico usurpata, e terminis artis selectis (80-86), si modo sufficientibus petenda sunt.

In Terminis artis si conveniant Botanici, et si constantes sint in scriptis, facillima evadet scientia,

Periphrasim nunquam admittat Botanicus, quamdiu Termini artis rite definiti occurrunt.

Synonyma Terminorum excludantur, et unicus constans, et selectus adhibeatur.

Lychnis viscosa
Lychnis glutinosa Rd. glutinosa.

Synonyma.

Caryophyllus supinus C.B.
Malva procumbens procumbens.

$\left.\begin{array}{l}\text { Ligustrum foliis pictis. Weh. } \\ \text { Laurocerasus foliis variegatis. Weh. }\end{array}\right\}$ variegatis.

Pilosella rep. hirsuta.

- - folio piloso

Quinquefolium pubescens. $S$

pilosa.

$\left.\begin{array}{l}\text { Hieracium radice succisâ } \mathrm{C} \text {. B. } \\ \text { Hieracium radice premmorsâ. Mor. }\end{array}\right\}$ præmorsâ.

Per Periphrasim.

Quinquefolium molli lamugine pubsscens. I. B. villosum, Conyza bumidis locis proveniens. I. B. palustris.

Muscus squamosus in aquis nascens. Moris. aquaticus.

303. Particulas, adjectiva, substantivaque, conjungentes, nomen specificum excludat.

Particulae ejusmodi sunt: Vel

Conjunctivæ: et, atque, simul.

Disjunctivæ: vel, sive, seu.

Casu ablativo, absque ullâ præpositione, notæ omnes in differentiâ proponantur.

Ubi autem duo diversa indicanda in eadem plantâ, utimur nota $v e$, vel que in fine sequentis vocabuli, ne augeat numerum vocabulorum: e. gr. 


\section{I F F.E R E N T I:A,}

Carduus foliis lanceolutis ciliatis integris hiciniatisquie. Hort. cliff. 392 .

Juncus foliis planis, spica sessili pedunculatisque:s Flor. suec. 288.

Exulant differentixe omnes, qux admittunt particulas dis-

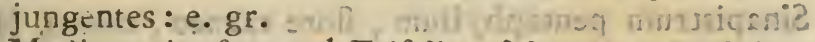

Medica sylv. frut. vel Trifolium falcatum, seu Medica siliquâ tortili. Moris.

Absinthium ponticum, seu romanum officinarum, seu Dioscoridis. Moris. C. B.

Aster montanus, sive Oculo Christi similis, si non idemí, sive Conyza 3. I. B.

304. Notæ distinctivæ partes plantarum (80), non verò adjectiva, in nomine specifico distinguant.

Particulæ distinguentes hujus loci sunt: Comma (, ), Comm.s cum puncto $(;)$, Colon $(:)$, et Punctum (.).

Differentia specifica per particulas rite interstinctas evidentissima evadit.

EGo utor commate ad distinguendum partes; Colon autem adhibeo, ubi partis subdivisio est; Puncto auten claudo differentiam. A.

Alir utuntur Commate cum puncto ad distinguendum partes; Commate verò distinguunt iidem omnia adjectiva. $\mathrm{B}$.

Antecessones Commate et partes, et adjectiva plerumque distinxerunt. C.

A. Bauhinia inermis, foliis cordatis semibifidis: laciniis acumina!o-ovatis erecto-dehiscentibus. H. C.

B. Bauhigia inermis ; foliis cordatis, semibifidis, laciniis acumiuato-ovaris, erecto-dehiscentibus. H. C.

C. Bauhinia inermis, foliis cordatis, semibifidis, laciniis acuminato-ovatis, erecto-dehiscentibus. H. C.

305. Parenthesis Nomen specificum nunquam admittat.

Parenthesis vel subintellecta, vel lineolis incarcerata, codem recidit.

Arguit utraque aut exceptionem, aut defectum ordinis, adeociue non admitterida est. 
Boerhaavius, et coætanei, ne innovarent nomina, antiquo nomini generico præposuere novi Generis nomenclaturam , cum interjectâ syllabâ qui, s. quee, s. quod: hoc autem ex datâ in præcedentibus theoriâ non assumimus.

a. Parenthesis subintellecta.

Sinapistrum pentaphyllum, flore carneo, minus. $\mathrm{H}$. b. Parenthesis adscripta.

Androsæmum maximum(quasifrutescens) bacciferum.Mor. Violæ affinis umbilicato (seu peltato) folio scandens. $\mathrm{Br}$. c. Qui, Qure, Quod.

Dens Leonis, qui Pilosella folio minùs villoso. $T$.

Doria, que Jacobæa orientalis limonii folio. T. cor. B. Titanokeratophyton, quod Lithophyton marinum albicans. Gesn. $B$. 


\section{V A R I E T A T E S.}

306. Nomini Generico (vir), et Specifico (vin.) etiam Varians, si quod $\left(15^{8}\right)$, addi potest.

Varietates sunt plantær ejusdem speciei, mutatæ a causâ quacunque occasionali.

Usus varietatum in Oeconomiâ, Culinâ, Medicinâ necessariam reddidit earum cognitionem in vitâ communi; ad Botanicos ceteroquin non spectant varietates, nisi quatenus Botanici curam gerant, ne Species multiplicentur, aut confundantur.

Evidentiores varietates, ob usum publicum, ad finem differentix inserat Botanicus, ubi necesse est.

30\%. Nomina Generica, Specifica, et Variantia literis diversæ magnitudinis scribenda sunt.

Nomen Genericum pingatur literis majoribus Romanis.

Specificum literis mediocribus, et vulgatissimis.

Varians autem literis minoribus currrentibus vulgò dictis.

Fiat hoc, ut Varietas distinctissima tradatur a Differentiâ. CONVALLARIA scapo nudo; corollâ plená. CONVALLARIA scapo nudo; corollâ rubrâ. SAXIFRAGA alpina ericoides; flore purpurascente. SAXIFRAGA alpina ericoides; fiore caruleo. PENTAPHYLLOIDES palustre rubrum; crassis, $\epsilon$ t villosis foliis,

308. Sexus ( r 49) Varietates naturales constituit; reliquæ omnes monstrosæ sunt.

Dioicæ plantæ constituunt unicum mcdum varietatum verè naturalem, in Mares, et Feminas distinctum; quas nosse, et differentiis addere, Botanicis perquam necessarium est.

$V$ eteres, ignari fundamenti foecundationis plantarum, Mares pro Feminis, et Feminas pro Maribus assumsere, quod sedulo vitandum: e. gr.

$\left.\begin{array}{l}\text { Mercurialis mas. T. } \\ \text { Cannabis mas. L. B. } \\ \text { Lupulus mas. T. }\end{array}\right\}$ sunt femina, 
309. Varietates monstrosas (303) constituunt Flores mutilati (1:9), multiplicati (r20), pleni (12 I), proliferi (222); Herbo luxuriantes, fasciatæ, plicatæ, mutilatæ: in numero, figurâ, proportione, et situ partium omnium, necnon sæpius Color, Odor, Sapor, et Tempus.

Modi primarii varietatum sunt sequentes:

Corollse Mutilatio $\$$. 119.18 4.

II Iultiplicatio \$. I 20. I 26. I 27. Implatio \$. I 2 I. I 25. Prolificatio \$. 123.124.

Caulis Luxuriatio Fasciculat. $\$ .274$. Plicata $\$ .274$. Foliorum Luxuriatio Crispa \$. 3 I I. Bullata \$. 311 .

Herbx Color \$. 266. Odor \$. 267. Sapor \$. 268. ITagnitudo \$. 260 . Tempus \$. 265.273.

Coloratio foliorum.

Buxus foliis per limbum aureis. $T$.

Aquifolium spinis, et limbis argenteis. $\mathrm{T}$.

Alaternus foliis ex luieo variegatis. H. R. P.

Salvia folits ex viridi, et huteo variegatis. H. R. P.

Aloë sobolifera foliis eleganter variegatis. Herm. prodr.

Caprifolium perfoliatum, foliis sinuosis, et variegatis. T.

- Urtiea urens minor folits ex viridi, et rubro éleganter variegatis. Rudb.

Gramen paniculatum aquaticum phalaridis semine, folio - variegato. T.

3ro. Varietates levissimas non curat Botanicus (7).

Anthophill, nimiâ industriâ, et assiduâ inspectione, in corollis florum ea miracula observarunt, qua inexercitatus oculus nequit perspicere; Objectum horum sunt flores pulcherrimi Tulipa, Hyacinthi, Pulsatille, Ranunculi, Dialm thi, Primule; Occultas varietates harum nominarunt vocabulis stuporem excitantibus; hi propriam scientiam florum colunt, adeptis solis manifestam; castra itaque eorum nullus sanus Botanicus intret.
Phecbus
Apolls
Astrae
Triumphus Flore.
Dredalus
Pompa Flore.
Cupido
Splendor Asic.
Corona Europe.
Gemma Hollandia. 
Aurora. Sponsa Amstelodami.

Gratiosa.

Pretiosa.

Alexander Magnus.

Carolus Duodecimus.

Julius Ceesar.

Imperator Augustus.

Tartar Cbam.

Hortulanorum vulgus Pomiferis, et Drupiferis nomina varietatum imposuere, nec delinita, nec definienda, et numero pæne infinita.

$\begin{array}{rc}\text { Poma Paradisiaca } & \text { Pyra Falerna } \\ \text { Prasomila } & \text { Favonia } \\ \text { Rubelliana } & \text { Boni Christiani } \\ \text { Barstorfiana } & \text { Crustanina } \\ \text { Appiana } & \text { Picena } \\ \text { Melimela } & \text { Libraria. }\end{array}$

Fungorus ordo in opprobrium artis etiamnum Chaos est, nescientibus Botanicis in his, quid Species, quid Varietas sit.

3I I. Foliorum 1uxuriatio in Oppositione, et Compositione facillimè accidit. Crispa, et ButraTA folia omnia monstrosa sunt.

Opposita folia $\$ .83$ : I I 2. sæpe evadunt Terna 83 : III, seu Quaterna 83 : I I I. et tum ex caule Quadrangulari fit caulis Polygonus:

Lysimachia lut. major, foliis termis. T.

Lysimachia lut. major, foliis quatermis. T.

Lysimachia lut. major, folias quinis. T.

Anagallis cærulea, foliis binis, ternisve ex adverso nascentibus. Raj.

Anagallis phonicea, foliis amplioribus, exi alverso g̨uaternis. T.

Salicaria trifolia, caule hexagono. T.

Digitata folia sæpe addunt unum, alterumve foliolum.

.Trifolium quadrifolium hortense album C. B.

CRISPis foliis plantæ sunt omnes monstrosæ varietates, uti Corollæx plenæ in floribus, adeoque plantæ his foliis instructie nullix naturales, sed omnes aliam pró matre agnoscunt.

Q Apium 
Apium, s. Petroselinum crispum. C. B. Heracleum : Sphondylium crispum. C. B. Rumex : Lapathum folio acuto crispo. C. B. Acetosa foliis crispis. C. B.

Reseda crispa gallica. Bocc.

Luteola lusitanica pumila crispa. $\mathrm{T}$.

Brassica laciniata rubra. J. B.

Nasturtium hortense crispum. C. B.

Malva crispa. J. B.

Lactuca crispa. C..B.

Cichorium crispum. T.

Lapsana folio amplissimo crispo. B.

Tanacetum foliis crispis. C. B.

Matricaria crispa.

Asplenium : Lingua cervina maxima, undulato folio. H. R. P.

Leonurus : Cardiaca crispa. Raj.

Mentha crispa danica. Park.

Ocymum latifolium maculatum, vel crispum. C. B?

Opor in Tanzceto, Menthâ, Ocymo, Matricuriâ augetur cum crispaturâ, quod singulare.

Bullat八 folia $\S \cdot 374$. fiunt plerumque ex rugosis $8_{3}: 6_{4}$. auctâ , et multiplica! $\hat{a}$, adeoque elevatâ substantiâ folii intra ejusdem vasa:

Ocymuin foliis bullatis. C. B.

Brassica undulata. Rerealm.

Lactuca capitata foliis magis rugosis. $\mathrm{B}$.

Lactuca capitata major, foliis rugosis, et contortis. B. Lactica capitata omnium maxima verrucosa. $\mathrm{B}$.

SAPONARIA concava anglica, ob florem variantem

(p. 83.) lepidè Gentiana folio convoluto J. B. dicta, singulari modo bullatum folium constituit deficientibus rugis; coarctato enim margine concava evadunt folia cochlearis instar.

Tenuifolize. Plantæ interdum nascuntur ex latifoliis, sed minùs frequens est hrec varietas.

Heracleum hirsutum, foliis angustioribus. C. B.

Lycopus foliis in profundas lacinias incisis. T.

Prassica angusto apii folio. C. B.

Veronica austriaca, foliis tenuissimè laciniatis. T. Sambucus laciniato folio. C. B. 
Sonchus asper laciniatus.' C. B.'

Valeriana sylvestris, foliis tenuissine divisis. C. B.

312. Morbosas plantas, vẹl etiam ætates in Nominibus varietatum assumere, sæpius superfluum est. Morbosæ plantæ a Botanicis receptæ $\nabla$ ariæ suilt, prout earum morbi.

Erysiphe Th. est Mucor albus, capitulis fuscis sessilibus, quo folia asperguntur, frequens in Humulo, Lamio Fl. suec. 494. Galeopside Fl. suec. 49 I. Lithospermá Fl. suec. 152. Acere Fl. suec. 303 .

Rubigo est pulvis ferrugineus, foliis subtus adspersus, frequens in Alchemilla Fl. suec. I 35. Rubo saxarilt Fl. suec. 4 I I. Esula degènere R. et præsertim in Senecione, sive facobera senecionis folio incano perenni. Hall. jen. 177. inprimis in solo sylvatico ustulato.

Clavus, cùm Semina enascuntur in cornicula majora extus nigra, ut in Secali, et Caricibus.

Ustilago, cùm fructus loco seminum farinam nigram proferunt.

Ustilago Horlei C. B. Ustilago Avene. C. B.

Scorzonera pulverifiora H. K. P. Tragopogon abortivum Los.

Nidus insectorum causatus ab insectis, qux ova deposuere in plantis, unde excrescentixe varix.

Gallæ Querci, Glechome, Cisti, Populi tremule, Salicum, Hieracii myophori.

Bedeguar Rosce.

Folliculi Pistacice, Populi nigra.

Contorsiones Cerastii, Veronice, Loti.

Squamationes Abietis, Salicis rosea.

Insecta sæpe causant plenitudines, et prolificationes florum.

Matricaria Chamamelum vulgare Fl. suec. 702. ab insectis minimis fit prolifera.

Carduus caule crispo Fl. suec. 658. curâ insectorum gerit flosculos inajores, griseos plenos, vel potius prolifero-frondosos, pistillis in folia enascentibus.

313. Color facillimè variat, præsertim ex cæruleo, rubrove in album.

CoLORES inprimis sequentes a Botanicis numerantur. Hyalinus, Aqueus, Vitreus. 
Albus, Lacteus, Niveus.

Cinereus, Incanus, Lividus, Plumbeus.

Niger, Pullus.

Fuscus, Luridus.

Ater, Piceus.

Luteus

Flavus, Sulphureus.

Fulvus, Croceus, Flammeus.

Gilvus, Testaceus, Ferrugineus.

Ruber, Sanguineus.

Incarnatus,

Coccineus, Puniceus.

Purpureus, Phceniceus.

Violaceus, Caruleo-Purpureus.

Cæruleus.

Viridis, Prasinus.

Plantarum Colores varii variis partibus magis proprii sunt:

Niger frequenter in Radice, sæpe in Seminibus, rariùs in Pericarpio, rarisime in Corolla.

$V i r i d i s$ proprius Herber, et Calyci; rarissimus in $\mathrm{Co}-$ rollâ.

Hyalinus frequens in Filamentis, et Pistillo.

Luteus frequets in Antberis, nec infrequens in Corollis præsertim autumnalibus, et Semiflosculosis $\mathrm{T}$. Albus frequens in Coroilis vernalibus, et Baccis dulcibus. Ruber frequens in gloribus astivis, et baccis in umbrâ natis, acidisque.

Coruleus nec infrequens in foribus.

FLORUM colores sæpius mutantur.

Ruber in album:

Erica, Serpillum, Eetonica, Galeopsis, Pedicularis.

Dianthus, Silene, Cuiubalus, Agrostemnia, Coronuriz.

Trifolium, Orchis, Digitalis.

Carduus, Serratula, Gnaphalium.

Rosa, Papaver.

Fumaria, Geranium.

Cæruleus in Album.

Campanula, Polenooniuin, Convolvulus.

Hepatica, Aquilegia, Vio!a.

Vicia, Galega, Polygala.

Esbium, Ancbusa, Symphytum, Borrago. 
Hyssopus, Dracocephalum, Scutellarix.

Scabiosa, Fasione, Cyanus, Cichorium.

Luteus in Album:

Melilotus, Agrimonia, Verbascum.

Tulipa, Blattaria, Alcea.

Cyanus turcicus, Chrysanthemum.

Albus in Purpureum:

Oxalis, Datura.

Pisum, Eellis.

Cæruleus in Luteum:

Commelina, Crocus.

Ruber in Cæruleum:

Anagallis.

Multiplex mutatio.

Aquilegia cærulea in rubrum, a'bum.

Pelygala cxrulea in rubrum, album.

Hepatica cærulea in rubrum, album.

Cyanus cæruleus in rubrum, album.

Mirabilis rubra in luteum, album.

Impatiens lutea in rubrum, album.

Tulipa lutea in rubrum, album.

Antbyllis lutea in rubrum, album.

Primula rubra in luteum, album.

Cheiranthus lutea in cxeruleum, album.

Pericarpia Baccata sunt primùm viridia, dein rubra, demum albida; matura etiam variant colores, albos, ru=bros, cæruleos, præsertim in Pyris, Prunis, Cerasis.

Solanum guinsense, fructu nigerrimo. B.

Sol.nnum annuum, bxccis luteis. Dill.

Solanum judaicum, baccis aurantiis. Dill.

Rubus vulgaris major, fructu albo. Raj.

Ribes vulgare acidum, albas baccas ferens. J. B.

SEMina, rariùs licèt, colores etiam mutant:

Papaver hortense, nigro semins. C. B.

bortense, semine albo. C. B.

Avena vulgaris, et alba. C. B.

$$
\text { nigra. C. B. }
$$

Phaseolus vulgaris, fructu violaceo. T.

vulgaris fructu ex rubro, et nigro variegato. T.

fructu albo, venis nigris, et lituris distincto. T.

Pisum maximum, fructü nigrâ lineâ maculato. H. R.P. bortense, flore. fructuque variegaie. C. B.

Q 3 
Faba ex rubicundo colore purpurascente.

$\mathrm{R}_{\mathrm{ADIX}}$ rariùs colore variat.
Daucus sativus, radice albâ. T.
Daucus sativus, radice luteâ. $\mathrm{T}$.
Daucus sativus, radice aurantii coloris. T.
Daucus sativus, ralice atro-rubente. T.
Raphanus niger. C. B.

Folia rariùs mutant viridem colorem, uti in Amarantis: interdum tamen maculas assumunt.

Persicaria cum maculis ferrum equinum referentibus. T.

Ranunculus hederaceus, atrâ maculâ notatus.

Orchis palmata palustris maculata. C. B.

Hieracium alpinum maculatum. T.

Lactuca maculosa. C. B.

Herba tota sæpe alienum colorem induit.

Eryngium latifolium planum, caule ex viridi pallescente, fiore albo. T.

Abrotanum cauliculis albicantibus. $\mathrm{T}$.

Artemisia vulgaris major, caule ex viridi albicante. $\mathrm{T}$. Atriplex bortensis rubra. C. B.

Amarantus sylvestris maximus novee angliae, spicis purpureis. T.

Portulaca sativa, foliis flavis. Moris.

Lactuca capitata rubra. B.

3I4. Locus aquosus folia inferiora, montosus autem superiora sæpius findit.

Difformia folia, sive folia diversæ figuræ, in eadem plantâ rariora sunt.

Tithymalus heteropbyllus. Plum. Pluk.alm. i $2 . f .6$.

Rudbeckia foliis inferioribus trilobis, superioribus indivisis. Hort. ups.

Hibiscus foliis inferioribus integris, suferioribus trilobis. Hort. cliff.

Lepidium foliis caulinis pinnato-multifidis, rameis cordatis amplexicaulibus integris. Hort. cliff.

Aquatice plantæ inferiora folia, et submersa reddunt sæpius capillaria:

Ranunculus aquaticus, folio rotundo, et capillaceo. C. B. Sisymbrium foliis simplicibus dentatis serratis. Hort.cliff. Cicuta, Sium, Phellandrium, Oenantbe, \&cc. 
Montane planta folia inferiora magis integra, superiora autem magis dissecta formant, e. gr. Pimpinella, Petroselinum, Anisum, Coriandrum.

Tenuifolice \$. 312. huc spectant.

Hoc confirmat, quòd omnes ex solo sicco efformatæ sunt.

3r5. Planta naturalis ( 157 ) nomine, varietatibus ( $15^{8}$ ) opposito, notari non debet.

Cùm varietates sint superflux in foro Botanico; ne excrescant differentiæ in infinitum, hæc lex servanda erit; nec naturalem plantam opus est distinguere a monstris.

3r6. Cultura, tot Varietatum mater, optima quoque Varietatum examinatrix est.

Horticulturæ mangonium produxit flores plenos, fructus horces, caulium turiones, herbas altiles, capitatas, teneraque olera; $\mathrm{Hæ}$ sibi relictæ in solo macro sylvestrem induunt naturam, et naturalem.

Vidi lecta diu, et multo spectata labore Degenerare tamen: ni vis humana quotannis Maxima queque manu legeret : sic omnia fatis In pejus ruere, ac retro sublapsa referri. Virg. Georg.I. Sic dulcissimæVites fiant acidæ; suavissima Mala acerba, gratissima Pyra austera; mitissima Amygdala amara, succosa Persica exsucca; lævissimæ Lactuca spinosæ; pulposi Asparagi lignosi; sa pidissima Cerasa acidissima; Cerealia Olera, et Fructus evilescant omnia. Solum mutat plantas, unde varietates enascuntur, et, mutato eodem, redeunt.

Buxus arborescens. C. B. Buxus bumilis. Dod. Xanthium Dod. Xanthium canadense majus. T. Acanthus mollis C. B. Acanthus aculeatus. C. B. Cinara aculeata C. B. et non aculeata C. B. Brunella Dod. Brunella caruleo magno fore. C. B. Myosotis foliis hirsutis H. C. et foliis glabris H. C. Crista galli femina. I. B. et mas I. B.

Cerinthe flore ex rubro purpurascente C. B. et flawo flore, asperior. C. B.

Q4 Va- 
3r7. Varietates diversas sub suà specie colligere, non minoris est, quàm species sub suo genere collocare.

Veterum constantiam in speciebus distinctè tradendis ricit Recentiorum studium numerum plantarum augendi sub fine præcedentis seculi, et infecit scientiam varietatum introductione, loco specierum, dum ob notam levidensem nova species crearetur in ditrimentum Botanices; eò usque processit opinio, ut varietates evaderent Species, et Species Genera; huic Hæresi sese opposuit primus Vaillantius, dein Ego, mox Jussiæus, Hallerus, Royenus, Gronovius, aliique non pauci, ne rieręt scientia.

Pleræque varietates facillimè explicantur, et reducuntur ex collatione notarum variabilium varietatis cum naturali plantâ; Dantur tamen varietatis non paucæ, quæ et ingenium, et experientiam exposcunt.

HELEeborus aconiti folio, flore globoso crocco. Amm. ruth. I0I. Trollius bumilis flore patulo. Buxb. cent. I. p. I5. t. 22. Varietas Hellebori Trollii Fl. suec. 474. nectariis longitudine corollæ.

GENTIANA corollâ bypocrateriformi: tubo villis clauso, calycis foliis alternis majoribus. Fl. lapp. 94. varietas Gentianæe fauce barbatâ. Fl. suec. 203. Alore quadrifido, et calycinis laciniis alternis deplo latioribus.

Funaria bulíosa ratice cavâ, et non cavâ, major, et minor, ejusdem speciei esse docent Perianthium minutissimum, species congener's, squama gemma, foliorum structura, rami situs, bracteæ locus, corolla, siliqua, semina, stigma, sed variat bracteis divisis, et radice magis, minùsque cavâ.

VALIRiana arvensis procox bumilis, semine compresso. T. $V$ aleriana arvensis precos bumilis, foliis serratis. T. Valerizna arvensis serotina altior, semine turgidiore. Mor. Valeriana semine umbilicato nudo rotundo. Moris. $V$ aleriana semine umbilicato nudo oblongo. Moris. Valerianella semine umbilicato birsuto majori. Moris. Valerianella semine umbilicato hirsuto minori. Moris. Valerianella cretica, fructu vesicario. Tournef. cor. Valerianalla semine stellato. C. B. 


\section{VAR I E T A I E S.}

Has fructu diversissimas, foliis sæpius magis incisas, ejusdem speciei esse, evincit caulis dichotomus, radix annua, structura foliorum, coroliz, seminis.

Scorpiurus H. C. in distinctis individuis legumine diversissimo, tamen eadem.

Scorpioides siliquâ campoide bispidâ. I. B.

Scorpioides siliquâ cochleatâ, et striatâ ulyssiponensis $T$. Scorpioides bupleuri folio, siliquis lavibus. Park. Scorpioides siliquâ crassâ. Boëlii Germ.

MEDICAGo leguminibus cocbleatis, stipulis dentatis, caule diffuso. H. C.

scutellata, coronata, birsuta, polycarpos. orbiculata, doliata, lupulina, dicarpos. echinata, ciliaris, spinosa, arabica. turbinata, tornata, rugosa, cretica. tam polymorfha fructu in distinctis varietatibus, quàm cochleæ ipsæ, quas in hisce imitatur natura ad maris littora.

Finem ludentis polymorphæ naturæ vix attingat Botanicus, qui in varietatibus sese exercere velit. 


\section{S Y N O N Y M A.}

318. Synonyma sunt diversa Phytologorum (6) nomina, eidem plantæ imposita, eaque Generica (VII), Specifica (VIII), et V ariantia (IX).

Patres $\$ .9$. conveniebant plerumque in plantarum nominibus, genericis solùm contenti.

Commentatores \$. I0. ob defectum Descriptionum, et Figurarum in Patrum scriptis, variè eorum nomina plantis aplicabant.

Descriptores $\$$. II. I 2. detectis numerosioribus plantis, pro arbitrio nomina imponebant.

C. Baubinus quadraginta annorum labore, in Pinace 1622 . antecessorum nomina conjungebat, et reduxit ad 6000. Species.

Curiosi $\$$. 14 . conquirentes undique novas plantas, reddidere numerum earum duplo auctiorem.

Systematici $\$ .53$. in Generum constructione olim maximè dissentientes, secundùm falsa genera, falsissima nomina plantis imponebant.

Legibus differentiarum specificarum non datis, Botanici Differentias partim triviales, partim variabiles, omnes lubricas, speciebus imponebant.

G. Sherardus, Anglis, Botanicus insignis, Bauhini Pinacem continuare allaboravit, sed fato præventus 1728 . reliquit opus Dillenio.

Dillenius, professor Sherardinus Oxonii, continuavit opus

Sherardi in annum, quo obiit, 1747 .

Sibthorpius, Successor Dillenii, Pinacem Sherardianum, et Dillenianum, etiamnum ineditum, servat, et supplet. Hallerus variis in Operibus Synonymiam absolutam plantarum Helveticarum elaboravit.

Synonymice absolutæ opus maximè necessarium est Botanicis:

Unico auctoris nomine detecto, innotescunt omnium. Evolvi ideoque possunt Descriptiones, et Figuræ 0mnes. Innotescunt inde omnia, quæ beneficio seculi innotuere de plantâ.

(rum.

Plura nomina non reddant amplius ideam plurium plantaSpecierum synonyma ad Botanicos præcipue spectant; $V a-$ rietatum autem, qux sæpius superfua, addat qui velit, ut minuatur specierum falsarum numerus. 
319. In Synonymis nomen optimum agmen ducat, quale sit nomen alîs Selectum, aut Auctoris proprium.

Inter Synonyma primum erit Auctoris, sive hoc proprium, sive $a b$ alio desumtum.

Primum Nomen Speciei erit selectum, et inter Synonyma prestantissimum.

Malè itaque in fine Synonymorum Auctores nonnulli proprium nomen collocant.

Alsine minor Fucbs.Lon.Tab. minor.Rudb. miror multicaulis C. B. Morsus gallinæ minor Brunsf. Spec. 4. Trag. ALSINE vulgatissima Nostra Brom. chlor.

Differentias genuinas in nomine receptas alii nominibus specificis vagis, et illegitimis postponunt:

Veronica mas supina vulgatissima: C. B. Seguier.veron.

I.p. 233. Berg: viadr. 7 6.Ludolf. berol. 2 I 2. Veronica supina vilgaris. Moris. Veronica mas serpens. Dod. VERONICA caule repente, scapis spicatis, foliis ovatis strigosis. Lin. chiff. 8. VEROINICA, foliis siccis ovatis serratis, caule procumbente, ex alis ramosa. Haller. belv. 530.

320. Synonyma eadem conjungantur.

Dupliciter Synonyma proponuntur, vel descendendo ab antiquissimis ad nova, vel ascendendo a novis ad prisca.

Descendendo Inventoris nomen aciem ducat, ubi synonyma secundùm Chronologiam proponuntur ; quam qui elaborare studet, retro ui incedat, necesse est: vide Hallerum, Dillenium.

Ascendendo nomina proponunt, qui incipiunt a nominibus. genericis recentioribus, desinentes in antiquissima, quod mihi sæpius receptum.

Adscendentia.
LIMOSELLA. Fl. lapp. 249. LIMOSELLA. Fl. lapp. 249. Fl. su. 521 . Hall. belv. 609. Fl. su.521. Hall. belv. 609. Wach.ultr. 1 44.Gusit. stamp. W'ach.ultr. I44.Guett.stainp. 1. p. 195. Dulib. paris.193. 1. p. 195. Dalib. paris. 193. Limosella annua. Lind. alsat. Plantago aquatica minima. 156. t. 1. Clus. bist. 2. p. 110. Park. Menyanthoides vulgaris.Vaill. theutr. 1244 . Mlerret. pin.95. par. 126. Fabreg. V. p. .91. Boerlo lugid. 1. p. 45 . 
Plautaginella. Rupp. jon.I 8. Plantaginella palustris. Bauh. Dill.gen. I I 3. Euxb. act. 3. pin.190. MI gris.bist.3.p.605. p. $27 \mathrm{I}$.

Plantaginella pa!ustris. Baub. pin. 190. Moris. hist. 3. p. $605 . f$. I 5. $t .20 . f_{i}$ I. Raj. Sp:rgula perpusilla lanceola is angl.237. hist. 1077. syn. 3 . p. 278 . Mapp. alsat. 242 .

Platutago aquatica minima. Clus. hist. 2. p. I lo. Park. theatr. I 244 . Merret.pin. 95. Boerh. lugdb. 1. p. 45 .

Spergula perpusilla, lanceolatis foliis. Loes. pruss. 20 I. $t .8 \mathrm{I}$.

Alsine palustris repens, foliis lanceolatis, floribus albis - perexiguis. Pluk. alm. 20.t. -74.f. 4. Volk. norib. 22.

Alsine palustris exigua, foliis - lanceolatis plantaginellæ aquatica instar; flosculis albis vix conspicuis rienir. pag.2. t. 7.f. 6. Comm: boll.8. Raj. supplem. 498 . Tournef. paris. 38.

Panunculus palustris minimus - plantaginis folio. Herm. lugdb. 5.17. foliis. Loes. pruss. $26 \mathrm{I} . t .8 \mathrm{r}$. Alsine palustris exigua, foliis lanceolatis plantaginella acuaticre instar, flosculis albis vix conspicuis Mentz. pag.2.t.7.f:6. Comm.boll.8. Raj. supplem. 498. Tournef. paris. 38 I.

Alsine pulustris repens, foliis lanceolatis, floribus albis pærexiguis. Pluk. alm. 20. t. 74.f. 4. Volk. norib. 22.

Ranunculus palustris minimus, Plantaginis folio. Herm. lugab. 5 I 7 .

Plantaginella. Rupp.jen. 18. Dill. gen. I1 3 . Buxb. act. 3 . p. $27 \mathrm{r}$.

Menyanthoides vulgaris $V$ aill. puris. I 26 . Fabregouv Vaill. p.ris. 9 I.

Limosella annua. Lind. alsat. I 56. $t$. I.

$32 \mathrm{r}$; Synoyma singula novam ordiantur lineam. Methodo quintuplici solent Synonyma ab Auctoribus recitari.

a. Synonyma secundim genera.

PARTHENIUM foliis ovatis crenatis. Hort. cliff. 442. Gron. vire. I 15. Roy. lugdb. 86.

Partheniastrum, helenii folio. Dill. elth. $302 . t .225 \cdot f .292$. Ptarmica virginiana, folis helenii. Moris. bles. 207.

Ptarmica virginjana, scabiosæ austriacx foliis dissectis. Pluk. alm. 308.t. 53.f.5. et t. 2 I9. f. 1.

Pseudo-Costus virginiana,s. Anonymos corymbifera virginiana, flore albo. Raj. bist. $36_{3}$. 


\section{S Y NON Y MA.}

Dracunculus latifolius,s. Ptarmica virginiana, folio helenii. Moris. hist. 3. p. $4 \mathrm{I}$.

b. Synonyma per Lineas innovatas.

PARTHENIUM foliis ovatis crenatis.. Hort. cliff. 442. Gron. virg. I I 5. Roy. lugdb. 86.

Partheniastrum helenii felio. Dill. elth. $302 . t .225 \cdot f \cdot 202$. Ptarmica virginiana, foliis helenii. Moris. bles. 297.

- - - - scabiosæ austriacæ foliis dissectis. Piuk. alm. 308.t. 53.f. 5. et t. 2 I 9.f. I.

Pseudo-Costus virginiana, s. Anonymos corymbifera virgiriana, flore albo. Raj. hist. 363 .

Dracunculus latifolius, s. Ptarmica virginiana, folio helenii. Moris. kist. $3 \cdot p \cdot 4 \mathrm{I}$.

c. Synonyma per seriem continuatam.

PARTHENIUM foliis ovatis crenatis. Hort. cliff. 442. Gron. virg. I I 5. Roy. lugdb. 86. Partheniast rum helenii folia. Dill. elth. 302. t.225. f. 292. Ptarmicavirginia. na, foliis helenii. Moris. bles. 297 . Ptarmica virginiana, scabiosæ austriacæe foliis dessectis. Pluk. alm. 308 . t. 53 . f. 5. et t. 2 I 9. f. I. Pseudo-Costus virginiara, s. Anonymos corymbifera virginiana, flore albo. Raj. bist. 363 . Dracunculus latifolius, s.Ptarmica virgiciana, folio helenii. Moris. bist. $3 \cdot p \cdot 4 \mathrm{I}$.

d. Synonyma per exclusionem nominis generici insequentis. PARTHENIUM foliis avatis crenatis. Hort. cliff: 442 . Gron. virg. I I 5. Roy. lugdb. 86. Partheniastrum helenii folio. Dill. elth. 302.t.225.f. 292.Ptarmica virginiana, foliis helenii. Moris. bles. 297. virginiana, scabiosae austriacæ fuliis dissectis. Pluk, alm. $308 . t .53 \cdot f$. 5 . ct t. 2 I 9. $f$. I. Pseudo-Costus virginiana, s. Anonymos corymbifera virginiana, fore albo. Raj. bist: 363 . Dracunculus latifolius, s. Ptarmica virginiana, fotio helenii. Moris. hist. 3.p. 41 .

e. Synonyma per abbreviationem cum parenthesi.

PARTHENIUM fcliis ovatis crenatis. Hort. cliff. 442. Gron. virg. 1 5. Roy. lugatb. 86. Partheniastrum helenii folio. Dill. elth. 302 t t. $225 \cdot f .292$. Dracuriculus latifolius (s. Ptarmici virginisna (scabiosæ austriacæe foliis dissectis. Pluk. alm. 208.t. 53.f. 5. et t. 2 I g. f. 1.) folio helenii. Moris. bles. 297.) hist. $3 \cdot p \cdot 4 r$. Pseudo-Costus virginiana, s. Anonymos corymbifera virginiana, flore albo. Raj. hist. 363 . 
Receptum est ( $a)$ a me; (b) a Mentzel. lex.; $(c)$ a plerisque; $(d)$ a Dill. giss.; (e) a Brom. chlor.

322. In Synonymis Auctor, et Pagina ubique ad finem indicanda sunt.

Citatio operis in nominibus plantarum constabit genere, et differentiâ, s. Auctore, et Libro.

Auctoris nomen solum non sufficit, cùm idem aucior sæpius plura scripta evulgavit; F'uere etiam sæpius duo, vel plures ejusdem nominis, vel in posterum possent plures prodire eodem nomine inclarescentes; Sic dudum innotuere duo ejusdem nominis:

Gesneri. Ammanni. Rudbeckii.

Baubini. Knautii. Commelini.

Camerarii. Volkamerii. Magnolii. Hermani. Horstii. Triumfetti. Jussicei. Mylleri. Montii. Scheucbzerii. Cordii. Besleri. Hoffmanni.

Opus solum pro citatione non sufficit, cùm plurima, vel prodiere dudum, vel prodibunt in posterum sub eodem nomine, quæ ansam confusionis præbent futuris seculis, nisi Auctor addatur.

Hort. Lugdb. Hort. Patav. Flor. Parisin.

$\begin{array}{lll}\text { Vorstii } & \text { Cortusi } & \text { Cornuti } \\ \text { Pavii } & \text { Guilandini } & \text { Tournzfortii } \\ \text { Scbyylii } & \text { Schenckii } & \text { Vaillantii } \\ \text { Hermanni } & \text { Veslingii } & \text { Fabregorvii } \\ \text { Boerbaavii } & \text { Marcelli } & \text { Dalibardi. } \\ \text { Royeni. } & \text { Turrei. } & \end{array}$

Proprii Auctores solent excludere proprium nomen, et tantùm operis mentionem injicere, vel quod minus placet, etiam tantùm ejusdem literas initiales; hoc $a b$ aliis non erit imitandum:
(Dill.) Catal. Giss. vel C. G. Hort. Eltham. .. H. E. Hist. Muscor. . . H. M.

Compendiosissimè, et unico vocabulo nominetur opus, cujus litera initialis erit parva, Auctoris verò majuscula, ne citatio evadat diffusior, tamen evidens.

Pagina addatur ultimo, ut faciliùs evolvatur planta. 


\section{SY NON Y M A.}

323. In completầ Synonymorum cohorte Inventoren Asterisco notare placet.

Difficile hoc fit, antequam nova Synonymia, et absoluta prodeat.

Multum lucis adferret ejusmodi nota in Chronologiâ plantarum, ne quæratur apud antiquos a recentioribus detecta planta, præsertim si Synonyma collocentur secundùm genera, $(\$ .32 \mathrm{I}$.)

324. Nomina Regionum vernacula vel excludenda, vel ad Finem Synonymorum collocanda sunt.

Nomina cujusvis loci vernacula in Floris specialibus multum lucis accendunt, non modò ut faciliùs ab incolis addiscantur plantæ, sed et ut ex vulgi nomine, sæpius ingenioso, natura plantæe innotescat.

Barbara nomina ad finem Synonymorum collocentur, uti

Rheedi Malabarica, Hermanni Zeylanensia, KeMpFeri faponica, Hernandi Mexicana, Marcgravis Brasiliensia, RUMPFI Amboinensia. 


\section{A D U M B R A T O NES.}

325. Adumbrationes Historiam plantæ continent, uti Nomina (VII), Etymologicis (234-242), Classos (II), Characteres (VI), Differentias (VIII), Varitates (IX), Synonyma (X), Descriptiones (326), Icones (332), Loca (334), Tempora (335).

Metbodus demonstrandi proposita in Syst. Nat. 6. p. 222. sistit ordinem, secundùm quem plantæ historia concinnari debet.

Continebit Adumbratio omnia, qux ad Historiam plantee pertinent, uti ejusdem Nomina, Signa, Faciem, $\mathrm{Na-}$ turam, et Usum.

326. Descriptio ( 325$)$ est totius plantæ character naturalis, qui describat omnes ejusdem partes externas (80.81.82.83.84. 85. 86.)

Perfecta Descriptio non adquiescat more recepto in Radice, Caule, Foliis, et Fructificatione, sed etiam probè observabit Petiolos, Pedunculos, Stipulas, Bracteas, Glandulas, Pilos, Gemmas, Foliationem, et Habitum omnem.

RICINUS foliis peltatis serratis, petiolis glanduliferis. Hort. cliff. 450.

Radix Ramosa, Fibrosa.

Caulis erectus, teres, viridis, articulatus, inanis, levis: striis sparsis longitudinalibus, superni fexuosus, altus orgyam unam, alteramve.

Rami solitarii, ex axillis superioribus foliortum, cauli similes, altiores; ex inferioribus axillis breviores, vel marcescentes, vel seriores Rami.

Folia alterna, peltata, novemlobata: Lobis exteriorilus najoribus, magis angulatis: Nervis totidem ab umüilico all loborum apices excurrentibus : obtusiusculè incequaliter serrata, reticulato-tenosa, utrinque levia, supra glabra, disco extrorsum versa.

Hæc ante explicationem plicata, serraturis glandulosis. 
Petioli teretes, leves, patentes, sessiles, filiformes, longitudine foliorum.

Glandula suprabasim petioli, latere superiori,obtusa, solitaria. Glandulæ bine peltate in apice petioli: latere superiori.

Glandulæ duce opposite, ad basim petioli, in caule.

Stipula petiolo opposita, membranacea, glabra, solitaria, caulem ambiens ad petislum usque, concava, acuta, decidua.

Pedunculus ramos terminans, e regione petioli inter ramum, et stipulam, erectus, nulus, adspersus, Umbellulis alternis, sessilibus.

Involucrum umbellule tripbyllum, membranaceum, minimum, incequale, marcescens.

Umbellulæ inferiores multiflora, masculce; superiores pauciores, uniflore, feminece.

Pedicelli alternatim excrescentes, deforentesque.

Flores Masculi pedicellis longioribus insidentes.

Masculi. Calycis Perianthium monopbyllum, quinquepartitum : laciniis ovatis, concavis. Corolla nuila. Staminum Filamenta varia, filiformia, rumosa, et subramosa, calyce longiora. Antheræ subrotunde, didyma. Feminei. Calycis Perianthium monopbyllum, tripartitum, deciduum : laciniis ovatis, concavis. Corolla nulla. Pistilli Germen ovxtum, tectum spinis subulatis inernibus. Styli tres, bipartiti, erecio-patentes, subulati, bispidi, purpurascentes. Stigmata simplicia. Capsula subrotunda, trisulca, obsoletè triangularis, unilique aculeata, trilocularis, trifariam debiscens, elastica. Semina solitaria, subovata, macuitis incequalibus.

327. Descriptio $(326)$ compendiosissimè, tamen perfectè, terminis tantùm artis, si sufficientes sint, partes depingat secundùm Numerum, Figuram, Proportionem, Situm.

Character Naturalis speciei eodem modo, quo generis(167), confici debet, sed admittat notas plures accidentales, quàm Generis character.

Notæ characteristicæ Descriptionis primariæ semper observandx in omni parte plantæ; sunt $a$ ) Numerus, $b$ ) Figura, c) Proportio, d) Situs. 
PHARNACEUM glabrum, pedunculis folia æquantibus. Alsine Amin. ruth. 84. Rostocbii frequens.

Radix $a$. unica, $b$. filiformis, subramosa. c. albida. d: perpendicularis.

Caules $a$. plures, $b$. filiformes, teretes, glabri, geniculati: articulis nudis, $c$. digitales, $d$. erectiusculi, demum procumbentes, $e$. incarnato-colorati, pellucidi.

Rami a. rarissimi, $b$. facie cuulis, c. caule altiores, $d$. ad genicula enati.

Folia radicalia a. numerosa, b. linearia, integerrima, avenia, succulenta, subtus convexa, margine obtusiuscula, apice subacuminata. c. longitudine sopius articulorum caulis, d. inferiora sensim breviora.

Caulina Folia d. verticillata, a. terna, quaterna, plurave, b. radicalibus similima, figurâ, et $c$. magnitudine.

Pedunculi a solitarii, rariùs bini, $b$. fliformes, co articulis paulo breviores, angustioresque, d. e geniculo inter folia enati, desinentes apice Pedicellis a. duobur, tribusve, b. capillaribus, budis, apice uniforis c. incequaliter proleuntibus, florentibusque, $\bar{d}$. basi articulatis, absolutâque fiorescentiâ depententibus.

Bracteæ a. numero padunculorum, $b$. ovate, $c$. minime, $d$. ad basim pedunculi communis, et partialium.

Florum Calyx Perianthium $a$. pentapbyllum $: b$. foliolis subovatis, concavis, $c$. cequalibus, d. basi levissimè unitis. Corolla nulla, vel calyce unita (cim Periantbium sub florescentia margine, et interiore latere niveum). Staminum filamenta a. quinque, $b$. subulatx, c. longitudine calycis, d. receptaculo inserta. Antheræ a. solitarice, b. basi bifi$d \propto, c$. brevissime, d. divisurâ annexce. Pistilli Germen a. unicum, b. ovatum, trigonum. c. brevissimum. Styli $a$. tres, $b$. fliformes, c. longitudine staminum. Stigmata b. obtusa. Pericarpii Capsula b. ovata, obsoletè trigona, c. longitudine calycis, d. calyce tecta, trilocularis, trivalvis. Semina a. numerosa, b. orliculata, depressa, margine acuto cincta, nitida, c. paria, d. columelle affixa.

328. Descriptio ordinem nascendi sequatur.

Ordo Descriptionis secundùm ordinem partium plantæe incedat.

Latitudinem interdum admittit hæc regula, sed non omnimodam. 


\section{ADUMBRATIONES.}

Malè primùm cirrhi, dein pedunculi, tum glandulre, demum folia, tandem caulis, ultimò petioli promiscuè proponerentur.

Præstat naturam sequi a Radice ad Caulem, Petiolos, Folia, Pedunculos, Flores.

TILIA Hort. cliff. 204.

Radix: Caudex descendens divuricatus, ramosissimus, teres, flexuosus, epidermide deciduâ: Radiculis capillaribus feexuosis subramosis.

Caudex adscendens arboreus, teres, ramosissimus, cortice crasso, poroso, tectus Epidermide in antiquo stri.tto-rimosâ, in tenello levi , glabrâ, ramis præcedentis anni instructis gemmis alternis.

Gemmæ ovatre, prominentes, constructe Squamis duabus, alternis, oblongo-ovatis, obtusis, convolutis, subcarnosis, stipulaceis.

Stipulæ geminacere opposite, ovate, glabre, integerrime, concaver, involventes folia, et caulem.

Caulis simplicissimus, teres, subfexuosus a folio al folium, decunbenti-patens, levis, alspersus punctis aliquat vagis obsolatis.

Folia tenella conduplicata, secunda, rugosa, villosa undique; adulta cordata, nervosa, venosa, inequaliter serrata, acuta, supra glabra, pilis vix conspicuis adspersa, subtus in axillis vasorum majorum barbata.

Petioli teretiusculi, leves, folio breviores, ferè distichè prodeuntes, interjectis spatiis folio brevioribus.

Pedunculi solitarii, petiols laterales, longiores, filiformes. apice trifidi: lateralibus trifidis, extremitatibus uniforis; Flos:uli adeoque septem, alitudine requales.

Bractea lancenlata, obtusiusculx, albo-colorata, integerrima, longitudine pedunculi, a basi ad medium longitudinis pedunculo unita.

Floris Perianthium quinquepartitum, concavum, colorato-flavescens, magnitudine ferme corollee, decilium. Corollie Petala quinque, oblonga, obtusa, apice crenata, flavo-alo bicantia. Staminum Filamenta plurima: triginta, vel quisdraginta, setacea, receptaculo inserta, longitistine corolle. Antheræ subrotunde. Pistilli Germen suts-bbosum, hirsutum. Stylus cylindricus, altituline staminum. Sigma obbusum, pentagonum. Pericarpium coriaceusn, globosum, $\mathrm{R}_{2}$ quin- 
quinqueloculare, quinquezalve, basi debiscens. Semina solitaria, subrciund.l.

Cotyledones quinquefidi, extremis, et mediâ longioribus. I. B. 329. Descriptio distinctas partes plantarum in distinctis paragraphis tradat.

Partes plantarum in descriptione æquè distinctæ evadant, ac in ipsâ plantâ.

Majusculis literis pingantur omnes Partes plantæ, at partium partes minusculis, et a vulgaribus diversis.

Usus inde resultat, quod non modo partes a Lectore faciliùs reperiantur, sed et quod omissa faciliùs in descriptione perspiciantur.

Tædiosius nihil descriptione amplissimâ, quam nec paragraphi, nec majusculæ literæ, secundùm partes plantx , distinguunt.

PASSIFLORA folits trilobis cordatis pilosis, involucris multifido-capillaribus. Amœn. academ. 228.

Radiz fibroso, anizua.

Caulis teres, pirus quam bumane altitudinis, debilis: Pilis $p x-$ tentissimis, inequalibus, albis adspersus. Rami supra axillas spatio remoti, cau!em structurâ imitantes.

Folia alternd, remotz spatiis longitudine foliorum, cordata, obsoletè triloba, basi quinquenervia, venosa: venis diaphanis, iniegerrima, obscurè ciliata: Setis glanduliferis; $P$ ilis erectis, albis, uirinque adspersa; ante explicationem triplicata.

Petioli teretes, similiter pilosi, patentes, folio dimidio breviores, supra partum planiusculi; glandulis subulatis, alternis, erectis, longitudinaliter dispositis in supinâ parte. Stipulz lunares, amplexantes caulem, persistentes, nuda, ciliate: glandulis subulatis, longitudine stipularum.

Cirrhi filiformes, patentes, asillares, pilosi, supra medietatem spiraliter conzoluti, et revoluti, foliis longiores. Pedunculi axiliares, cirrbo laterales, solitarii, erecti, teretes, peisolo breviores, pilosi, terminati inzolucro.

Involucrum triplyilum, lineare, pinnatifidum, pilosum, cilioturn glandulis subulatis, persistens, uniflorum.

Flos sessilis, calyce pentaphyllo, piloso, mucronato \&c.

330 . Descriptio justò longior, aut brevior, utraque mala est. 
Longa nimis evadit descriptio, cùm Color viridis in Herbâ, Mensura partium; et similia, facillime variantia, diffusâ oratione proponuntur.

Breviores justò evadunt Descriptiones, cùm excluduntur notæ singulares, et partes essentiales Herbæ, licèt minimæ, uti Stipulæ, Bracteæ, Glandulæ, Pili, et similia. Lini descriptio sit exempli loco:

A. Brevissima, et imperfecta Dolonei.

Radices exiles. Culmi, seu virge tenues, rotunds. Folia obionga, angusta, acuminata. Flores in summis cirgis, spzciosi, carulei. Vascula paroa, rotinda, orbiculata. Semen aliquatenus oblongum, leve, glabrum, spiendens, ex fulvo-punicans.

B. Longissima, superflua, et inanis.

Radices angustre, subdivise, intraterram recondite.

Caules erecti, rotundi, virides, bipedales, vel tripedales, ramosi:

$\mathrm{R}$ amis caule dimidio brevioribus.

Folia ang usta, virilia, acuta, plurima, uncialia, ad angulum acutum a c.ule discelentia, busi affixia, non tomentosa, aut villos. ; superior.a folizatantium semiuncialia sunt, et quatuor lineas lata; inferiorat tres lineas lata, at suprema vis duas lineas altingunt in latitudine. Pedunculi simplices, uncizles, vel sesqui unciales, crassitie vix dimidie linere. Flores in sum:nitatibus rannorum; ampli, patsnies, \&c. ut pag. 133 .

C. Naturalis, et legitima descriptio:

Radix simplex, perpendicularis, fiexuosa, pallida : radiculis lateralibus capillaribus.

Caulis simplex, perpenticularis, teres, filiformis.

Folia alterna, sessilia, lanceolata, obsolete trinervia, actiminat.a, erectiuscula, utrinque glabra: sub.axillaria pasu!a miajora.

Rami ex axillis foliorun summorum, erecti, foliis minoribus instructi: rudimenta ramorum inarcescentia ex axillis foliorum inferiorum.

Pedunculi foliis oppositi, eisque longiores, filifor mes, nuki, terminati Fructiticatione solitariâ, in generizus descriptâ.

Cotyledones quasi quatuor, cruciatim oppositi, quorum duo inferiores subovati, superioribus duplo latiores.

331. Mensura Magnitudinis, a manu desumta, in lantis convenientissima est. 
Mensuram plantarum secundùm scalain geometricam, et quidem adcuratissimam, in partibus plantarum describendis, introduxit Tournefortius, quem secuti sunt asseclæ, ut essentia descriptionis consisteret in adcuratissimâ mensurâ geometricâ.

Variare plantas in partium longitudine, et latitudine, magis quàm in re aliâ quacunque,cuique in Botanicis versato esse notissimum nullus dubito.

Admitto rarissimè aliam mensuram, quàm proportionalem inter plantæ partes diversas, ubi pars hæc, vel illa, longior aut brevior, latior aut angustior sit alterâ.

Si verò mensura, vel scala assumenda sit, non judico opus esse, ut ejusmodi scala a Botanico circumferatur, sed ut mensuretur a manu, vel staturâ hominis.

Capillus est diameter Crinis.

Linee pars duodecima.

Linea est Lunulæ longitudo, ab unguis radice versùs unguem extensa (non verò in pollice).

Linea una Mensure parisine.

UNGUTs est longitudo Unguis.

Linere sex, sive vncia dimidia.

Pollex est longitudo, aut diameter extimi articuli Pollicis. Uncia una parisina.

PALMus est diameter quatuor digitorum transversorum parallelorum, demto pollice.

Unci \& tres parisienses.

Dodrans est spatium inter apicem Pollicis, et Digiti minimi extensum.

Uncia norem.

Spitharia est spatium inter apicem Pollicis, et Indicis extensum.

Uncia septem.

PES mensuratur a flexurâ cubiti ad basim Follicis.

Uncice durdecim.

Cubitus a flexurá cubiti ad extimum apicem digiti intermedii.

Uncie septcmidecim.

Rrachium ab axillà ad apicem Digiti intermedii.

Uncia riginti quatuor.

OrgYa, Hexapoda, seu Humana altitudo est mensura inter manus expansas.

Pedes sex. 
332. Icones magnitudine, et situ naturali depingendæ sunt.

Veterum icones sistunt maximas Arbores, et minimas Alsines sub eadem magnitudine; Procumbentes, et Repentes plerumque erectas pingunt, quod sedulò vitandum. Cùm magnitudo nequeat depingi in plantis maximis, præestat Ramulum porrigere, et plantam integram in compendio juxta partem sistere; uti Ebret in Napæa t. 8. Hibisco t. 6. Verbena t. I 4. et Martynia t. $x$.

Fundamentales figuræ $\$$. I I. præsertim Plumerianæ, facillimè elaboratæ, plantas optimè repræesentant.

Lignere figuræ \$. I I. olim usitatissimæ, præsertim Rudbeckianæ, certabant cum æneis, sed opera Botanica reddidere minus pretiosa; nunc antiquatæe sunt non sine impensis Botanicis.

Pictor, Sculptor, et Botanicus æquè necessarii sunt ad figuram laudabilem; Si alter horum peccet, evadit figura vitiosa. Hinc Botanici, qui unâ execuere artem et pictoriam, et sculptoriam, præstantissimas figuras reliquerunt.

333. Icones optimæ omnes plantæ partes, licèt minimas etiam fructificationis, exhibeant.

Minimis in partibus, præsertim Fructificationis, latent numerosissimæ, et præstantissimæ Differentiæ, quæ Speciem maximè distinguunt.

Pili, Glandulæ, Stipulie, Stamina, et Pistilla, in veterum figuris exclusa, nunquam in Icone omittenda, si evadet digna.

334. Loca natalia plantarum respiciunt Regionem, Clima, Solum, et Terram.

Horticulture fundamentum dependet a loco plantarum, unde Canones, et Principia artis formanda, quod demonstravi in Actis Stockbolm. 1739. p. I.

Milleri The Gardeners Dictionary tradit specialem culturam singulæ plantæ, at nimis diffusa, et onerosa esset hæc Methodus Horticulturæ per omnes plantarum detectas species.

Habitationes plantarum ex loco patescunt, quibus constat, unde Plantarum species conquirendie pro Herbariis, Hortis, Medicina, et Oeconomiâ.

$$
R_{4} \text { RE- }
$$


REGIO tradat Regnum, Provincias, Pagos, et ubi rarissimæ plantre, loca specialissima.

CLIMA habet tres dimensiones, Latitudinem, Longitudinem, et altitudinem Loci.

Latitudo est ab Equatore vel Borealis, vel Australis, utraque gradibus divisa 90 .

Longitudo communiter ab Insulâ Ferro Canariensium ad eamdem numerat 360 .

Altitudo perpendicularis est terræ mensura a mari, versùs summa cacumina Alpium.

Mensuratur Barometro-secundùm elevationem loci perpendicularis descendente, cujus scala ex mensurà anglicanâ hæc est:

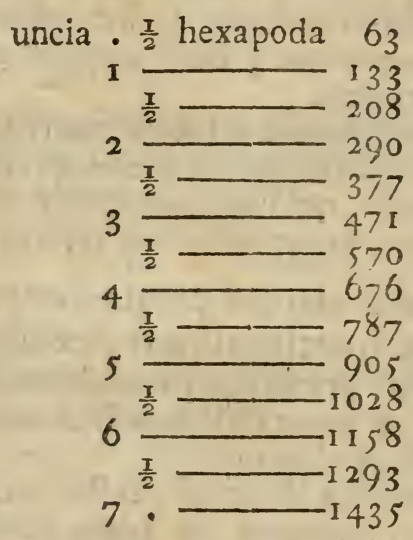

Vaillantius inter primos Climata assumsit in loco natali plantarum, sed unicè latitudinem respexit, e. gr. Panax foliis ternis quinatis sub latitudine 45.46 . Non verò suffi-? cere solam latitudinem, patet ex locis dissitis, diversissimas plantas proferentibus; multò minùs longitudinem. Latitudines Rome Italix, Pechini Chinæe, Noveboraci Americes, sub eodem gradu Boreali ferme sitæ sunt. Roma 4I: 51. Pekin 39: 55. Noveboracum 4I: 0. Similiter Palcestina, et Florida Boreales. Caffria, et Chili Australes.

latitudine ferè conveniunt (Cap. Bon. Sp. 34: I5. Jerusalem $3 \mathrm{I}: 40$ ) ; sed diversissimas plantas alunt. - Longitudine conveniunt Promontorium Septentrionale (NordCap.), Upsalia, Roma, Caput Bon. Spei plantis diversissimis. Al- 


\section{A DU MBRATIONES."}

Altitudo magis conveniens est pro habitatione plantarum. Aquaticæ Plantæ Indicæ cum Europæis sæpe conveniunt, uti Utricularia, Drosera, Aldrovanda, Nymphea, Sagittaria.

Alpince Plantx Lapponiæ, Groenlandix, Sibirix, Helvetix, Wallix, Pyrenxerum, Olympi, Ararat, Brasilix, licèt remotissima, sæpe exdem sunt. Sirata Telluris supra aquam ubique, ni fallor : eadam, et eodem ordine posita sunt: supremum Petra, dein Schistus, tum Marmor, demum Schistus, tandem Cos. It. wgoth. 77 .

Sit pratum mari paulò altius plantis pratensibus uberrimis refertum, elevetur adjacens; magisque a mari remota terra, mox aliæ plantæ, et sylvestres hanc operient, cujus exempla in $I t$. Scan.

SOLUM respicit Telluris naturam:

MARE, aquá salsâ rofertum, occultat plantas radicibus destitutas, per poros nutriendas, frigoris impatientes:
Fucus.
$\Lambda^{\top}$ ajas.
Ulva.
Zostera.
Ceratophyllum.
Spongia.

LitTora maris Arenâ, sive Sabulo, sale imprægnato, maris fluctibus, ventisque exposita, alunt proprias plantas.
$\begin{array}{ll}\text { S Salicornia. Crambe } \\ \text { Salsola } & \text { Beta }\end{array}$
Cakile
Atriplex
Glaux:
Anastatica
Eryingium
Triglochin
Statice
Seripbium
Samolus
Arenaria mar. Ceniaur. minus.
Isatis.
Hippophaë.

Fontes scaturiunt aquâ gelìdà, purissimâ;

Mnium Fl. su. 9I 3. Fonitinalis Angelica.

Montia

Hippuris

Fetrosclinum.

Beccabunga.

Fluvir aquam puram, subfrigidam, motu agitatam rehunt.

Potanogeton Sparganium Ranunculus capill. RIP F Fluviorum, et lacuum hycme sub aquâ reconditx. Pbalaris FI.su.48. Hydrocityle
Lycopus
Scutellaria
Lythrum
Limosell.x
Ranunculus F1.su.459.
Eupatorium. Alisnua Lysimacbia. 
LAcus, aquâ purâ repletæ, fundo consistenti gaudent.
Isoëtes
Lobelia Dortman.
Arundo
Subularia
Spongia
Scirpus
Plantago monanth. Nympheea
Elatine minim.

STAGNa, et Fossæ fundo limoso, et aquâ quietâ sunt repleta.

Chara Typha Cicuta

Stratiotes Butomus Sium

Elatine verticillat. Sagittaria Phellandrium.

Vallisneria Hydrocharis Rumex britann.

Callitriche Nymphoides. Ranunculus 457.

Paludes humo lutosâ laxâ, et aquâ refertæ, æastate siccescunt.
2. Hu Cärex Sceptr. carolin.
Menyanthes
Scheuchzeria.
Myrica
Calla.

Cespitose Paludes, refertæ humo mixtâ sphagno, tectæ tuberibus, cinctæ aquâ limosâ profundâ.
Sphagnum
Scirpus Fl. su. 42.
Ledum
Eriophorum
Tetralix
LichenFl.su. 936.
Rubus Fl. su. 4 I 3.
Andromeda

InUNDATA loca hyeme repleta aquâ, æstate putrida exsiccata, imbribus interdum suffusa.
Peplis
Bidens
Cypripedium
Tamarix
Saccharum.

-. Filago Fl. sú. 676 .

ULIGINosa mihi sunt loca sp̣ongiosa, aquâ putridâ laborantia, colonis invisa, nec segetis, nec foni proventui apta, innotescentia propriis plantis.
Primula F1. su. I62. Pedicularis
Ulmaria

Valeriana Fl. su. 3 I. Antbericum ossifr. Comarum

Cynosurus F1.su. 82. Potentilla frutic. Pinguicula

Aira Fl: su. 71. Vaccinium. 312. Burmannia

Cardamine Fl.su. 562 .

Selinumpalustre.

Alpes, Montes altissimi, intrantes Aëris secundam regionem, Arboribus spoliati, summis cacuminibus nive perenni tectis, convallibus humo cespitosâ repletis.
Dryas Pedicularesalp.
Crocus
Sibbaldia Ranunculi alp.
Soldanella
Diapensia Arbutus Fl.su.340. Betula nana.
Azálea Alchemilla digit.
Silene Fl. su. 368.
Androniedre Rumex Fl.su. 294. Veronica Fl. su. I 3.
Bartsia. 575 .
Viola Fl. su. 720.
$-1.5$
Thalictr.Fl.su.455. GentianaFl.su.201.204. 


\section{ADUMBRATIONES.}

Rupes constant petris, murisve præruptis, aridissimisque. Aira.Fl. suec, 64. Polygonitum Capparis Alpë. Asclepias

Cymbalaria

Mesembryanthemum. Melica. Sedum.

Clinopodium

Origanum.

Montes, et Colles sabulosi, aridi, steriles aquam vix admittunt.
Asperulacynanch. Fasione
Oreoselinum
Curlina
Gnaphalium
Arnica
Cneorum
Vaccinia rubra.
Lithospermum
Veronica maj.
Melampyr. Fi.su. 5 ro.

CAMIPI aprici ventis expositi, sicci, asperi sunt.
Artemisia campestris
Buphthalmum
Gentianelia
Pulsatilla
Aàonis lutea
Dancus
Stellaria
Medicago falcat. Bellis
Thesium
Uva ursi
Locusta
Draba
IMyosotis
Ecbium

SyLVæ umbrosæ terrâ sabulosâ sterili refertæ. Hypnum 872. Pyrole
Linneea
Trientalis

Empetrum

Sibthorpia Pulsatill. sylv. Melampyrum F1. su. 5 I4. Solidago.

Nemora ad radices montium, inter Lucus, humo spongiosâ tecta, umbrosa semper, exhalantia continuò aërem humidiusculum, ventis minimè expositum, Plantas Vernales, frigoris, et caloris impatientes, alunt.
Lathraa
Martynia
Hepatica
Actrea
Dentaria
Ainemone
Asarum
Adoxa
Pulmonaria.
Galanthus
Oxalis
Orobus vern.
Leucojum
Fumaria bullosa
Paris
Lunaria
D.iphne.
Impatiens
Prenanthes
Agrimonoides
Mercurialis
Amomum
CardamineFl.su.56 r. Allium ursin.
- Strutbiopteris
Milium
Epimedium
Chrysosplenium. Alsine F1. 37r.

MelampyrumF1.512. Ranunculus Ficar. Cjolamen. 
$\mathrm{P}_{\mathrm{R} \Lambda \mathrm{TA}}$, Herbis luxuriantia, campis depressis, convallibusque constant.

a. Fertilia

\section{Lotus}

Trifolium rubr.

Scorzonera

Campanula

Millefolium

Rbinanthus b. Exsucca Briza

Lagopus

Hypocbreris

Lilium convall.

Viscaria

Bistorta sobolif.

Linum cathartic. Agrimonia

Latbyrus lut.

\section{Heliantbemum}

Mielampyr. 5 I 3 .

Galium luteum Arnica

Ranunculus acris. c. Humidiuscula Alopecurus F1. 52.

Succisa.

Lychnis palustr.

Geum palustre.

Fritillaria

Tbalictrum Fl. su. 453.

Opulus

Frangula

Dulcamara

Clymenum paris.

Parnassia

Pascua differunt a pratis, quòd steriliora, sicciora, et magis sabulosa.

Tormentilla Eupbrasia Ranunculus lanceol.

Pimpinella Brunella Poa Fl. suec. 78.

Sagina

Arva constant agris requietis:

$\therefore$ Aira Fl. su. 72. Erysimum Fl.su.555. Chamrepithys Aphanes Tribulus

Leontice

Anagallis Scundix

Myosotis Myosurus

Latbyr.tuberosus.

Rumex hastat. Anthemis

Ononis

- Campanula Rap.

Fumaria vulg.

Thlaspi.

AGRI terrâ subactâ lætâ gaudent.

Chrysanthemum seget. Vicia Cracca

Ranunculus echinatus Androsace major

MTelampyr. seget.

Convolvulus

G.aleopsis

Helsine scand.

Sinxpis arvens.

Bromus F1. 84.

Napus

Hypecoum

Lolium annuum

Lycopsis

Conium

Sison

Anethum

Nigella

Psylliume

Agrostemma

Triticum Fl. 105. Adonis.

Cyanus

Delpbinium 
Versure, s. Margines agrorum, tanquam prata stercorata considerantur.

Festuca Fl. suec. 9 I.

Lolium perenne

Ścabiosa

Anchusa

Pomaria

Cichorium.

Cerinthe

Aigopodium

Heliotropium

Charopbyllum

Chelidonium.

Culta in Hortis terra, subacta, mixtâ fertilissimâ, promovet plantas Hortulanis invisas, inter olera luxuriantes.
Urtica annua.
Alsine vulg.
Aparine
Sonchus
Chenopodium.
Euphorbia annua
Lamium.

Fineta ex stercore animalium congesta:

$\begin{array}{lll}\text { Urtica maj. } & \text { Xantbium Ricinus } \\ \text { Persicaria } & \text { Blitum } & \text { Datura. }\end{array}$

Asperugo

Ruderata juxta domos, habitacula, vias, ac plateas.
Hyoscyamus
Absintbium
Solanum
Cynoglossum
Chenopodium 209.
Ballote
Lappula
Planiago
Verbascum
Marrubium
Cardiaca
Caucalis
Polygonum.
Erysimum F1.554.

TERRA: plantis faventes sunt IHumus, Arena, Argilla, et Creta.

Hunus tenuissima est plantarum alimentum primarium, observante Kylbelio.

Plantæ itaque pleræque humo delectantur, uti constat experimento Hortulanorum.

Arena sicca, friabilis, siticulosa est.

a. Arena mobilis. S. N. 2 . Arundo Fl. 102.

Elymus F1. 106.

Carex Fl. 749.

c. Arena vulgaris.

Asparagus

Scleranthus

Peganum

Ornithopus

Ulex

Ceratocarpus b. Arena farinacea. S. N. I.

Erica

Pinus

Iberis. Fl. su. 536 .

d. Arena sabulosa, montosa. Heriniaria

Digitalis:

Acinos

Serpilium

Androsace. Fl. su. I6o.

Radiola.

Argilla tempestate humidâ unctuosa, siccâ autem tempestate indurata est. 
Thlaspi Fl.su.53 I. Medicago Fl.su.62 I.Horminum glutinos. Papaver Rbaas Tragopogon Antbyllis.
Blattoria.

Persicaria amphib.

CRETA in collibus siccissimis, aridissimis: Hippocrepis Verbena Resedavulgaris. Onobrychis Campanul.t. H.cliff.4. Cheiranth. luteus. Trifol. capit. asp.

Dignoscitur sic ex solà inspectione plantarum subjecta terra, et solum.

Potentilla vulgaris, colorem argenteum foliorum induens, indicat in terrâ latentem argillam.

Melainpyrum Fl. s. 5io. inontosa

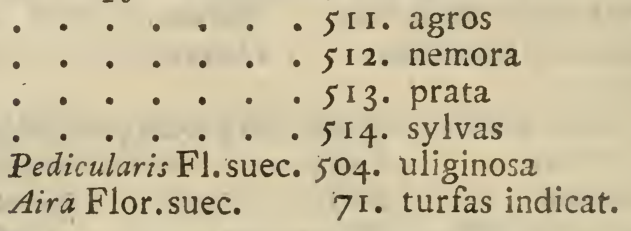

335. Tempus Vigendi, Germinandi, Frondescendi, Efflorescendi, Vigilandi, Fructescendi, Defoliandi indicat Clima.

GERMINATIO est tempus, quo Semina terræ mandata eadem excludunt, in cotyledonum proventum, de quo p. I I 2. Winkler, C. K. Chronica Herbar.

Dieb. 3. Napus, Eruca, Blitum.

4. Anetbum.

5. Lactlica.

6. Raphanus, Cucumis.

7. Hordeum.

8. Atriplex.

15-20. Faba.

19-20. Серx.

40-50. Apium.

Rudbeck. propag. plant. 54 .

Dieb. r. Milium, Triticum.

3. Faba, Sinapis, Rapa.

Spinacbia, Phaseolus.

4. Lactuca.

5. Cucumis, Cucurbita.

Nasturtium. 

6. Beta
10. Brassica.
30. Hyssopus
40. Petroselinim.
Anno I. Persica, Amygdalus. Juglans, Castanea, Peonia.
2. Cornus, Avelluna.

FRONDESCENTIA est tempus æstatis, quo species singulæ plantarum prima folia explicant. e. gr. 1748. Upsalix 1749. Upsaliæe I750. Landscronæ. Apr.28. Ribes F1.195.Apr.29.Ribes 195. Feb.26. Grossularia Maji 4. Palui 396. Maji 2. SyringaH.6.1.Mart.8.Sanbucus. 10. Evonymus. I33. 3. Bitula 776.9 9. Ribes rub. I I. Spirea Hort. I. 4. Padus 396. 10. Pbilaletphus $\begin{array}{lll}\text { 12. Crategus 7. } & \text { 6. Fagus } 785 . & \text { 1 1. Syringa } \\ \text { Populus 819. } & \text { 8. Tilia } 432 . & \text { 12. Rosa }\end{array}$ I 3. Sumbucus i 50. 10. Quercus 784. I 3. Oxyacantha 17. Quercus 784. I 2. Carpinus.786. I 4. Lonicera 18. Fraxinus 830 .

I5. Ulmus

16. Cerasus, Evon.

17. Malus, Alnus

18. Corylus, Opulus

19. Betula, Sorbus

20. Salix viminea

21. Salix caprea

24. Carpinus

25. Salix minor

26. Prunus

27. Populus nigra

28. Pyrus

April. I. Rhamnus cath.

2. Vaccinia nigra.

5. Frangula

7. Esculus

I I. Juglans

12. Tilia

20. Fagus

22. Populus trem.

25. Acer.

Maji. 5. Ouercus

15. Fraxinus 
EFFLORESCENTIA est tempus mensis, quo singula species plantarum primosFlores ostendunt.e.gr. I 748.Upsalix. APRILIS. Fl. succ. MAJI. MAJl. 17. Hepatica 445 . I 5. Draba 526 . 26. Triglochin 299. I8. Fumaria 585. 16. Leontodon 627. 27. Pinus 788. 22. Tussilago 680 . 23. Dapbne 3 I I. 17. Saxifraga 350. Juniperus 824 . 24. Pulmonaria is 6.

25 . Draba 523. 26. Ornitbogalum 270. 19. Chelidonium 430. 27. Viola 7 i6. 28. Pulsatilla 446 . 29. Empetrum 832 . 30. Anemone 450 . IIAJI.

I. Ranunculus 460 .

2. Tussilago 683 .

3. Lathrea 5 I 8 .

4. Murica 817.

5. Viola 7 I 8 .

6. Primula i6 г.

7. Glecboma 483 .

8. Betula 776.

9. Caltha 473 .

ro. Oxalis 385 .

I I. Vaccinium 3 I 3 .

12. Fraxinus 830 .

I3. Viola 719.

14. Androsace 160. Orobus 595 . 18. Adoxa 326.

28. Potentilla 4 I9.

Cynoglossum 154. Hyoscyamus 184. Erysimum 558. 29. Berberis 290. Convallaria 274. Syringa $6: 1$. 20. Fritillaria 8 I: 1.30. Ledium 34 I. Cynosurus 82.

21. Actoa 43 I. Menyanthes 163 . Paris 325.

22. Primula 162.

23. Convallaria 273. Trientalis 302. Crobus 596. Lonicera 192. 24. Pyrus I 30. I. Pyrus I 30. 2. 25. Statice 253. Polysala 586. Loius 6og. Trifolium 6 I 5. Rimunculus 469 . Chrerophylluris 243.
Vaccinium 3 I 2. Asclepias 200. Sorbus 400. Geranium 57 I. Dentaria 565. Ranunculus 472. JUNII.

I. Geum 423. Gnaphalium67 I. Pyrola 334. Thymus 477. Potentilla 4 Is. Bryonia 790. Nymphea 426. 2. Anchusw 153 . \&c. \&c.

Cardui varii non florent, antequam Solstitum absolutum est. Purnıssia foenisecii præco est.

Colcbicum autumni, et gelû nuncia est.

VIGILI E plantarum absolvuntur determinatis horis diei, quibus plantæ flores quotidie aperiunt, expandunt, et claudunt.

SolaresFlores dicuntur,qui determinatum hoc tempus sese explicandi, et claudendi observant ; sunt hi triplices: 1. Meteorici flores solares, qui minùs adcuratè observant horam explicationis, sed priùs, vel seriùs aperiuntur pro ratione umbræ, Aëris humidi, vel sicci, Atmosphæræ pressione majori, aut minori. 
2. Tropici manè aperiuntur, et ante vesperam recluduntur quotidie, sed hora explicationis adscendit, vel descendit, uti dies adcrescit, aut decrescit; adeoque observant horas Turcicas, s. inæquales.

3. Fiquinoctiales flores aperiuntur certâ, et positivâ diei horâ, et plerumque etiam determinatâ horâ quotidie recluduntur.Observant hi horas Europæas,s.æquales. Vigilie Florum solarium vulgatissimæ sunt.
$F l$. suec. et $H$. ups.
oritur, occidit.

I. Leontodon

627. Taraxacum

2. - -

628. Taraxaconoides 4

5. $6 .-8.9$.

3. - -

629. Chondrilloides 7.

4. Hypochœris 631. pratensis

5. - -

1. hispida

6. - -

2. Chondrilloides

7. Hieracium

635. Pilosella

637. Pulmonaria

9. - -

639. fruticosum

I. latifolium

10. - -

3. rubrum

12. Crepis

640. tectorum

13

I. alpina

6. rubra

I. magnx

I 5. Picris

16. Sonchus

17. - -

642. repens

I8. - -

643. levis

I9. - -

644. Iapponicus

20. Lactuca

I. belgicus

I. sativa

2.1. Scorzonera

3. tingituna

22. Tragopogon 648. luteum

23. - -

24. - -

25. Lapsana

26. - -

27. - -

3. Columna

28. Cichoreum 650. scanense

29. Nymphæa 427. alba

30. Calendula 712. arvensis

I. - -

32. Papaver

2. africana

4. nudicaule

4. Dalech.rmpii

I. Rhagadiolus

6.

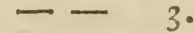

7. $8 .-2$.

9. - I2. I.

8. - - 2 .

6. $7 .-2$.

6. - - 5.

7. - - I. 2.

6. $7 .-3 \cdot 4$.

4. 5. - I0. 12.

5. 6. - I I.

6. 7. - - I. 2.

4. 5. - I2. 9 .

6. 7. - 10. 12 .

5. - II. I2.

7. - I 2 .

6. $7 .-2$.

$7 . \quad$ - 10.

4. 6. - 10 .

3. 5. - 9. 10 .

5. 6. - II.

6. 7. - 12.4.

4. Rhagadioloides 7. 8. - - 2.

3. glutinos.t 5. 6. - I0. 
33 Hemerocallis

34. Convolvulus

35. Malva

36. Alyssum

37. Anthericum 267. album

38. Arenaria 376. purpurea

39. Anagallis

40.

4I. Portulaca

42. Dianthus 7 . prolifer

44. - -

$45 .=$

$46 .--$
.7. fulva

2. rectus

4. belvula

2. crystallinum

Io. ne politanum

5. linguiforme
5.

5.

6.

-7.8 .

9. $10 .-1$.

Alyssoides T. 6 .

7.

9. $10 .-2.3$.

8.

7. 8.

9. I O. - II.I2.

8. - - I.

7. $8 .-2$.

9. $10 .-3.4$.

I0. II. - 3 .

7.

8. $-\frac{}{2}-3$
(.

Horologium Floræ ex sequenti tabulâ formandum, postquam Meteorici, et Tropici flores exclusi sunt, de quibus alibi.

\section{(2) (c)}

3.- Tragopogon luteum 22.

4.-Leontodon Taraxonoid. 2.

4.5-Picris magna Is.

Cichoreum scanense 28.

Crepis tectorum i2.

-.6. Scorzonera tingitaina $2 \mathrm{I}$.

5. - Sonchus lavis 17.

Leontodon Tarasacum I.

Crepis alpina 3 .

Tragopogon Columna 23. Lapsana Rhagadiolus 25.

glutinosa 27.

Convolvulus rectus 34 .

6. - Hypoclworis pratensis 4.

- Hieracium fruticosum 9.

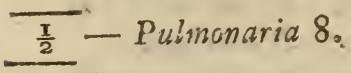

Crepis rubra $\mathrm{I} 4$.

Sonchus repens I 6.

-7. belgicus 19.

7:-Leontodon Chondrilloid.3. Hieracium latifolium $\mathrm{I} 0$. Sonchus lapponicus I8.
(2) 8

- Lactuca sativa 20. Calendula africana 3 r. - Nymphrea alba 29.

- Arithericum album 37.

- 8. Hypochœris bispida 5.

- Lapsana Rhagadiol. 26. Mesembr. barbat. 43 .

9. - Hieracium Pilosella 7.

- Anagallis rubra 39.

- Dianthus prolifer 42. 8.9. Leontodon Taraxacum I.

9. -Hypochœris Chondrill.6.

I0. -Malva belvula 35.

- Arenaria purpurea 38.

-Mesemb.crystallinum 44.

- o.Lapsana glutinosa 27.

-Lactuca sativa 20.

-Scorzonera tingitana $2 \mathrm{I}$.

I O. I I.Mesembr. neapolit. 45.

- I I.Crepis alpina I 3.

- Tragopogon Columne23.

- 12. Sonchus levis 17.

- I2. lapponicus 18. $\mathrm{Hy}-$ 


\section{ADUMBRATIONES.}

Is (c) Hypochœris Chondrill. 6. $\left.\right|_{-3} ^{\infty}$. Mesembr. nexpolit. 45. Malva helvula 35 .

Dianthus prolifer 42.

-2. Hieracium latifolium 10. Crepis rubra I 4.

2. Hypochoris bispida 5. Hieracium Pulmonaria 8. Sonchus belgicus 19. Lapsana Rhagadioloid.20. Mesembr. barbatum 43. -3. Arenaria purpurea 38.

3. Leontodon Chondrilloid.3. Calendula arvensis 30.

Calendula africana Hort. ups. 274. n. 2. vigilias subit inter horam sextam, et septimam matutinam, vigilans in horam pomeridianam quartam, si tempestas diei fuerit sicca; at verò si vigilias non adsumat, seu non aperiat flores horâ septimâ matutinâ, pluvice hac die cadent, constanti lege; imbres autem ex tonitru evitare non facilè didicit.

Soncbus sibiricus si noctu claudatur, proxima dies plerumque serena erit; si verò aperto flore per noctem vigilet, insequens dies plerumque erit pluviosa.

Dormientes per noctem plantas, foliis conniventes, tetigimus \$. I 33 .

FRUCTESCENTIA comprehendit tempus, quo semina matura dispergunt Plantæ.

HORDEUM flosculis omnibus hermaphroditis, seminibus corticatis Hort. ups. 22.

1732. Lapponia. I750. Upsaliæ. Maturescit. Satum Maji 3r. Satum Mrartii 6. Uplandia I10. Dissec. Fulii 28. Dissec. Augrusti 4. Scania 90. Maturuit dieb.58. Maturuit dieb. 155. Lapponia 60. Maturescit Hordeum citiùs in australi Scanià, et Boreali Lapponiâ, quàm in intermediâ Uplandiâ.

DEFOLIATIO est tempus autumnale, quo arbores folia dejiciunt, eoque indicant progressum autumni, et insequentis hyemis.

Fraxinus inter primas defoliatur, et inter ultimas frondescit.

Colchici primi flores observandi. 
VIGENDI tempus comprehendit annos, quibus plantæ vivunt; facilè antui numerantur vel ex circulis concentricis, s. ex anuulis resinosis in Caudice detruncato. Annulis resinosis, s. internis plericue Truncinumerantur. Quercus Melandixe nata 1581 , ætate 260. it. clank. 68. Pinus Wermelandica nata 1337 . xtate 409. it. w. goth. 247.

Ramis annotinis numerantur ætates Pini, Cedri, Mali, Pyri \&c.

Cbronica byemum intensiorum, aut remissiorum ex annulis internis datur in plerisque arboribus, præsertim in Quercu.

Botanici hucusque in plantarum numerosissimarum dignotione ozcupati, et objectorum varietate inundati, nequivere more astronomorum observationes instituere, licèt inferiora sequendo, longè tamen majorem publico usum suis observationibus subministraturi.

Calen.taria Flore quotannis conficienda sunt in quavis Provinciâ, secundùm Frondescentiam, Efflorescentiam, Fructescentiam, Defoliationem, observato simul Climate, ut inde constet diversitas Regionum inter se. Horologia Flore sub qunvis Climate elaboranda suni secundùm Vigilias planiarum, ut quivis sine horologio, aut sole horam diei enumeratam habeat.

Mlappe vegetantes, qux indicant ubique Regionem, Clima, et Terram.

Usus ex prædictis resultaret de Naturâ Telluris summus.

Clima ex progressu anni secundùm Frondescentiam, et Defoliationem, unde Calor, Frigusque loci summum, notissimum Bctanico erit.

Thermometrum Botanicum erit nostrum, cujus punctum congelationis $\mathrm{O}, \mathrm{Calor}$ verò aqua coquentis 100 .

Autumnales plantæ sunt Virginicæ, quæ florent latissimè Septembri, et Octobri, cùm æstas sit ipsis in patriâ gratissima, adeoque in Sueciâ difficillimè semina maturant.

Brumales sunt Capenses, quarum flores calore grato sub ipsâ Brumâ provocantur, dum astas in patriâ regnat, aliàs frustra proliciendi.

Vernales sunt Alpince omnes, cùm in Alpibus hyems excipiat ver vix gustatâ æstate, adeoque citissimè florescant, et fructescant. 
Biferce, qux bis in anno florent, vere scilicet, et autumno, more patrio, uti Indice facilè omnes intra Tropicos.

Frigide gradum caloris trigesimum vix ferunt, uti
Alpine
Sibirice
Canadenses
Germanica
Belgicre .
Anglice
Gallice supra Parisios.

Temperate hyemes viginti octo graduum frigoris non ferunt:

Narbonenses

Lusitanice

Italice

Hispanica.

Syriace.

Calidæ ferunt Caloris gradum quadragesimum, sed frigoris gradu duodecimo suffocantur.
Indice orient.
Americes merid.
Egyptiace
Canarienses

Frigide in Calido primùm luxuriant, mox flaccescunt, pereunt.

Calide in Frigido crescere cessant, defoliantur, sterilescunt. 


\section{I I. V I R E S.}

336. Vires plantarum a Fructificatione (8.5) desumat Botanicus (7), observato Sapore $\left(3^{6} 5\right)$, Odore (362) Colore $(364)$, et Loco (357.)

Auctores sententiæ fuere Hermannus, R. J. Camerarius, Hoffmannus, et alii.

Dissertatio de Viribus plantarum, a F. Hasselquist U'psalixe

1747. propugnata, hoc de Viribus Caput explicat.

Falsæ sunt veterum Theorix de viribus plantarum ex $A$ strologiâ \$. 47. Signaturậ \$. 47. Chemiâ \$. 48.

Probant Urdines naturales veritatem aphorismi $\$ .77$.

337. Plantæ, quæ Genere conveniunt $\left(\mathrm{r}_{5}\right)$, etiam virtute conveniunt; qux Ordine Naturali (77) continentur, etiam virtute propiùs accedunt; quæque Classe Naturali congruunt, etiam viribus quodammodo congruunt.

Genere convenientes easdem possident vires:

Convolvuli : Scammonium, Mechoacanna, Turpethum, Soldanella.

Allia : Moly, Porrum, Cepre, Victorialis.

Lauri: Cinnamomum, Malabatbrum, Cassia, Camphora, Sassafras, Benzoë.

Euphorbiæ: Esula, Cataputia, Tithymalus. Artemisiæ: Abrotanum, Absintbium, Cinna, Seripbium.

ORDINE maturali, et viribus conveniunt:

Columniferæ: Malva, Althrea, Alcca, Gossypium. Scitamina: Zingiber, Cardamomum, Galanga, Zedoaria, Costus, Grana paradisi, Curcuma.

Orchideæ: Orchis, Satyrium, Serapias, Epidendrum. Multisiliquæ: Pronia, Aquilegia, Aconitum, Delphinium, Nigella, Helleborus, Ranunculus, Pulsatilla. Contortæ: Apocynum, Cynanchum, Asclepias, \&c. 


\section{GRES.}

CLASSE, et viribus congruunt:
Gramina,
Tricocce,
Compositie,
Sarinentacere,
Umbellate,
Oleracere,
Filices,
Papilionacee,
Lomentacea,
Siliquose,
Verticillate.

338. Graminum (77: r4) Folia Pecoribus, et Jumentis lreta pascua; Semina minora avibus, majora hominibus esculenta sunt.

Folia graminum primarium pabulum animalibus phytivoris.

Semina minora Phalaridis, Panici, Milii, sunt gratissima passeribus, et gallinis.

Cerealia sunt Semina majora graminum, qux hominibus quotidie in cibum veniunt: Oryza, Triticum, Secale, Horilzum, Avena, Milium, Panicum, Holcus, Z:zania, Mays: excepto fortè solo Lolio, nisi arte præparato.

339. Stellatæ $(77: 44)$ diureticæ sunt.

Exempla: Rubia, Asperula, Aparine, Galium.

340. Asperifolix $(77: 43)$, magis, minùsve oleracex, mucilaginosæ, et glutinosæ sunt.

Oleracex: Anchusa, Borrago \&c.

Mucilaginosæ: præcipuè Consolidæe majoris Radix, quæ inter glutinosas primaria est.

341. Luridæ (77: 33) sunt plantæ suspectæ.

Fœtidæ: Solanum, Hyoscyamus, Nicotiana, Atropa, Mandragora, Datura.

Dementantes, et Narcoticæ: Atropa, Mandragora, Nicotiana, Hyoscyamus, Melongena, Lycopersicon apud nostrates. Corrosivuin maximè : Capsicum.

342. Umeellat $(77: 22)$ in siccis aromatica, calefacientes, et pellentes; in aquosis autem Venenatæ sunt; radice, et seminibus pollent. 
Aquoso loco natx, et venenatre sunt Cicuta, Oenanthe, Sison,

Phellanilrium, Apium palustre.

Pellentes sudorem, urinam, flatus, menses, lac, sunt Assa fotida, Levisticum, Angelica, Imperatoria, Pimpinella, Peucedanum, Opoponax, Galbanum, Carvi , Cyminum, Daucus, Meum, Føniculum.

343. HEXANDRIE (68) radices secundùm saporem, et odorem edules, aut noxia sunt.

EDULES sunt Radices inodoræ: Martagon, Tulipre, Ornithogala.

VENENATE secundùm odorem virosum sunt Gloriosa, Scilla, Hyacintbus, Antbericum, Leucojum, Narcissus, Corona imperialis.

344. BICORNEs ( $77: 24$ ) adstringunt, sed Baccæ acidæ esculentæ sunt.

Adstringunt: Erica, Pyrola, Vaccinium, sed maximè Uva ursi. Baccæ acidæ, et esculentæ in hac gente sunt $V$ accinia, Myrtilli, Oxycocci, Uve ursi, Arbuti, Gujacana, Melastome.

345. Icosandriza ( 68 ) fructus pulposus est esculentus.

Pulposi fructus in hac classe sunt:

Pomacei 77: 37: Mala, Pyra, Granata, Crategi, Mespila, Sorba, Ribes.

Senticosi $77: 35$ : Cynosbata, Ruba, Fraga.

Drupacei 77 : 38: Amygdala, Persica, Pruna, Armeniaca, Cerasia.

Arbustivi 77:39: Guajava, Eugenia.

\section{Polyandria (68) plerumque venenata est.}

Multisiliquæ 77: 23: Aconitum, Antbord, Aquilegia, Staphisagria, Delpbinium, Helleborus, Apium risus, Clematis, Pulsatilla, Pronia.

Rhœades 77: 30: Papaver, Chelidonium, Actrea. Alix: Euphorbia, Cambogia, Peganum. 
347. VeRTICILlate $\left(77: 5^{8}\right)$ sunt fragrantes, nervinæ, resolventes, et pellentes; Folia virtute pollent.

Fragrantissimæe: Marum, Dictamnus, Satureja, Tbjmus, Origanum, Majorana, Ocymum, Pulegium, Mentha, MIclissa, Lavandula, Rosmarinus, Salvia, Horminum, Sclarea.

Fragrantes actione in nervos resolvunt. Pellunt flatus, menses, Lac, Genituram.

348. Siliquos $=$ ( $77: 57)$ aquosæ, acres, incidentes, abstergentes, et diureticæ sunt, exsiccatione imminuitur virtus.

Exsiccatione cùm virtus imminuatur, vivæ itaque a Medicis sunt adhibendæ.

Incidentia attenuant viscida acredine, hinc tumores œdematosos, et frigidos resolvunt: Cocblaria, Armoracia, Sisymbrium \&c.

349. Columnifere $(77: 34)$ muciliginosæ, lubricantes, obtundentes, et maturantes sunt.

Lubricant inviscando acria in Tussi, Stranguriâ, Nephritide, Colicâ, Excoriatione, adeaque Dolores leniunt. Maturant emolliendo.

350. Papilionaceorum $(77: 55)$ Folia Jumentis, et Pecoribus; Semina, variis animalibus esculenta, sunt farinacea, et flatulenta.

Flatulenta, et esculenta sunt Fabe, Vicise, Pisa, Phaseoli, Ciceres, Lentes.

Quadrupedibus præstantissimum pabulum suppeditant Trifolium, Medicago, Trigonella, Hedysarum, Vicia, Loius, Lathyrus.

351. Syngfinesia (68). Compositorum $(77: 21)$, in Medicinâ receptissima, communiter amara est. 
Amara: Eupatorium, Tanacetum, Balsamita, Santolina, Absintbium, Abrotanum, Artemisia, Ageratum, Matricaria, Chamomilla, Cotula, Acmella, Auricula muris, Taraxacum, Cichoreum, Carduus maria, Carlina, Carduus benedictus.

352. OrCHIDEIE $(77: 4)$ sunt aphrodisiacæ.

Aphrodisiaca inter primaria sunt Vanilla americanorum, Salep orientalium, Satyrium Europæorum.

353. CONIFER $2\left(77: I_{5}\right)$ sunt resiniferæ, et diureticæ.

Resiniferæ sunt omnes, adeoque et sempervirentes.

Diureticæ, urinam violaceam reddentes, sunt Terebinthina, Juniperus, Sabina, Clibanum, Pinus, Abies, C'upressus.

354. Cryptogamia ( 68 ) Vegetabilia sæpius suspecta continet.

Filices (77:64.) odore gravi ingratæ sunt.

MIusci (77:65.) itidem ingratè olent.

Alge $(77: 66$.) paucissimæ esculentæ sunt, et plures purgant.

Fungi (77:67.) anceps cibus Plinio.

355. Plantæ floribus nectario a petalis distincto ( I 10 ) communiter venenatæ sunt.

Nectaria distincta observantur in Aconito, Helleboro, Aquilegiâ, Nigellâ, Parnassiâ, Epimedio, Clutiâ, Kiggellariâ, Hyacintho, Stapeliâ, Asclepiade, Mirabili, Nerio, Narcisso, Zygophyllo, Dictamno, Meliantho; quæ omnes venenatæ.

356. LaCtescentes plantæ communiter venenatx sunt, minùs autem semiflosculosæ $(77: 21$. a.)

Lactescentes venenatæ sunt pleræque. 
Contortæ 77:29. Rhœades77:30. Tricoccæ 77:47. Variæ.

$\begin{array}{llll}\text { Rauzvolfia } & \text { Papaver } & \text { Euphorbia } & \text { Rbus } \\ \text { Thevetia } & \text { Argemone } & \text { Cambogia } & \text { Ficus } \\ \text { Cerbera } & \text { Chelidoniun } & \text { Dalechampia } & \text { Acer } \\ \text { Plumieria } & \text { Bocconia } & \text { Jatropha } & \text { Melia } \\ \text { Tabernemont. } & \text { Sanguinaria } & & \text { Agarici. } \\ \text { Periploca } & & \\ \text { Apocynum } & & \\ \text { Cynanchum } & & & \\ \text { Ceropegia } & & & \\ \text { Asclepias. } & & & \end{array}$

Semifiosculose ( $77: 2$ I. a.) lactescentes vix venenatæ sunt: Prenanthes, Chondrilla, Hieracium, Crepis, Hypocbocris, Picris, Hyoseris, Leontodon, Tragopogon, Laciuca, sed sunt etiam Lactucæ species aculeatæ, virosæ, maximè venenatæ.

Campanulacere $(77: 32)$ sunt partim venenatæ, uti Lobelia: partim vix noxiæ, uti Campanula.

357. Locus siccus sapidiores, succulentus insipidas magis, aquosus sæpius corrosivas reddit.

Aquaticæ plurimæ plantæ acres, et corrosiræ sunt: $R a-$ nunculus, Calla, Nympheea, Sium, Phellandrium, Cicuta, Persicaria, Armoracia, Sisymbrium.

Vernales hanc ob causam etiam plurimæ acres.

Succulento solo natæ plantæ magis inșipidæ: ut Oler pleraque.

Sicco solo proveniunt præstantissima aromata: Cinnamomum, Rosmarinus, Thymus, Salvia, Origanum, Clinopodium, Hyssopus, Lavandula, Melissa, Caryophyllus. Aromata sapidissima sunt sicca, et plantæe exsiccatæe evadunt sapidiorcs.

Fructus horæi in humidis, et umbrosis austeriores; in siccis, et soli expositis dulciores evadunt.

353. Qualitates plantarum, in quibus vires subsistunt, indicat Seper, Odor, Color.

Sensus exterrni sunt instrumenta naturalia, quibus explorantur plantarum qualitates.

Insipida, et inodora vim medicam vix exercent.

Sapidissime, et odoratissima verò semper maximam rim possident. 
Destructo sapore, et odore in plantis etiam vis castratur, ut in Foeculis, et Magisteriis Calbe, Ari, Jatrophe, Elaterii.

Masticatione vires a sapido provenientes optimè explorantur.

359. Sapide, et Suaveolentes bonæ sunt; Nauseose, et Graveolentes venenatæ sunt.

Notiones hre sensibus omnium animalium inscriptæ sunt secundùm texturam cujuscumque corporis.

Graveolentes malæ sunt: Fungi, Cotula, Sambucus, Actra, Aconitum, Helleborus, Veratrum, Asarum, Anagyris, Solanum, Datura, Nicotiana, Hyoscyamus, Tagetes, Cassia, Stachys, Doronicum, Colocynthis, Coriandrum, Busus, Cynoglossum, fuglans, Opium.

360. Contraries qualitates contrarium effectum exercent.

Contrarii morbi contrariam causam agnoscunt, quæ exercent contrarium effectum.

Morbi ergo Morbis curantur.

361. Agunt omnes plantæ vel Olido in nervos, vel Sapido in fibras, vel utroque in fluida.

Sapida nunquam agunt in nervos, nec Olida in fibras musculares.

Fluida immutantur a sapidis; evacuantur et a sapidis, et $a b$ olidis.

362. Ambrosiaca sunt analeptica, Fragrantia orgastica, Aromatica excitantia, Tetra stupefacientia, Nauseosa corrosiva.

Ambrosinca agunt, uti Ambra, Moschus, Zibethum. Asperula, Abelmosh, Geranium moschatum, Malva moschata, Milium, Aira.

Fragrantia gratissimo odore sese commendant.

Flores Croci, Cheirantbi, Polianthis, Jasmini, Lilii, Tilia, Viole. 
Herbæ: Lavandula, Tbymus, Majorana, Ocymum, Origanum, Satureja, Mrelissa, Marum.

Aronatice Odore, et Gustu ferè conveniunt.

Cinnamsmum, Laurus, Sassafras, Camphora, Macis,

Cardamomum, Caryopbyllus, ILyristica, Acorus, Ammi, Angelica, Citrus.

Graveolentia singularia sunt:

Alliacea: Allium, Cepa, Porrum, Alliaria, Scordium, Peliveria, Assa feetida.

Hircina: Orcbis, Vulvaria, Hieracium fotidum, Geranium rolertianum.

Tetra odore ingrato notissima:

Stacbys fatida, Cotula fetida, Tagetes, Opium, Cannabis, Ebulus, Anagyris.

Nauseosa, quæ ingesta a natura respuuntur.

Veratrum, Helleborus, Convallaria, Asarum, Nicotiana, Colocynthis.

$3^{6} 3$. SAPIDA in Fluida, et Solida agunt.

Dulcia Edulcorantia, et Impinguantia,

Incidentia, et Corrodentia Acria,

Pinguia Obtundentia, et Emollientia,

Inspissantia, et Adstrigentia Stiptica,

Acida Refrigerantia, et Attenuantia,

Balsamica, et Tonica Amara,

$V$ iscosa Mucilaginosa, et Lubricantia,

Penetrantia, et Abstergentia Salsa,

Aquosa Mundificantia, et Humectantia,

Absorbentia, et Exsiccantia Sicca sunt.

Qualitates contrariz.

Aquosa, et Sicca

Pinguia, et Stiptica

Aeida, et Amara

Dulcia, et Acria

Salsa, et Viscosa
Qualitates conformes.

Aquosa, et Viscosa

Dulcia, et Pinguia

Acida, et Salsa

Acria, et Amara

Sicca, et Stiptica. 
Vires Contrarie in fluidis.

Munlificantia, et Absorbentia

Refrigerantia, et Balsamica

Edulcorantia, et Incidentia

Obtundentia, et Inspissantia

Mucilaginosa, et Penetrantia

VIRES CONTRARIE in solidis.

Humectantia, et Exsiccantia

Attenuantia, et Tonica

Impinguantia, et Abstergentia

Emollientia, et Adstringentia

Lubricantia, et Corrodentia.

$\begin{aligned} \text { Exempla Aquosi: Aqua, } & \text { Sicci: Farina, } \\ \text { Viscosi: Gummi, } & \text { Acris: Sinapis, }\end{aligned}$

Pinguis: Oleum, Salsi: Sal,

Dulcis: Saccharum, Amari: Bilis,

Acidi: Acetum, Stiptici: Galla.

364. Color Pallidus insipidum, Viridis crudum, Luteus amarum, Ruber acidum, Albus dulce, Niger ingratum indicat.

LUTEA amara:

Gentiana, Aloë, Chelidonium, Curcuma, Flores lutei, RUder acidum:

Bacce Oxycocci, Berberidis, Ribis, Rubi, Mori.

Poma acida, Hippophaës, Sorbi, Rose, Cerasi.

Herbæ versùs autumnum rubescentes:

Acetosa, Oxalis, Lapatbum sanguineum, Brassica.

Viridis crudum: Folia, et Fructus immaturi.

Pallidus insipidum:

Albus dulce:

Lactuca, Cichoreum, Asparagus.

Ribes, Rubus, Mala dulcia, Pruna alba.

Nigra ingrata, et sæpius venenata:

Eacce Atrope, Actree, Coriarice, Solani, Tini, Empetri, Padi.

Acidi exploratores sunt colores cærulei, s. violacei. e. gr.

Crotonis, Violæ, aliorum vegetabilium.

Cærulei colores ab acido rufescunt, ab alcali affuso virescunt. 
Papyro cæruleâ usus est Tournefôrtius, quæ ab affuso succo plantæ mutata indicabat ejusdem qualitatem acidam, aut alcalinam.

365. Oeconomicus Usus plantarum generi humano utilissimus est.

Usuales plantas pro Pane, Cibo, Potu, IEdificiis, Utensilibus, Instrumentis, Opificiis, Tincturis probè observet ubique locorum Botanicus, et describat.

Flora Oeconomica ejusmodi nonnulla collegit.

Itinera nostra Oelandicum, Gothlandicum, Westrogothicum, Scanicum, varia suppeditarunt.

Usus plantarum pro pabulo animalium sollicitè inquirendus. $P$ an suecicus hujus fundamenta jecit.

Usus plantarum pro Oeconomiâ universali nature sedulò indagandus.

Oeconomia Naturce (Dissert. ac.) ejusmodi plura conquisivit.

In Scientiâ Naturali Principia veritatis

Observationibus confirmari debent. 


\section{T A B ULA I. \\ F O L I A I M P I C IA.}

Fig. I. Orbiculatum.

2. Subrotundum.

3. Ovatum.

4. Ovale, s. Ellipticum.

5. Oblongum.

6. Lanceolatum.

7. Lineare.

8. Subulatum.

9. Reniforme.

10. Cordatum.

I I. Lunulatum.

I2. Triangulare.

I3. Sagittatum.

I4. Cordato-Sagittatum.

I5. Hastatum.

I6. Fissum.

I7. Trilobum.

I8. Prxmorsum.

19. Lobatum.

2o. Quinquangulare.

21. Erosum.

22. Palmatum.

23. Pinnatifidum.

24. Laciniatum.

25. Sinuatum.

26. Dentato-sinuatum.

27. Retrorsum sinuatum.

28. Partitum.

29. Repandum.

30. Dentatum.

3 I. Serratum.
32. Duplicato-serratum.

33. Duplicato-crenatum.

34. Cartilagineum.

35. Acutć crenatum.

36. Obtusé crenatum.

37. Plicatum.

38. Crenatum.

39. Crispum.

40. Obtusum.

4I. Acutum.

42. Acuminatum.

43. Obtusum acumine.

44. Emarginatum acutè.

45. Cuneiforme emarginatum.

46. Retusum.

47. Pilosum.

48. Tomentosum.

49. Hispidum.

50. Ciliatum.

5 I. Rugosum.

52. Venosum.

53. Nervosum.

54. Papillosum.

55 . Linguiforme.

56. Acinaciforme.

57. Dolabriforme.

58. Deltoides.

59. Triquetrum.

6o. Canaliculaium.

6. Sulcatum.

62. Teres. 


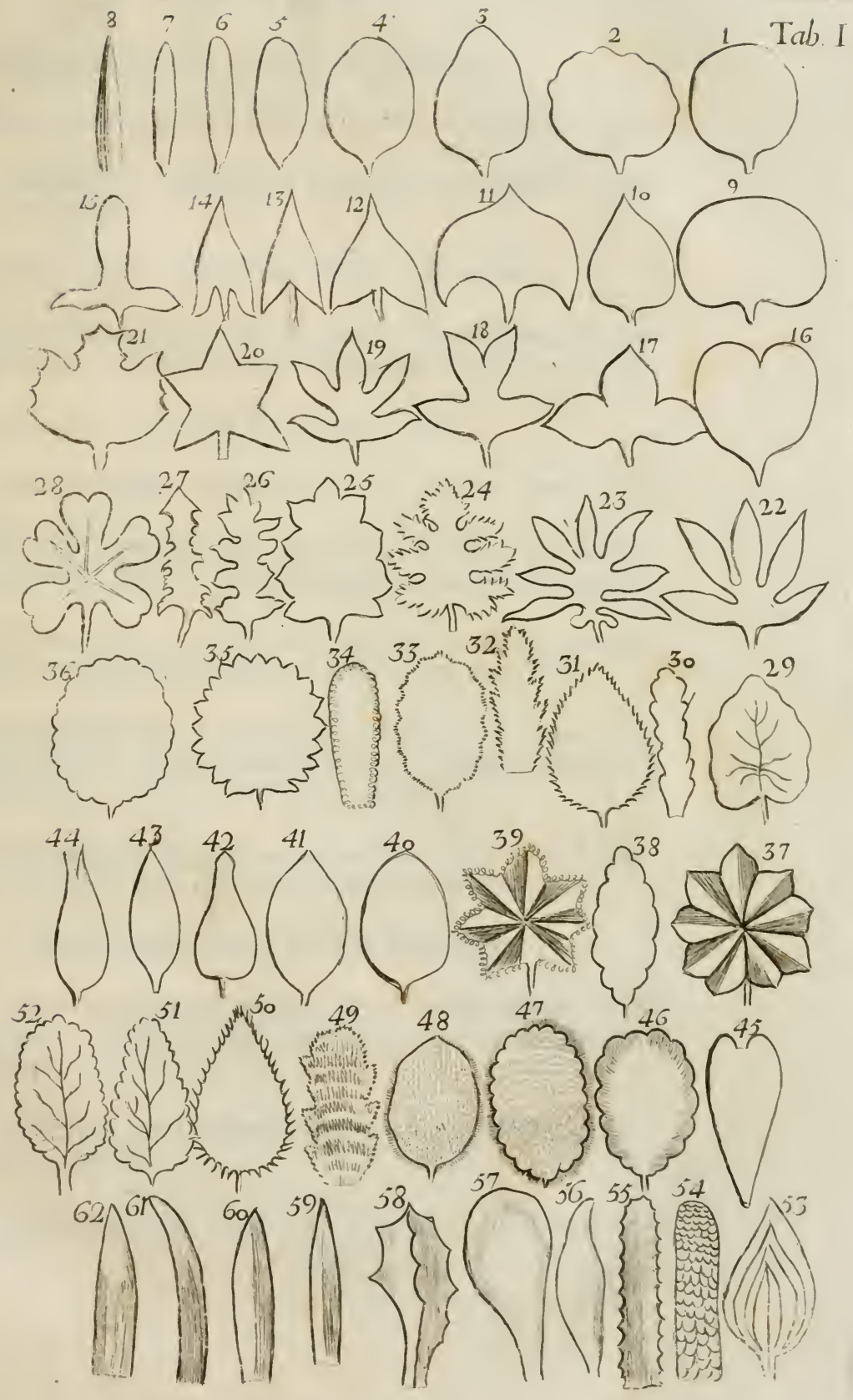




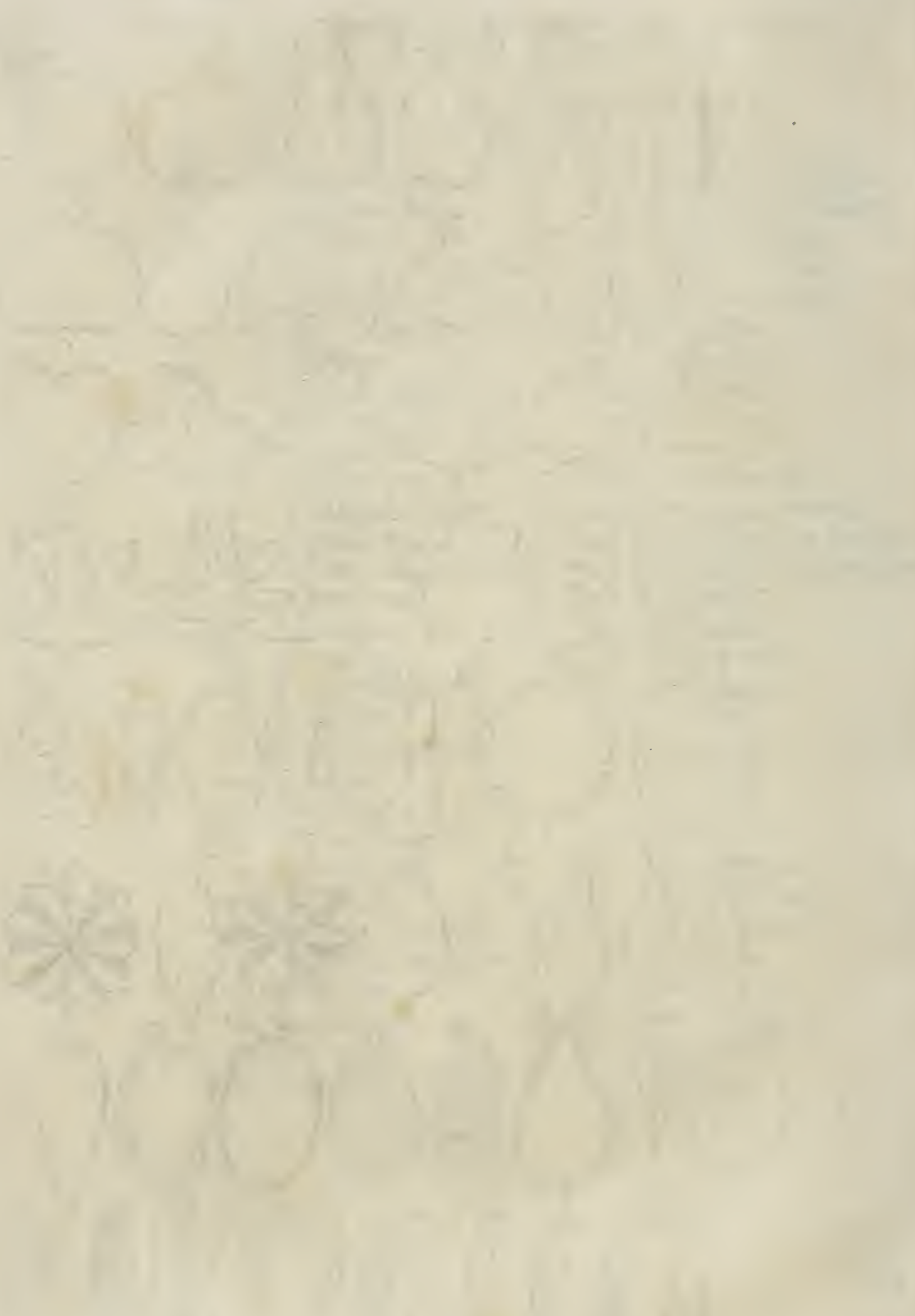




\section{TYRO.}

furies Planite omnes sibi reddat notissimas.

Vulgatissimas plantas a facie Herbationibus discat dignoscere.

Colligat, Exsiccet, Adglutinet ipse plantas majores, quatquot poterit.

Fractificationis partes primarias discat distinguere.

Classes, et Or.tines systematis sibi reddat familiares, et simpliciores, eviduntioresque flores ad eosdem reciucat.

Demonstrationibus in Horto frequenter adsit.

Terminos artis secundùm definitiones sibi habeat perspectos.

Genera sibi nota, circiter 50. secundùm Genera plantarum examinet, collatis fructificationibus cum charactere.

Characteres Gencricos 50. proprio marte, eadem methodo conficiat, et sacundùm Genera plantarum emendet.

Descriptiones specierum, 60 circiter, conficiat ex lege artis, incipiendo a simplicissimis plantis, procedendo ad dificiliores, quas corrigat Professor.

Ignotas sibi plantarum species investiget ipse secundùm Classes, Characteres, Differentiasque systematis.

Principia, et fundamenta Botanices rite intelligat.

Historiam literariam Botanices sibi familiarem reddat ; et in primis Auctores de Speciebus plantarum consulendos.

Synonym. Auctorum, retrogrediendo ad inventores, ercl. vere adsuescat.

Usum plantarum speciebus adscribat, Medicum, et Oeconomicum. 
290

\section{T A B U L A I I. FOLIA COMPOSITA.}

63. Binatum.

64. Ternatum foliolis sessilibus.

65. - ... - petiolatis.

ó6. Digitatum.

67. Pedatum.

68. Pinnatum cum impari.

69. - - - abruptum.

70. - - alternatim.

71. - - - interrupté.

72. - - cirrbosum.

73. - - conjugatum.

74. - - - decursivè.

75. - - articulatè.

76. Lyratum.

77. Biternatum, Duplicato-ternatum.

78. Bipinnatum (Saurag.), Duplicato-pinnatum.

79. Triternatum, Triplicato-ternatum.

8o. Tripinnatum ( Sauvag.) sine impari.

8I. - . - cum impari. 


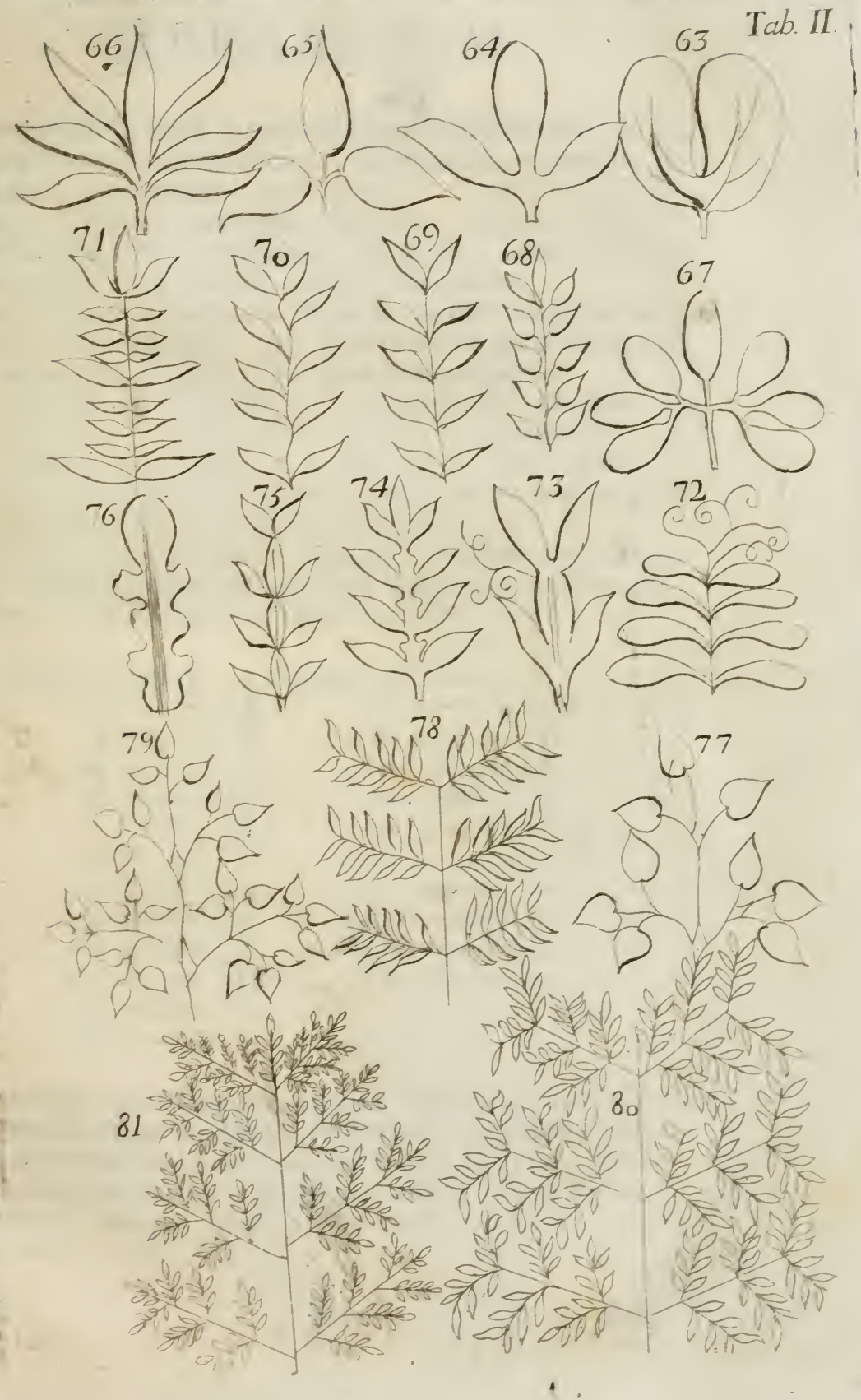





\section{H ER B A R I U M.}

Plantæ methodo datâ (p. 7.) collectæ in Herbarium vivum, aliis Holtum siccum dictum, disponuntur secundùm Systema, ut prompiè evolvantur.

Instructio Herbarii secundùm Systenia sexuale hre est; secundùm aliud quodcunque Systema, observatis observandis, alii eadem ratione disponant.

Arca ex Ligno, claudenda Valvulis duabus longitudinalibus, respondentibus aptè dissepimento perpendiculari. Spatium arcæ internè accuratè determinetur.
Altitulo paris. pedum $7 \frac{\frac{1}{2}}{2}$ a summo ad infimum.

Latitudo - - unciar. I6, preter dissepimentum.

Profunditas - Pedis I, a pectore sc. ad tergum.

Dividatur arca dissepimento perpendiculari in duas partes æquales, a pectore ad tergum.

Locula fiant horizontaliter distincta: Assere crasso lin.6. CLASSIUn loculamenta sint instructa secundùm copiam specierum, uti numeri classium indicant:

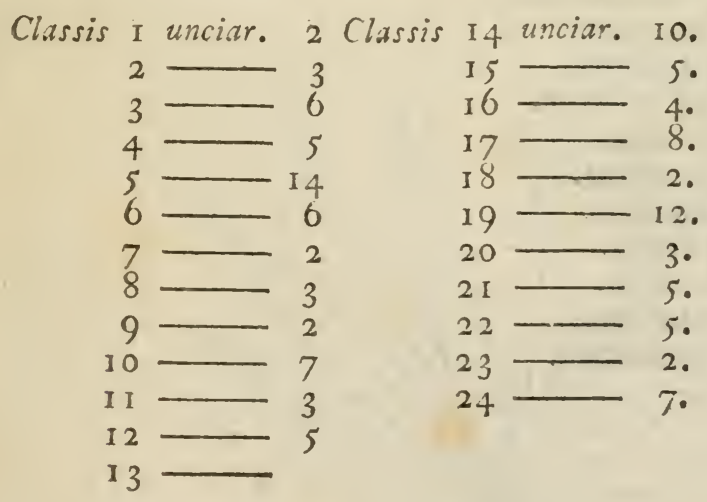

Capit hæc arca 6000 Plantas siccatas adglutinatas. Notatis valvulis arcæ Numero, et Nomine Generum, exactè respondentibus Loculis, intra quxe philyra, genera eadem inclidentes, eorumque numeris ipsa notatx, servantur; absque morâ planta omnis protrahitur, et evolvitur. 


\section{T A B U L A I I I. \\ FOLIA DETERMINATA.}

82. Inflexum.

83. Erectum.

84. Patens.

108. Frons.

85 . Horizontale.

I09. Spathulatum (Sauv.) Folium.

86. Reclinatum.

I I . Parabolicum (Suuv.) Folium.

87. Revolutum.

88. Seminale.

89. Caulinum.

90. Rameum.

91. Florale.

92. Peltatum.

93. Peticlatum.

94. Sessile.

95. Decurrens.

96. Amplexicaule.

97. Perfoliatum.

98. Connatum.

99. Vagituans.

Ioo. Articulatum.

IOI. Stellata.

102. Quaterna.

ro3. Opposita.

104. Alterna.

I05. Acerosa.

zo6. Imbricata.

z07. Fasciculata. 


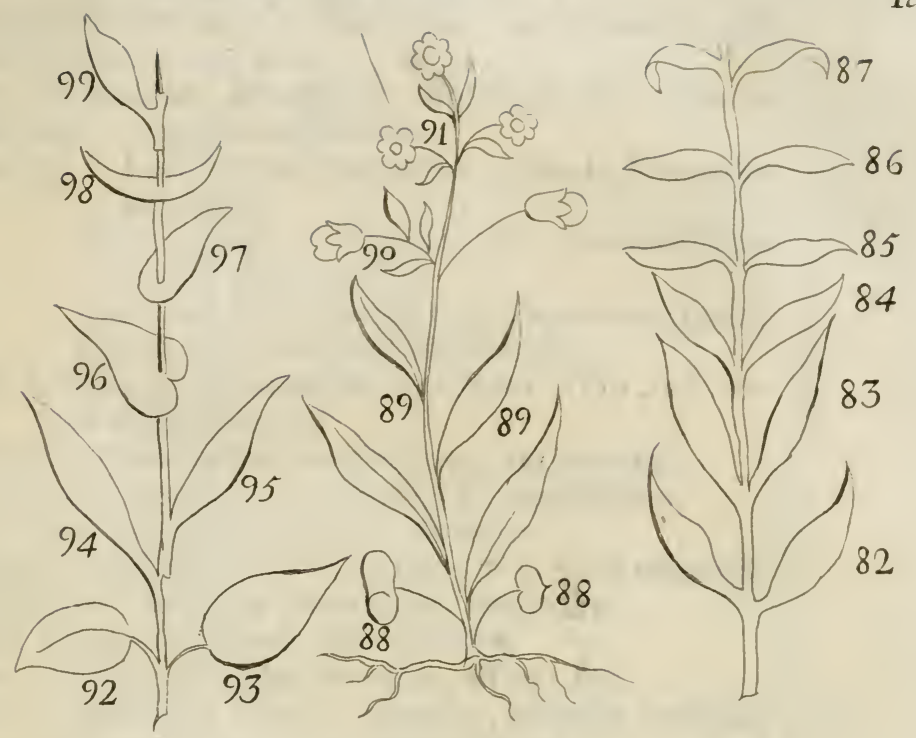

Tab. III

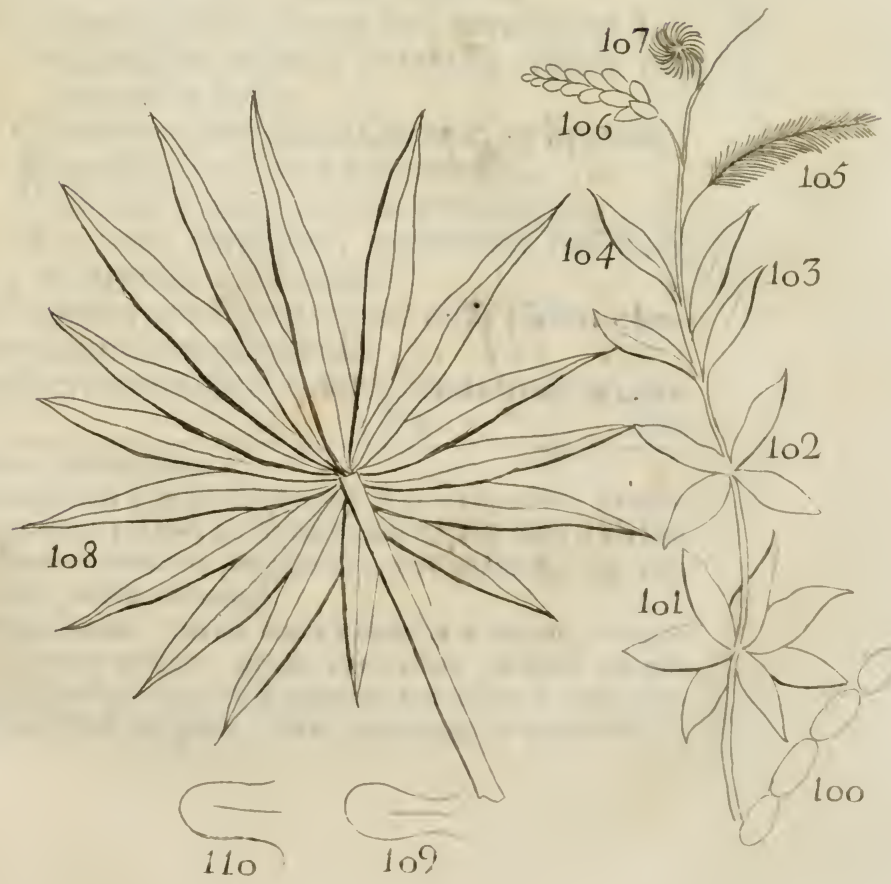




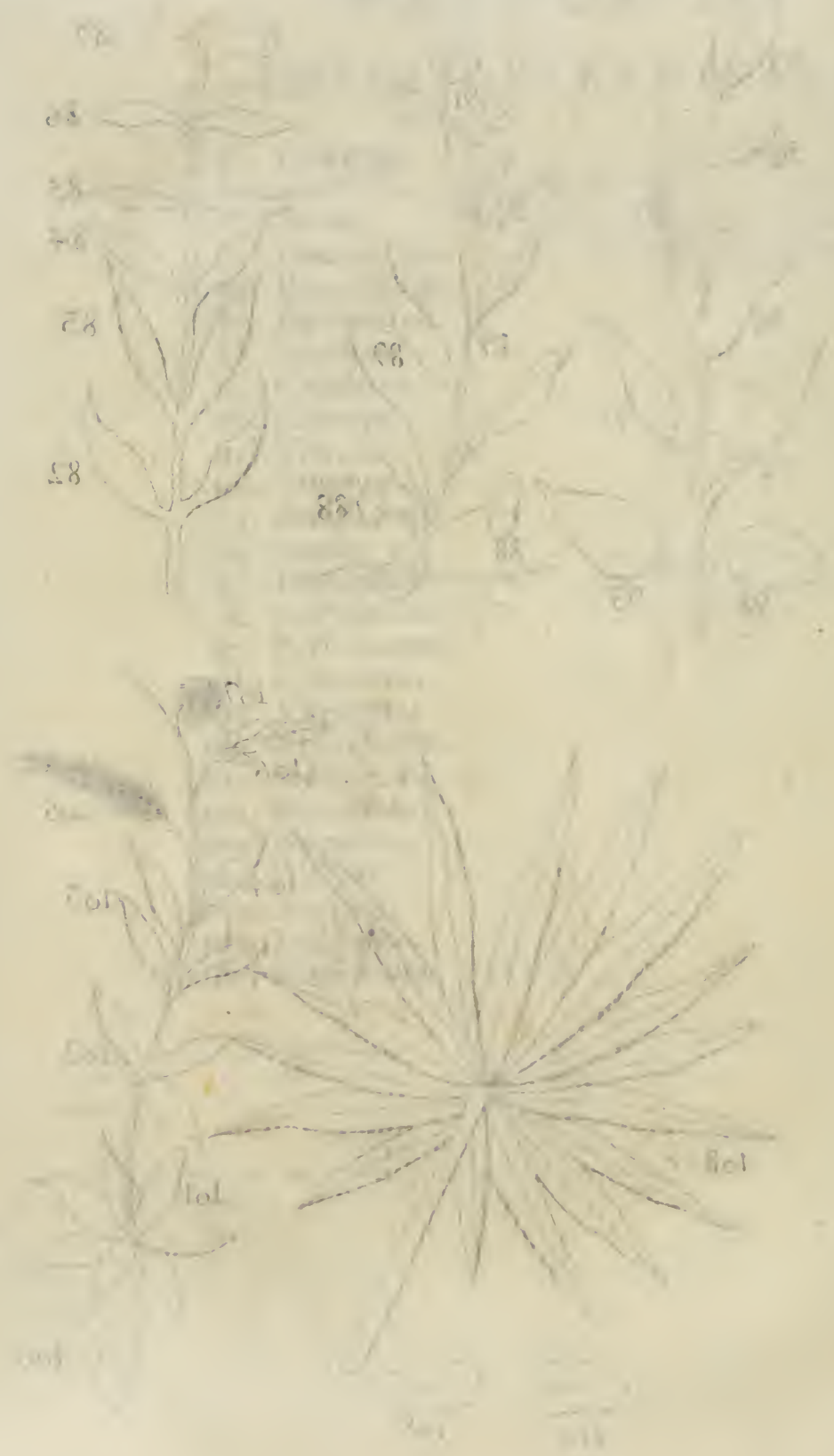




\section{HER B A T O.}

Excursiones Botanica (a) a varils varie liastitu untur; nobis sequentes in usu sunt.

Vestitus levissimus, laxissimus, Botanicis (b) proprius (ubi licet), riegotio aptissimus.

Instrumenta : Libri: Systema naturæ, Flara; Faunaque Regionis.

Microscopium, Acus boianica, Cultellus boianicus, Pluinbago.

Vasculum Dillenianum (c), Papyra colligata.

Capsula cum Aciculis pro Insectis.

Tempus a frondescentia arborum, excepto Sir io, ad earum defoliationem.

in hebdomade omni, xstatis bis, veris semel.

ab horâ 7 matutinâ, in horam 7 vespertinam.

LEGEs serò venientis, Discedentis a Absentis.

Divisionis, Pransionis hora 2, Quietis in h. 4, Secretarii. Vra: Congressus, dispersa ambulatio, Stationes.

Milliaria ad summum duc cum dimidio.

Collectio: Plante præcipuè florentes, MIusci \&c.

Insecta, Amphibia, Pisces, Avicule, plumbo occisæ.

Petre, Minere, Fossilia, in primis Terre.

Demonstratro singula a Professore fiat inira horæ $\frac{\pi}{2}$. indigitentur collecta naturalia omnia cum numeris e libris.

Characteres essentiales Generis, et Speciei.

Singularia in objecto observanda.

Usus Oeconomicus, Medicus primarius.

Usus: Lecta in NATALI oblectant, memoriam facilitant, habitum, et naturam adumbrant.

Tot uno die objecta se offerunt, quot aliàs diebus commilitonum numerum æquantibus. Adver saria, a Secretario collscta, describenda in seros usus.

(a) Herborisationes Gallis dictx. $\mathrm{T}_{3}$

TA-

(b) Vestitus Herborisantis præter Lintea sit Tunica brevis; Ferroralia tenuissima prolixa ab hypochondriis ad talos; Calcei læves; Pileus umbraculo amplissimo, aut umbella, ne via, calor, æestus, sudor delassent.

(c) Vasculum Dillenianum: pelvis semicylindrica e cupro, long:tudine unciarum novem, probe operculata, orificio am plo pro manu, latere plano mojicè concavo pro femo e; ad plantas collectas, aquâ irrigatas, vivas servadias in vesperam. 


\section{T A BU L A IV. \\ C A U L E S.}

Fig. III. Squamosus culmus,

II 2. Repens caulis,

II3. Scapus.

I 4. Articulatus culmus.

I15. Volubilis caulis.

I16. Dichotomus caulis.

II7. Brachiatus caulisa 


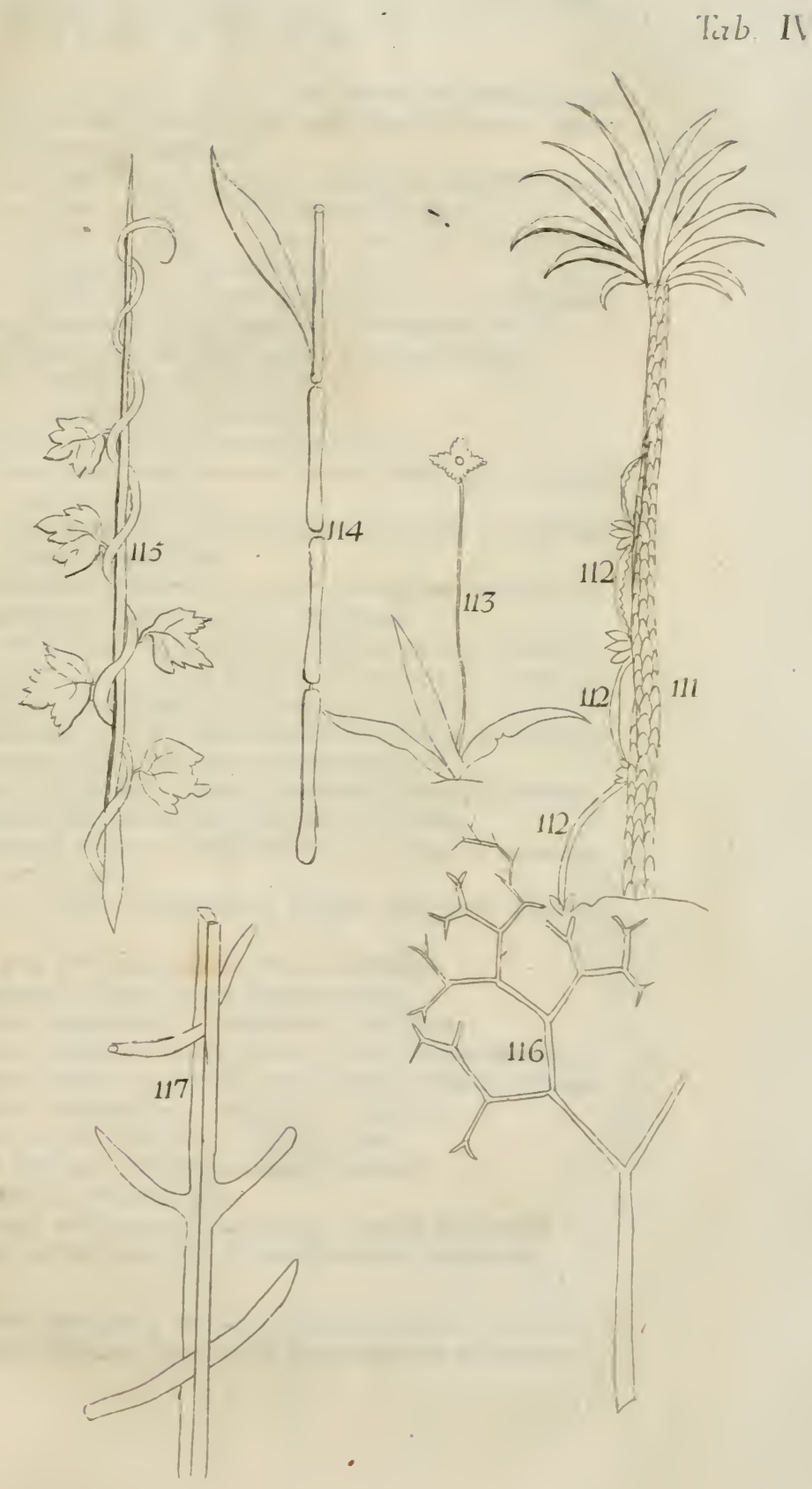





\section{H O R T U S.}

Horfus Botanicus, Paradrsus dictus, alat plantas numerosissimas. HyBRRacula pariete fenestrato, plagæ meridionali obverso, plantas ab hyemis sævitiâ defendant.

Cardarium Caminis tribus, Scamnis, Pulvillo, fenestris juste inclinatis pro plantis Feris. * Thermometr. $12-3^{6}$.

Tepidurium Caminis duobus, Szannis gradatis pro plantis Ethiopicis, Succulentis. Thermometr. 4-12.

Frigidarium Camino unico, spatiosissimumastate pro Demonstrationibus, hyeme pro P1. Marsuetis. Termometr. 2-10.

Museum oblongum, alium, angustum, a tergo horum. Animalia in vitris spiritu vini rep'etis. Lapides in Lythophylacio apto servandi.

EEDS Hortulani, et Professoris prozimè adsint.

IEstivaria hybernaculorum minimorum instar inædificata, plantas ab æstatis injuriâ defendant.

Vaporarium ventre equino Cortice obtecto, in ollis, seminum caldarii pullitiem promoveat.

Solarium, Scamnis gradatis, Ollas plantigeras apricabitur, noctu claudendum fenestris, pro EEthiopicis.

Arex singulæ includantur sepibus vivis, opere topiario formatis.

Perensis spatiosissima, divisa in Pulvillos æquales, resibiles alat syste:naticè Herbas pẹrennes Cicures, vel etiam Vulgares.

Annua spatiosissima, ut præredens, divisa in Partes duas novales, æquales cum pulvillis, numeris adscriptis plantis.

Meridronalis e frigidario plantas Mansuetas excipiat rstate, a frondescentià Quercûs ad forescentiam Colchici.

Vervalis instructa Pariete a plagl boreali, pro plantis seandentibus, et Delicatulis.

Autumanars pro plantis Rarioribus, hyeme obtegenda Musco, et Foliis.

Aquaria effossa pro plantis, cespite vivo circumdata:

Fruviatile aquas (si liceat) motu agitatas vehat.

Lacustre aquam stagnantem, profundam contineat.

Palustre aquam, imbribus inundantem, fundo limoso admittat. Arboreta defendant plagas a procella, nec umbrâ noceant, digesta in Quincunces, Ambulacra, Mapalia, Pergulas.

Occultus sit locus pro Instrumentis, Ligno, Fimo.

Usus: vivæ plantæ a Professore demonstrantur.

Specierum collatio primaria est.

Pretiosa itinera evitantur, dum plures exoticæ occurrunt in urio Horto, quàm facile per to:am Europam spuntanex.

$\mathrm{T}_{4}$ TA-

(") Feræ, qua toto anno intra domum servinda; Mansueix, qu astate exeunt; Cicures, qua forunt clima nosirum bo bornuma. 


\section{T A B U A V. F U L C R A,}

Fig. I1 8. a. Cirrhus,

b. Stipulæ.

c. Glandulæ concave.

II9. Glandulæe pedicellate.

I20. a. Bractea, diversa a foliis b.

I21. a. Spina simplex.

b. - - triplex.

122. Aculeus simplex.

I23. Aculeus triplex, s. Furca.

I24. Opposita folia.

a. Axilla. 


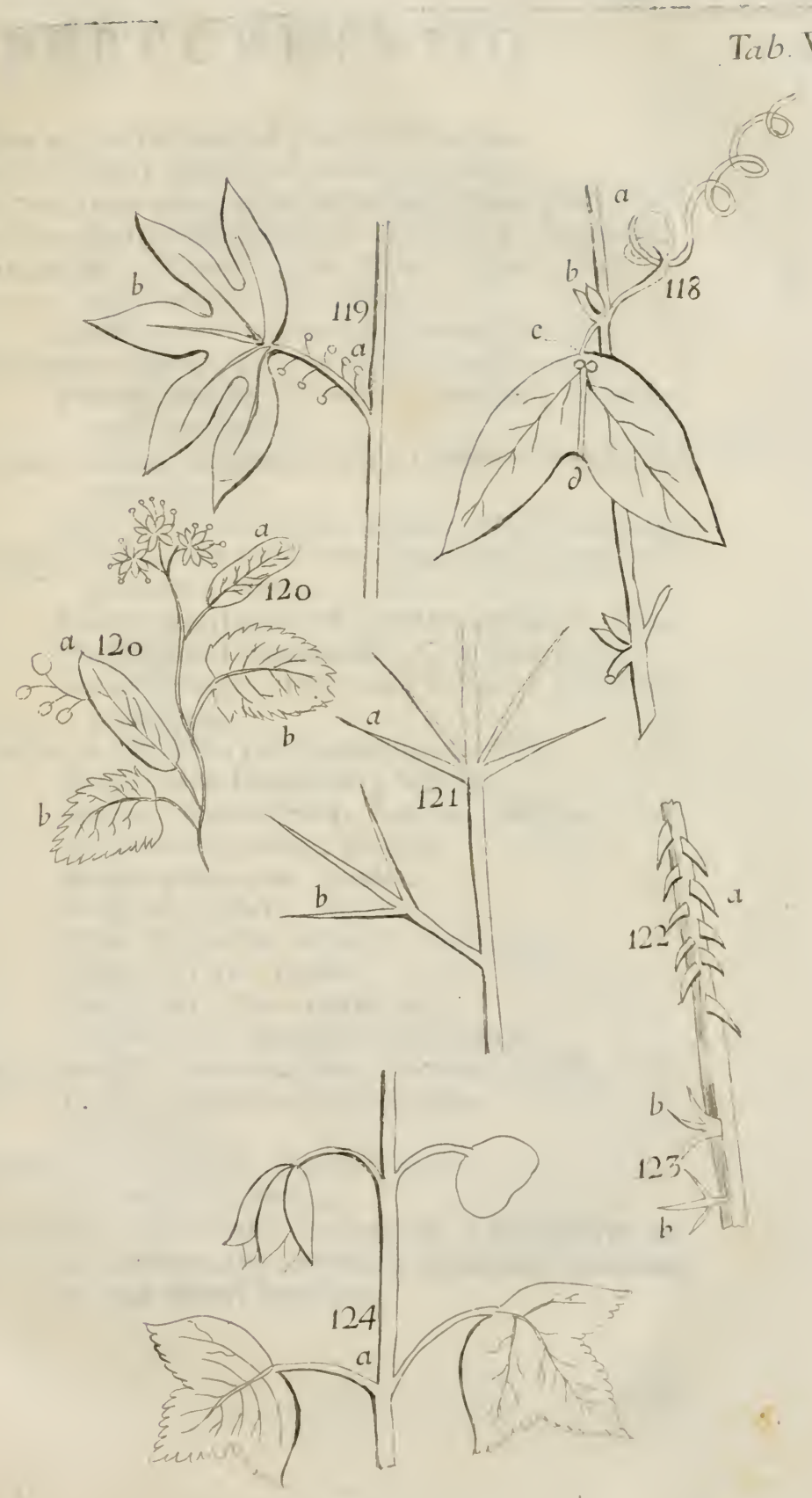





\section{PEREGRINATIO.}

Principium erit mirari omnia, etiam tritissima. Inedium est calamo conmittere visa, et utilia. Finis erit naturam adcuratiùs delineare, quàm alius.

Iter : viæ diurnx dimensio per Territoria, Pagos \&c, Geographica: Montes, juga, Fluvii, Lacus, Urbes. Physica : Cataracte, I ontes: Aciduice.

- Solum minùs frequens, aut proprium loci. Lithologica: Terre, Saxa propria, Lapides varii.

Fodine metalla, Ustrince processus, Irstal'urgire oconomia.

Botanica: Plante indigenæ: loco, tempore, nomire incolarum.

Rariores describend $\curvearrowright$, delineandx, illustrandx. Zoologica: Animalium rariorum Descriptio, Figura, Alimentum, Minres.

Insectorum alimentum, metamorphosis, noxa.

Piscium radii numerandi, Serpentum scuta:

Avium remiges, rectrices; $V$ ermium structura scrutanda.

Oeconomica : Privata fundamentum ejusdem.

Architectura Domorum, Sepium.

Georgica instrumenta, rodus, tempus.

Horticultura qualis, quibus.

Mionotrophrea quæ, quales.

Pratorum cultura, habitus.

Diceta ex quibus primaria, toto anno.

Coquinaria ars, Culina.

Panificium, Poî̀s confectio.

Commercia, Mianuales operationes.

Mores : proprii, conversatione, Sermone, Vestitu.

Festivi nuptiales, sepulchrales.

Superstitiosi.

Antiquitates : Rudera, Tumuli, Rune.

Historica, Fabrilosa.

Palmarium : Plantarum, Animalium, Lapidumque cognitio naturalis, influxus Systematis mundani, et usus generi humano. 
298

\section{T A B U L A V I. R A D I C E S.}

Fig. 125. Bulbus squamosus : Lilii. I26. Bulbus solidus. I27. Bulbus tunicatus transversim dissectus. I28. Tuberosa radix : Filipendula. I29. Fusiformis radix : Dauci. I30. Ramosa radix. I3I. Repens radix. 


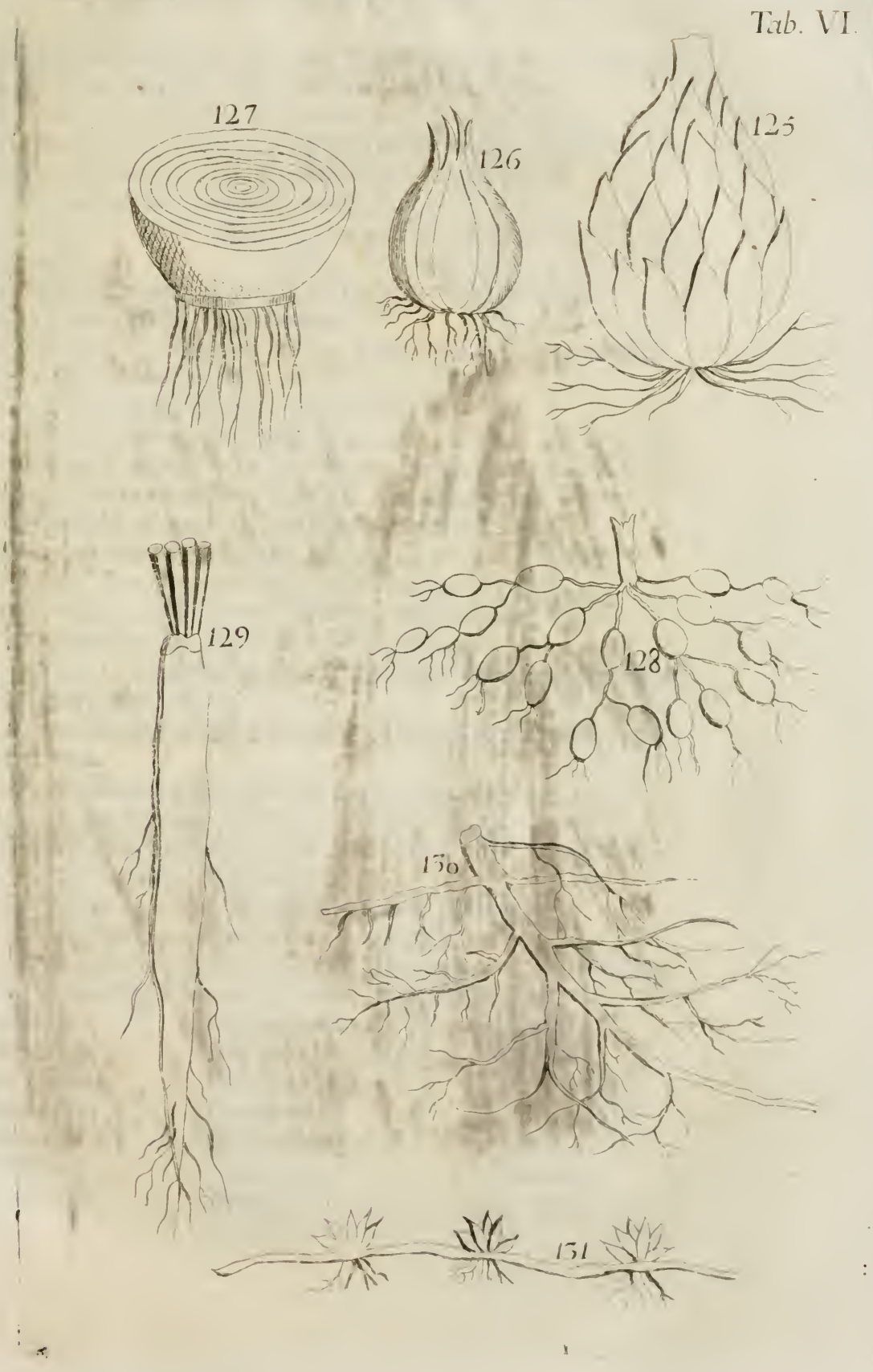




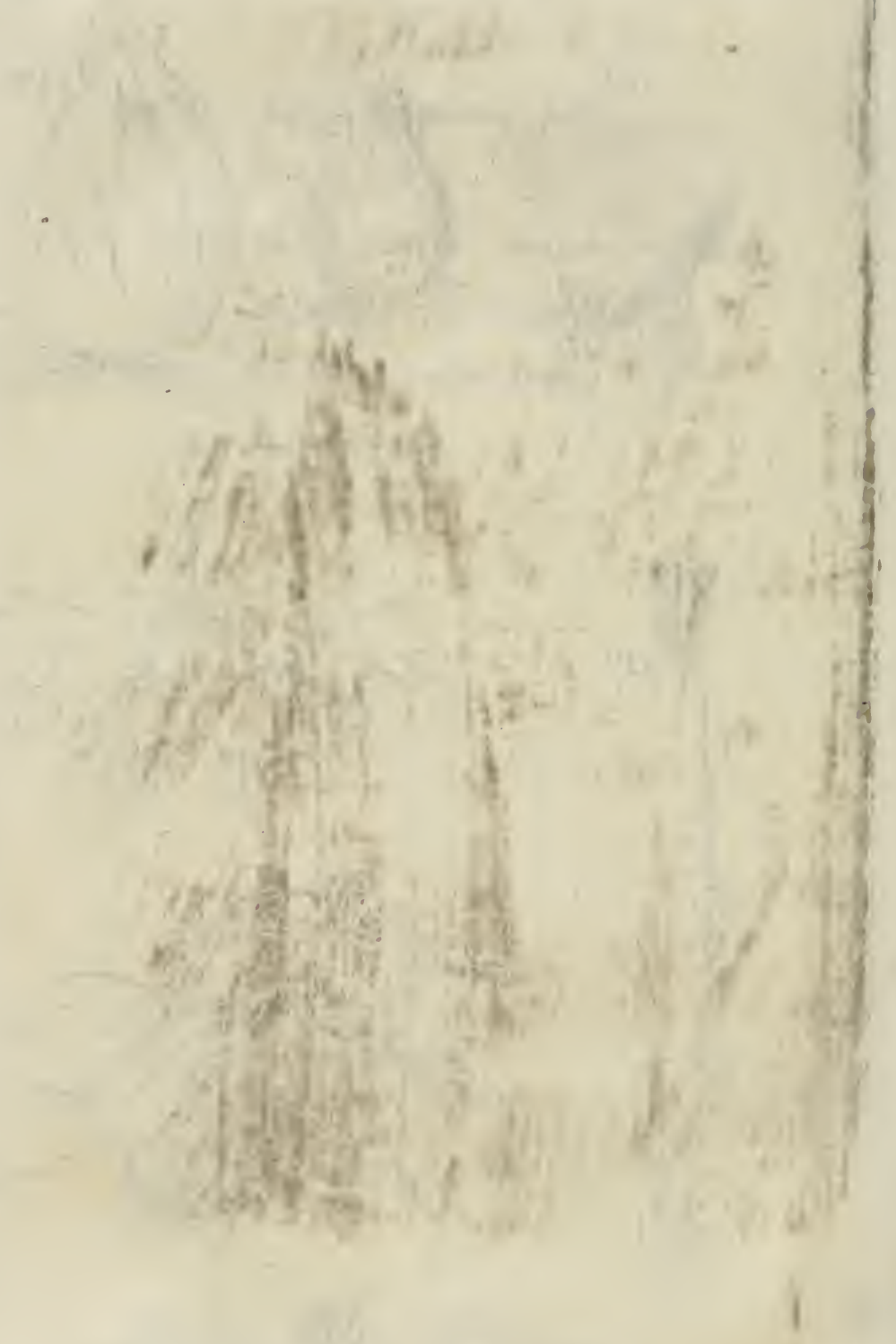




\section{B O T A N I C U S.}

Character Botanici sit Tyroni evidentissimus, ut distinguat veros fiuctores ab eruditis Compilatoribus.

Verus Botanicus ubique Scientiam botanices excolit;

Rudis ostentator nihil in augmentum Scientiæ confert.

I. Systematice plantas suas disponit verus botanicus;

Nec absque ordine easdem enumerat.

2. Fructificutionis principium in theoreticâ dispositione (1 52 ) ą̧noscit;

Nec dispositionem securdùm Herbam (79) immutat.

3. Gener $x$ naturalia ( 162 ) assunit;

Nec Erronea ob speciei notam aberrantem (1 70 ) conficit. 4. Species distinctas tradit;

Nec e Varietatibus falsas fingit (283).

5. Varietates ad species reciucit ( 317$)$;

Nec eas pari passu cum specisbus obambulare sirit.

6. Synonyma præstantissima indagat, et seligit;

$\mathrm{Nec}$ acquiescit in quacunque obviâ nomenclaturà.

7. Differentias characteristicas inquirit;

Nec inania nomina specifica prxponit veris (319).

8. Plantas vagas ad Genera amandare studet;

$\mathrm{Nec}$ rariores obvias fugitivis oculis adspicit.

9. Descriptiones, complectentes differentias essentiales, compendiosè (327) sistit;

Nec naturalissimam structuram (93) oratorio sermone(199) ebuccinat.

Io. Minimas partes (326) attentè scrutatur;

Nec ea, quæ maximè illustrant, flocci facit.

II. Observationibus ubique plantas illustrat;

$\mathrm{Nec}$ in vago nomine acquiescit.

12. Oculis propriis, quæ singularia sunt, cbservat;

Nec sua solùm, ex Auctoribus, compilat.

Magistri in Arte per hanc scalam Floræ ædem adscenderunt;

Hodierni primarii hoc mihi facile largientur : uti
Jussiai,
Gesnerus,
Burmannus,
Guettardus,
Celsius,
Gronovius,
Hallerus,
Rojenus,
Kalmius,
Wachendorffius,
Dalibardus,
Mitchellus,
Browallius,
Claytonius,
Montius,
Gmelinus,
Ludvigius,
Gleditscbius,
Lecòius,
Coldenus,
Aliique. 


\section{TA B ULA VII. PARTES FLORIS.}

Fig. 132. Spatha, Calyx; Narcissi.

133. Spatha. Spadix.

I34. a. Gluma calyx b. Arista.

\$5. a. Umbella universalis. $b$. partialis.

$c$. Involucrum universale. $d$. partiale.

r 36. c. Calyptra ; b. Operculum; $a$. Capitulum.

I37. Amentum.

I38. Strobilus.

г 39. Fungi $a$. Pileus. $b$. Volva. c. Stipes.

I 40. $a$. Receptaculum commune nudum.

141. Receptaculum commune paleis imbricatum.

I42. Corolla monopetale $a$. Tubus. b. Limbus.

143. Flos $a$. Germen. b. Stylus. c. Stigma $d$. Filamenta. e. Antherx. $f$. Petala.

544. Corolle polypetale. $a$. Ungues; $b$. Laminx.

145. Nectarium campanulatum in Narcisso.

I46. Nectaria cornuta in Aconito.

147. Nectarium cornutum in calyce Tropeoli.

148. Nectaria in Parnassiâ. 


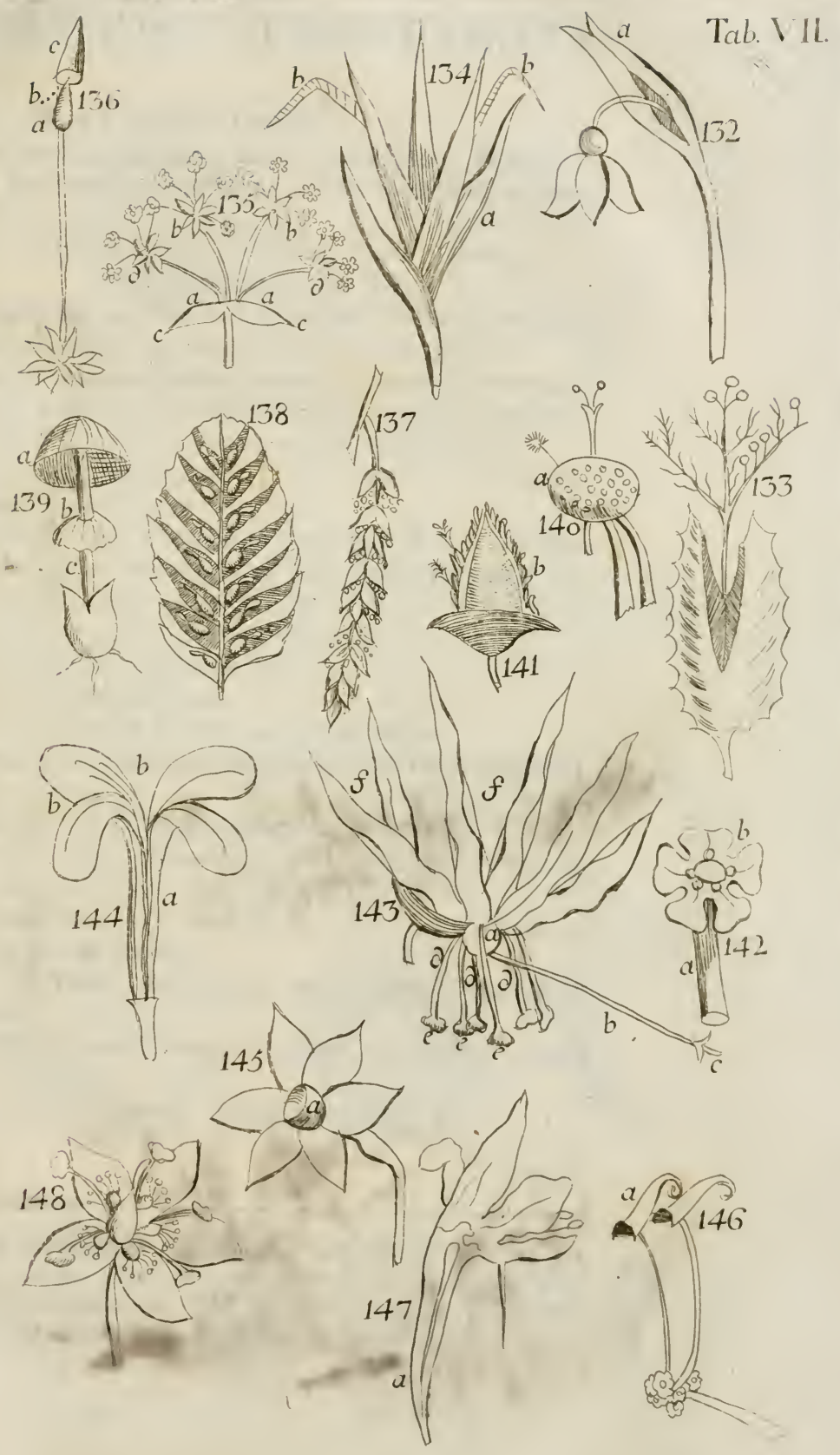




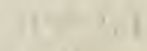

H:

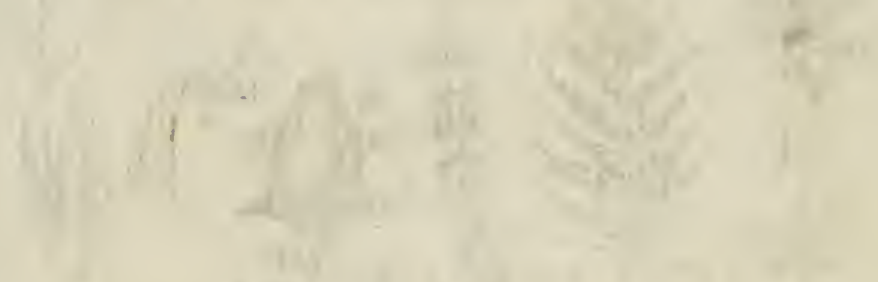

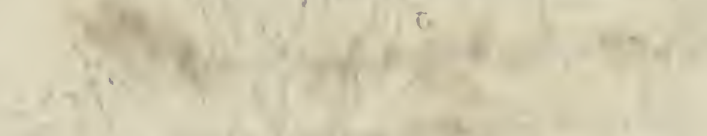

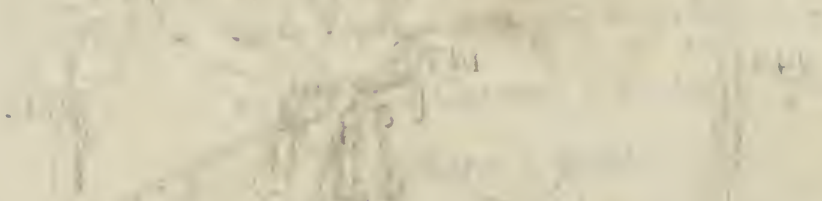

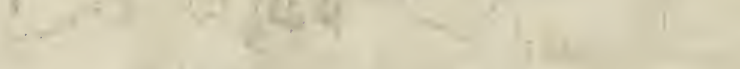

$$
x-1=
$$

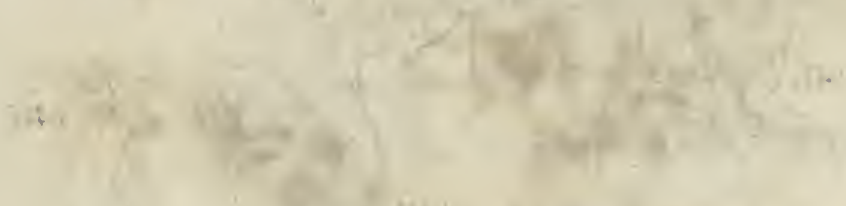




\section{METAMORPHOSIS VEGETABILIS.}

Radix deorsum, Herbx sursum tendit.

Herba omnis est processus substantia medullaris Radicis.

Flos terminat, Focundatio separat, Semen incipit omrem plantam.

Terminus plante idem, vel vita continuata in Gemmâ, vel propagata in Flore.

Gemme fines, Semina initia plantæe defendunt a climatis duritie. Gemma est Hybernaculum herbæ, Semen ejus capsella.

Gemma conservat embryones intra squamas, Semen intra tunicas. Gemmipare non minùs sunt perennes herbæ, quàm Arbores. Meridionales arbores nequeunt esse boreales ob gemmas.

Perennis planta gaudet duplici flore, altero antecessore triumphante; altero successore occulto.

Gemma aut gemmulis, aut fioribus, aut utrisque gravida est. Plumulam seminis sxpius terminat aut flos, aut gemma. *

Principium florum, et foliorum idern est.

Principium gemmarum, et foliorum idem est.

Gemma constat foliorum rudimentis.

Stipule sunt foliorum appendices.

Perianthium fit ex connatis foliorum rudimentis.

Derivato nutrimento ad squamas amenti, destructis flosculis, mutantur in Folia.

Derivato nutrimento ad flosculos amenti, funt folia Calyces. Luxurians vegetatio folia e floribus continuando producit. IHacra vegetatio flores e foliis terminando producit.

Gemmæ vix frondescunt, nisi defoliatâ arbore, vel foliis ex climate mutationem passis.

Gemmæ sæpius destruunt sequentis anni fores, exfoliatâ æstate arbore.

Gemmæ annuo spatio preccciores evadunt, exfoliatâ æstate arbore.

Flos ex gemmâ, annuo spatio, foliis præcocior est.

* Amygdala sata in caldario producunt Folia alterna; at spatio determinato, supra radicem, in gibbosiore caule conterta, loco determinato gernme, ubi citatior propu!sio superavis moram, et revocavit futurum annum in prasentera. 
302

\section{T A B U L A V I I I.}

\section{PARTES FRUCTIFICATIONIS.}

Fig. I 49.a. Perianthium, b. Germen, c. Stylus, d. Stigma. e. Filamenta, $f$. Antheræ debiscentes, $g$. Antheræ integre.

I 50.a. Filamentum, $b$. Anthera.

I 1 1.a. Pollen microscopio visum, $b$. Halitus elasticus. I 52.a. Germen, $b$. Stylus, $c$. Stigma.

153. Folliculus, a. receftaculum seminum.

154. Legumen, a. a. sutura superior semina annectens.

I55. Siliqua, a.b. receptaculi margo uterque.

c. Valvula siliqua.

I 56. Pomum, a. capsula, b. inclusa.

I57. a. Drupa, $b$ Nucleo.

I58. Bicca.

I 59. Capsula apice debiscens.

160. Capsulæe a.Valvula.b. Dissepimentum, c. Columella, d. Receptaculum.

I6I. Capsula longitudinaliter dissecta, ut seminum receptaculum in con
162. a. Pappus pilosus.

b. Pappus plumosus.

c. Semen.

d. Stipes pappi. 


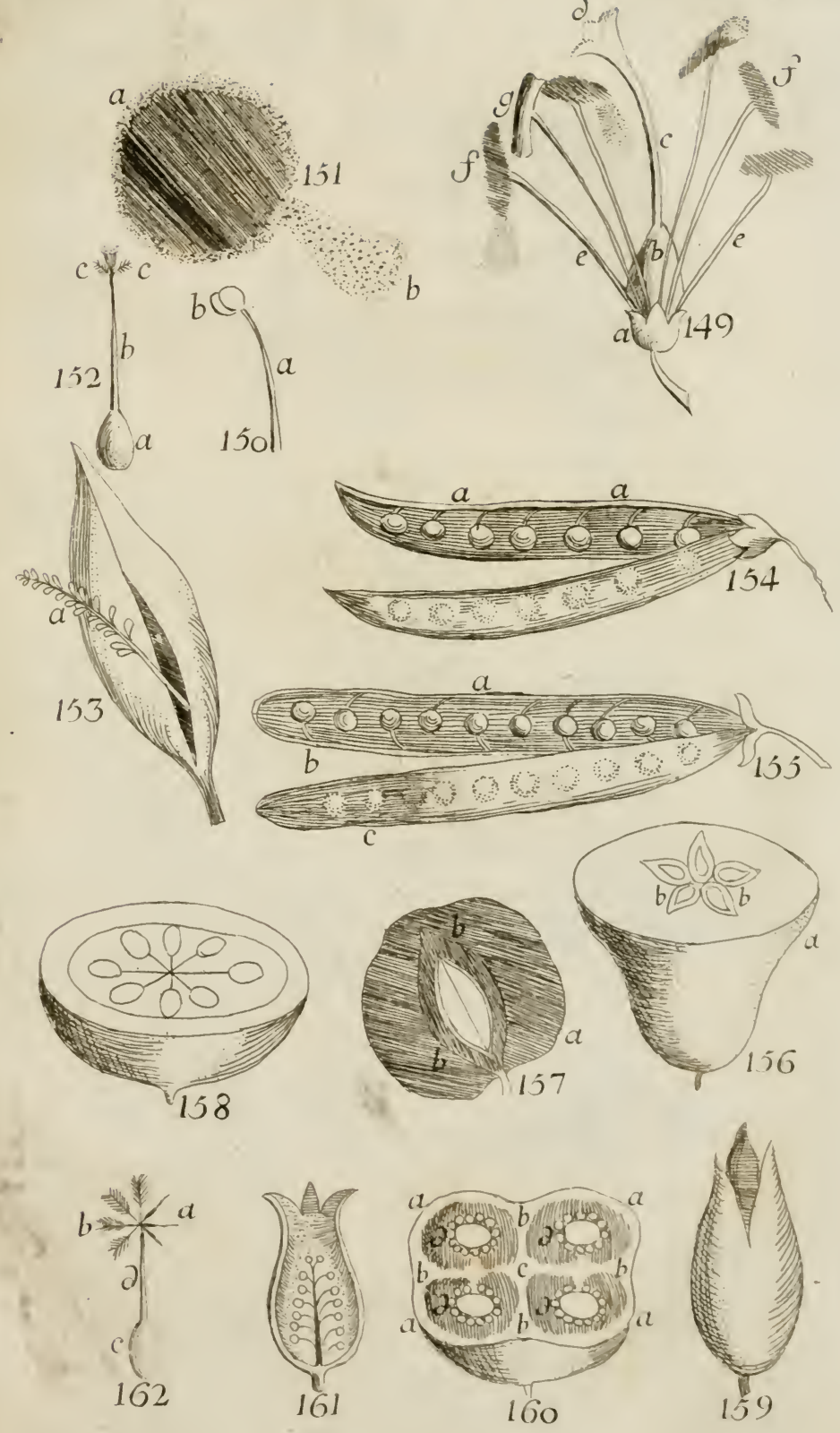

151

$b$

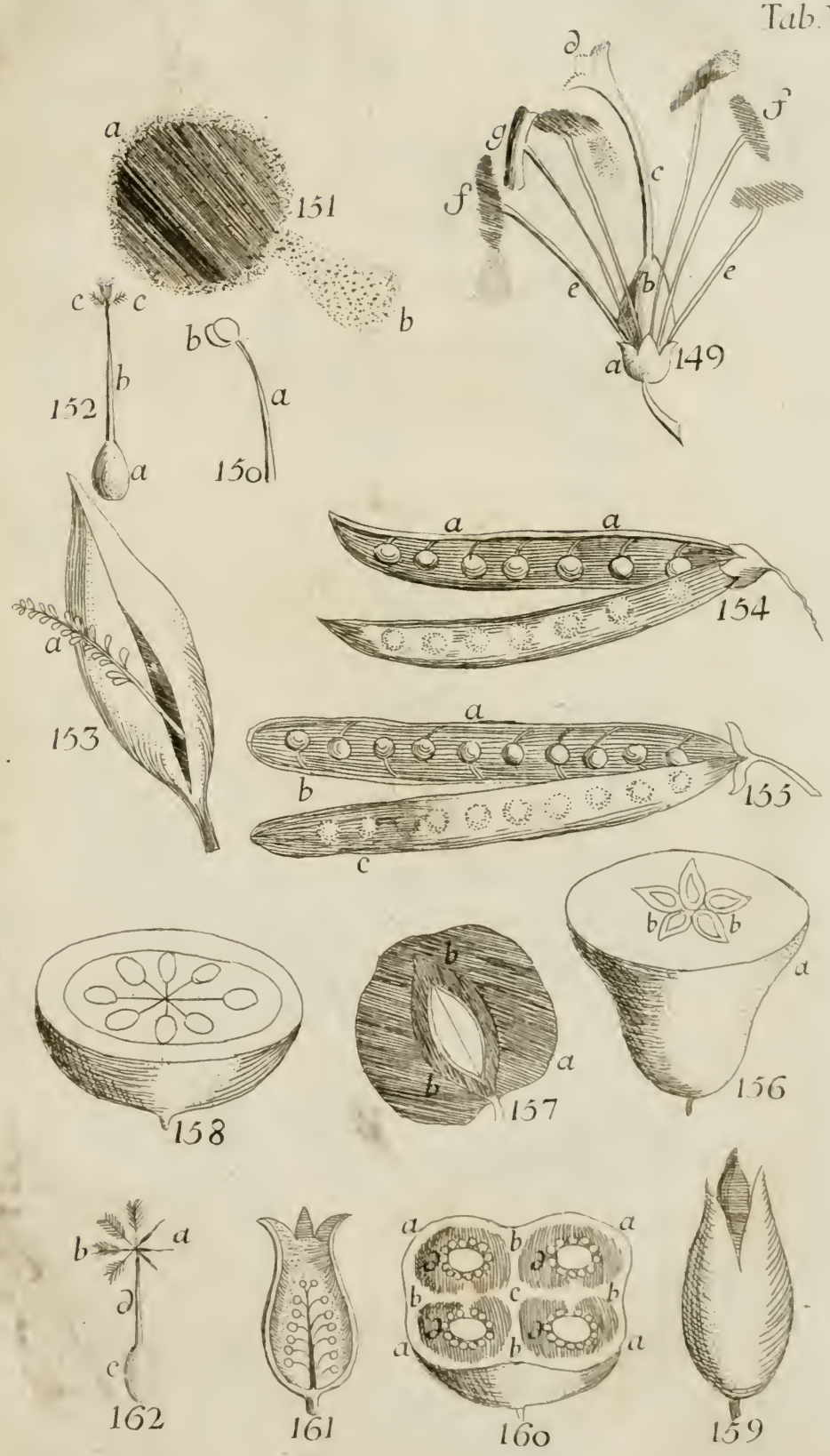

Tab. V'III. 


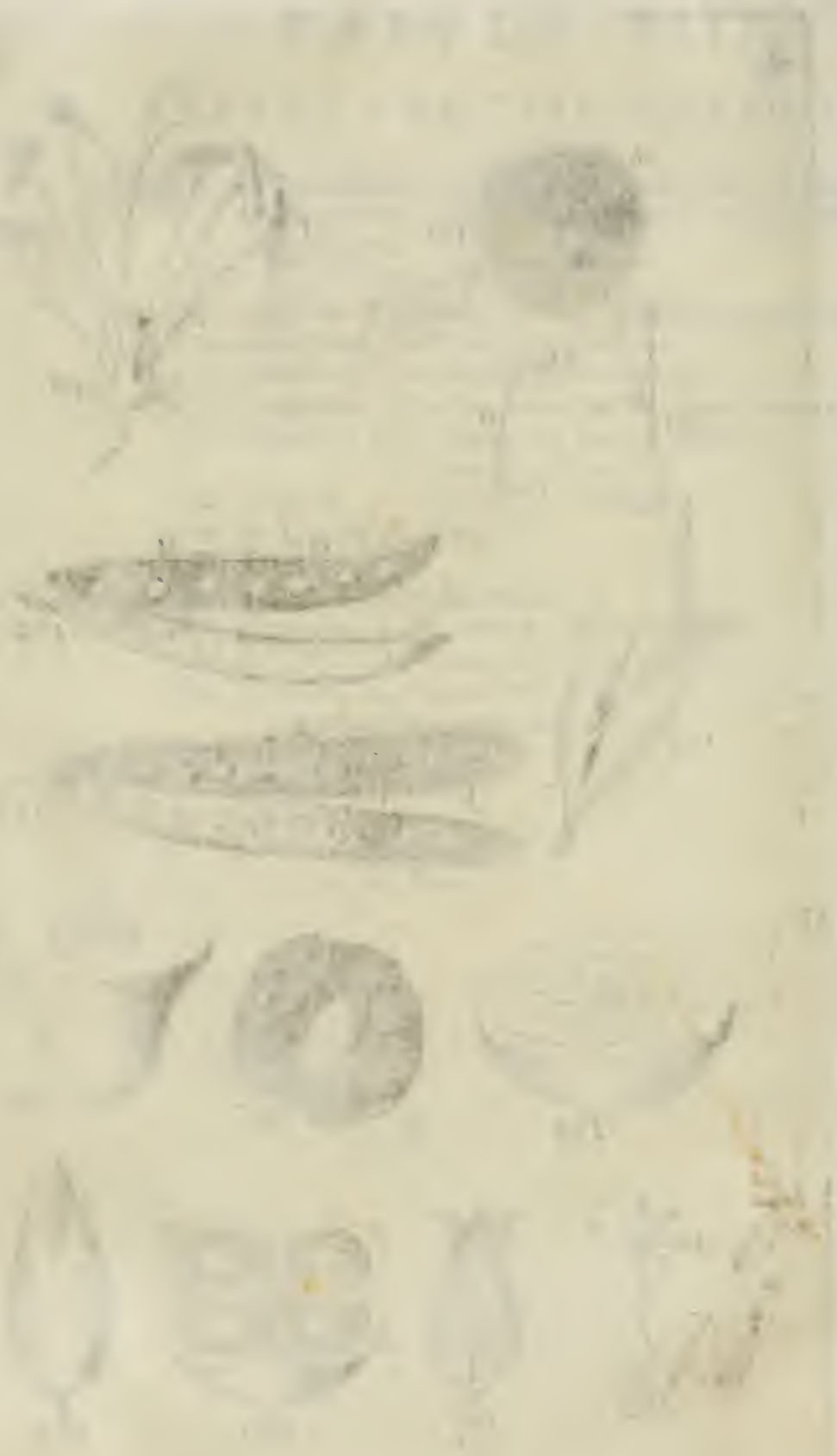


48
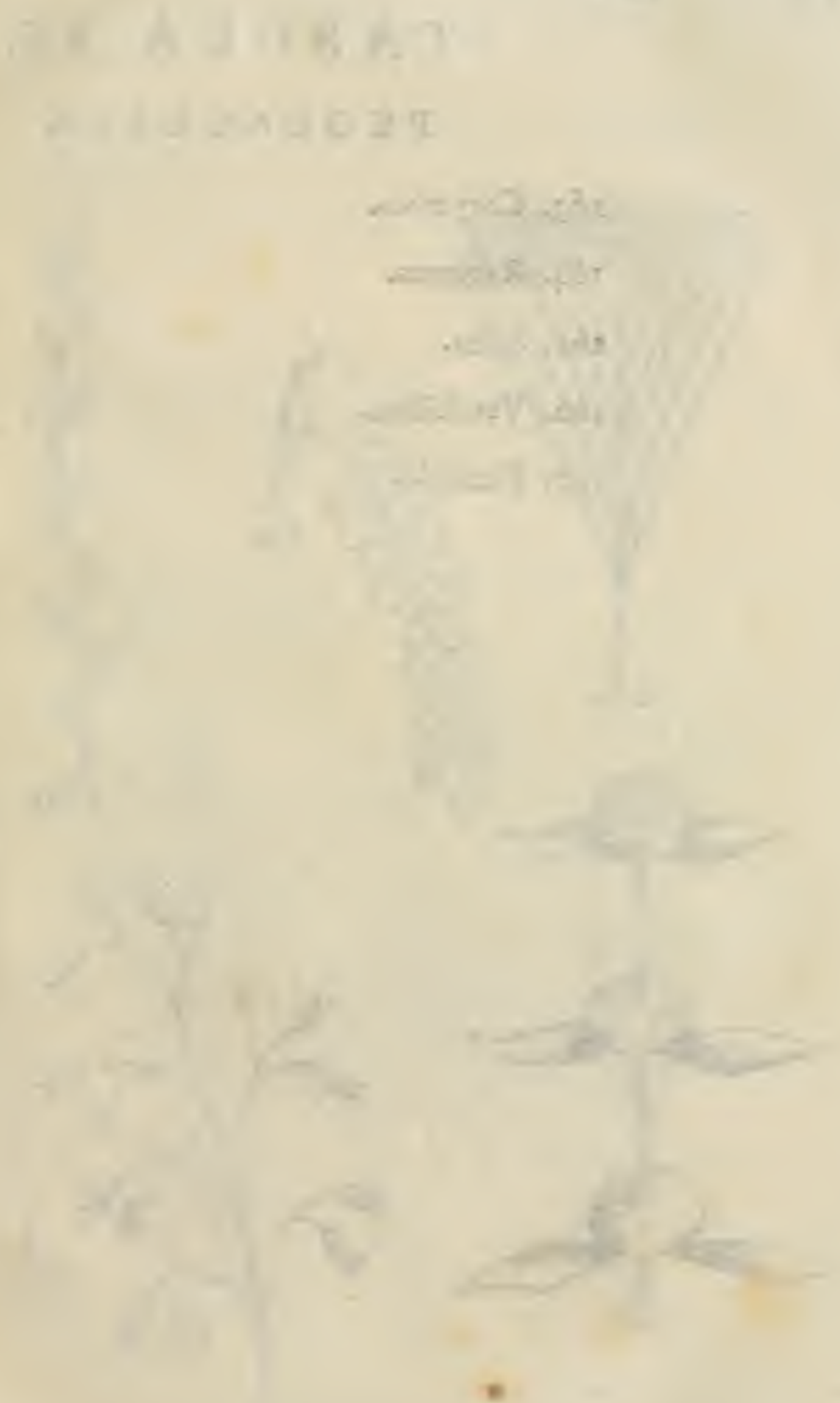

7. 
304

\section{T A BULA IX. PEDUNCULUS.}

x63. Corymbus.

164. Racemus.

165. Spica.

ฐ66. Verticillus.

I67. Panicula, 
Tal 1X.

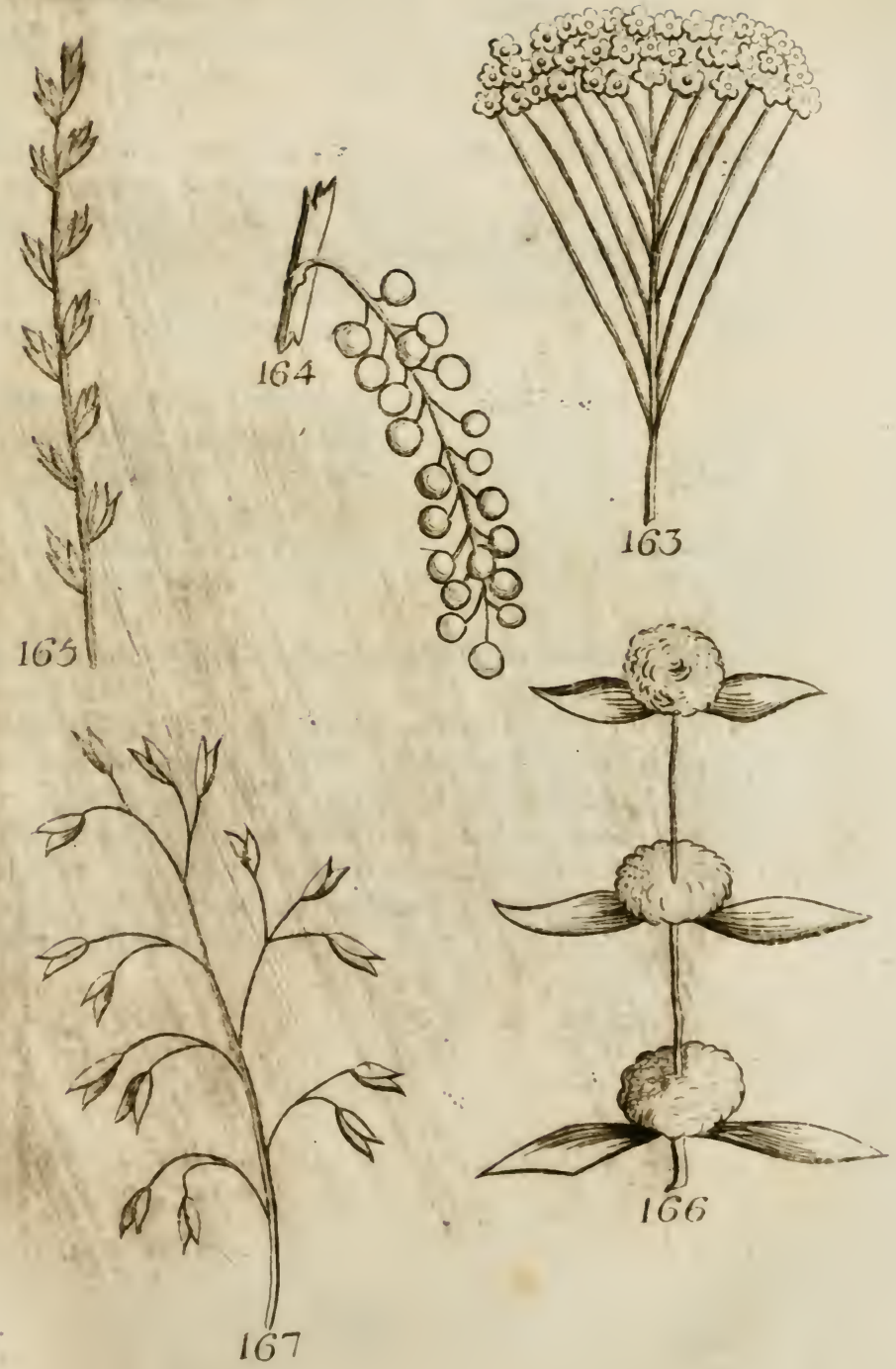





\section{A D D E N A.}

Plenitucio.

XERANTHENII impletio por Paleas propria est, et singularis, ut in fasione.

Palex disci elongatæ, coloratæ, expansæ, facie, et structurâ squamarum calycis radium mentientium.

Flosculi naturalibus vix majores, adeoque paleis breviores.

Radii feminei steriles! Stylo patente, Jongitudine palearum. Stigmate simplici.

Disci bermaphroditi Stylo breviore, Stigmate bifido. Obs. Floris simplicis flosculi, calyci proximi, hermaphroditi sunt ; singulare itaque, quòd Plenus flos radium femineum assumat.

RIBES inerme, floribus oblongis. Hort. cliff. 209.

Germen excluditur in pleno flore.

Calyx quinquepartitus ad basim, reflexo-patens, coloratus: laciniis linearibus.

Corolle loco series triplex petalorum, calyci simillima. Staminum, Pistillorumque loco Conus acutus quinquefidus.

HYACINTHUS, paniculà comosâ, sanesius, Cohmn ecphr. 2.p. I0. $t$. I 2 .

Spica in Paniculam conglomeratam effusa.

Siylis, nec his satis evidentibus, videntur omnes Flores constare, absque Corollâ, Staminibus, Germine, aut alio rudimento florum, sxpe 20 in eodem pedunculo. Squame minimæ, alternæ, subjectæ cuivis stylo; ex singulâ squamâ videtur novus pedicellus prognasci; quid itaque monstrosius? 
306

\section{T A B U L A $X$. F O L I A T I O.}

Foliis transversim dissectis

x. Convolutum.

2. Involutum.

3. Revolutum.

4. Conduplicatum.

5. Equitans.

6. Imbricatum.

7. Obvolutuma

8. Plicatum.

9. Convoluta,

I0. Involuta opposita.

II. . . alterna.

12. Revoluta opposita.

13. Equitantia ancipitia.

I4. . . . triquetra.

M ENS URA.

P. Parisini $P e d i s \frac{T}{4}$

A. Anglicani Pedis $\frac{T}{4}$

S. Suecici Pedis $\frac{\pi}{4}$ 

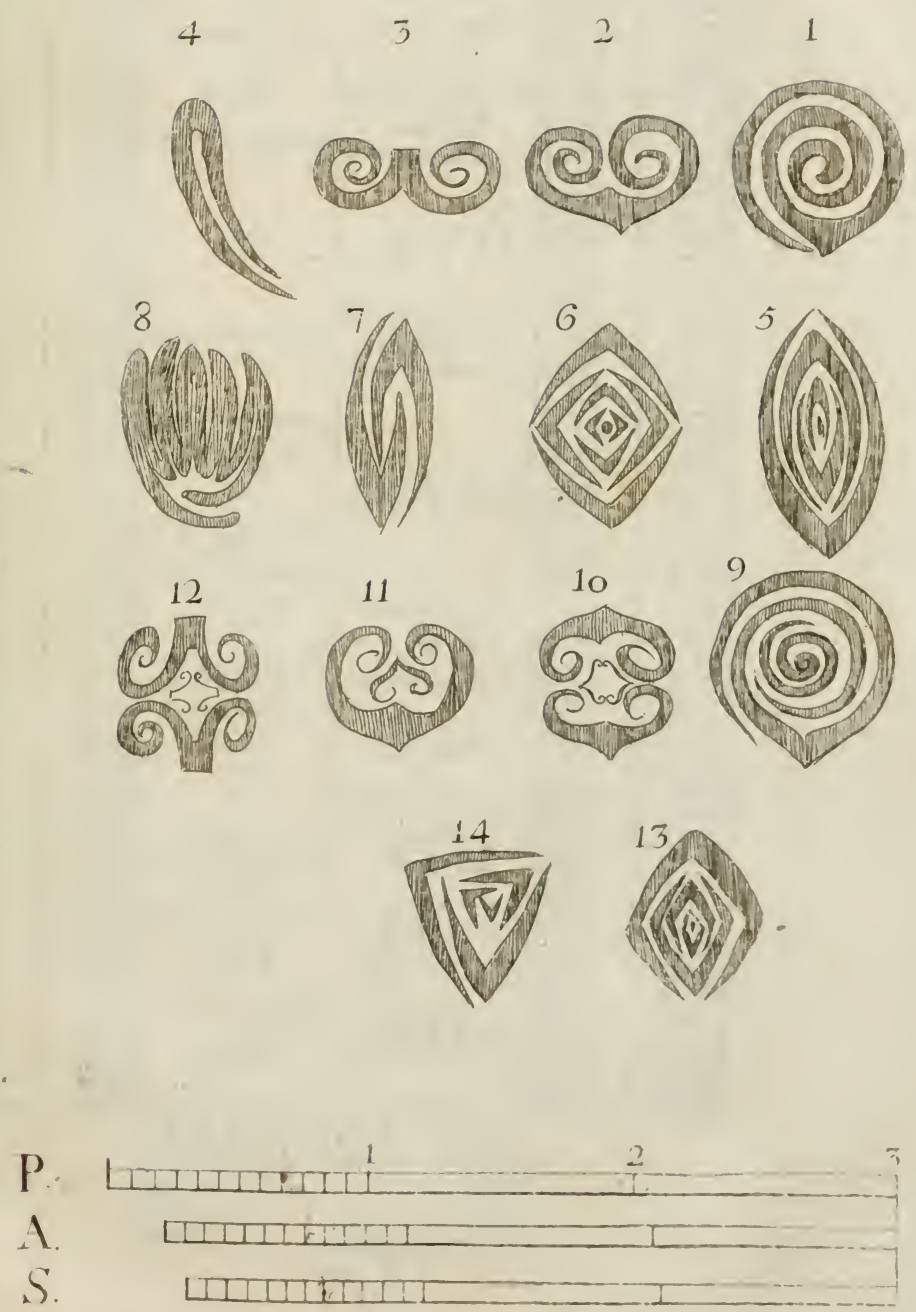


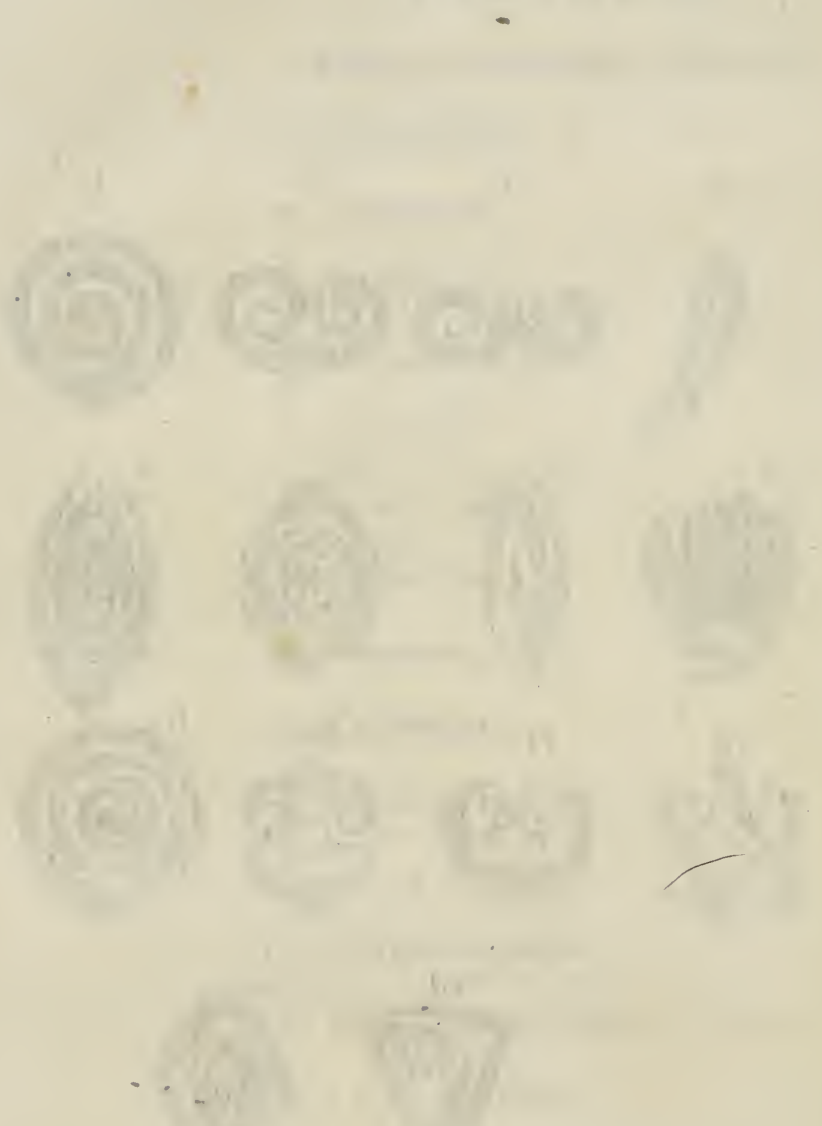


T A B U L A X I.

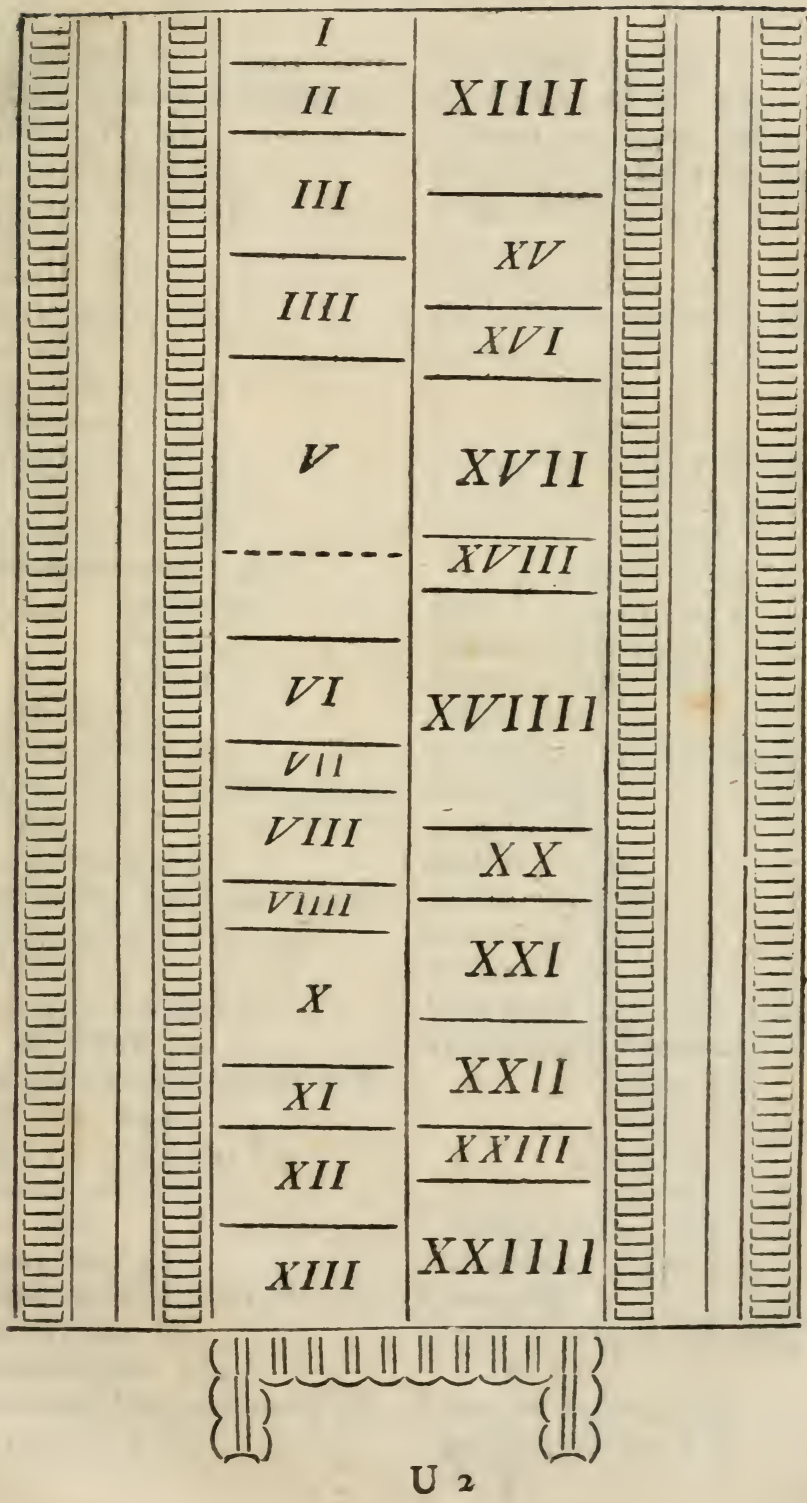


$1 x+15=: T$

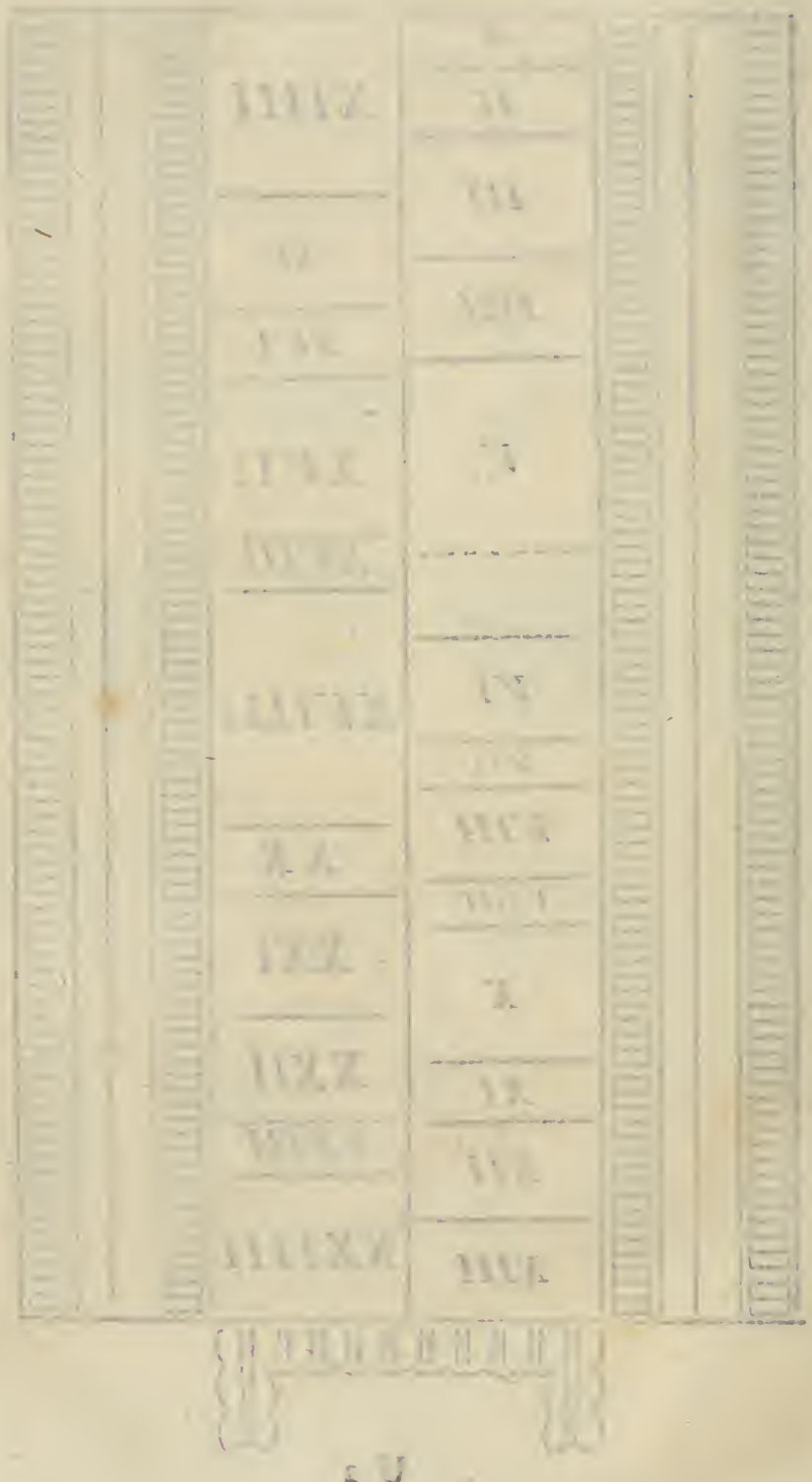

$$
\text { (i) } \begin{array}{r}
\frac{1}{4}+1 / 1 \\
=0
\end{array}
$$




\section{T E R M I N I.}

A

Abortiens flos I 34 .

Amentum 52. 76.

Abruptè pinnatum folium 47. Amplexicaule fol. 49 .

Acalyces W. 25.

Acaulis herba 233 .

Acerosum folium 42. 219.

Acidus 285.

Acinaciforme fol. 46.224.

Anceps caulis fol. 46 .

Androgyna planta 93.94 .

Angiosperma H. I 35.19 .

Angulatum fol. 233 .

Angulosum fol. 233.

Acotyledones 102.

$A=r e 285$.

Aculeatum fol. 45 .

Aculeus 50. Iro.

Acuminatum fol. 44 .

Acutum fol. 44.233.

Adpressum fol. ${ }_{4} 8$.

Adscendens caudex 38.

.... caulis 40 .

- . flos 60.

- . folium 49.

Adversum fol. 49.

Æqualis corolla 223 .

Equinoctiales solares flores

$$
273 .
$$

Afora pericarpia 22.

Anisostemonopetale W. 25 .

Anomalus Flos T. I 23.

- - plant.23.

- - calyc. 24.

Anomojodiperianthe W. 25 .

Anthera 53. 56. 90.92.

A petalus flos 58.76 .

- . plant.19.20.21.25.

Apex T. I I3. I 34.

Aqueus color 243 .

Aquosus sapor 285.

Arbor 37.139 .

Arbustiva (39. ord.nat.) 3 I.

Aggregatæ(1 8.ord nat.) 28.25 .

Aggregatus flos 26. I 34 .

Agri 268.

Ala papilionaceæ 135 .

... seminum 54.

Alati pedunculi, s. caules 2 I8. . . . bulbus 50.

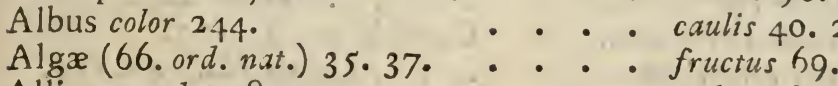

Alliaceus odor 285 .

Alpes 266.

Alterna folia 48.

Alternatim pinnatum fol. 47.

Amarus sapor 285.

Ambrosius odor 284 .

Arena 269.

Argilla 269.

Arillus 54:70.

Arista 52. 233.

Aromatica 285.

Arrectum fol. 2 i 9 .

Articulatè pinnatum fol. 47 .

Articulatum folium 47 .

Amentacex (16. ord. nat.) 28. Asperum folium 219.

$$
23.24 \cdot 25 \text {. }
$$

Assurgens folium 219.

$$
\mathrm{U}_{3}
$$


Ater color 244.

Attenuati pedunculi 233.

Auctus caly: 62.

Auleum fioris 92.

Autumnales 276.

Avenia folia 219.233.

Axillare folium 48.

-. pedunculus 4I.

\section{B}

Bacciferæ 19. 20. 2 I.

Barba corollæx 135 .

Barbatus fios Riv. 134 .

Bicapsulare peric. 68.

Bicornes (24. brd. nat.) 30.

Biferæ plante 277.

Bifidum fol. 43 .

- . calyx 63 .

Biforus peduncul. $4 \mathrm{I}$.

Biforum pericarp. 22.

Bigeminatum folium 47.

Bilobum fol. 43.

Biloculare pericarpium 69.

- . . semen 70.

Bina folia 219.

- peduncul. 222.

Binatum folium 47.

Bipartitum fol. 44 .

Bipinnatum fol. 47.

Biternatum fol. 47 .

Bivalve pericarp. 69.

Bivasculares 19.

Brachialis mensur. 262.

Brachiatus caulis 40.

Bractea 50.57 .

Eracteate monocotyl. 20.

Brumales berbe 276 .

Bulbosæ 27.

Bulbosis affines 27.

Bulbus 50 .

Bullata folia 217.243.
Caducus (calyx) 63.65.

Cæruleo-purpureus color 244 .

Cæruleus color 244.

Calamariæ (13. ord.nat.) 28.

Calcar Corolle 223.

Calide plante 277.

Calix T. I I 3 .

Calycant kema (40. ord.nat.) $3 \mathrm{I}$.

Calycifiore 25.

Calycini W. I 6.

Calyculatus calyx 62 .

Calyptra musc. 52.

- semin. II3. 70.

Calyptrati musci 27.

Calyx 52.56.92.

Calycem abire in fructum $T$.

124.

Campanacei (30.ord.nat.) $3 \mathrm{I}$.

Campaniformes 23.

Campanulata Corolla 52.

Campi 267.

Canaliculatum fol. 46 .

Candelares (62. ord. nat.) 35 .

Capillares berbe 19.20. 2 I.

Capillaris pappus 70.

Capillaceus 262.

Capitati (2 1.b.ord.nat.) compositi 29.

. flos Raj. I 34. •

Capitulum 4I.

- . - musci 223.

Capsula 53. T. I 13.

- : staminis Malpigh. I34.

Carina papilionacee I 35 .

Carinatum fol. 46.

Carnosum fol. 46.

Cartilagineum fol. 44 .

Caryophyllex (42. ord. nat.) 3 I. 23.

Caudex adscendens 38 . descendens 38 . 
Caulescens pl. 233.

Caulinus pedunc. $4 \mathrm{I}$.

Caulis 39.

Cerealia semina 279.

Cernuus pedunculus $22 \mathrm{I}$.

Cespitosa planta 2 I 6.

Cespitosæ paludes 266.

Character essentialis I 28.

... factitius I 30.129.

-. habitualis I I 8 . I 28 .

... naturalis I29.130. I3 I. I 35.

Cicboraceus flos V. I 3 .

Ciliatum fol. 44 .

Cinereus color 244.

Circinalia fol. ro6.

Cirrhosum fol. 44.

Cirrhus 50.

Classis r oo. r or. I 36 . I 37. - naturalis 27.

Coadunatæ (1 2. ord. nat.) 28. - . fol. 2 rg.

Coarctatus pedunculus

$$
233 .
$$

Coccineus color 244.

Coloratum fol. 452 I I.

Columella capsule 53.

Columniferæ $(34 \cdot 0 . n$. $) 31.25$.

Coma 220.

Commune Receptaculum 54.

Completus flos V aill. 34 .

Composita Umbella 54 .

- . (21.ord.n.zt.)29.25.

- fol. 47.233.

- flos 76. 77.223.19.

Compressum fol. 46 .

Concavum fol. 45 .

Conceptaculum 53 .

Conduplicatum fol. 106.

Conferta folia 48.233.

Conglobatus fios 134.222.
Conglomerati fores 222 .

Congregata 25.

Coniferi (I 5 . ord. nat.) 28.

Conjugatum fol. 47.

Connata folia 49 .

Connivens coroilla 223 .

Contorsio 104 :

Contorti (29. ord.nat.) 3 r.

- . fructus 222.

Contrarium dissepimenium

I 35 .

Convexum folium 46.

Convolutum folium 105 .

Conus T. I I 3. I 34.

Cor vegetabil. 93.

Corculum semin. 54.7 r.

Cordatum fol. 43 .

Corolla 52.56.57.92.

Coronariæ (9. ord. nat.) 28.

Coronatrices (Method. calyc.) 24 .

Coronula seminis 54 .

Cortex 37.

Corydales (28. ord. nat.) 30.

21. Corymbiferæ (2I.c.ord. nat.)

I 9.2 I. 26.

Corymbus $4 \mathrm{I}$.

Cotyledon 54.49 .

Crenatum fol. 44 .

Creta 270.

Crispum fol. 45.217.

Croceus color 24.

Cruciformis coroll. 53.23 .

Cryptanthe W. 25.

Cryptantherce Roy. 25.

Cryptogamia 25.

Cubitalis (mensura) 263.

Cucullatum fol. 219.

Cucurbitacex (45.ord.nat.) 32.

Culminere (26. ord.nat.) 32 .

Culmifere 19.

Culinus 40.

Culta Terra 269.

U 4 
Cuneiforme fol. $4^{2}$.

Cuspidatum fol. 42 .

Cyathiformis coroll. 223 .

Cylinitianthere 25.

Cylindrobasiostemones 25 .

Cyma 55. 78.79 .

Cymose (63. ord. nat.) 35 .

Cymosus fios 76 .

CynarocephaliV aill. 26.

ह

Decaphyllus calyx 62 .

Decemfidus calyx 63 .

Decemloculare pericarp. 60.

Deciduus 63.65 .

Declinatum fol. 2 I 9 .

Decompositura fol. 47 .

. . flos 223 .

Decumbens fios óo.

- . fol. 49.

Decursivè pinnation fol. 47 .

Decussata fulu 2 I 9 .

Defoliatio 275 .

Dehiscens, 67.

Delioides fol. 43 .

Demersum fol. +9 .

Denominatio I 38 .

Dentatum fol. 4.4 .

Denudatx (7. ord. nat.) 28.

Dependers fol. 49 .

Depressum fol. 46 .

Descendens caudex 38 .

Descriptio 256.

Dextrorsum volubilis 39.103 .

Diadelphia 25.

Diandria 24.

Diangria 20.

Dichotomus caulis 40 .

Dicotyledones 102.

Didynamia 25 .

Difiormia folia 246 .

- flos 134.22.
Diffusus caulis 40.

D. . panicula 222.

Digitatum folium 90.

Digonis caul. 40.

Dimacrostemones W. 25 .

Dioecia 22.

Dipetali 58.22.

Diphyllus 2 I 8 .

- calyo 62.

Diploytanthre W. 25.

Diplosantberce W. 25 .

Diplostemones H. 25 .

Dipsacei V. 26.

Discoidece 2 I.

Discus 223.

Disperma 70.

Dissectum fol. 2 I 9.

- valvis contr. T. ז35.69.

- valvis parall. T. I35.69.

Distemonopleatatibere W. 25 .

Disticha folia 49 .

- . spica $4 \mathrm{I}$.

- : caulis 24.

Divaricati 233.

Dodecandria 24.

Dodrantalis mensura 262 .

Dolabriforme 46.

Drupa 54.

Drupaceæ (38. ord. nat.) $3 \mathrm{I}$.

Dulcia 285 .

Dumosæ (19. ord. nat.) 29.

Dunlicato-pinnatuin jol. 47.

Duplicato-ternatum fol. 47.

Duplicatus (calyx.) 24 .

- - flos 80.

1 chinatum pericarp. 224.

Elsutherantherce W. 25 .

Efflorescentia 272.

Echinatus fung. 27.

Ellipticum fol. 42 .

Emarginatum fol. 44 . 
Enervia fol. $2: 9.233$.

Enneandria 24.

Enodis culmus. 40.

Ensatæ (5. ord. nat.) 27.

Ensiforme fol. 46 .

Epidermis 37.

Epiplyyllosperme 25.

Equitantia fol. 106.

Erecta anthera 223.

Erectum fol. 49.

- - caulis 233.

- - flos 60.

Erosum fol. 44 .

Essentialis character I 28 . I 30.

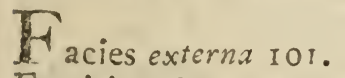

Factitius character I 29. I 30.

Farctum fol. 233 .

Fasciata planta 216.

Fasciculus. $+\mathrm{I}$.

Fastigiati pedunc. 22 I.

Faux corolla 223.

Femina planta 93.94.

Femineus fos. 93.

Ferrugineus color. 244.

Fibrosa Radix. 38.

Filamentosi $\mathrm{pl} .23$.

Filamentum 53. 56.92.

Filices (64. ord. nat.) $35 \cdot 37$.

Fimeta 269.

Fissum fol. 43.233 .

Flaccidus ped. 22 I. 233.

Flammelis color 244.

Flavus color. 244.

Flexuosus caulis. 39.

- - pedunc.222.

Floribundi (calycin. meth.) 24 .

Florale fol. 57.

Flos. 55.56 .90$.

Flosculosi. 77. 23 .

Flosculus. 76.

Fluvii. 265.
Folia 39. 93.

- floralia. 57.

Foliatio 105.

Foliatus caulis. 39.

Folliculi I I .

Fontes. 265.

Fossæ. 266.

Fragrantia 284.

Frigidæ plantæ. 277.

Frequens planta. 2 I 6.

Frondescenia. 271.

Frondosus prolifer flos. $8 \mathrm{I}$.

Frons 42.

Fructescentia 275.

Fructificatio 52. 56.

Fructiferæ Roy. 25.

Fructus. 55. 56.

- - carnosus. II3.

Frumenta 21.

Frutex. 37.

Frutices. 18.

Fulcra. 39. 50.

Fulcratus caulis. 40.

Fulvus color. 244.

Fungi. 35.21 .25$.

Furce. i 10.

Fuscus color. 244.

Fusiformis rad. 38 .

Talea corollce Riv. I35.

Gemma. 37. 90.

Gemmatio. 104.

Genera. I00. I о I.

Generatio. 90. 9I.

Geniculum. 223.

Genitalia fem. $6 \mathrm{r}$.

- - masculina.90.

Genitura. 90.

GEnus. 100. I0I. 135 .

- - subaltermum. R. I35.

- - summum. R. 135 .

Germen. 53. 56.92. 
Germinatio. 270.

Gibbum fol. 46 .

Gilvus color. 244.

Glaber caulis. 40.

Glabrum fol. 45.233 .

Glandulæe 50.

Glandulatio. r ro.

Glandulosum fol. 2 I 9.

Gluma. 52.

Glumosus aggregat. flos. 77 .

Glumosæ. W. 25.24.

Giutinosum. 236.

Gramina (I 4.ord. nat.)28. 37 .

$$
21.25 \text {. }
$$

Graminibus affinia 25.

Graminifoliæ 20. 2 I.

Graveolentia. 285.

Gymnomonospermæ. 20.2 I.

Gymnopolyspermæ. I9. 20.

Gymnospermi. 58. I 34. I9.

$$
21.25 .
$$

Gymnotetraspermi. 2 I.

Gynandria. 25 .

\section{FI abitatio plantar. 254 .}

Habitualischaracter. I I 8. I 28.

Habitus. I 0 I.

Hami. Io9.

Hastatum fol. 43 .

Heptandria. 24.

Herbacea planta. 37.233 .

Herbarium. 7 .

Hermaphrodita planta.93.94.

Hermaphroditus flos. 93.

Hesperideæ (4I. ord. nat.) 3 I. Hexandria. 24.

Hexafora pericarp. Camell. 22 .

Hexapetali 58.22. 64.

Hexaphyllus cal. 62 .

Hexapoda (mensura.) 262.

Hilum. 54.

Hircinus odor. 285.
Hispidum fol. 45. - - caulis 40.

Homnjodiperianthre. W. 25.

Hordi fructus. 213.

Horizontale fol. 49.

- - rad. 38 .

Humus. 269.

Hyalinus color. 243.

Hybernaculum. 39. 50.

Hybrida plant. 95 .

Hypocrateriformis coroll. 52 . 23.

cosandria 24.

Imbricata folia 48. 105.

- - - - calyx.62.

Impari-pinnatum fol. 47.

Imperfectus flos. Riv. 134.

Imperfecti 20. 2 I. 22.

Inæqualis corolla. 223.

Incanum fol. 2 I9.

- - color 244.

Incarnatus color. 244.

Incisum fol. 219.

Incompletx. 25.

- $\quad$ - fios 76.134.

Inconspicuæ 21 .

Incrassati pedunc. 222.233 .

Incumbens 223.

Incurvum fol. 49.

Indivisum fol. 233 .

Inerme fol. 44.233 .

Inflatum pericarp. 223.

Inflexum fol. 49.

Inflorescentia. I I 2.

Infundibuliformis cor. 52.23.

Integer calyx. $\mathrm{h}_{3}$.

Integer caul. 39.

Integerrimum fol. $44 \cdot 233$.

Integrum fol. 44.

Interfoliacei flor. I 12.

Interruptè pinnat. fol. 47 . 
Intorsio habitus. 103.

Involucrum 52 .

Involuta fol. 105 .

Inundata loca. 266.

Inundatx (48. ord. nat.) 32 .

Irregularis fios. $134 \cdot 223.22$.

Isostemones H. 25.

Ju!ifere 20.

Ju! us T. II 3 . I 34 .

Labiatus flos. T. I $34 \cdot 23$.

Lacera coroll. 223.

Lacerum fol. 44 .

Lacinix corolle. 63 .

Laciniatum fol. 43 .

- - - flos. I 34.

Lactescentia. I II.

Lactescentes I 9 .

Lacteus color. 244.

Lacustris pl. 266.

Lamellosi fungi. 27.

Lamina coroll. 52 .

Lana. 109.

Lanatum fol. 45 .

Lanceolatum fol. 42 .

Laterifolii flores. I I 2.

Laxus. 233.

Legumen. 53.

Leguminosæ. I 9. 2 I. 25.

Liber corticis. 37.

Lignosus caul. 233.

Lignum. 37.

Ligulatus flos. 77.

Liliaceæ (1 0. ord. nat.) 23.28 .

Limbo argenteo folia. 2 I 1 .

Limbus. 52.

Lineâ albâ folia. 2 I I.

Linealis. 262.

Lineare fol. 42 .

Linguiforme fol. 46 .

Lingulatus flos. 134.23.

Littora. 265.
Lividus color. 244.

Lobatum folium. 43 .

Loculamentum caps.53.69.

Locusta Raj. I I 3 .

Lo:nentacex ( 56. ord.nat.) 34.

Lunuiatum fol. 43.

Luridx (33. ord. nat.) 3 I.

Luridus color. 244.

Luteus color. 244.

Luxurians flos. 79.95 .

Lyratum fol. 43 .

$1 \sqrt{1}$

flos. 65

Mines agror. 269.

Marina pl. 265.

Maritima idem, ac littoralis. 265.

Mas plinta. 93. 94 .

Masculus fios. 93 .

Medulla pl. 37 .

Mejostemones. 25.

Membranaceum fol. 46 .

Metenrici. 272.

Methodus 97.

Monadelphia 25 .

Monandria 24.

Monangia 20.

Monocoty!edones roz.

- - - petaloidere. 25.

Monocia 25.

MTonoperiantha. 25.

Monoperaloides fios. I 34 .

Monopetalus. 58.22 .

Monophyllus calvx. 62 .

Monophytunthe W. 25.

Monosperma. 70 .

Montes. 267.

Muricatæ (I r ord. nat.) 28.

Multica psulare pericarp. 69.

Multicapsulares. I 9 .

Multiflorus cal. 62. 
Multiloculare pericarp. 69.

Multipartitum fol. 44.95 .

Multiplicatus flos. 80. I 28.

Multisiliqux (23.ord. nat.) 35

$$
37.21 .25 \text {. }
$$

Muscose pl. 19.

Muticus glumac. 233.

Mutilus flos. 79. 128.

N atans fol. 49.

Naturalis character. I 29.

$$
\text { I } 30.13 \text { I. }
$$

Nectarium 53.

Nemora. 267.

Nervosum. fol. $45 \cdot 233$.

Nidulantia semina. $224 \cdot 70$.

Niger color. 244.

Nigro-maculata fol. 2 I I.

Nilidum fol. 45 .

Niveus color. 244.

Nucamentaceæ (I7. ord. nat.) 28.

Nucamentum I 34.

Nudum fol. 233.

- - caul.39.233.

- - flos. 76 .

Nutans flos. 60.

C

bliquitas flor. $\mathrm{IO}_{4}$.

Obliquum fol. 47.

Oblongum fol. 42.233 .

Obtusum fol. 44. 233 .

Obtusum cum acumine fol. 44 .

Obversè ovat. s. cordat. fol. 220.

Obversum folium. 220.

Obvoluta folia. Ios.

Octandria. 24.

Octofidus 63 .

Octcloculare peric. 69.

Oleraceæ (53. ord. nat.) 33 .

Oligantberce 25.
Opposita fol. $4^{8 .}$

Oppositè pinnatum fol. 47 .

Oppositifolii flor. I 12.

- - composit. (2I.d.ord. nat.) 2甲.

Orbiculatum fol. 42 .

Orchidex (4.ord. nat.) 27.

Ordines naturales. 27.

Ordo 1 or . 136.138.

Ordo T. 135 .

Orgya (mensura) 262 .

Ossa. 93.

Ovale fol. 42 .

Ovarium 92. Boerh. I43.

- - fœecundatum. 92.

Ovatum 42.

Ovum vegetabile. 88.86 .92$.

Palatum corolle. 223 .

Palea. 54.

Paleaceus flos Raj. I 34

Palloplastimonopetalce 25 .

Palmaris (mensura) 262.

Palmatum 43 .

Palnie (2.ord. nat.) 27.37.25.

Paludes. 266.

Panduræforme fol. 43 .

Panicula. 4 I

- - coarctata 4I.

- - diffusa. 41.

Papilionacea corolla. 53. 135.

- - (55.ord. nat.) 33. 21.23 .

Papillosum fol. 45 .

Papposæ. 20.

Pappus. 54 .

Parabolicum fol. 42 .

Parallelum Dissep. T. I 35.

Parasiticus caulis. 40.

Partialis umbella. 55 .

Partitum fol. 43 .

Pascua. 268. 
Patens fol. 49.

Patulus peduncul. $22 \mathrm{I}$.

Pedalis mensura. 262.

Pedatum fol. 48.

Pedicellus. 40.

Pedinculus. 40.

Pelta Lichenibus. 223.

Peltatum fol. 49.

Pentafora pericarp. 22.

Pentagonus caul. 40.

Pentandria. 24.

Pentangice. 20.

Pentapetaloides. 58.

Pentapetalus. 58. 22. 64 .

Pentaphyllus calyx. 62.

Perfectus fios. R. I 34.

Perfoliatum fol. 49 .

Perforatæ. (60. ord. nat.) 35 .

Perianthium.52. 57.

Pericarpium. 53. 56.92.

Perpendicularis. 38.

Persistens. 63.65.

Personatæ. (59.ord.nat.) 34 .

Personatus flos. T. I.34.

Petalodes. 58.

Petalum.52. II 3.

Petiolares flores. irir 2.

Petiolatum fol. 49. 233 .

Petiolus. 4I.

Phoeniceus color. 244 .

Piceus color. 244.u

Pileus fungor.223.

Pilosum fol. 45.236 .

Pilus 50.

Pinguis sapor. 285 .

Pinnatifidum fol. 43 ,

Pinnatum fol. 47.

Piperitx. (I. or Re nat:) 127. - - - flos. \$1.82092.

Pistillum. 53..?

\section{- - . T. II3.}

Pistillum abire in fructum. T.
Placenta. V. B. II3. I34.

Placencatio habitus. 102.

Planipetalæ. 2 I.

Planum fol. 46.

Plenusfos. 8 r. 82.83 .84 .85 .

128.195 .

Plicata foliatio. r 06.

- plant.217.

- fol. 45 .

Plumbeus color. 244.

Plumosus pappus. 70.

Plumula. 54.

Pollep 53.56.90.92.

Pollicaris mensura. 262 .

Polyadelphia. 24.

Polyandria. 24.

Polyangice. 20.

Polyanthere. 25.

Polycotyledones. 102.

Polygama planta. 94.95.

Polygamia. 2.5. 40.

Polygonus caul. 40.

Polypetalus. 58.

Polyspermæ. $2 \mathrm{I}$.

Polystemones. 25.

Pomacex: (37. ordinát:) $3 x_{0}$

Pomiferæ. 19: 20. 21.

Pomum. 53.

Porosi fungi. 27.

Præmorsa radix. 38.236.

- - - falou4t

Prasinus color. 244.

Prata. 267. 268.

Precix-(5 E.ord.nat.) 33 :

Prismaticum pericarp. 223.

Procumbens caul. 39. 236 .

Prolifer caulis 4 . 0.

- - frondosús flas. 8r.

Propago. 54.

Proprium receptaculum. 54.

Prunus, 134. 
Pubescens fol. 2 I 9.

Rameum fol. 48.

Pubescentia babitus. 108. 2 15.
Pullus color. $2+$.
Ramificatio.

Pulmones vegetabil. 93 .

Punctatum fol. 45. 211 .

Pungens. 233.

Puniceus color. 244.

Purpureus color. 244.

Putamineæ. (3I. ord. nat.) 3 I.

Q

uadrangulare fol. 43 .

Quadricapsulare pericarp. 69.

Quadrifidum fol. 43 . cal. 63 .

Quadrilobum fol. 43 .

Quadriloculare peric. 69.

Quadripartitum. 44.

Quadrivalve pericarp. 69.

Quadrivasculares 19.

Quaterna fol. 48 .

Quina fol. 48 .

Quinatum fol. 47 .

Quinquangulare fol. 43 .

Ramosa radix. 38 .

Ramosissimus. caul. 40.

Ramosum fol. 48 .

Ramus 36. caulis. 40.233.

Receptaculum 54. 56.

Reclinata folia. I06. 49.

- - caulis. 39.

Regularis corolla 223.22.

Remoti pedunc. 233.

Reniforme fol. 43 .

Repandum fol. 44 .

Repens caul. 39.

- radix. $3^{8}$.

Restantes pedunculi. 222.

Resupinatio florum. 104.

Retusum fol. 44 .

Revolutum folium. 49.

Rhœades. (30. ord. nat.) $3 \mathrm{r}$,

- - - - caulis. 218. Rictus corollae. 223.

Quinquecapsulare pericarp. Ringens corolla. 52.135 .

\section{9.}

Quinquefidum $43 . \operatorname{calyx} .63$.

Quinquelobum. 43.

Quinqueloculare pericarp.69.

Quinquepartitum. 44 .

Quinquevalve pericarp.69.

Quinquevasculares. 19.

\section{Racemus $4 \mathrm{r}$.}

Radiati flores. 72.223.23.

Radicale folium. $4^{8}$.

R - - pedunc. $4 \mathrm{I}$.

Radicans fol. 49 .

Radicatio 102 .

Radicatum fol. 2 I9.

Radicula. $3^{8 .}$

Radius. 23 .

Radix. 38.93 .
Ringentes. 25.

Ripæ. 265.

Rosacea coroll. 23 .

Rostellum seminum. 54.

Rotaceæ. (52. ord. nat.) 33 .

Rotata coroll. 5.2 .

Ro' undum fol. 43 . 433 .

Ruber color. 244.

Rubro-maculata fol. 43.233.

Ruderata loca. 269.

Rugosum fol. 45 .

Rupes. 261.

Sagittatum fol. 43.)

Salsus sapor. 285 .

Sanguineus color. 244.

Sapida. 285.

Sarmentaceæ (49. ord.nat) 32 . 
Sarmentosus caul. 40.

Scaber caul. 40.

Scabridie (20. ord nat.) 29.

Srabrities babitus. 108.

Scabrum folium. 45.

Scanderites Nior. I9.

Scapus. 40.

Scitamina (3. ord. nat.) 27.

Scutellum Lichenib. 222.

Sectio T. 135.

Secunda spica. $4 \mathrm{I}$.

Sedes floris Ray. I34.

Semen. 54. 56.88 .92 .

Semi-amplexicaule fol. 49 .

Semicylindracelim fol. 46 .

Semiflosculosusflos. T. I 34.23 .

- . - (2 r.2.ord. nat.)29.

Semiflosculus, T. 77.

Seminale fol. 48 .

Sena folia 48.

Senticosa. ( 35 ord. nat.) $3 \mathrm{I}$.

Sepiariæ (25. ord. nat.) 30 .

Serratum fol. $44: 233$.

Sesquialtera stanin. 25.

Sesquitertia stamin. 25.

Sessile fol. 49. 233.

Sexangularis caulis. 218.

Sexfidus calyx. 63 .

Sexloculare pericarp. 69.

Sexus. 86. seq.

Siccus sapor. 285 .

Siliqua. 53.

Siliquosa (57. ord. nat.) 37. I9. 2 I. 25 .

Simplex caulis. 39.

- - flos 76 .

- - folium. 42.233.

- - radix. $3^{8}$.

Simplex umbella. 54.78 .

Simplicissimus caul. 233.

Sinistrorsùm volubilis.39. 103.

Sinuatum folium. 43.
Solidus bulbus. 50.

Solitarius pedunc. $4 \mathrm{I}$.

Solum plantarum. 265.

Spadiceus fios. 77.

Spadix. 55.

Sparsa folia. 48 .

- pedunculi. 4r.

Spatha. 52.

Spathacex (8.ord. nat.) 28.

25.

Spathulatum fol. 42.

Species 99. I oI.

Specifica nomina. 202.

Spica. 4 I.

- disticha. 4I.

- - secunda. $4 \mathrm{r}$.

Spicula graminit ${ }^{\circ} 2,23$.

Spina 5o. r ro.

Spinosum fol. 44.

Spithamalis mensura. 262.

Squamosus bulbus. 50.

- - culmus 40.

Squarrosus calyx. 62.

Stagna 266.

Stamen. 53 .

Stamineusflos. I 34. 19.20.21.

Statuminatæ (6r. ord. nat.) 35 .

Stellata fol. 48 .

Stellatæ (44.ord.n.) 32.2 I. I9.

- - flos. Mor. 134.

Sterilis flos. T. I 34 .

Stigma. 53. 56.91.92.

Stimuli I09.

Stipes. 42.

- - pappi semin. 54.

Stipticus sapor. 285 .

Stipulit. 50.

Stipulatio. I08.

Striatum fol. 45.

- caulis. 40.

Strictus. 2 19. 233.

Striga. 109. 
Strigosum fol. 2 19. 233 .

Strobilus. 54.

Stylus. 53. 56.92.

Subalare fol. 48 .

Subdivisus caulis. 40.

Submarinæ herb. 20. 2 I

Subrotundum fol. 42.223 .

Subulatum fol. 43 .

Succulentæ. (46. ord.nat.) 32 .

Suffrutices. I 8.

Sulcatum fol. 46 .

- - caulis. 40.

Sulphureus color. 244 .

Supra decompositum fol. $4^{8}$.

Sylvæ. 267.

Syngenesia. 25 .

Synopsis. 98.

Synoptica dispositio. 97.

Systema. 98.

Systematica dispositio. 97.

\section{7}

emperatæ plantæ. 277.

Tenuifolia plant. 242.

Teres folium. $45 \cdot 233$.

Terminalis peduncul. $4 \mathrm{I}$.

Terna fol. 4.8. 222.

Ternatum fol. 47.

Terrestras pl. imperfect. 20.

Tesselata fol. 2 I I.

Testaceus color. 244.

Testes. Testiculi vegetab. 92.

Tetra vegetab. odore. 285 .

Tetrafora peric. Camell. 22.

Tetragonus caulis. 40.

Tetramacrostemones. 25.

Tetrandria. 24 :

Tetrangice. 20.

Tetrapetalus 58.22 .

Tetraphyllus calyx. 62 .

Tetrasperma 70.

Thalamus. 92.134.

Thyrsus. 4I.
Tomentosum fol. $45 \cdot 233$.

Tomentum. rog.

Torosum fericarp. 224.

Tortilis arista. 223 .

Trachex. 37.

Triandria. 24.

Triangia 20.

Triangulare fol. 42 .

Triangularis caul. 40 .

Tricapsulare pericarp. 69. I9.

Tricoccæ (47. ord. nat.) 32 . 25. I 9 .

Trifidum fol. $43 \cdot$ cial. 63 . Triflorus pedunc. 4I.

Trifora pericarpia. 22.

Trigonus caulis. 40.

Trihilatæ (50. ord. nat.) 33 .

Trilobum fol. 43 .

Triloculare pericarp. 69.

Tripartitum fol. 44 .

Tripetalodeæ (6. ord. nat.) 28.

Tripetalus flos. 58.22 .

Triphyllus calvx. 62 .

Tripinnatum fol. 48 .

Triplicato-pinnatum fol. 48 .

Triplicato-ternatum fol. 48 .

Triplicatus fios. 80.

Triqueter cailis. 40 .

- - - scapus.218.

- - - folium. 43.46.

Trisperma 70.

Triternatum fol. 48 .

Trivalve pericarp. 69.

Trivasculares. 19.

Trivialia nomina. 202.

Tropici solares flores. 273.

Truncatum fol. 44.

Truncus. 39.93 .

Tuba. V. II 3 .

Tuberculum lichenib. 222 .

Tuberosa Rad. 38. 


\section{TER M I N I.}

Tubulosi f.osculi. $\not$.

…... fol. 46.233 .

Tubus 52:

Tunicatus bulbus. 50.

Turbinatum pericarp. 224.

Uliginosa loca. 266.

Un bella 54.72 .

Un. bellatie (22. ord. nat.) 30. $23.24 \cdot 25$.

A. fl. 76.78 .

Úmbelliferx. 19. $2 \mathrm{I}$.

Umbellula..79.

Uimbilicatum fol. 219.

Unbilicatus fructus. 19. 20.

Umbo Morison. I 34 .

Uncialis mensura. 262.

Undulatum fol. 45.

-....pedunc. 282 .

Unguicularis mensura. 262. Unguis 52.T. II 3. .

Unicapsularis 68.

Uniflorus 4I.

Uniformis. 22.

Uniloculare pericarp. 69.

Univalve pericarp. 69.

Univasculares. 19 .

Universalis umbella. 55 .

Urceolata corolla. 223.

Utriculi 37. I I.I.

V

agx (ord. ngtur.) I 25.

Vagina uteri. 92.

Vaginales (27. ord, nat.) 30 .
Vagináns fol. 49.

Valvuila capsulde. 53.69.

Variegata fol.: 236 . ju 2 .

Varietas 100. I0I.

Vasa chylifera.pl. 93.

.... succosa. 37.

..... spermatica 92.

Venosum fol. 45.

Ventriculus 93.

Vepreculæ (54. ord. nat.) 37 .

Vernales 276..

Versatiles Anthera. 223.5 J/2

Versuræ 269.

Verticalia fol. 220.

Verticaliter ovata, s, cordata, 220.

Verticillata folia. 49 .

Verticillatæ (58.ord. nat.) 34 .

21. 19.

Verticillus flo 48.

Vexillum papilionac. I 35 . 1 A

Vigendi tempus 276 .

Vigilix plant. 272.

Villosus caul. 40.

Violaceus color. 244.

Viridis color..244.

Viscidus. 45. .7I

Viscosus sapor. 285.

Vita vegetabilium. $\$ 8$.

Vitreus color. 243.

Volubilis caulis. 39.233.

Volva 52.

Vulgaris planta. 216.

Vulva. 92. 


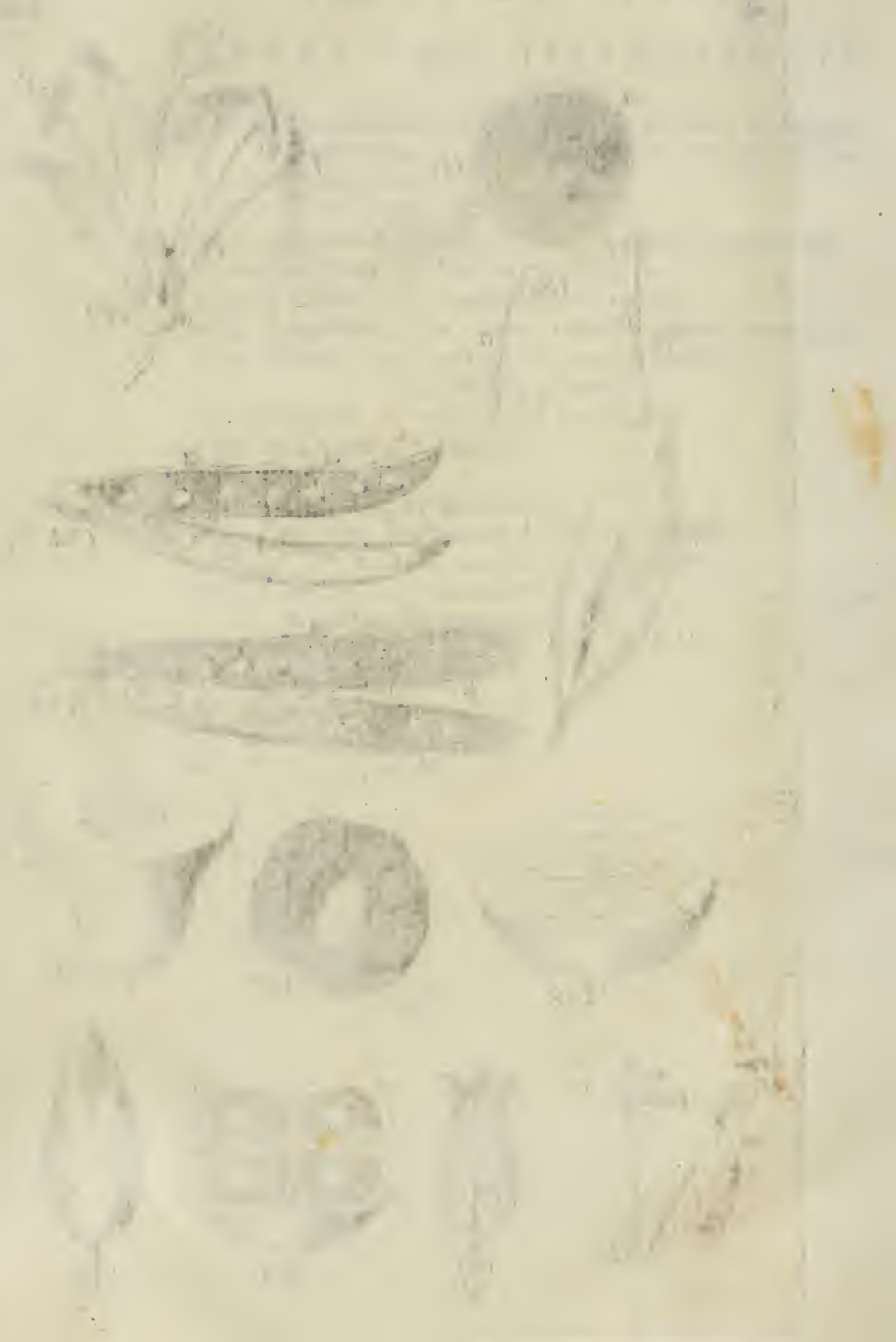




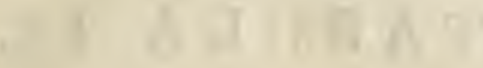

autuokugare

$$
\begin{aligned}
& \text { x- } \\
& \text { if } \sec 20.8 \text {. }
\end{aligned}
$$

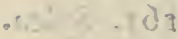

$$
\therefore \text { aid. } 7=17.02
$$

$$
-x=1+2-1, y
$$
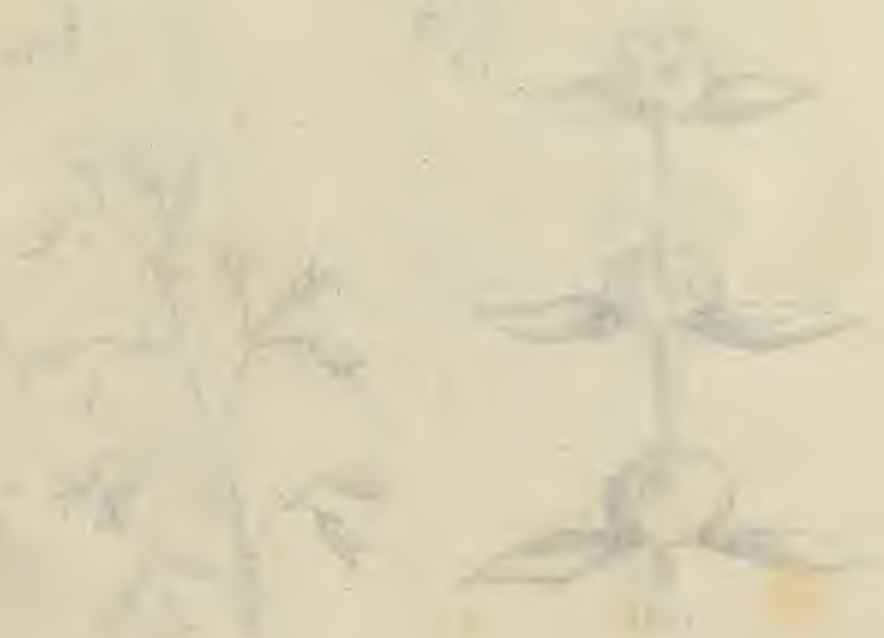

$+$

Clit. 


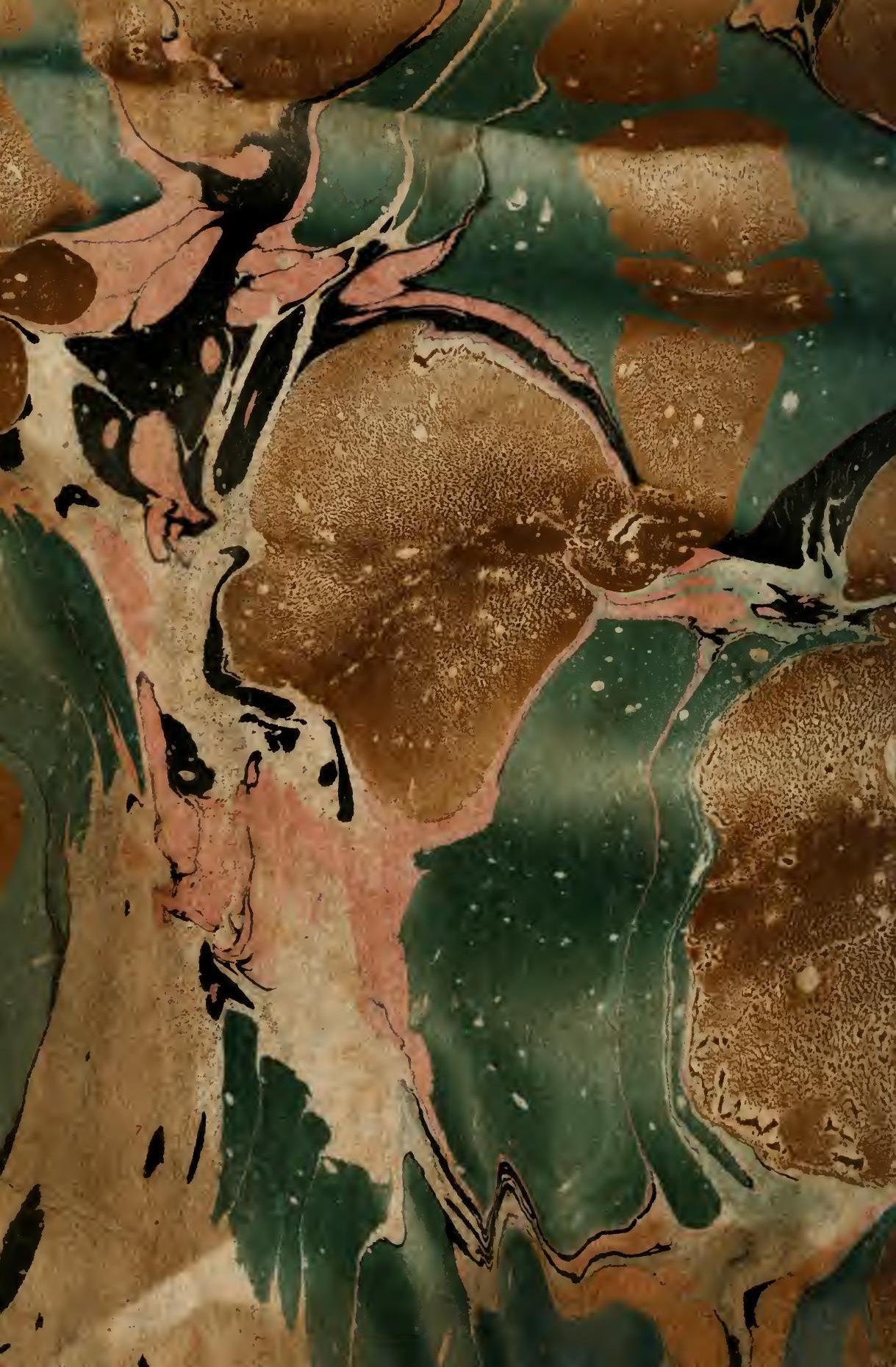




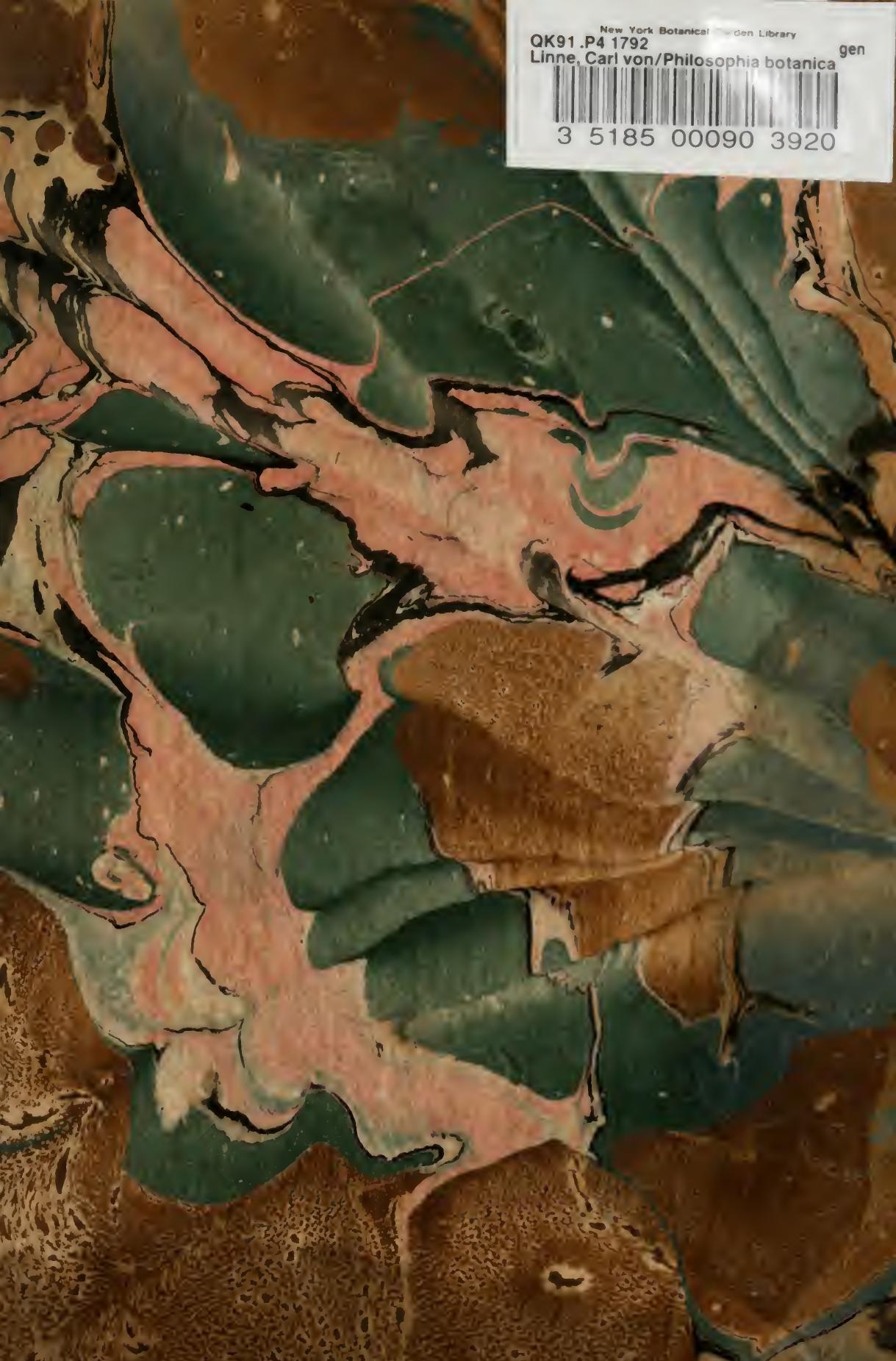




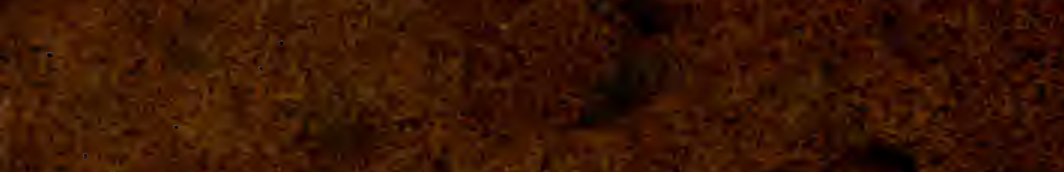

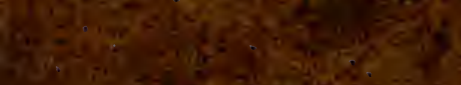

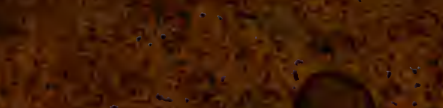

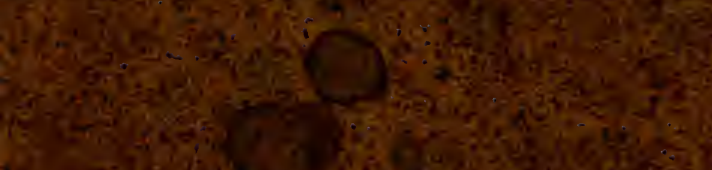

and

Chis

Is

mon

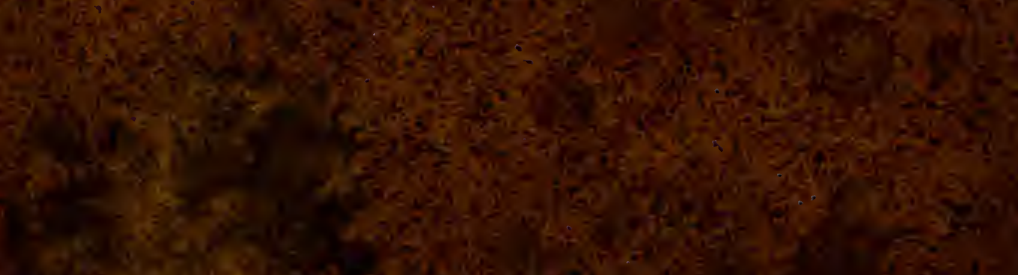

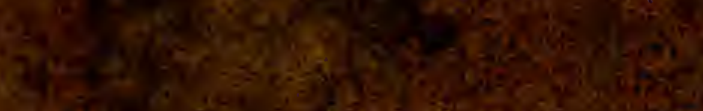

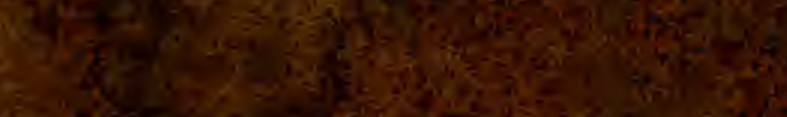

tis

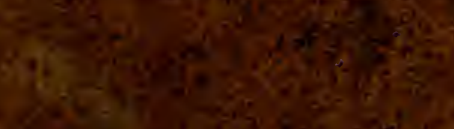

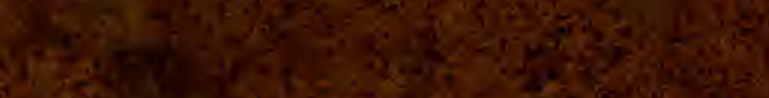

35.

87.

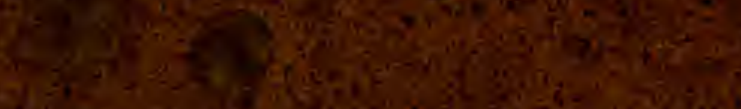

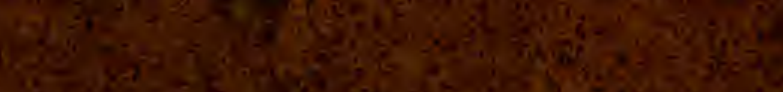

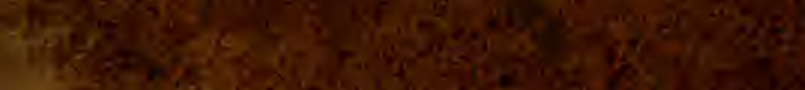

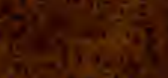

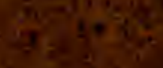

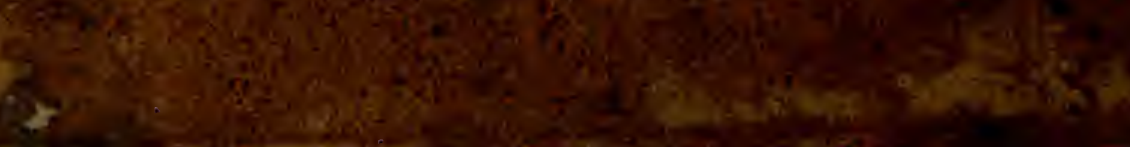

